INDEX OF

\title{
BRITISH PLANTS
}

ROBERT TURNBULL 


$$
16
$$




\title{
INDEX OF BRITISH PLANTS
}

ACCORDING TO THE

\section{LONDON CATALOGUE}

\author{
(EIGHTH EDITION) 1886.
}

INCLODING

THE SYNONYMS USED BY THE PRINCIPAL AUTHORS, AN ALPHABETICAL LIST OF ENGLISH NAMES, ALSO REFERENCES TO THE ILLUSTRATIONS OF SYME'S ENGLISH BOTANY, AND BENTHAM'S BRITISH FLORA. :

BY ROBERT TURNBULL.

LONDON :

GEORGE BELL AND SONS, YORK STREET, COVFNT GARDEN. 


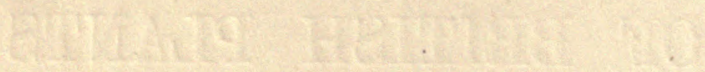

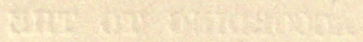

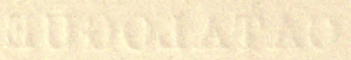

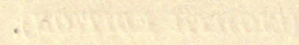

6hinathanger

?. 


\section{PREFACE.}

\section{THE purpose of these pages is to furuish a complete Index of British Flowering Plants and Ferns.}

The work is based upon the London Catalogue, Part I., 8th Edition, and contains all the names there found, together with all the additional names of species under which descriptions are given in ten of the best known English botanical works, viz.: English Botany; Bentham's Handbook; Babington's Manual; Hooker's Student's Flura; British Wild Flowers; Lindley's Synopsis; Hooker and Arnott's British Flora; Withering's Arrangement; Notcutt's Handbook; Hayward's Pocket-Book.

The corresponding name in the London Catalogue is indicated in most cases in the text by the sign of equality, while the foot-notes give for each name from the London Catalogue the synonyms used in the works above mentioned.

The compiler, though conscions that the result of his attempt is not all that he conld desire, ventures to hope that it may in some degree lessen the perplexities of nomenclature to the botanist.

In the side colnmns are given references to the numbers in the London Catalogue, the figures in English Botany, 3rd Edition, and Fitch and Smith's Tllustrations of Bentham's British Flora. A name in brackets is one which has been suggested, from the most recent resnlts of the stady of authorities, as preferable to the accompanying name from the London Catalogue. The census number, where it has been recorded, is added in larger type to each plant, also the time of flowering, \&c.

Names in italics (indicating aliens) and the names of authorities in brackets (indicating that these authorities regarded the plant as a species) stand as they appear in the London Catalogue.

Special attention has been given to the accentuation, which has been based, as far as possible, on the deriration of the words (where this is known), and as a further aid to pronunciation the quantity of a syllable has occasionally been marked.

The compiler desires to record his sincere thanks to many friends for valuable suggestions and advice, especially to Mr. B. Daydon Jackson and the late Rev. J. H. Thompson. 


\section{ADDITIONS AND CORRECTIUNS.}

Page 2, line 11, for Lam. read Scop.

P. 2, 1. 22, for [alba, L.] read See alba, L.

P. 2, 1. 54, for [conjuncta, Bab.] read See conjuncta, Bab.

P. 2, 1. 55, for Lam. read Scop.

P. 4, l. 57, after margaritacea, R.Br. insert [Anaphalis margaritacea, Benth. et Hook. f.].

P. 5, 1. 11, for Tri. read Dian.

P. 5, 1. 17, for Pers. read Bernb.

P. 6, 1. 7, for Hook. read J. Gay.

P. 11, after 1. 54, insert ramosus, Huds. See asper, Murr.

P. 15, 1. 4, for 1826 read 1626 .

P. 16, after 1. 42, insert verna, Chaix. See præcox, Jacq.

P. 17, Il. 3 and 4 , after Benth. insert et Hook. f.

P. $18,1.9$, for $\mathrm{i} .218$ read ii. 218 .

P. 18, 1. 24, for $1206,7 \mathrm{read} 1276,7$.

P. 18, 1. 44, for 1909 read 1919.

P. 22, 11. 17-19, for Pol'ygōna'tnm read Pol'ygon'ătum.

P. 22, 1. 48, for Colnut read Cobnut.

P. 24, 1. 41, for 968 read 969 .

P. 28, 1. 44, for $E$. angustifolium, Roth. read E. angustifolium, Roth.

P. 37, after l. 44, insert alpina, R.Br.

P. $38,1.47$, for 117 read 1170 .

P. $44,1.26$, for 1251 read 1521 .

P. 46, 1. 2, for 711 read 1111 .

P. $46,1.36$, for 278 read 277 .

P. $53,11.37$ and 38 , for 8.25 read 865 .

P. 55, 1. 31, for Hawkweed read Hawkweed Ox-tongue.

P. 55, 1. 49, for Chester read Cluster.

P. 56, foot-note 1, for East read Earth.

P. $60,1.41$, for vii.1122 read xi.1722.

P. 62, 1. 3, for 22 read 21 .

P. 63, 1. 25, for Rhyncospora read Rhynchospora.

P. 73, after 1. 25 insert elegans, Mack, $=$ S. Geum, v. elegans.

P. $78,1.2$, for $139 \mathrm{~b}$ read 1396 .

P. $78,1.48$, for iv. read vi. 


\section{INDEX OF BRITISH PLANTS.}

L.C. denotes the London Catalogue of British Plants, 8th ed.

E. B. " Sowerby's English Botany, 3rd ed, ed. Boswell (Syme).

B. " Fitch and Smith's Illustrations of Bentham's British Flora.

The censns numbers are taken from the L.C. a. denotes annnal, b. biennial, p. perennial, s. shrub, t. tree. The numbers after these letters refer to the months when the species flower in Britain. $A$ reference in parentheses denotes a description of the plant under a synonym.

Ac'er, L. SA'PINDA'Cex, Soapworts. Oct. Mon. campest're, L. 62. t. 5, 6. Small-leaved Maple. Common $M$. v. he'becarp'um, DC.

Pseud'o-plat'anus, L. t. 5, 6. Sycamore. Greater Maple.1

Ac'eras, R. Br. Orchí'ex, Orehids. Gyn. Mon. an'thropoph'ora, ${ }^{2}$ R. Br. 17. p. 6, 7. Green Man Orchis. ${ }^{3}$

Achille'a, L. Compos'ix s, Composites. Syn. Sup. de'colo'rans," Schrad. p. 8. Serrated or Dotted-leaved Yarrow. Millefol'ium, L. 112. p. 5-9. Common Yarrow. Milfoil. Ptarm'ica, L. 108. p. 7-9. Sneeze-wort Yarrow. Gosse-wort. ${ }^{\text {s }}$ serra'ta, Sm. = A. de'colo'rans, Schrad. tan'ace'tifol'ia, All. p. 7. Tansy-leaved Yarrow. to'mento'sa, L. p. 7,8. Woolly Yellow Yarrow. Golden Milfoil. Achnod'on, Trin. ar'ena'rius, Trin. = Phle'um ar'ēnárium, L.

Ac'inos, Moench.

vulga'ris, Pers. = Cal'aminth'a arvens'is, Lam.

Aconi'tum, L. Raxunc'ula'cese, Crowfoots. Polyan. Pol. Napell'ns, ${ }^{6}$ L. 7. p. 7-9. Monkshood. Wolfsbane. Aconite. vulga're, DC. $=\mathbf{A}$. Napell'us, L.

319

$319 \mathrm{~b}$

318

1357

ix. 1447

จ.729

จ.727

จ.730

จ.728

จ. 726

B.

219

220

1006

530

529

ix. 1391

942 Cal'amus, L. 31. p. 6, 7. Sweet-flag. Sweet Sedge. Myrtle-flag.

Actæ'a, L. Ranunc'dua'Cex, Crowfoots. Polyan. Mon. spica'ta, L. 5. p. 5. Herb Christopher. Baneberry.

ix. 1442 Dam'aso'nium, Hook. = Dam'aso'nium stella'tum, Pers.

Ad'iant'um, L. Fir'ices, Ferns. Crypt. Fil. Capill'us-Ven'eris, L. 8. p. 5-9. True Maiden-hair.

Ado'nis, L. Raxunc'ula'ces, Crowfoots. Polyan. Pol. autumna'lis, L. a. 5-9. Autumnal or Corn Pheasant's-eye.

Adox'a, L. CAP'rifol'in'CEx, Caprifoils. Oct. Tet. Mos'chatellína, L. 88. p. 4, 5. Tuberous Moschatel.

Agopod'ium, L. Umbelli'́ters, Umbellifers. Pent. Dig. Pod'agra'ria, L. 100. Gout-weed. Bishop's-weed. Goat-weed. Æthu'sa, L. UMBellif'ERx, Umbellifers. Pent. Dig. Cy̆nap'ium, L. 91. a. 7, 8. Fool's Parsley.

Agraph'is, Link. nu'tans, Link = Scill'a nu'tans, Sm.

Agrimo'nia, Tourn. Rosı'ces, Roseworts. Dod. Dig. Eupatória, L. 98. p. 6-8. Common Agrimony. "dora'ta, Mill. 33. p. 7. Fragrant Agrimony.

A'gropy'ron, Gærtn. Grïuñ'ex, Grasses, Tri. Dig. acu'tum, ${ }^{\prime}$ Gren.\& Godr. 25. p. 8, 9. Decumbent Sea Conch-grass. ${ }^{9}$ cani'num, ${ }^{10}$ Beauv. 82, p. 7. Wood Conch-grass. Dog's Wheat. ${ }^{11}$ v. b̌flo'rum, Mitt.

1 Scotch Plane. 2 Ophrys anthropophors. 3 Man Tway-blade. 4 Achilles serrata. 5 Goosetongue. 6 Aconitnm vulgare. 7 Herb Gerarde. 8 Triticnm seutum. T. laxnm. 9 Flat-stemmed Sea Wheatgrass. 10 Triticum caninnm. 11 Fibrous-rooted or Bearded Wheat-grass.

\section{4 xii. 1887 \\ i. 13 \\ ir. 636 \\ iv. 580 \\ iv. 600 \\ 424 \\ iii. 417 \\ iii.418 \\ 1752 (xi. 1812) \\ 1749 (xi. 1809) \\ 1206}


A'gropy'ron, Grrtn.-continued.

crista'tum,' Schreb. p. 7. Crested Wheat-grass.

junc'eum, ${ }^{2}$ Beauv. 36. p. 7, 8. Sand C. Bushy Sea Wheat-grass. pung'ens, ${ }^{3}$ R. \& S. 16. p. 8. Erect Sea Couch-grass.

v. littora'le (Reichb.)

v. pycuanth'um (Gren. \& Godr.)

re'pens, ${ }^{4}$ Bealtr. 108. p. 6-8. Creeping $W$. or $C$. Dog-grass.

v. barba'ta, Duval-Jouve.

v. obtu'sa, Syme.

Agrostemm'a, L.

Githa'go, L. = Lych'nis Githa'go, Lam.

Agro'stis, L. Grimin'Es, Grasses. Tri. Dig.

alb'a, L. [palust'ris, Huds.] 103. p. 7-9. Marsh Bent-grass.

v. stolonif'era, ${ }^{5}$ (L.) Fiorin-grass.

v. marit'ima, Mey.

an'emagro'stis, Syme.

austra'lis, L. = Gastrid'ium lendig'erum, Gaud.

cani'na, ' L. 93. p. 7, 8. Brown Bent-grass.

interrupt'a, L. = Ape'ra interrupt'a, Beauv.

littora'lis, Sm. = Polypo'gōn littora'lis, Sm.

ni'gra, With. = A. vulga'ris, v. ni'gra.

palust'ris, Huds. [alb'a, L.]

panic'ea, Ait. = Polypo'gōn mons'peliens'is, Desf.

seta'cea, ${ }^{7}$ Curt. 12. p. 6,7. Bristle-leaved Bent-grass.

Spi'ca-vent'i, L. = Ape'ra Spi'ca-vent'i, Beauv.

stol'onif'era, Sm. = A. alb'a, v. stol'onif'era.

vulga'ris, With. 112. p. 6-9. Common or Fine Bent-grass.

v. pu'mila (L.)

v. ni'gra $^{8}$ (With.)

Ai'ra, L. Grämix'ex, Grasses. Tri. Dig.

alpi'na, L. = Deschamps'ia alpi'na, R. and S.

aqua'tica, L. $=$ Cătabro'sa aqua'tica, Beauv.

cæespito'sa, L. = Deschamps'ia caspito'sa, Beauv.

canesc'ens, $\mathbf{L} .=$ Coryne'phorus canesc'ens, Beaur.

car'yophyll'ea, L. 105. a. 6, 7. Silvery Hair-grass.

v. aggrega'ta, Tim.

crista'ta, L. = Koele'ria crista'ta, Pers.

flexuo'sa, L. = Deschamps'ia flexuo'sa, Trin.

laviga'ta, Sm. = Deschamps'ia alpi'na, Beauv.

ma'jor, Syme.

præ'cox, L. 108. a. 4, 5. Early Hair-grass.

seta'cea, Huds. = Deschamps'ia seta'cea, Hanb.

uli'gino'sa, Weihe $=$ Deschamps'ia seta'cea, Hanb.

Airochloa, Link.

crista'ta, Link $=$ Koele'ria crista'ta, Pers.

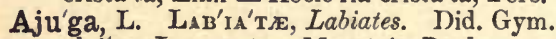

alpi'na, L. p. 6, 7 . Mountain Bugle.

Chamæ'pity̆s, Schreb. 8. a. 5-9. Ground Pine. Yellow Bugle. genevens'is, L. var. Beuth. = A. py'ramida'lis, L.

py'ramida'lis, ${ }^{\circ}$ L. 11. p. 5-7. Pyramidal Bugle.

rept'ans, L. 103. p. 5-7. Common Bugle.

Alchemill'a, Tourn. Rosa'ces, Roseworts. Tetr. Mon.

alpi'na, L. 27. p. 6-8. Alpine Lady's-Mantle.

argent'ea, Din. [conjunct' $\mathrm{a}, \mathrm{Bab}$.]

arvens'is, Lam. 108. Parsley Piert. Field Lady's-Mantle.

conjunct'a, Bab. [argent'ea, Don.] 3. p. 7, 8. Silvery or Silky Lady's-Mantle.

vulga'ris, L. 101. p. 6-8. Common Lady's-Mantle.

v. monta'na, "Willd."

2 T. junceum.

3 T. pungens.

\begin{tabular}{l|c} 
L.C. & E.B. \\
1753 & $($ xi. 1813$)$ \\
1751 & $(\mathrm{xi} .1811)$ \\
$175 \mathrm{lb}$ & \\
$1751 \mathrm{c}$ & \\
1750 & $(\mathrm{xi} .1810)$ \\
$1750 \mathrm{~b}$ & \\
$1750 \mathrm{c}$ &
\end{tabular}

B.

B.

1205

1656

xi. 1719

1176

$1656 \mathrm{~b}$

xi. 1720

$1656 \mathrm{c}$

xi. 1715,6

1655

xi. 1718

1177

xi. 1716

1656

1654

xi. 1717

1178

xi.1715 1179

1657

xi. 1721

$1657 \mathrm{~b}$

$1657 \mathrm{c}$

xi.1731

xi. 1730

1155

$118 \%$

1670

xi.1734

1189

$1670 \mathrm{~b}$

xi. 1732

1186

xi. 1730,1

1671

xi.1735

1188

xi. 1733

1 Triticum cristatum.

- Trichodinm caninum. 7 T. setacea. 8 Agrortis nigra. 8 Ajuga genevensis.

$4 \mathrm{~T}$. repen
Ajuga genevensis.

\begin{tabular}{|r|r}
1162 & vii.1090 \\
1161 & vii. 1089 \\
1160 & vii. 1088 \\
499 & iii. 425 \\
500 & \\
497 & iii. 422 \\
500 & iii. 424 \\
498 & iii. 423 \\
$495 b$ &
\end{tabular}

818

817

(817)

816

322

323

321

5 Agrostis stolonifera. 
Alis'ma, L. Alismácex, Alismads. Hex. Pol.

Damaso'nium, $\mathrm{L}$. = Damaso'nium stella'tum, Pers.

na'tans, L. = Elis'ma na'tans, Buchen.

Planta'go, L. 99. p. 6-8. Greater Water-Plantain.

v. lanceola'tum (With.)

ranunc'uloï'des, L. 84. p. 5-9. Lesser Water-Plantain.

v. re'pens ${ }^{1}$ (Davies) Creeping W. $P$.

re'pens, Davies $=A$. ranunc'uloï'des, v, re'pens.

Alissu'ce.e, R. Br. Alismads.

Alliária, Scop.

offic'îu'lis, Andrz. = Sisymb'rium Allia'ria, Scop.

All'ium, L. Li'Lia'Ces, Lilyworts. Hex. Mon.

ambig'uum, Sm. p. 6. Rose-coloured Garlic.

Amp'elopras'um, L. p. 7, 8. Wild Leek. ${ }^{2}$

v. genui'num, Syme.

v. bulbif'erum, Syme.

v. Babingto'nii s (Borr.) Babington's Leek.

ar'êna'rium, L. $=$ A. Scor'odopras'um, L.

Babingto'nii, Borr.=A. Amp'elopras'um, v. Babingto'nii.

carina'tum, L. 3. p. 8. Mountain G. Purple Field G. Keeled G. ol'era'ceum, L. 47, p. 7. Field or Wild Garlic.

v. complana'tum (Borr.)

paradox'um, Don. p. 4.

Scho'nopras'um, L. 7. p. 6,7. Garden Chives. Chive Garlic.

Scor'odopras'um, ${ }^{4}$. 16. p. 5-8. Sand Leek or Garlic.

sibir'icum, L. 1. p. 6, 7. Greater Chives. Cornish C.

sphæroceph'alum, L. 1.p. 6-8. Small Round-headed Garlic.

trĭque'trum, L. 1. p. 5,6. Guernsey Ramsons. Trian.-stalked G. ursínum, L. 99. p. 4-6. Ramsons. Broad-leaved $G$.

vi'nea'le, L. 76. p. 6, 7. Crow Garlic.

v. capsulif'erum, Syme.

v. bulbif'erum, Syme.

v. compact'um (Thuill.)

Alloso'rus, Bernh.

crisp'us, Bernh. = Cryptogramm'a crisp'a, R. Br.

Aln'us, Tourn. Cu'PULIF'ERE. Monœc. Tet. glu'tino'sa, ${ }^{5}$ Grertn. 109, t. 3, 4. Alder.

Alo'pecu'rus, L. Granin'Ex, Grasses. Tri. Dig. agrest'is, L. 60. a. 5-10. Slender Fox-tail-grass. Black Bent. alpi'nus, Sm. 6. p. 7,8. Alpine Fox-tail-grass.

v. Watso'ni, Syme.

bulbo'sus, Huds. 22. p. 5-7. Tuberous Fox-tail-grass.

fulv'us, Sm. 25. p. 6-9. Orange or Spike-anthered F.

gen'icula'tus, L. 108.p. 5-8. Bent-stemmed, Floating or Marsh F.

v. prónus, Mitt.

palust'ris, Syme.

pratens'is, L. 104. p. 5, 6. Meadow Fox-tail-grass.

Alsi'ne, Wahl.

Cherle'ria, Fenzl. = Ar'ēna'ria sed'oi'des, Schultz.

fasti'gia'ta, ${ }^{6}$ Bab. a. 6. Level-topped Sandwort.

mari'na, Sm. = Lĕpigo'num margina'tum, Koch.

rubell'a, Wahl.=Ar'ēna'ria sulca'ta, Schlecht.

ru'bra, Wahl. = Lěpigo'num ru'brum, Fr.

strict'a, Wahl. = Ar'ềna'ria üli'gino'sa, Schleich.

ten'uifol'ia, Crantz.=Ar'êna'ria ten'uifol'ia, L.

üli'gino'sa, Vill. = Ar'ēna'ria üli'gino'sa, Schleich.

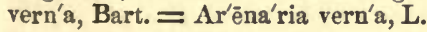

L.C.

E.B.

B.

ix.144I

968

1470

ix. 1437

966

$1470 \mathrm{~b}$ ix. 1438

1471 ix. 1439

$1471 \mathrm{~b}$ ix. 1440

1470

ix. 1433

1397

1397 a

$1397 \mathrm{~b}$

$1397 \mathrm{c}$

ix. 1530

ix. 1531

1402

1401

$1401 \mathrm{~b}$

1406

1403

1398

1404

1399

1405

1407

1400

$1400 \mathrm{a}$

$1400 \mathrm{~b}$

$1400 \mathrm{c}$

ix. 1535

ix. 1536

ix. 1537

ix. 1532

ix. 1538

ix. 1533

ix. 1539

ix. 1540

ix. 1534

1045

1046

1044

1047

1050

1049

1048

1276

1274

viii. 1294

908

xi. 1699

1168

$16+2$

1647

$1647 \mathrm{~b}$

1645

1643

1644

$1644 \mathrm{~b}$

1646

xi.1704

1171

xi. 1702

xi. 1700

xi.1701

1170

xi.1700-2

xi. 1703

1169

ii. 240

ii. $243 b$ is

ii. 242

ii. 243

ii. 244

ii. 241 
Althæ'a, L. Malva'ces, Mallowworts. Monad. Pol. hirsu'ta, L. 2, a. 8,9. Hispid Mallow. Rough-leaved Marsh-M. offic'ina'lis, L. 28. p. 7, 8. Marsh-Mallow. Guimauve.

Alyss'um, L. CruciF ers, Crucifers. Tetrad. Silic. calyci'num, L, a. 6-8. Calyeine Alyssum. Large-calyxed Mad-wort. inca'num, $\mathbf{L}$.

marit'imum, ${ }^{1}$ L. a. or p. 8, 9. Swect Alyssum. Sea-side A. sati'vum, Sm. = Came'lina sati'va, Crantz.

Am'ARANTA'CEs, Amaranths.

Amaranth'us, L. Am Aranta'ces, Amaranths. Monoc. Pent. Blit'um, L. a. 8. Wild Amaranth. Wild Blite. re'troflex'us, L. a. 8,9 .

AM'ARYLLID'Es, R. Br. Amaryllids.

A'Mentifiers.

Amm'i, Tourn. Umbellif'ere, Umbellifers. Pent. Dig. majus, L. Common Bishop's-weed.

Ammoph'ila, Host. GRĀMr'Es, Grasses. Tri. Dig. ar'êna'ria, Host. = A. arund'ina'cea, Host. arund'ina'cea, ${ }^{2}$ Host. 60.p. 7. Marram. Sea Bent, Reed or Matbalt'ica, ${ }^{8}$ Link. 2. p. 8, 9. Baltic Marram.

An'acampt'is, Rich. py'ramida'lis, Rich. = Orch'is py'ramida'lis, L.

An'achar'is, Rich.

alsi'nas'trum, Bab. = Elo'dea canadens'is, Mich.

Anacy'clus, L. radia'tus, Lois. I. a. 7.

Ånagall'is, Tourn. Pri'Mula'ces, Primworts. Pent. Mon. arvens'is, L. 96. a.5-10. Scarlet Pimpernel. Poor Man'sWeatherglass. ${ }^{4}$

v. phœni'cea, Syme.

v. cærul'ea, Bab. = A. cærul'ea, Schreb. cærul'ea, ${ }^{5}$ Schreb. 48, a. 5-10. Blue Pimpernel. tenell'a, L. 93. p. 7, 8. Bog Pimpernel.

Anchu'sa, L. Borấgin'ex, Borageworts. Pent. Mon. arvens'is, Bieb. = Lycops'is arvens' is, $\mathrm{L}$. offic'ina'lis, L. b. 6,7. Common Alkanet. semp'ervir'ens, $\mathrm{C}$. p. 5, 6. Evergreen Alkanet.

Androm'eda, L. ER'1́A'CEe, Heathworts. Dec. Mon. Polifol'ia, L. 29. s. 5.8. Marsh Cistus. Wild Rosemary.

Androsæ'mum, All. offic'îna'le, All. = Hyper'icum Androsæ'mum, L.

An'emagro'stis, Trin. Spi'ca-vent'i,'Trin. = Ape'ra Spi'ca-vent'i, Beauv.

Anemo'ne, L. Ranunc'ula'cex, Crowfoots. Polyan. Pol. a'pennina, L. p. 4. Blue Mountain Anemone. nem'oro'sa, L. 105. p. 4, 5. Wood Anemone. Wind-flower. Pulsatill'a, L. 18. p. 5, 6. Pasque-flower. ranunc'uloi'des, L. p. 5, 6. Yellow Wood Anemone.

Ane'thum, Tourn.

Fœnic'ulum, L. = Fœnic'ulum offic'îna'le, All.

Angel'ica, L. UMBellif'ERx, Umbellifers. Pent. Dig. Archangel'ica, L. = Archangel'ica offic'ina'lis, Hoffm. sylvest'ris, L. 110. p. 8. Wild Angelica.

Antenna'ria, Br. Coupos'rxe, Compositcs. Syn. Sup. dioic'a, ${ }^{6}$ R. Br. 84. p. 6, 7. Mountain Evcrlasting. v. hyp'erbor'ea ${ }^{7}$ (Don.) margar ${ }^{\prime}{ }^{\prime} a^{\prime} c c a,{ }^{8}$ R. Br. p. 8. Pearly Everlasting, Amer. Cudweed.

1178

1380

628

1667

1668

(xi.1722)

956

vii. 1146,7

vii. 1146

vii. 1147

957

(vii.1147)

958

vii. 1148

658

vii.1111

988

vii. 1112

989

vii. 1113

917

vi. 883

701

702

630

1 Glyce maritima,-Koniga maritims. 2 Ammophila arenaria.-Arundo arenaria.-Psamma arenaria. 3 Psamma baltica. 4 Shepherd's Weather-glass. 5 Anagallis arvensis, v. cærulea. 6 Gnaphalium dioicum. 7 G. dioicum, v. hyperborea. $8 \mathrm{G}$. margaritaceum. 
Anth'emis, L. Compos'rx an'glica, $\mathrm{Spr} .=\mathrm{A}$. arvens'is, $\mathbf{v}$. an'glica.

arvens'is, L. 69. a. 7-10. Corn Chamomile.

v. an'glica ${ }^{1}$ (Spr.) Sea Chamomile.

Cot'ula, ${ }^{2}$ L. 72. a. 6.9. Stinking Mayweed. Fetid $C$.

marit'ima, $\mathrm{Sm}_{.}=\mathbf{A}$. arvens'is, v. an'glica.

no'bilis, L. 46. p. 7-9. Common Chamomile.

tincto'ria, L. p. 7, 8. Yellow C. Ox-eye C.

Anther'icum, L.

serótinum, L. = Lloyd'ia alpi'na, Salisb.

Anthoxanth'um, L. Grāmin'es, Grasses. Tri. Dig. od'ora'tum, L. 110. p. 5, 6. Sweet-scented Vernal-grass. Spring.g. Puélii, Lecoq and Lamotte. 9. a. 7.9. Annual Vernal-grass.

Anthrisc'us, Hoffm. Umbelli''ers, Umbellifers. Pent. Dig. Cérefol'ium, ${ }^{3}$ Hoffm. a. or b. 5-7. Garden Chervil.

sylvest'ris, "Hoffm. 102. b. 4-6. Wild or Cow C. Cow-parsley. vulga'ris, ${ }^{5}$ Pers. 76. b. 5, 6. Burr Chervil. Beaked Parsley. Com.C.

Anthyll'is, L. Legu'mo's $\mathbf{x}$, Leguminous Plants. Diad. Dec. Vulnera'ria, L. 105. p. 6-8. Kidney Vetch. Ladies'-finger. Woundwort. ${ }^{6}$

v. coccin'ea, Gray [A. Dille'nii, Sehult.]

Antirrhi'num, Tourn. Scro'pholarin'ese, Figworts. Did. Ang. Cymbala'ria, I. = Līnária Cymbalária, Mill.

Ělati'ne, L. = Līna'ria Ělati'na, Mill.

Linária, L. = Linna'ria vulgáris, Mill.

majjus, L. s. or p. 7-9. Common Snapdragon.

min'us, L. = Lina'ria visc'ida, Mnench.

Oront'ium, $\overrightarrow{\mathrm{L}}$. 47.a.7-9. Corn or Lesser Snapdragon. Calf's-snout. re'pens, L. = Lina'ria re'pens, Mill.

spur'ium, Sm. = Līna'ria spur'ia, Mill.

supínum, L. = Lina'ria supi'na. Desf.

Aparg'ia, Willd.

autumna'lis, Willd. = Leont'odōn autumna'lis, $\mathrm{L}$.

hirt'a, Hoffm. = Leont'odōn hirt'us, L.

hisp'ida, Willd. = Leont'odön hisp'idus, $\mathbf{L}$.

Tarax'aci, Willd. = Leont'odōn autumna'lis, v. pratens'is.

Ape'ra, P. de B. Grämin'es, Grasses. Tri. Dig.

interrupt'a, ${ }^{7}$ Beauv. 5. a. 6, 7. Dense-flowered Silky Bent-grass. ${ }^{8}$ Spi'ca-vent'i, ${ }^{9}$ Beauv. 18. a. 6, 7. Spreading Silky Bent-grass.

Ap'ium, L. Umbellif'ers, Umbellifers. Pent. Dig.

grav'eolens, L. 55. b. 6-8. Wild Celery. Smallage.

inunda'tum, ${ }^{10}$ Reichb.91. p. 6, 7. Least Water-Parsnip.Mudweed. ${ }^{11}$

v. Moor'ei, Syme.

no'diflo'rum, ${ }^{12}$ Reichb.82.p.7.8. Procumbent $W .-P$. Fool's W.-cress.

v. re'pens ${ }^{13}$ (Koch.) 1. Creeping Water-Parsnip.

v. ōchrea'tum, DC.

AP'ocřna'CE.E, Juss. Dogbanes.

Äquileg'ia, L. Raxcrc'ula'ces, Crowfoots. Polyan. Pent. vulga'ris, L. 57. p. 5-7. Columbine.

Ar'abis, L. Chucif'ers, Crucifers. Tetrad. Siliq. cil'ia'ta, R. Br. 1. b. 7, 8. Fringed Rock-cress.

v. hisp'ida, Syme.

hirsu'ta, R. Br. = A. sag'itta'ta, DC.

hisp'ida, L. $=\Lambda$. petræ'a, Lam.

per'folia'ta, ${ }^{14}$ Lam. 38. b. 5-7. Smooth Tower-mustard or Rockcress or Wall.cress.

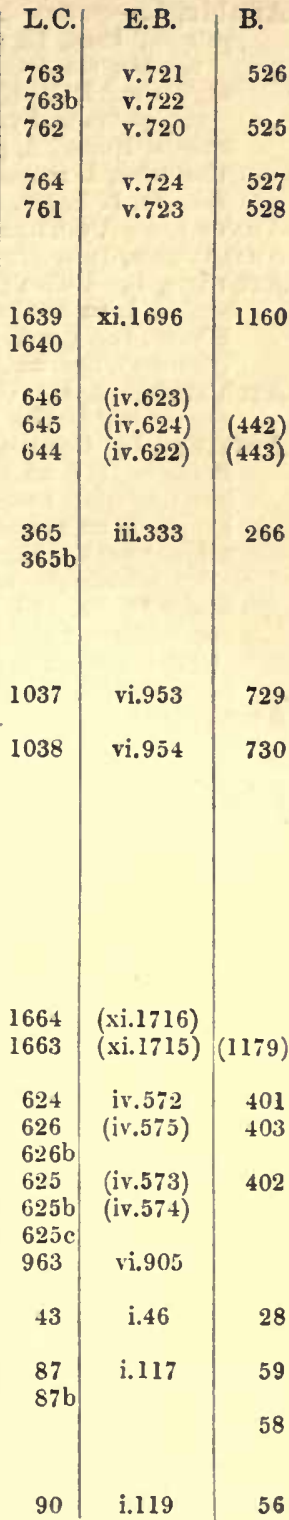

56

1 Authemis anglica.-A. maritima. 2 Maruta fotida. 3 Chærophyllum sativam.-Scandix Cerefolium. 4 Chærophyllum sylvestre. 5 C. Anthriscus, - Scandix Anthriscus. 6 Lamb's-toe. 7 Agrostis interrupta. 8 Wind-grass. 9 Agrostis Spica-renti.-Anemagrostis Spica-renti. 10 Helosciadium inundatum. - Sium inundatum. - Sison inundatum. "11 Water Marsh-wort. 12 Helosciadium nodiflorum,-Sium nodiflorum. $13 \mathrm{Helos-}$ ciadium repens. - H. nodiflorum, v, repens. - Sium repens. 14 Turritis glabra. 
Ar'abis, L.-continued. petræ'a, ${ }^{1}$ Lam. 15. p. 6-8. Alpine or Northern Rock or Wall-cress. v. hisp'ida, 1DC.

sag'itta'ta, ${ }^{2}$ DC. 91. b. 6-8. Hairy Rock or Wall-cress. v. glabra'ta, Syme.

strict'a, Huds. 2. p. 3-5. Bristol Rock-cress. Thal'ia'na, L. = Sisymb'rium Thal'ia'num, Hook.

Turri'ta, L. b. 5.7. Pendulous-podded W. Tower Turkey Pod. ${ }^{3}$ ArA'CEX, zee A'ROI'DEe.

ARAL'IA'CEE, Juss. Ivyworts.

Arb'utus, L. ER'îCA'CE正, Heathworts. Dec. Mon. alpi'na, $\mathrm{L}$. = Arct'ostaph'ylos alpi'na, Spr.

U'nedo, L. I. t. 9, 10. Strawberry Tree. Austere S. U'va-urs'i, Spr. = Aret'ostaph'ylos U'va-urs'i, Spr.

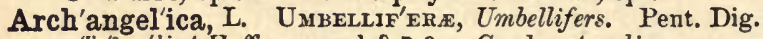
offic'ina'lis, ${ }^{4} \mathrm{Hoffm}$. p. or b ? 7-9. Garden Angelica.

Arct'ium, L. Compos'its, Composites. Syn. Aeq.

Barda'na, E.B. =A. min'us, schk.

intermed'ium, ${ }^{5}$ Lange. 25 ? b. 7, 8. Intermcdiate Burdock.

Lapp'a, L. = A. ma'jus, \& c. $^{\prime}$

ma'jus, ${ }^{6}$ Schk. 38. b. 8. Greater Burdock. Clot-bur.

v. sub'tōmento'sa, ${ }^{7}$ Lange. Downy-headed Burdock. min'us, ${ }^{8}$ Schk. 79. b. 8. Lesser Burdock.

nem'oro'sum, Lej. 18? b. 8. Narrow-leaved Burdock.

pu'bens, Bab. $=\mathbf{A}$. intermed'ium, Lange.

to'mento'sum, Bab.=A. ma'jus, v. sub'tōmento'sa.

Arct'ostaph'ylos, Adans. ER'1CA'CE alpi'na, ${ }^{\ominus}$ Spreng. 10. s. 5-7. Alpine Bearberry. Black B. $U^{\prime}$ va-urs'i ${ }^{10}$ Spreng. 31. s. 5, 6. Common Bearberry. Red B.

Ar'ena'ria, L. CAR'YopHYLL'Es, Cloveworts. Dec. Trig.

Cherle'ri, Benth. = A. sed'oï'des, Schultz.

cil'ia'ta, L. I. p. 6, 7. Fringed Sandwort.

fasc'icula'ta, Jacq.= Alsi'ne fasti'gia'ta, Bab. E.B.

fasti'gia'ta, Sm. = Alsi'ne fasti'gia'ta, Bab. E.B.

hirt'a, Wormsk. = A. sulca'ta, Schlecht.

leptoclad'os, Guss. =A. serpyll'ifol'ia, v. leptoclad'os.

mari'na, Sm. = Lěpigo'num margina'tum, Koch.

med'ia, L. = Lěpigo'num margina'tum, Koch.

norve'gica, Gunn. 1. p. 7, 8. Norwegian Sandwort.

peploïdes, ${ }^{11}$ L. 68. p. 5.8. Ovate-leaved Sea Purslane. ${ }^{12}$

rubell'a, Hook. = A. sulca'ta, Schl.

ru'bra, L. = Lěpigo'num ru'brnm, Fr.

sed'oi'des, ${ }^{18}$ Schultz. 8. p. 6-8. Mossy Cyphel.

serpyll'ifol'ia, L. 101. a. 6-8. Thyme-leaved Sandwort.

v. glu'tino'sa, Koch.

v. leptoclad'os ${ }^{14}$ (Guss.)

sulca'ta, ${ }^{15}$ Schlecht. 4. p. 7, 8. Alpine or Little Red Sandwort. ten'uifol'ia, ${ }^{16}$ L. 33, a. 6-8. Fine-leaved Sandwort.

v. lax'a (Jord.)

v. hyb'rida (Vill.)

trĭnerv'ia, ${ }^{17}$ L. 96. a. 5-7. Three-nerved or Plantain-leaved Sandwort.

üli'gino'sa, ${ }^{18}$ Schleich. 1. p. 5-7. Bog Sandwort. vern'a ${ }^{\prime}{ }^{9}$ L. 27. p. 5-7. Vernal Sandwort.

v. Gerard'i, Wahlb.

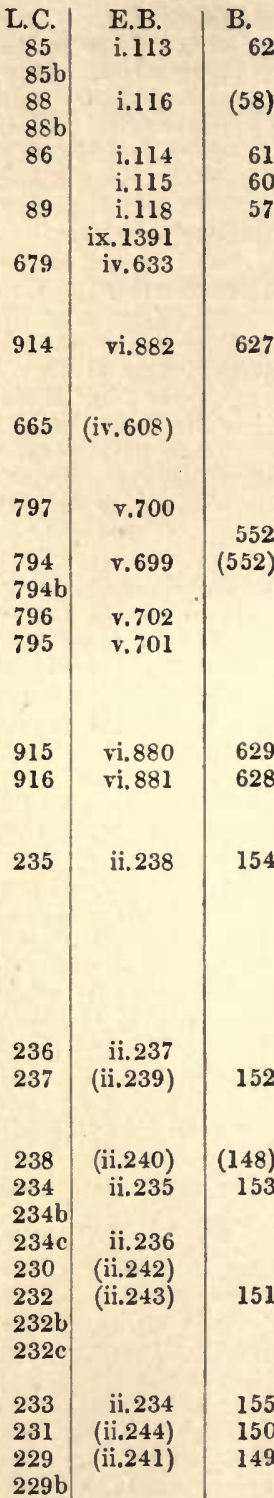

1 Arabis hispida,-Cardamine hastulata, 2 Arabis birsuta. - Turritis birsuta. 3 Touer Wall or Rockcress. 4 Angelica Archangelica. 5 Arctium pubens, 6 A. Lappa,-Lappa glabra. 7 Arctium tomentosum,Lappa tumentosa. 8 Arctinm Bardana. 9 Arbntus alpina. 10 A. Uva-ursi. 11 Honkenya peploides. 12 Sea Pimpernel, Chichweed or Sandwort. 13 Alsine Cherleria.-Arenaria Cherleri,-Cherleria sedoides. 14 Arenaria leptoclados. 15 A. rabella.-A. hirta.-Alsine rnbella, 16 Alsiae tenuifolia. 17 Mabriugia trinervis. 18 Alsine aliginoss.-A. stricta. 19 A. verna. 
Arist'oloch'ia, L. Arist'oloch'is'ce.e, Birthworts. Gyn. Hex. Cle'mati'tis, L. p. 6-8. Common Birthwort.

ARIST'OLOCH'IA'CEE, Juss, Birthworts.

Arme'ria, Willd. Plumbāaix'ex, Leadworts. Pent. Pentag. marit'ima, ${ }^{1}$ Willd. 108.p. 4-10. C. Thrift. Sea Pink or Gilliflower. r. pla'nifol'ia, ${ }^{2}$ Syme.

plantãgin'ea, ${ }^{3}$ Willd. C. p. 6-8. Plantain-leaved Thrift. rulga'ri-plantägin'ea. C. p.

vulga'ris, Benth. = A. marit'ima, Willd.

v. marit'ima $=$ A. marit'ims, Willd.

v. pla'nifol'ia $=$ A. marit'ima, v. pla'nifol'ia.

Armora'cia, Rupp.

amphib'ia, Koch = Nasturt'ium amphib'ium, R. Br.

rnstica'na, Rupp. = Cochlea'ria Armora'cia, $\mathrm{L}$.

Arnos'eris, Gærtn. Corpos'rтe, Composites. Syn. Eq. pusill'a, Gærtn. 23. a. 6, 7. Dwarf Nipple-wort. Swine's Succory. Lambs' Succory.

A'Roï'De.e, Juss. Arads.

Ar'rhenathe'rum, Beauv, Grāmn'es, Grasses. Tri. Dig. av'ena'ceum, ${ }^{5}$ Beauv. 110.p. 6, 7. False Oat-grass.

$r$. nodo'sum, ${ }^{6}$ Reichb.

bulbo'sum, Presl. =A. av'êna'ceum, v. nodo'sum.

èla'tius, M. and $K .=A$. av'ēna'ceum, Beauv.

Ar'temi'sia, L. Coxpos'its, Composites. Syn. Sup. Absinth'ium, L. 71. p. 8, 9. Com. Wormwood. Absinth. cæ'rulesc'ens, L, p. 8, 9. Bluish Mugwort. Lavender $M$. campest'ris, L. 4. p. 8, 9. Field Sonthernwood, or Wormwood. gall'ica, Willd. $=A$. marit'ima, v. gall'ica.

marit'ima, "L." 47. p. 8, 9. Drooping-flowered Sea Wormwood. ₹. gall'ica, ${ }^{7}$ (Willd.)

vulga'ris, L. 110. p. 7, 8. Mugwort.

$\checkmark$. coarcta'ta, Forcell.

Arthrolob'ium, Desv.

ebract'ea'tum, DC. = Orni'thopis ebract'ea'tus, Brot.

A'rum, L. A'roü'Dex, Arads. Monœe. Pol.

ital'icum, Mill. 6. p. 6. Italian Cuckoo-pint.

macula'tum, I. 80.p.4,5. Com. C. Wake-Robin. Arum. ${ }^{8}$

Arund'o, L.

ar'êna'ria, L. = Ammoph'ila arund'ina'cea, Host.

Cal'amagro'stis, L. = Cal'amagro'stis lanceola'ta, Roth.

Ep'igei'os, L. = Cal'amagro'stis Ep'igei'os, Roth.

Lappon'ica = Deyeux'ia neglect'a, v. Hook'eri.

Phragmi'tes, L. $=$ Phragmi'tes commu'nis, Trin.

strict'a, Schrad. = Deyeux'ia neglect'a, Kunth.

Asa'rum, L. Aris'tolochu'ces, Birthworts. Dod. Mon. europæe'um, L. 6. p. 5. Asa'rabac'ca.

Aspar'agus, L. Li'LIA'Cex, Lilyworts. Hex. Mon.

offic'ina'is, I. 5. p. 6-8. Asparagus.

v. hortens'is, Lond. Cat.

v. prostra'tus, Dum.

Asperu'go, 'Tourn. BorI gin'es, Borageworts. Pent. Mon. procumb'ens, L. a. 5-7. German Madwort. Madderwort.?

Asper'ula, L. RuB'in'CE.s, Stellates. Tetran. Mon. arvens'is, L. a. 6. Field Woodruff.

cy̆nanch'ica, L. 37. p. 6, 7. Small W. Squinancy-wort. od'ora'ta, L. 101. p. 5, 6. Sweet Woodruff.

tauri'na, L. p. 6. Four-leaved Woodmeff. Pink W.
L.C.

1238

1237

938

$938 \mathrm{~b}$

939

824

1462

1684

$1684 \mathrm{~b}$

77

773

774

$774 \mathrm{~b}$

772

$772 \mathrm{~b}$

E.B.

viii. 1250

riii. 1249

(vii.1152)

(vii. 1153 )

vii.1154

vii. 1155

vii.1152

vii. 1153

v.788

607

ix. 1391

(xi.1742)

1193

824

823

.

536

533

534

v. 735

v.732

535

1463

ix. 1393

1462

ix. 1392

941

1250

1237 viii. 1249

881

1390

ix. 1515

1030

$1390 \mathrm{a}$

$1390 \mathrm{~b}$

984

vii. 1120

707

708

707

705

706 iv. 662bis

iv. 661

iv. 660

iv. 662

I Armeria valgaris,-A. volgaris, v, maritima,-Statice armeris,-S, maritima. 2 Armeria valgaris, v, planifolia. 3 Statice plantaginea. I Hyoseris minims.-Lapsana pusilla. 5 Arena elstior.-Holeus arenaceus.-Arrhenathernm elatins. B A. bulbosum. 7 Artemisis gallica. 8 Lords and Ladies. 9 Trailing Catchweed. 
Aspid'ium, Sw.

acu'lea'tum, Sw. = Polystich'um loba'tum, Presl.

angula're, Willd. $=$ Polystich'um angula're, Presl.

crista'tum, Sw. = Lastræéa crista'ta, Presl.

di'lata'tum, Sw. = Lastræe'a di'lata'ta, Presl.

Fil'ix-fœ'mina, Sw. = Athyr'ium Fil'ix-fa'mina, Roth.

Fil'ix-mas, Sw. = Lastræ'a Fil'ix-mas, Presl.

loba'tum, Sw. = Polystich'um loba'tum, v. genui'num.

Lonchi'tis, Sw. = Polystich'um Lonchi'tis, Roth.

Ur'eopt'eris, Sw. = Lastræ'a Or'eopt'eris, Presl.

rig'idum, Sw. = Lastræ'a rig'ida, Presl.

spi'nulo'sum, Sw. = Lastræ'a spi'nulo'sa, Presl.

Thēlypt'eris, Sw. = Lastræáa Thēlypt'eris, Presl.

Asple'nium, L. Fin'ices, Ferns. Crypt. Fil.

acu'tum, Bory = A. Ad'ian'tum-ni'grum, v. acu'tum.

Ad'iant'um-ni'grum, L. 102. p. 6-9. Black-stalked Spleen-wort. ${ }^{1}$

v. obtu'sum, Kit. et Milde.

v. serpenti'ni, Koch.

v. acu'tum, ${ }^{2}$ Poll.

altern'ifol'ium, Wulf. $=\mathbf{A}$. germa'nicum, Weiss.

Cet'erach, L. = Cet'erach offic'îna'rum, Wilde.

Clermont'æ, Syme. p. 9. Lady Clermont's Spleenwort.

Fil'ix-fo'mina, Bernh. = Athyr'ium Fil'ix-f'e'mina, Roth.

fonta'num, ${ }^{3}$ Bernh. p. 6-9. Smooth Rock Spleenwort.

germa'nicum, ${ }^{+}$Weiss. 7. p. 6-9. Alternate-lcaved $S$.

lanceola'tum, Huds. 13. p. 6-9. Grcen lanceolate Spleenwort.

v. ob'ova'tum, Gren. \& Godr.

v. mi'crodōn, Moore.

mari'num, L. 50. p. 6-9. Sca Spleenwort.

v. acu'tum, Moure.

Ru'ta-mura'ria, L. 105. p. 6-10. Wall-rue Spleenwort.

v. ēla'tum, Moore.

v. pseud'o-germa'nicum, Milde.

septen'triona'le. Hull. 14. p. 6-10. Forked Spleenwort.

Trichom'anes, L. 108. p.5-10. Common Walt S. Maidenhair S.

v. an'ceps, Sol.

vir'ide, Huds. 41. p. 6-9. Green Maidenhair Spleenwort.

Ast'er, L. Compos'iтs, Composites. Syn. Sup.

Linos'yris, ${ }^{5}$ Bernh. 4. p. 8, 9. Flax-leaved Goldenlocks.

salig'nus, Willd. p. 9.

Tripol'ium, L. 65. p. 7-9. Michaelmas Daisy. Sca Stawwort. ${ }^{6}$

Astrag'alus, L. LeGU'mixo'se, Leguminous Plants. Diad. Dec.

alpi'nus, L. 3. p. 7. Alpine Milk-Vetch.

campest'ris, DC. $=$ Oxyt'ropis campest'ris, DC.

glycyphyll'os, L. 63. p. 6-9. Sweet Milk-Vetch. Liquorice Vetch. Hy̆poglo'ttis, L. 39. p. 6, 7. Purple Mountain Milk-Vetch̆.?

1770
$1770 \mathrm{~b}$
$1770 \mathrm{c}$

xii.1874

L.C. E.B.

B.

1279

1283

1282

1278

1281

1285

1284

1280

$1770 \mathrm{~d}$ xii. 1875

1292

xii. 1879

1768

1776

xii. 1872

1286

1287

1769

xii. 1881

1294

$1769 \mathrm{~b}$

$1769 \mathrm{c}$

1771

$177 \mathrm{lb}$

1775

$1775 \mathrm{~b}$

$1775 \mathrm{c}$

1777

xii. 1873

1288

xii. 1876

1289

xii. 1880

1293

xii. 1882

1295

1773

xii. 1878

1290

1772

xii. 1877

1291

731

v.777

496

729

730

v. 776

495

371

iii. 375

268

373

iii. 377

269

u'rālens'is, L. = Oxyt'ropis u'rālens'is, DC.

Astrant'ia, L. UMBeLLIF'ERA, Umbellifers. Pent. Dig. ma'jor, L. p. 6, 7. Greater Astrantia.

Ath'amant'a, $\mathbf{L}$.

Lib'ano'tis, Sm. = Ses'eli Lib'ano'tis, Koch.

Athyr'ium, Roth. Fin'ices, Ferns. Crypt. Fil.

alpest're, ${ }^{8}$ Milde. 10. p. 7, 8. Alpine Lady-fern or Polypody. r. obtusa'tum, Syme.

Fil'ix-fœ'mina, ${ }^{9}$ Roth. 110. p. 6,7. Lady-fern. L. Spleenwort. ${ }^{10}$

v. erect'um, Syme.

v. Watso'ni, Syme.

1 Black Maidenhair Spleenwort. 2 Asplenium acntum. SAthyrinm fontanum. 4 Asplenium alternifolium. 5 Chrysocoma Linosyris.-Linosyris vulgaris. 6 Sea-side Aster. 7 Tongue-under-tongue. 8 Poly. podium alpestre.-Pseudothyrinm alpestre. 9 Aspidium Filix-fœemina.-Asplenium Filix-fœmina. io Female Shield Fern. 
Athyr'ium, Roth.-continued.

flex'ile, ${ }^{2}$ Syme. p. 6-9. Flexile Lady-fern.

fonta'num, Presl. = Asple'nium fonta'num, Bernh.

Atrip'lex, T. CuE'NOPOD'IA'CEs, Chenopods. Polyg. Monoc. angust'ifol'ia, Sm. $=$ A. pat'ula, v. angust'ifol'ia.

ar'ëna'ria, Woods = A. lac'inia'ta, $\mathrm{L}$.

Babingto'nii, Woods. 67. a. 7-9. Babington's Orache. ${ }^{2}$ v. viresc'ens, Lange.

deltoïdea, Bab. a. 7-10. Triangular-leaved Orache.

v. prostra'ta, ${ }^{3}$ Bab.

erect'a, Huds. $=$ A. pat'ula, v. erect'a.

far'ino'sa, Dum. = A. lac'inia'ta, L.

hasta'ta, L. a. 6-10. Halberd-leaved Orache.

hortens'is, L. a. 7, 8. Garden Orache. Mountain Spinach. lac'inia'ta, L. 39. a. 7-10. Frosted Sea Orache.

littora'lis, L. 37. a. 7-9. Grass-leaved Sea Orache.

v. $\operatorname{mari}^{\prime} a^{3}(\mathrm{~L}) 14$.

mari'na, $\mathrm{L} .=\mathrm{A}$. littora' lis, v. mari'na.

nit'ens, Reb. a. 8, 9. Shining-leaved Orache.

pat'ula, L. a. 6-10. Narrow-leaved Orache.

₹. erect'a, ${ }^{6}$ Huds.

v. angust'ifol'ia, ${ }^{7} \mathrm{Sm}$.

pedunc'ula'ta, ${ }^{8}$ L. 7. a. 8-10. Stalked-fruited Sea Orache.

\begin{tabular}{|l|l|}
\hline $\begin{array}{l}\text { L.C. } \\
1780\end{array}$ & E.B. \\
& xii. 1871 \\
& \\
1196 & viii.1207 \\
$1196 \mathrm{~b}$ & viii.1206 \\
1195 & viii.1204 \\
$1195 \mathrm{~b}$ & \\
& \\
1194 & viii.1204, \\
1197 & (viii. 1207$)$ \\
1192 & viii.1200 \\
$1192 \mathrm{~b}$ & viii. 1201 \\
& \\
1193 & viii. 1202,3 \\
$1193 \mathrm{~b}$ & viii.1203 \\
$1193 \mathrm{c}$ & viii. 1202 \\
1199 & viii.1209 \\
\hline 1198 & vili.1208
\end{tabular}

B.

port'ula'coï'des, ${ }^{9}$ L. 35. s. 8-10. Sea Purslane. Shrubby Orache.

ros'ea, L. = A. lac'inia'ta, L.

Smith'ii, Syme. a. 8, 9. Smith's Orache.

triang'ula'ris, Willd. $=\mathbf{A}$. deltoï'dea, v. prostra'ta.

At'ropa, L. So'Lĩxa'ce E, Nightshades. Pent. Mon.

Belladonn'a, L. 33. p. 6.8. Deadly Nightshade. Dwale.

Ave'na, L. Grínun'ex, Grasses. Tri. Dig.

alpi'na, Sm. = A. pratens'is, v. alpi'na.

ëla'tior, L. = Ar'rhenathe'rum av'êna'ceum, Beauv.

fat'ua, L. 76. a. 6-8. Wild Oat. Haver.

v. pil'osiss'ima, Gray.

r. intermed'ia (Lindgr.)

flaresc'ens, L. = Trise'tum fiaresc'ens, Beauv.

pla'niculm'is, Schrad. p. 7. Flat-stemmed Oat-grass.

pratens'is, L. 73. p. 6, 7. Glabrous 0 . Narrow-leaved $0 .{ }^{10}$

v. alpi'na ${ }^{11}(\mathrm{Sm}$.)

v. longifol'ia (Parn.)

pubesc'ens, ${ }^{12}$ Huds. 85. p. 6,7. Downy Oat-grass.

strigo'sa, Schreb. a. 6, 7. Black Oat. Bristle-pointed Oat.

Azal'ea, L.

procumb'ens, L. = Loiseleu'ria procumb'ens, Desr.

Ballo'ta, L. LAB' IA're, Labiates. Did. Gym.

foe'tida, Lam. = B. ni'gra, v. alb'a.

ni'gra, L. 76. p. 7, 8. Black Horehound.

v. fo'tida $=$ v. alb'a.

v. $\operatorname{alb}^{\prime} a^{13}$ (L.)

v. ru'dera'lis, ${ }^{14}$ Koch.

ru'dera'lis, Sw. = B. ni'gra, v. ru'dera'lis.

Barbare'a, R. Br. Crdcrf ere, Crucifers. Tetrad. Siliq. arcua'ta, Reichb. b. 6. Reichenbach's Yellow-Rocket. intermed'ia, Boreau. 10. b. Intermediate Yellow-Rocket. pree cox, ${ }^{15}$ R. Br. b. 4-10. American Cress. Early Winter C. strict'a, Andrz. 10. b? 5-8. Small-flowered Yellow Rocket.

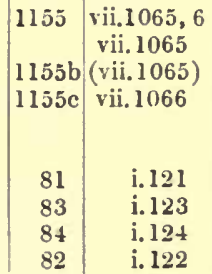

1 Polypodium flexile.-Psendothyrium flexile. 2 Spreading-fruited Orache. S Atriplex triangularis. 4 Atriplex arenaria.-A. farinosa.-A. rosea. ${ }^{3}$ A. marins. 6 A. erecta. 7 A. angustifolis. 8 Obione pedunculata. 90 . portulacoides. 10 Perennial Oat. 11 Avena alpina. 12 Trisetnm pubesceos. 13 Ballota fiptids.-B. pigra, v. foetida. 14 B. ruderalis. 15 Erysimum proecox. 
Barbare'a, R. Br.-continued. vulga'ris, ${ }^{1}$ R. Br. 95. b. or p. 4-10. Common Yellow Rocket. ${ }^{2}$

Barts'ia, L. Scrópuularin'es, Figworts. Did. Ang.

alpi'na, L. 8. p. 6-8. Alpine Bartsia. Alpine Painted-cup.

Od'onti'tes, ${ }^{3}$ Huds. 111. a. 6-8. Red Bartsia. Red Eye-bright.

v. vern'a, Reichb. 3.

v. sero'tina, Reichb.

v. diverg'ens, Balb.

visco'sa, ${ }^{4}$ L. 19. a. 6-10. Yellow viscid B. Marsh Painted Cup.

Bell'is, L. Compos'it x Composites. Syn. Sup. perenn'is, L. 112. p. 1-12. Common Daisy.

Berberiv'Ee, Vent. Berberids.

Ber'beris, L. Berberid'es, Berberids. Hex. Mon. vulga'ris, L. 77. s. 5, 6. Barberry.

Be'ta, Tourn. Che'nopod'IA'Ces, Chenopods. Pent. Dig. marit'ima, ${ }^{5}$ L. 35. a. 6-10. Sea Beet. Common Beet. vulga'ris, $\mathrm{L}_{*}=\mathrm{B}$. marit'ima, $\mathrm{L}$.

Beto'nica, $\mathbf{L}$. offic'îna'lis, L. = Stach'y̆s Beto'nica, Benth.

Be'tula, Tourn. Co'pulfíter.e. Monce. Pol. alb'a, L. 107. t. 4, 5. Birch.

al'nus, L. = Aln'ıs glu'tino'sa, Gærtn.

glu'tino'sa, Fr. 53. t. 4, 5. Common Birch.

v. de'nuda'ta, Hook.

v. pubesc'ens (Wallr.)

na'na, L. 15. s. 5. Dwarf Birch. pend'ula, Roth $=$ B. verruco'sa, Ehrh. verruco'sa, ${ }^{6}$ Ehr. t. 4, 5. White Birch.

Bidens', L. Compos'ıx s, Composites. Syn. Aq. cern'ua, L. 81. a. 7-10. Nodding Bur-Marygold. v. radia'ta, Sond.

triparti'ta, L. 80. a. i-9. Tripartite Bur-Marygold.

Blacksto'nia, Huds. Gentia' xe. Gentian-worts. Oct. Mon. perfolia'ta, ${ }^{7}$ Huds. 60. a. 7-9. Yellow Centaury. ${ }^{8}$

Ble'chnum, L.

borea'le, Swartz = Loma'ria spi'cant, Desv. spi'cant, Roth = Loma'ria spi'cant, Desv.

Blys mus, Panz.

compress'us, Panz. = Scirp'us Ca'ricis, Retz.

ru'fus, Link = Scirp'us ru'fus, Schrad.

Boñ̄an'Ex, DC. Borageworts.

Bora'go, Tourn. BorĀgrixe, Boragcworts. Pent. Mon. offic' ina'lis, L. b. 6, 7. Common Borage.

Borkhaus'ia, L.

fo'tida, DC. = Cre'pis fo'tida, $\mathrm{L}$.

tarax'acifol'ia, DC. = Cre'pis tarax'acifol'ia, 'Thnill.

Botrych'ium, Swartz. Fil'ices, Ferns. Crypt. Fil.

(lanceola'tum, Angström)?

Luna'ria, ${ }^{8}$ Swartz. 96. p. 6-8. Moonwort.

v. inci'snm, Milde.

ruta'ceum, Swartz. p. 8.

Brach'ypod'ium, Bealv. GRĀurs'es, Grasses. Tri. Dig. pinna'tum, ${ }^{10}$ Beauv. 35 . p. 7. Barren False-Brome-grass. ${ }^{11}$ v. pubesc'ens, Syme. sylva'ticum, ${ }^{12}$ R. \& S. 105. p. 6, 7. Slender or Wood FalseBrome-grass.

v. glabresc'ens, Syme.

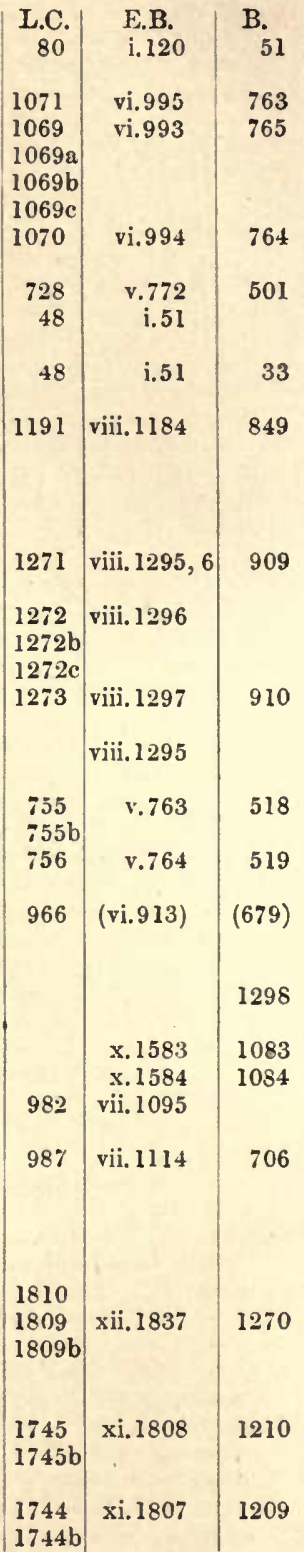

B.

763

65

764

501

33

49

910

518

519

298

1083

$\mathrm{x} .1584 \quad 1084$

706

1270

1210

1209

1 Erysimum Barbarea, 2 Belleisle Cress._Bitter Winter Cress._Herb St. Barbara. 3 Euphrasin Odontites,-Odontites rubra. 4 Eufragia viscosa. 5 Beta vulgaris. 6 Betula pendula, 7 Chlora perfoliata. 8 Perfoliate Yellow-uort. 9 Osmnnda Lunaria. 10 Bromus pinastus.-Festucs pinnata. 11 Heath Brome-grass, 12 Bromus sylvaticus. 
Brass'ica, L. Crucir'ere, Crucifers. Tetrad. Siliq. adpress'a, ${ }^{1}$ Boiss. C. b. 7, 8. Hoary Mustard. alb'a, ${ }^{2}$ Boiss. 80, a. 6, 7. White Mustard. brer'ipes, Syme.

campest'ris, L. $=$ B. ru'taba'ga, DC.

Cheiranth'ns, ${ }^{3}$ Vill. C. p. $i, 8$. Tall Wall-flower Cabbage. E'rucast'rum, Vill.

L.C.

1.30

132

monens'is, ${ }^{4}$ Huds. 16. p. 5, 6. Duarf Wall-Alower Cabbage. ${ }^{3}$ mura'lis, Boiss. = Dip'lotax'is mura'lis, DC.

Na'pus, L.? a. or b. 5, 6. Wild Rape. Cole-seed.

ni'gra, ${ }^{6}$ Koch. 62, a. 6-9. Black, or Brown Mustard.

ol'era'cea, L. 11. b. or p. 5-8. Sea Cabbage, Kale, or Colewort. ${ }^{7}$ or'ienta'lis, L. = Erys'imum or'ienta'le, R. Br.

polymorph'a, Syme.

Ra'pa, L. a. or b. 4-7. Wild Turnip.

v. sati'va, H. C. Wats.

v. sylrest'ris, H. C. Wats.

r. Briggs'ii, H. C. Wats.

Rutaba'ga, DC.? a. 6-9. Wild Navew. Swedish Turnip. ${ }^{9}$

Sina'pis, ${ }^{10}$ Visiani. 111, a. 5, 6. Charlock, Wild, or Corn Must. ${ }^{11}$

Sinapist'rum, Boiss. = B. Sina'pis, Visiani.

ten'uifol'ia, Boiss. = Dip'lotax'is ten'uifol'ia, DC.

vīmin'ea, Boiss. a. Small Sand Rocket.

Bri'za, L. Grämu'Ex, Grasses. Tri. Dig.

max'ima, L.

med'ia, L. 105. p. 6. Quaking-grass. Maiden-hair.

min'or. L. 7. a. 7. Small Quaking-grass.

Brom'us, L. Gramines, Grasses. 'Iri. Dig.

arvens' is, ${ }^{12}$ L. a. or b. 7,8 . Taper Field Brome-grass.

asp'er, Murr. [ramo'sus, Huds.] 93.p. 6,7. Hairy Wood or RoughB.

v. sero'tinus (Benek.)

v. Beneken'ii (Syme)

commuta'tus, ${ }^{18}$ Schrad. 85.b. 6, 7. Confused Brome-grass. ${ }^{14}$

v. pubesc'ens, Lond. Cat.

v. multiflo'rus (Parn.)

dĭand'rus, Curt. $=$ B. ma'dritens'is, $\mathrm{L}$.

erect'us, Huds. 44. p. 6, 7. Upright Perennial Brome-grass.

v. villo'sus, Bab.

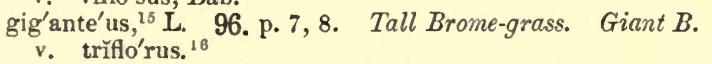

ma'dritens'is, ${ }^{17}$ L. 11. a. or b. 6, 7. Upright Annual B. Compact $B$.

v. Curti'sii, Bab.

v. rig'idus (Roth)

max'imus, Desf. C. b. or a. 6, 7. Great Brome-grass.

moll'is, ${ }^{18}$ L. 111. p. 5-7. Soft Brome-grass.

v. glabresc'ens, Coss.

v. Lloydi'anus, Syme.

v. hordea'ceus, Fr.

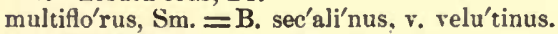

pat'ulus, ${ }^{19}$ Kocb. a. 6. Spreading Brome-grass.

pinna'tus, $\mathrm{L}$. = Brach'y pod'ium pinna'tum, Beauv.

pratens'is, Ehrh. = B. Commuta'tus, Schrad.

rac'emo'sus, ${ }^{20}$ "L." b. 6, 7. Smooth Ficld B. Racemose $B$.

sec'ali'nus, ${ }^{21}$ L. 80 . b. or a. 6, 7. Smooth Rye Brome-grass.

v. velu'tinus ${ }^{22}$ (Schrad.) Many-flowered Field B. Downy Rye B. $|1739 \mathrm{~b}|$

E.B.

i. 86

B.

83

80

i. 84

i. 94.5

i. 89

128

i. 92

79

133

127

124

129

123

126

$126 \mathrm{a}$

$126 \mathrm{~b}$

$126 \mathrm{c}$

125

131

(i.89)

(i.83)

i.91

77

i. 94

i. 88

i. 85

i. 87

82

78

i. 88.90

i. 90

i. 83

(79)

(81)

i. 93

81

i. 95

1697

1698

xi. 1774

1226

xi.1775

1227

1743

xi. 1806

1216

xi. 1795

1212

$1733 a$

$1733 \mathrm{~b}$

$17+1$

$17+1 b$

$17+1 \mathrm{c}$

1734

$1734 \mathrm{~b}$

1732

xi. 1796

1211

xi. 1793

1217

xi.1794

1735

xi. 1797

1215

$1735 \mathrm{a}$

$1735 \mathrm{~b}$

1737

xi. 1798

1742 xi. 1804

1214

$17+2 \mathrm{~b}$

$1742 \mathrm{c}$

xi. 1805

$1742 \mathrm{~d}$

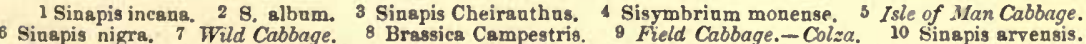
- Brassica Sinapistrum. 11 Kedlock.-Runch. 12 Serrafalcus arvensis. 13 Bromus pratensis,-Serrafalens commutatus. 14 Tumid Field Brome-grass. 15 Festues gigantea. is F. triflora, 17 Bromus diandrus. 16 Serrafalcus mollis. $19 \mathrm{8}$. patulus, $20 \mathrm{8}$. racemosus. $21 \mathrm{8}$. secalinus. 22 Bromus multiforus.-B. velutinus. 
Brom'us, L.-continued.

squarro'sus, ${ }^{1}$ L. a. 6, 7. Corn Brome-grass.

ster'ilis, L. 102. b. or a. 6, 7. Barren Brome-grass.

sylva'ticus, Sm. = Brach'ypod'ium sylva'ticum, R. \& S.

tecto'rum, L.

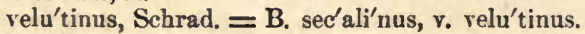

Brunell'a, L.

vulga'ris, $\mathrm{L} .=$ Prunell'a vulga'ris, $\mathbf{L}$.

Bryo'nia, L. Cucurb'rta'ces, Cucurbits. Monœc. Pent. dioic'a, Jacq. 59. p. 5-9. Red-berried Bryony.

Buffo'nia, L. CAR'YoPHYL'Ex, Cloveworts. Tetran. Dig. ann'ua, DC. = B. ten'uifol'ia, Sm.

Bu'nias. ten'uifol'ia, ${ }^{2} \mathrm{Sm}$. a. 6. Anmual Buffonia.

Caki'le, L. = Caki'le marit'ima, Scop.

Bu'nium, DC.

Bulb'ocast'anum, L. = Car'um Bulb'ocast'anum, Koch. flexuo'sum, With. = Co'nopod'ium de'nuda'tum, Koch.

Bupleu'rum, L. Umbellif'zrs. Umbellifers. Pent. Dig. ar' ista'tum, ${ }^{3}$ Bartl. 2. a. 7. Narrow-leaved Hare's-ear. falca'tum, L. 2. p. 8. Falcate-leaved $H$. Long-leaved $H .{ }^{4}$ od'onti'tes, Sm. = B. ar'ista'tum, Bartl. rotund'ifol'ium, L. 39. a. 6,7. Common Hare's-ear. Perfoliate H. ${ }^{5}$ ten'uiss'imum, L. 23. a. 8, 9. Slender Hare's-ear.

Bu'tomus, Tourn. Ax'rsma'CE, Alismads. Enn. Hex. umbella'tus, L. 59. p. 6, 7 Flowering Rush.

Bux'us, Tourn. EcPhori'ra'CEx, Spurgeworts. Monœc. Tet. semp'ervir'ens, L. s. 4, 5. Box Tree.

Cakile, Tourn. Crucif'ers, Crucifers. Tetrad. Silic. marit'ima, ${ }^{6}$ Scop. 59. a. 6, 7. Purple Sea Rocket. v. in'tegrifol'ia, Koch.

Cal'amagro'stis, Adans. Grämrn'Ex, Grasses. Tri. Dig. ep'igei'os, ${ }^{7}$ Roth. 57. p. 7, 8. Wood Small-reed.

lanceola'ta, ${ }^{8}$ Roth. 38. p. 7,8. Purple flowered Small-reed. lappon'ica, Hook. = Deyeux'ia neglect'a, v. Hook'eri. strict'a, Nutt. = Deyeur'ia neglect'a, Kunth.

v. Hook'eri = Deyeux'ia neglect'a, v. Hook'eri.

Cal'aminth'a, Moench. LAв'rA'т., Labiates. Did. Gym. Ac'inos, Clairr. = C. arvens'is, Lam. arvens' is, ${ }^{9}$ Lam. 74. a. or b. 7, 8. Basil-Thyme. Field Calamint. Cli'nopod'ium, ${ }^{10}$ Benth. 85. p. 7-9. Wild Basil. Hedge C. menthiful'ia, Host. = C. offic'ina'lis, Moench.

v. Briggs'ii $=$ C. offic'īna'lis, v. Briggs'ii.

Nep'eta, ${ }^{11}$ Clairv. 8. p. 7, 8. Lesser or Small-flowered Calamint. offic'ina'lis, ${ }^{12}$ Moench. 61. p. 7-9. Common Calamint. v. Briggs'ii, ${ }^{13}$ Syme.

sylva'tica, Bromf. 3. p. 8-10. Wood Calamint.

Callit'richē, L. Halora'Ge e, Hippurids. Monce. Mon. aqua'tica, $\mathrm{Sm} .=\mathrm{C}$. verna'lis, Koch.

autumna'lis, L. 27. p. 6-10. Autumnal Water Star-wort.

ha'mula'ta, Kuetz. p. 6-10. Hooked Water Star-wort.

v. pedunc'ula'ta, ${ }^{1}$ (DC.) Stalked $W . S$.

obtusang'ula, Le Gall. 27. Obtuse-fruited W. S.

v. Lach'ii (Warren)

pedunc'ula'ta, DC. = C. ha'mula'ta v. pedunc'ula'ta.

1 Serrafalcus squarrosus. 2 Bnffonia annua. 3 Bupleurum odontites. 4 Sickle-leaved Hare'sear. 6 Thorough-wax. 6 Bunias Cakile. 7 Arundo Epigeios. 8 A. Calamagrostis. 9 Acinos rulgaris.-Calamintha Acinos.-Melissa Acinos.--Thymus Acinos. 10 Clinopodium vulgare.-Melissa Clinopodium. 11 M. Nepeta. -Thymus Nepeta. ${ }^{2}$ Calamintha menthifolia,-Thymus Calamintha.-Melissa Calamintha. 13 Calamintha monthifulia, v. Briggsii. 14 Callitriche pedunculata. 
Callit'richē, L.continued.

plătycarp'a, Kuetz. $=$ C. stagna'lis, Scop.

Stagnn'lis, ${ }^{1}$ Scop. 93. p. 5-12. Large-fruited Water Star-wort. trunca'ta, Guss. 1. 5-10.

vern'a, $L$.

verna'lis, ${ }^{2}$ Koch. p. 6-10. Vernal Water Star-wort.

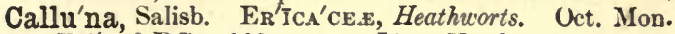

Eri'ca, ${ }^{3}$ DC. 110. s. 7-9. Ling, Heather.

v. glabra'ta, Seem.

v. inca'rua, Auct.

vulga'ris, Salisb. = C. Eri'ca, DC.

Calth'a, L. Raxunc'uld'ces, Crowfoots. Polyan. Pol.

palus'tris, L. 111. p. 3-5. Marsh Marigold. Water Caltrops. ${ }^{4}$

v. vulga'ris (Schott)

v. Guerange'rii (Boreau)

v. min'or, Syme.

radi'cans, Forster. p. 5, 6. Creeping Marsh Marigold.

Calysteg'ia, R. Br. Coxvolv' duA'CE.E, Bindweeds. Pent. Mon. se'pium, ${ }^{3}$ R. Br. 93. p. 6-8. Large or Hooded Bindweed.

Sol'danell'a, ${ }^{6}$ R. Br. 44. p. 6-8. Sea Bindweed.

Came'lina, Crantz. Crucir'zre, Cmucifers. Tet. Silic. fo'tida, Fr. = C. sati'va v. fo'tida.

sati'va, ${ }^{\top}$ Crantz, a. 6, 7. Cultivated Gold-of-Pleasure.

v. fo'tida, ${ }^{8}$ Fr. Fetid Gold-of-Pleasure.

Campa'nula, L. Campánolaces. Bellworts. Pent. Mon. glom'era'ta, L. 49. p. 9, 10. Clustered Bell-flower. v. nána, C. Bailey.

hèdera'cea, L. $=$ Wahlenberg'ia bědera'cea, Reichb.

hyb'rida, L. = Spěcula'ria hyb'rida, DC.

la'tifol'ia, L. 59. p. 7,8. Giant Bell-flower.

pat'ula, L. 28. p. or a. 7-9. Spreading Bell-flower.

pers'icifol'ia, L. p. 7, 8. Peach-leaved Bell-flower.

rapunc'uloï'des, L. 24. p. 7, 8. Creeping Bell-flower.

Rapunc'ulus, L. 31. b. 7, 8. Rampion Bell-flower.

rotund'ifol'ia, L. 110. p. 7-9. Harebell. Round-leaved Bellflover. Bluebell.

v. lancifol'ia, Koch.

v. spec'io'sa, More.

Trache'lium, L. 58. p. 9, 10. Nettle-leaved Bell-flower.

Campa'xula'Ce. Juss. Bellworts.

L. C.

582

$5 \leqslant 6$

581

918

$918 \mathrm{a}$

$918 b$

37

$37 \mathrm{a}$

$37 \mathrm{~b}$

$37 \mathrm{c}$

38

1008

1009

(vi.924)

(vi.925)

$(684)$

$(685)$

i. 142

121

$121 \mathrm{~b}$

i. $1+1$

(i. 142)

901

$901 \mathrm{~b}$

vi. 866

vi. 875

vi. 874

903

908

906

904

907

905

$905 \mathrm{~b}$

$905 \mathrm{c}$

902

895

Can'nabis, Tourn. Urtīca'ces, Nettleworts. Diœc. Pent. sati'va, L. a. 7-9. Hemp.

CAP'RIFOL'ia'Ce.e, Kunth. Caprifoils.

Cap'rifol'ium, Tourn.

per'folia'tum, Roehl. = Lonice'ra Cap'rifol'ium, L.

Periclym'enum, Roem. \& Sch. = Lonice'ra Periclym'enum, L.

Capsell'a, Moench. Crucif'er., Crucifers. Tetrad. Siliq.

Burs'a-pasto'ris, ${ }^{9}$ Mœnch. 112. a. 4-11. Shepherd's Purse. Cass-

Cardami'ne, L. Crucir'er.e, Crucifers. Tetrad. Siliq. [weed. ${ }^{10}$ ama'ra, L. 71. p. 4, 6. Large-flowered Bitter-Cress. ${ }^{11}$

bell'idifol'ia, L. p. 7-10. Daisy-leaved Lady's Smock. [root. ${ }^{13}$ bulbif'era, ${ }^{12}$ R. Br. 10. p. 4-6. Bulbiferons Coralwort, or Coralflexuo'sa, ${ }^{14}$ With. 88. a. or b. 4-9. Wood Hairy Lady's Smock.

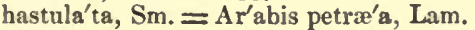

hirsu'ta, L. 106. a. 4-9. Lady's Smock. Hairy Bitter Cress. impat'iens, I. 26. b. or 4. 5-8. Impatient-podded L. $S_{.}{ }^{15}$ pratens'is, L. 111. p-4.6. Meadow Lady's Smock. Cuckoo Flower. ${ }^{16}$

C. platycarpa. 2 C. aquatica. 3 Calluna valgaris.-Erica valgaris. 4 Mendow-Rout. ${ }^{3}$ Couvolvalus sepium. 6 C. Soldanella, 7 Alyssum satirs. 8 Camelina fotida. 9 Thlaspi Bursa-pastoris, 10 Poor Man's Parmacetic.-St. James's Weed. ${ }_{11}$ Bitter Lady's Smock. 12 Dentaria bnlbifera, ${ }_{13}$ Tooth-cress.-Toothwort. 14 Cardamine sylvatica. 15 Narrow-leaved Bitter-cress. 16 May-flower. 
Cardami'ne, L. continued.

v. denta'ta, Hayne \& Welwitsch.

v. Haynea'na, Welwitsch.

sylva'tica, Link = C. flexuo'sa, With.

Card'uus, L. Compos'rte, Composites. Syn. Eq. acanth'oídes, $\mathrm{L} .=\mathrm{C}$. crisp'us, L.

acaul'i-pratens' is = Cni'cus Woodward'ii (hybrid).

acaul'is, L. $=$ Cni'cus acaul' is, Willd.

arvens' $\mathrm{i}$-acaul'is $=$ Cni'cus Clark'ei (hybrid).

arvens' is, Curt. $=$ Cni'cus arrens'is, Willd.

v. seto'sus $=$ Cni'cus arvens'is, v. seto'sus.

crisp'us, ${ }^{1}$ L. 87. b. 6-8. Welted Thistle.

v. polyanth'emos, Gren.

v. acanth'oï'des, L.

ĕrioph'orus, L $=$ Cni'cus ěrioph'orus, Roth.

het'erophyll'us, L. = Cni'cus het'erophyll'us, Willd.

lanceola'tus, L. $=$ Cni'cus lanceola'tus, Willd.

Maria'nus, L. = Sil'ybum Maria'num, Gærtn.

nu'tans, L. 73, a. 7-9. Musk Thistle.

nu'tans $\times$ crisp'us. Hybrid betw. Musk and Welted Thistles.

palust'ris, L. $=$ Cni'cus palust'ris, Willd.

pratens'i-palust'ris, Syme = Cni'cus For'steri (hybrid).

pratens'is, Huds. = Cni'cus pratens'is, Willd.

pyc'noceph'alus, ${ }^{2}$ Jacq. 70. a. or b. 6-8. Slender-flowered $T$.

ten'uiflo'rus, Curt. = C. pyc'noceph'alus, Jacq.

tu'bero'sus, L. = Cni'cus tu'bero'sus, Roth.

Ca'rex, L. Crp'ERs'CEx, Sedges. Monoe. Tri.

acu'ta, L. 64. p. 5-6. Slender-spiked or Acute Sedge.

v. prolix'a (Fr.)

v. trǐcosta'ta (Fr.)

v. gracilesc'ens, Almq.

alpi'na, ${ }^{3}$ Sw. 2. p. 8. Close-headed Alpine Sedge.

ampulla'cea, Good. $=$ C. rostra'ta, Stokes.

aqua'tilis, Wahl. 15. p. 6.8. Straight-leaved Water Sedge."

v. Watso'ni, Syme.

v. cuspida'ta, Læstad.

v. ep'igei'os, Lestad.

ar'ēna'ria, L. 67. p. 6. Sea-side Sedge. Sand Carex.

v. liger'ica (J. Gry). 2.

atra'ta, L. 8. p. 7. 8. Black Sedge.

axilla'ris, Good. 37. p. 6. Axillary-clustered Sedge.

bĭnerr'is, Sm. 93. p. 6, 7. Green-ribbed Sedge.

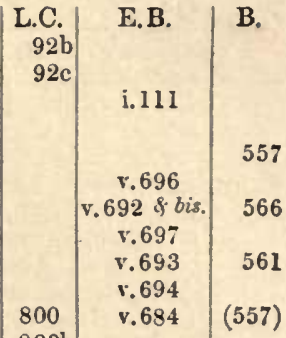

$800 \mathrm{~b}$

$800 \mathrm{c}$

v. 687

v.691

562

v. 686

563

559

799

ง. 683

555

799

v. 685

r. 688

v. 695

v. 690

798

(v.682)

v. 682

v. 689

556

560

565

558

564

1582

x. 1639

1123

$1582 \mathrm{~b}$

$1582 \mathrm{c}$

$1582 \mathrm{~d}$

1578

$\mathrm{x} .1636$

1124

x.1680 1148

1585

x.1641

$1585 \mathrm{~b}$

$\mathrm{x} .1642$

Boenninghausia'na, Weihe. 15. p. 6. Bönninghausian's $S$.

$1585 \mathrm{c}$

1561

$\mathrm{x} .1618$

1118

$1561 \mathrm{~b}$

1579

1571

x. 1635

1126

x.1628 1114

bri'zoi'des, L. p. 7.

Buxbaum'ii, Wahl. I. p. 7. Hoary Sedge. Buxbaum's Carex.

$\mathbf{x} .1667$

x.1629

crspito'sa, Gay $=$ C. strict' $a$, Good.

crespito'sa, Good. = C. Goodenow'ii, J. Gay.

canesc'ens, L. $=$ C. curt'a, Good.

capilla'ris, L. 15. p. 6, 7. Dwarf Hair-like Sedge. Capillary S. clandesti'na, Good. $=$ C. hum'ilis, Leysser.

colli'na, Willd. = C. monta'na, L.

curt'a, ${ }^{5}$ Good. 69. p. 6. White Sedge.

v. alpic'ola ${ }^{6}$ (Wahl.)

Darallia'na, Sm. 1. p. 6. Tufted dixcious Sedge.7

depaupera'ta, Good. 3. p. 5, 6. Starved Wood Sedge.

digitata, L. 13. p. 5. Fingered Sedge.

1572

$\mathrm{x} .1637$

1125

1122

1112

x.1662

1141

dioic'a, L. 77. p. 5, 6. Creeping dicecious, or Separate-headed $S$. dist'ans, L. 49. p. 6. Distant-spiked Sedge. Loose Carex. dis'ticha, ${ }^{8}$ Huds. 78. p. 6. Soft Brown Sedge.

1576

$1576 \mathrm{~b}$

1554

1608

1592

1553

1612

1560 x. 1631

$\mathrm{x} .1632$

$\mathrm{x} .1611$

x. 1664

x. 1650

x. 1610

x.1668

x.1617 
Ca'rex, L. continued.

divi'sa, Huds. 26. p. 5, 6. Bracteated Marsh S. Divided Carex.

E.B.

x.1616

divul'sa, Good. 49. p. 5, 6. Gray Sedge. Carpeting S.

ech'ina'ta, ${ }^{1}$ Murr. 109. p. 5, 6. Little Prickly S. Star.headed C. v. Gryp'us (Schk.)

èlonga'ta, L. 15. p. 6. Elongated Sedge.

eri'ceto'rum, Poll. 3. p. 5, 6. Silvery Heath Sedge.

extens'a, Good. 48. p. 6. Long bracteated Sedge.

v. pu'mila, Anders.

f'liform'is, L. 34. p. 5. Slender-leaved Sedge.

fla'va, L. 58. p. 5, 6. Fellow Sedge.

v. min'or, Tuwns.

v. lep'idocarp'a (Tausch)

fri'gida, All. 1. p. 8. Narrow-fruited Alpine Sedge.

fulv'a, Good. 78. Tawny Sedge.

v. Hornschuchia'na, ${ }^{2}$ Bab. Short-spiked Sedge.

v. xanthocarp'a ${ }^{3}$ (Degl.)

r. speirostach'ya $=r$. Hornschuchia'na.

Gibso'ni, Bab. = C. Goodenow'ii, r. Gibso'ni.

glauc'a, Scop. 106. p. 6, 7. Glancous Heath Sedge.

v. Michelia'na, ${ }^{5}$ Syme.

v. stictocarp'a, ${ }^{8} \mathrm{D}$. Don.

Goodenow'ii, ${ }^{7}$ J. Gay. 106. p. 5, 6. Common Sedge.

v. juncell'a, Fr.

v. Gibsu'ni' ${ }^{8}$ (Bab.) p. 6. Gibson's Carex.

Graha'mi, Boott.=C. pull'a, v. Graha'mi.

hel'vola, Blytt. 1 .

hirt'a, L. 90. p. 5, 6. Hammer Sedge. Hairy Sedge.

r. ébract'ea ta, Syme.

hord'eiform'is, Wahl. p. 6. Barley Carex.

Hornschuchia'na, Hoppe = C. fulv'a, v. Hornschuchia'na.

hum'ilis, ${ }^{9}$ Leysser. 7. p. 5. Dwarf Silvery Sedge.

incurv'a, Lightf. 14. p. 6, 7. Curved Sedge.

intermed'ia, Good.= Ca'rex dis'ticha, Huds.

involu'ta, Bab. = C. rustra'ta, v. involu'ta.

irrig'ua, Hoppe. 13. p 6. Broad-leaved Nud Sedge.

1568

$\times .1625$

1569 (x.1826)

$1569 \mathrm{~b}$

1574

1597

1615

$1615 \mathrm{~b}$

1618

1616

$1616 \mathrm{~b}$

$1616 \mathrm{c}$

1604

1614

$1614 \mathrm{~b}$

$1614 \mathrm{c}$

158

$1588 \mathrm{~b}$

$1588 \mathrm{c}$

1587

$1587 \mathrm{~b}$

1587?

1573

1619

$1619 \mathrm{~b}$

x. 1630

x.1654

$\mathrm{x} .1675$

$\mathrm{x} .1676$

x.1672

x. 1673

x. 1669

(x.1670)

x. 1670

x. $16+4$

1143

x. 1645

x.1646

(x. 1643)

x.1684

$x .1677$

1134

1594

1558

x. 1651

$\mathrm{x} .1615$

1127

1120

x. 1681

1589

$\mathrm{x} .1648$

læviga'ta, Sm. 54. p. 6. Smooth-stalked beaked Sedge.

1610

1575

$\mathrm{x} .1666$

x. 1633

1109

1108

lèpori'na, " L." Benth.= C. ova'lis, Good.

lëpori'na, "L," H. and A. = C. lăgopi'na, Wabl.

limo'sa, L. 19.p.6. Narrow-leaved Mud S. Green\& Gold Carex. 1590

$\mathrm{x} .16+7$

1142

Miche'lia'na, Sm. = C. glauc'a,. . Miche'lia'na.

Mielichof'eri, $\mathrm{Sm}_{.}=$C. va'gina'ta, Tausch.

monta'na, ${ }^{11}$ L. 9. p. 4, 5. Monentain Sedge.

mu'rica'ta, L. 78. p. 5, 6. Greater Prickly Sedge.

v. pseud'o-divuls'a, Syme.

Oede'ri, Ehrb. 32. p. 7. Eder's Sedge.

or'nithop oda, Willd. 2. Bird's Foot Sedge.

ora'lis, ${ }^{12}$ Good. 110. p. 6. Oval-spiked Sedge.

v. bractea'ta, Syme.

pallesc'ens, L. 79. p. 6, 7. Pale Sedge.

paludo'sa, Grood. 70.p. 5, 6. Lesser Pond Sedge. Marsh C.

v. Ko'chia'na, Gaud.

pānic'ea, L. 111. p. 6,7. Pink-leaved Sedge. Carnation C.

pa'nicula'ta, I. 82. p. 6, 7. Greater panicled Sedge.

v. rig'ida, A. Blytt.

paradox'a, Willd. 4. p. 7. Paradoxical Sedge.

pauciflo'ra, Lightf. 26. p. 6, 7. Few-flowered Sedge.
1595

1567

$156 \% \mathrm{~b}$

1617

1593

157

$157 \% \mathrm{~b}$

1600

1621

$1621 \mathrm{~b}$

1601

1564

$1564 \mathrm{~b}$

1563

1557 $\times, 1652$

x. 1624

x.1674

x. $163 t$

x. 1657

$\mathrm{x}, 1678$

1135

1150

x. 1658

1140

$\mathrm{x} .1622$

1115

x. 1621

x. 1614
(1108)

1 Carex stellulata. 2 C. speirostachra.-C. fulva, v. speirostachya,-C. Hornschuehians. 3 C. xanthocarpa. 4 C. recurva. 5 C. Micheliana. 6 C. stictocarpa. 7 C. crespitosa, Good. - C. vulgaris. 8 C. Gibsoni. 9 Carex clandestina. 10 C. leparina, H. \& A. 11 C. collina. 12 C. leporina, Benth. 
Ca'rex, L. continued.

pend'ula, Huds. 72. p. 5, 6. Great Pendulous Sedge.

Persoon'ii, Sieb. = C. curt'a, v. alpic'ola.

phreostach'ya, Sm. = C. vagina'ta,'Tausch.

pilulif'era, L. 95. p. 6. Round or Pill-headed Sedge.

v. Lees'ii, ${ }^{1}$ Ridley.

præ'cox, Jacq. [vern'a, Chaix.] 94. p. 4,5. Vernal Sedge. Early Carex.

v. capita'ta, Ar. Benn.

Pseud'o-cype'rus, L. 46. p. 6. Cyperus-like Sedge.

pu'lica'ris, L. 104. p. 5, 6. Flea Sedge.

pull'a, Good. 11. p. 7. Russet Sedge.

v. Graha'mi 2 (Boott) Graham's Sedge.

puncta'ta, Gaud. 8. p. 6. Dotted-fruited Sedge.

ra'riflo'ra, Sm. 4. p. 6, 7. Loose-flowered Alpine Mud S. recurv'a, Huds. $=$ C. glauc'a, Scop.

remo'ta, L. 78. p. 6. Distant-spiked Sedge. Remote-flowered $C$. rig'ida, Good. 27. p. 6-8. Stiff Mountain Sedge. Rigid S.

v. in'feralpi'na, Lrestad.

ripa'ria, Curtis. 72. p. 5. Greater Pond or River Sedge.

rostra'ta, ${ }^{3}$ Stokes. 99. p. 6. Slender-beaked Bottle Sedge.

v. èla'tior, Blytt.

v. involu'ta ${ }^{4}$ (Bab.) 2. Involute-leaved Sedge.

rupest'ris, All. 5. p. 7. Rock Sedge.

[sali'na, Wahl.]

v. kattegatens'is (Fr.) 1.

saxa'tilis, I.

saxum'bra, F. A. Lees = C. pilulif'era, v. Lees'ii.

speirostach'ya, Sm. = C. fulv'a, v. Hornschuchia'na.

stellula'ta, Good. $=$ C. ech'ina'ta, Murr.

stictocarp'a, Sm. = C. glauc'a, v. stictocarp'a.

[leaved $C$. strict'a, ${ }^{5}$ Good. 40. p. 5, 6. Tufted S. Glaucous straightv. turfo'sa (Fr.)

strigo'sa, Huds. 35. p. 5, 6. Loose pendulous or Thin-spiked Sedge. sylva'tica, Huds. 83. p. 5, 6. Pendulous Wood Sedge.

ter'etiusc'ula, Good. 53. p. 6. Lesser panieled or Clustered S.

r. Ehrhart'ia'na (Hoppe)

to'mento'sa, L. 1. p. 6. Larger Downy-fruited Sedge.

trinerv'is, Degland. 1. p. 7, 8 .

ustula'ta Wahl. 1. p. 7. Seorched Alpine Sedge.

va'gina'ta, ${ }^{6}$ Tausch. 9. p. 7. Short Brown-spiked Sedge. ${ }^{7}$

Vahl'ii, Schk. = C. alpi'na, Sw.

ve'sica'ria, L. 69. p. 5, 6. Short-spiked Bladder Sedge. ${ }^{8}$

v. dich'roa (Anders.) Fr.

vi'tilis, Fr. = C. curt'a, v. alpic'ola.

vulga'ris, Fr. = C. Goodenow'ii, Gay.

vulpi'na, L. 83. p. 6. Great Sedge. Fox Carex. ${ }^{8}$ vul'pinö̈'des, Mich.

xanthocarp'a, Degl. = C. fulv'a, v. xanthocarp'a.

Carli'na, L. Compos'ıтe, Composites. Syn. PEq. vulga'ris, L. 77. b. 6-10. Carline Thistle.

Carpi'nus, L. Cu'PULIF'ER.e.

Monoec. Pol.

Be'tulus, L. 35. t. 5. Hornbeam.

Carricht'era, DC.

vell'æ, DC. $=$ Vell'a ann'ua, $\mathrm{L}$.

Car'um, L. Umbellif'ere, Umbellifers. Pent. Dig.

Bulbocas'tanum, ${ }^{10}$ Koch. 4. p. 6, 7. Great Earth-nut. ${ }^{11}$

Car'vi, L. b. 6, 7. Caraway.
L.C.

1606

1596

$1596 \mathrm{~b}$

1598

$1598 \mathrm{~b}$

1620

1555

1625

$1625 \mathrm{~b}$

1613

1591

1570

1584

$1584 \mathrm{~b}$

1622

1623

$1623 b$

$1623 \mathrm{c}$

1556

1586

$1586 \mathrm{~b}$

x. 1683, 4

$\mathrm{x} .1626$

$1581 \quad \mathrm{x} .1638$

$1581 \mathrm{~b}$

1607

1609

1562

$1562 \mathrm{~b}$

1599

1583

1603

1602

1624

$1624 \mathrm{~b}$

156

1566

79

793

1275

v. 698

$\mathrm{x} .1661$

$\mathrm{x} .1665$

x. 1619

x. 1620

$\mathrm{x} .1656$

x. 1663

x. 1659

x. 1682

1149

x. 1643

x. 1623

1116

633

iv. 583

\begin{tabular}{l}
632 iv. 582 \\
\hline
\end{tabular}

B.

1147

1131

1129

1146

1105

1139

1113

$(1148)$

1106

1121

1111

(1122)

1145

1144

1132

viii. 1293

911

411

410

3 Carex saxumbra. ${ }^{2}$ C. Grahami. ${ }^{3}$ C. ampullacea. 4 C. involuta. 5 C. caspitasa, Gay. 6 C. Mieli choferi.-C. phxostachys. 7 Loose-spiked Rock Sedge. 8 Large-fruted Bladder Sodge. 9 Great compound Prickly Sedge. 10 Bunium Bulbocastanum. I1 Tuberous Caraway. 
Car'um, I.-continued.

Hexuo'sum, Fr. = Co'nopod'ium de'nuda'tum, lioch.

Pet'roseli'num, Benth. b. 6-8. Parsley.

seg'etum, ${ }^{2}$ Benth. 44. a. or b. 8, 9. Corm Parsley. vert'icilla'tum, ${ }^{3}$ Koch. 20. p. 7. 8. Whorled Caraway.

CAR'YOPHTLL'Fe, Juss. Cloveworts.

Castan'ea, Tourn. Cu'Pulif'ere. Monnec. Pol.

sati'va, Mill. t. 5. Sweet Chestnut. Spanish Chestnut. vesc'a, Gærtn. $=$ C. sati'va, Mill.

rulga'ris, $\mathrm{L} .=$ C. sati'va, Mill.

Cătabro'sa, Beauv. Grämis'e.e, Grasses. Tri. Dig. aqua'tica, ${ }^{3}$ Beaur. 86. p. 5, 6. Water Whorl-grass or Hair-grass. ${ }^{6}$ v. littora'lis, Parn.

Cătapod'ium, Link. lölia'ceum, Link = Festu'ca lölia'cea, Huds.

Cauc'alis, L. UmbelliF'era, Umbellifers. Pent. Dig. Anthris'cus, ${ }^{7}$ Huds. 106. b. 7-9. Upright Hedge-Parsley. arvens'is, ${ }^{8}$ Huds. 57. b. 7-9. Field Hedge-Parsley. daucoïdes, L. 27. a. 7. Small Bur-Parsley. infest'a, Curt. $=$ C. arvens'is, Huds. la'tifol'ia, L. 7. a. 7. Great or Broad Bur-Parsley. nodo'sa, ${ }^{9}$ Scop. 71. b. 5-7. Knotted Hedge-Parsley. Celastrin'ex, Br. Spindle Trees.

Centaure'a, L. Compos'rte, Composites. Syn. Frus. asp'era. ${ }^{10}$ L. C. p. 7-9. Rough Star-thistle. Jersey S. Calcitra'pa, L. 16. b. 7-9. Star-thistle.

Cy'anus, L. 90. a. or b. Corn Blue-bottle. Corn-flower. Isnard'i, L. $=$ C. asp'era, L.

Ja'cea, L. p. 8, 9. Brown-rayed Knapweed.

ni'gra, L. 110. p. 6-9. Black discoid Knapweed.

v. decip'iens, ${ }^{11}$ (Thuill.) Black-rayed Knapweed.

nigresc'ens, Willd. $=$ C. ni'gra, v. decip'iens.

pa'nicula'ta, L. C. b. 7.

Seăbio'sa, L. 81. p. 7-9. Greater Knapweed. sol'stitia'lis, L. a. 7-9. St. Barnaby's Thistle. Yellow Star-thistle.

Calcitra'pa, Dufr. a. 6. Cut-leaved Valerian.

la'tifol'ius, Dufr. $=$ C. rub'er, DC. rub'er, ${ }^{12}$ IC. p. 6-9. Red Valerian.

Centun'culus, L. Privula'cese, Primworts. 'Tetran. Mon. min'imus, L. 62. a 6-10. Bastard Pimpernel. ${ }^{13}$

Cǒphalanthe'ra, Rich. Orcun'es, Orchids. Gyn. Mon. ensifol'ia, ${ }^{14}$ Rich. 34. p. 5, 6. Long-leaved Helleborine. ${ }^{15}$ grandiflo'ra, Bab. $=$ C. pall'ens, Rich.

pall'ens, ${ }^{18}$ Rich. 30. p. 5, 6. Large White Helleborine.

ru'bra, ${ }^{17}$ Rich. 4. p. 6, 7. Red Helleborine. Purple $H$.

Corast'ium, L. Car'Yop'yll'e, Cloveworts. Dec. Pent. alpi'num, L. 12. p. 6-8. Hairy Alpine Monse-ear Chickweed.

v. lana'tum ("Lam.")

v. pubesc'ens, Syme.

v. Edmonsto'ni, H. C. Wats.

aqua'ticum, I. = Stella'ria aqua'tica, Seop.

arct'icum, Lange? 1.

arvens'e, L. 69. p. 4-8. Field Mouse-ear Chickweed.

v. Andrew'ii, Syme.

a'trovir'ens, Bab. = C. tetrand'rum, Curt.
L.C.

630

$63 \mathrm{I}$

629

184

1278

(viii. 1290)

viii. 1290

1693

$1693 \mathrm{~b}$

$x i .1750$

1243

677

676

675

674

678

315

820

821

818

815

816

$816 \mathrm{~b}$

819

817

822

714

B.

407

408

409

iv. 581

ii. 191

(1)

iv. 620

445

(iv.619)

iv. 617

(446)

447

446

iv. $621 \quad 444$

ii. 317

v.710

572

573

v.71I

571

v. 705

v. 706

569

v. 707

v. 708

$5 \% 0$

v.712

574

iv. $66 j$

713

ir. 664

481

959

vii. 1149

659

1338

ix. 1484

982

ix. 1485

981

1339

1337

(ix. 1485)

(98I)

ix. $1+83$

217

ii. 223

983

$217 a$

$217 \mathrm{~b}$

217c

218

219

ii. 225

159 \begin{tabular}{l|l} 
iv. 619 & 446 \\
iv. 618 & 448
\end{tabular}

1 Petroselinum sativum. 2 P. segetum.-Sison segetum. 3 Sium rerticillatam.-Sison rertivillatnm. 4 Castanea vesca. - C. vulgaris.-Fagus Castanea. 5 Aira aquatica. 6 Water Catabrose. 7 Torilis Anthriscus. $8 \mathrm{~T}$. infesta.-C'ancalis infesta, 9 Torilis nodosa, 10 Centaurea Isnardi. 11 C. nigrescens. 12 Centranthus latifolins.- Valeriana rabra. 13 Small Chaffweed. 14 Epipactis ensifolia.-Serapias easifulia. 15 Narrowleaved White Helleborine. 16 Cephalanthera grandifura,-Epipactis granliflora.-Serapias grandiflora. 27 Epipactis rahra. - Serapias rubra. 
Cerast'ium, L. - continued. glom'era'tum, 'Thuill. v. apet'alum, Dum.

110. a. 4-9. Broad-leaved M. C. ${ }^{2}$

L.C. E B

215 ii.221

$215 \mathrm{~b}$

la'tifol'ium, Sm. 11. p. 5. Broad-leaved Alpine Chickweed. pedunc'ula'tum, Bab. = C. tetrand'rum, Curt.

pu'milum, Curt. 8. a. 5. Curtis' Monse-ear Chickwced.

quāternell'um, ${ }^{3}$ Fenzl. 50. a. 5, 6. Upright Monchia, or $C$. se'midecand'rum, L. 83. a. 3-5. Lesser Mouse-ear Chickweed. tetrand'rum, 'Curt. 67. a. 4-10. Dark-green or Four-eleft M. C. trig'ynum, ${ }^{5}$ Vill. 5. p. 7, 8. Three-styled Alpine Chickweed. ${ }^{6}$ trivia'le, ${ }^{7}$ Link. 111. p. 4-8. Narrow-leaved Mouse-ear $C$.

v. holost'eoi'des, Fr.

v. pentand'rum, Syme.

v. alpest're, "Lindbl."

visco'sum, L. = C. trivia'le, Link.

vulga'tum, L. = C. glom'era'tum, Thuill.

Cer'asus, Tourn.

auste'ra, Leight. = Pru'nus Cer'asus, $\mathrm{L}$.

av'ium, Mœnch = Pru'nus av'ium, L.

Pad'us, DC. = Pru'nus Pad'us, L.

Cer'atophyl' exe, Gray. Hornworts.

Cer'atophyll'um, L. Agg. 56. Cer'atophrld'ex, Hornworts, Monœe. Pol.

aqua'ticum, Wats.

demers'um, L. p. 7-9. Hornwort. Spring-fruited $H$.

v. ox'yacanth'um, Cham.

submers'um, L. p. 9. Unarmed Hornwort.

Cet'erach, Willd. Fin'rces, Ferns. Crypt. Fil. offic'ina'rum, ${ }^{8}$ Willd. 65.p. 7-9. Sealy Spleenwort. ${ }^{9}$

v. crena'tum, Milde.

Chær'ophyll'um, L. UмBellif'eræ, Umbellifers. Pent. Dig. Anthrisc'us, Lam. $=$ Anthrisc'us vulga'ris, Pers.

ar'oma'ticum, ${ }^{10}$ L. p. 6. Broad-leaved Chervil. Aromatic Cou'

Parsley.

aur'eum, ${ }^{11}$ L. p. 6. Tawny-fruited Chervil.

sati'vum. Lam. = Anthrisc'us Cerefol'ium, Hoffm.

sylvest're, $L_{\text {. }}=$ Anthrisc'us sylvest'ris, Hoffm.

te'mulent'um, Sm. = C. te'mulum, L.

te'mulum, ${ }^{12}$ L. 97. p. 6, 7. Rough Chervil.

Chamagro'stis, Borkh.

min'ima, Borkh. = Mibo'ra vern'a, Adans.

Char'a, L. Chara'cex, Charads. Crypt. Char.

alo' pecu'roï'dea, "Del." =Lamprothamn'us alo'pecu'roï'des, A.Br.

aspera, Willd. 27. p. 7, 8. Rough Water Horsctail.

v. capilla'ta, A. Br.

v. cur'ta, A. Br.

v. sub'inerm'is, Kuetz.

v. lacust'ris, H. \& J. G.

balt'ica, Bruzel. 2.

v. affi'nis, ${ }^{13}$ H. \& J. G.

Borre'ri, Bab. = Tolypell'a prolif'era, Leonh.

Braun'ii, Gmel. 1. a? 8, 9. Braun's Chara.

canesc'ens, ${ }^{14}$ Loisel. 2. a. 8. Bearded Chara.

conni'vens, ${ }^{15} \mathrm{~A}$. Br. 2.

contrária, ${ }^{16}$ Kuetz. 20.

v. hispid'ula, $\mathrm{A}$. Br. 1 Cerastinm rulgatnm. 2 Clustered Mouse-ear Chickweed. 3 Moenchia erecta.-M. glanca.-Sagina
4 Cerastium atrovirens.-C. pednnculatum. - Sagina cerastoides. 5 Stellaria cerastoides. 6 Stitchwort Chichueed.-Alpine Staniort. 7 Cerastium viscosum. 8 Asplenium Ceterach.-Grammitis Ceterach.Scolopendium Ceterach. 9 Common Ceterach. 10 Myrrhis aromatica. 11 M. aurea. 12 Chærophyllum temulentum. - Myrrhis temulenta. 13 Chara hispida v. baltica. 14 C. crinita. 15 C. fragilis, v, connivens. 16 C. fœida, v, contraris. 
Char'a, L.-continued.

crini'ta Wallr. = C. canesc'ens, Loisel.

flex'ilis, L. = Nitell'a Hex'ilis, Ag.

fœ'tida, Br. = Char'a vulga'ris, $\mathrm{L}$.

v. contra'ria, Coss. \& Germ. = C. contra'ria, Kuetz.

fragif'era, Durieu. 1. p. 7, 8. Strawberry Chura.

frag'ilis, Desv. 72. p. 6-8. Fragile Chara.

v. barba'ta, Gant.

v. capilla'cea, Coss. \& G.

v. Hedwig' ii, ${ }^{1}$ Iruet $z$.

v. delica'tula, $A$. Br.

v. Sturrock'ii, H. \& J. G.

v. conni'vens $=$ C. conni'vens, A. Br.

glom'era'ta, Desv. = Tol'ypell'a glom'era'ta, Leonh.

grac'ilis, Sm. = Nitell'a grac'ilis, Agardh.

Hedwig'ii = C. frag'ilis, v. Hedwig'ii.

hisp'ida, L. 36. p. 7.9. Prickly W. H. Bristly Chara.

v. ma'cracanth'a, A. Br.

v. gymnot'eles, A. Br.

v. rud'is, A, Br.

v. horr'ida, A. Br.

v. balt'ica, Hartm. =C. balt'ica, v. affi'nis.

v. pseud'o-erini'ta, A. Br. = C. pol'yacanth'a, A. Br.

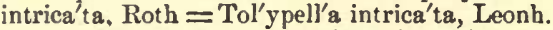

mu'crona'ta, A. Br. = Nitell'a mu'crona'ta, Kuetz.

nidif'ica, sm. = Tol'ypell'a glom'era'ta, Leonh.

papillo'sa, Kuetz. 1 .

pol'yacanth'a, ${ }^{2}$ A. Br. 11.

polysperm'a, A. Br. = Tol'y pell'a intrica'ta, Leouh.

prolif'era, A. Br. = Tol'ypell'a prolif'era, Leonb.

Smith'ii, Bab. ='Jol'y pell'a glom'era'ta, Leonh.

stellig'era, Bauer = Lychn'othamn'us stell'iger, A. Br.

sy̆ncarp'a, Thuill. = Nitell'a opa'ca, Ag. and N. capita'ta, Ag. tenuiss'ima, Desv. = Nitell'a tenuiss'ima, Kuetz.

to'mento'sa, L. 1. p. Tomentose Chara.

translu'cens, Pers. = Nitell'a translu'cens. Ag.

vulga'ris, ${ }^{3}$ L. 65. a. 6-8. Fetid Chara or Water Horsetail.

v. long'ibract'ea'ta. Kuetz.

v. papilla'ta, Wallr.

v. a'trovir'ens (Lowe).

v. mel'anopyre'na, $\mathrm{A} . \mathrm{Br}$.

sub C. fœ'tida.

v. erassicau'lis, Kuetz.

L.C.

1834

1833

$1833 \mathrm{~b}$

$1833 \mathrm{c}$

$1833 d$

$1833 \mathrm{e}$

$1833 \mathrm{f}$

1842

$1842 \mathrm{~b}$

$1842 \mathrm{c}$

$1842 \mathrm{~d}$

$18+2 \mathrm{e}$

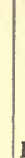

1840

1837

(xii.1918)

xii.1918

xii.1910

1841

(xii.19]3)

1843 (xii.1914)

$1843 \mathrm{~b}$

$1843 \mathrm{c}$

$1843 \mathrm{~d}$

$1843 e$

$1843 \mathrm{f}$

183.3

xii.1899

Cheir'i, L. p. 5, 6. Wall-flower. Gilliflower.

frut'iculo'sus, L. $=$ C. Cheir'i, L.

inca'nus, $\mathrm{L}$. = Math'iola inca'na, R. Br.

sinua'tus, L. = Math'iola sinua'ta, R. Br.

Chel'ídon'ium, L. Papa'vera'ces, Poppyworts. Polyan. Mon.

Glauc'ium, L. = Glauc'ium fla'vum, Crantz.

hyb'ridum, Loiss. = Røme'ria hyb'rida, DC.

lac'inia'tum, Mill. = C. ma'jus, "v. lac'inia'tum.

ma'jus, L. 96. p. 5-8. Greater Celandine. Swallow-wort.

v. lac'inia'tum ${ }^{5}$ (Mill.)

CuE' Norod'1a'CEA, Juss. Chenopods.

Che'nopod'ium, L. Cirf' Nopod'ia'Ce.s, Chenopods. Pent. Dig. acu'tifol'ium, Sm. = C. polysperm'um, v. spica'tum.

alb'um, L. 109. a. 7-9. White Goosefoot. Fat $H_{\text {ch. }}$

1 C. Hedwigii. 2 Chara hispida, v. psendo-crinita. $3 \mathrm{C}$. fotida. 4 Cheiranthus fraticnlosus. 5 Cheli-

B.

xii.1914

xii.1915

xii.1920

xii.1921

xii.1916

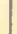

E.13. 
Che'nopod'ium, L.-continued.

r. cand'icans (Lam.) [inca'num, Moq.]

v. vir'ide (L.) Green Goosefoot.

v. paga'num (Reichb.) [viridesc'ens, St. Amans]

Bou'us-Hen'ricus, L. 97. p. 5-8. All-good. Wild Spinach. ${ }^{1}$ botryo'des, Sm. 6. a. 8,9. Many-clustered Goosefoot. fi'cifol'ium, Sm. 18. a. 8, 9. Fig-leaved Goosefoot. frŭtico'sum, L. = Suse'da frŭticı'sa, Forsk.

glauc'um, L. 12. a. 8,9. Oak-leaved $G$. Glaneous $G$. hyb'ridum, L. 23. a. 8, 9. Maple-leaved Goosefoot. intermed'ium, M. \& K. = C, urb'icum, v. intermed'ium. marit'imum, L. = Suæ'da marit'ima, Dum.

mura'le, I. 40. a. 8, 9. Nettle-leaved Goosefoot. ol'idum, Curt. = C. Vulva'ria, L.

op'ulifol'izm, Schrad.

polysperm'um, L. 48. a. 8-10. Many-sceded Goosefoot. ${ }^{2}$

v. spica'tum, ${ }^{3}$ Koch.

v. cymo'sum, Koch.

v. acu'tifol'ium $=$ v. spica'tum.

ru'brum, I. 64. a. 8, 9. Red Goosefoot.

v. pseud'o-botryo'des, H. C. Wats.

urb'icum, L. 38. a. 9, 10. Upright Goosefoot.

v. deltoï'deum (Lam.)

r. intermed'ium (Mert. \& Koch)

Vulva'ria, ${ }^{5}$ L. 37. a. 8-10. Stinking Goosefoot.

Cherle'ria, $\mathbf{L}$.

sed'oî'des, $L .=A r^{\prime}$ ēna'ria sed'oin'des, Scbultz.

Chirōni'a, L.

littora lis, Sm. = Er'ythræ'a littora'lis, Fr.

Centaur'ium, Curt. = Er'ythræ'a Centaur'ium, Pers. pulchell'a, Swartz = Er'ythræ'a pulchell'a, Fr.

Chlo'ra, L.

per'folia'ta, L. = Blucksto'nia per'folia'ta, Huds.

Chrysanth'emum, L. Compos'its, Composites. Syn. Sup.

Cham'omill'a, E. Mey. = Ma'trica'ria Cham'omill'a, L.

in'odo'rum, C. H. Schultz $=$ Ma'trica'ria in'odo'ra, I.

v. marit'imum, Yers. $=\mathbf{M}$. in'odo'ra, v. marit'ima.

Leucanth'emum, L. 108. p. 6-8. Great White Ox-eye. ${ }^{6}$

marit'inum, Sm. = Ma'trica'ria in'odo'ra. v. marit'ima.

Parthen'ium, ${ }^{7}$ Pers. p. 7.9. Common Feverfew.

seg'etum, L. 109. a. 6-9. Corn Marigold. Yellow Ox-eye.

'Tan'ace'tum, syme = 'Tan'ace'tum vulga're, L.

Chrysoc'oma, Cass.

Linos'yris, L. = Ast'er Linos'yris, Bernh.

Chry'sosple'nium, L. SAxifraG'Ex, Saxifrages. Dec. Dig. altern'iful'ium, L. 67. p. 4-6. Alternate-leaved Golden Saxifrage. oppos'itifol'ium, L. 104. p. 5-7. Opposite-leaved Golden S.

Cicen'dia, Adans. Gentin'xex. Gentianwerts. Tetran. Mon. Candoll'ii, Griseb. = C. pusill'a, Griseb.

fi'liform' is, Del.= Mi'crocal'a fi'liform'is, Link. pusill'a, ${ }^{3}$ Griseb. C. a. 6, 7. Least Cicendia.

Cicho:'ium, L. Compos'rte, Composites. Syn. Feq. Int'ybus, L. 63. p. 7-10. Wild Succory. Chicory." Eudive.

Cicu'ta, L. Unimelif'er.e, Umbellifers. Pent. Dig. viro'sa, L. 32. p. 7, 8. Water Hemlock. Cowbane.

Cin'era'ria, $\mathrm{L}$. campest'ris, Retz. = Senec'io campest'ris, DC. palust'ris, L. = Senec'io palust'ris, DC.

L.C. E.B.

1181 a viii. 1188

$1181 \mathrm{~b}$ viii, 1189

$1181 \mathrm{c}$ viii. 1190

1190 viii. 1199

1188 viii. 1195

1183 viii. 1191

1189 viii. 1198

1185 viii. 1193

843

$8+7$

1184 viii. 1192

846

1182

$11: 9$ viii.1185, 6

1179 a (viü.1186)

$1179 \mathrm{~b}$

1187

viii. 1186

$1187 \mathrm{~b}$ viii. 1197

1186 viii. 1194

$1186 a$

$1186 \mathrm{~b}$

1180

viii. 1187

840

148

vi. 913

679

v. 719

v.717

v. .18

v.714

766

v.715

767

$r, 713$

v.:16

5.1

554

iv. 564

387

553 iv.563

356

vi.912

671

vi.911

672

823

v.7\$6

606

627

iv.571

400

1 Good King Henry.-Mercury Goosefoot.-Perennial Goosefoot. 2 Round-leared Goosefoot. -All-seed.Blite. 3 Chenopodium acutifolium, - C. polyspermnm, v, acutifolinm. 4 C. intermedium, $5 \mathrm{C}$. olidum. 6 Moon Daisy. 7 Matricaria Partherium.-Pyrethrum Parthenium. 8 Cicendia Caudollii. 
Circæ'a, L. ON'Agrar'iæ. Onagrads. Dian. Mon. alpi'na, L. 27. p. 7, 8. Alpine Enchanter's Nightshade. v. intermed'ia ${ }^{1}$ (Ehrh.) 30.

intermed'ia, Ebrh. = C. alpi'na, $\mathbf{r}$. intermed'ia.

Lutet'ia'na, L. 97. p. 6-8. Enchanter's Nightshade. v. intermed'ia, Lond. Cat.

Cistix'ex, Juss. Rock-roses.

Cist'us, Tourn.

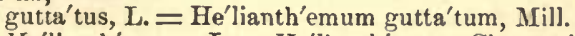

He'lianth'emum, L. = He'lianth'emum Chamæeist'us, Mill.

marifol'ius, $\mathrm{L} .=\mathrm{He}^{\prime}$ lianth'emum marifol'ium, Mill.

pol'ifol'ius, L. $=\mathrm{He}^{\prime}$ lianth'emum pol'ifol'ium, Mill.

to'mento'sus, Scop. = He'liantlı'emum Chamæcist'us, Mill.

Clad'ium, 1'. Br. CYr'era'ces, Sedges. Dian. Mon. germa'nicum, ${ }^{2}$ Schrarl. 37. p.7,8. Fen Sedge. Prickly Bog Rush ${ }^{3}$ Marisc'us, R. Br. = C. germa'nicum, Schrad.

Clayto'nia, L. Portula'ce. Purslanes. Pent. Mon. alsi'noï'des, Sims. a. 5-7. Chickweed-leaved Claytonia. per'folia'ta, Donn. a. 5-7. Perfoliate Claytonia.

Cle'matis, L. Raxunc'ula'ces, Crowfoots. Polyan. Pol. Vitalb'a, I. 47. s. 7, 8. Traveller's Joy. Clematis.

Cli'nopod'ium, L.

vulga're, L. = Cal'aminth'a Cli'nopod'ium, Bentl.

Cni'cus, I. Coupos'rтx, Composites. Syn. Æq.

acau'lis, ${ }^{5}$ Willd. 42. p. 7-9. Dwarf Plume Thistle. arvens'is, ${ }^{6}$ Hoffm. 112. p. 7-9. Creeping Plume Thistle. Field T.

v. horr'idus (W.\& G.).

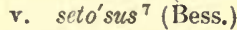

er'ioph'orus, ${ }^{8}$ Roth. 46. b. 7-9. Woolly-headed Plume Thistle. het'erophyll'us, ${ }^{9}$ Willd. 51. p. 7-9. Melancholy Thistle. ${ }^{20}$ hyb'ridi :

a. Gibso'ni, Wats.

b. Forste'ri," $\mathrm{Sm} .=$ palust'ris $\times$ prateus'is.

c. Woodward'ii, ${ }^{12}$ Wats. $=$ pratens' is $\times$ acau'lis.

d. Clark'ei, ${ }^{23}$ Wats. (=dub'ius, Willd. ?)=acau'lis $\times$ arrens' is.

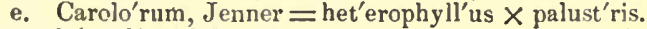

lanceola'tus, ${ }^{14}$ Willd. . 112. b. or a. 7-10. Spear Thistlc.

blera'ceus, I'.

palust'ris, ${ }^{25}$ Willd. 112. b. or a. Marsh Thistle.

pratens'is, ${ }^{26}$ Willd. 47. p. 6-8. Meadow Plume Thistle. seto'sus, Bess. $=$ C. arvens'is, v. seto'sus.

tubero'sus, ${ }^{17}$ Roth. 2. p. 8, 9. Tuberous Plume Thistle.

Cnid'ium, Cusson.

Sil'ăus, Spr. = Sil'ăıs pratens'is, Bess.

Cochlea'ria, L. CrucrF'Er.f, Crucifers. Tetrad. Siliq.

alpi'na, H. C. Wats. = C. offic'îna'lis, v. alpi'na.

an'glica, L. 43. p. or b. 5-7. Long-leaved English Scurvy-grass.

v. Hort'ii, Syme.

Armorac'ia, ${ }^{13}$ L. p. 5. Horse Radish.

da'nica, L. 43. a.5-8. Danish $S$. Hastate-leaved $S$.

grœnland'ica, Sm. = C. offic'inna'lis, v. alpi'na.

offic'ina'lis, L. 79. b. or p. 5-8. Common Scurvy-grass.

v. littora'lis, H. C. Wats.

v. alpi'na ${ }^{19}$ (Wats.) Bab. Mountain Seurvy-grass. polymorph'a, Syme.

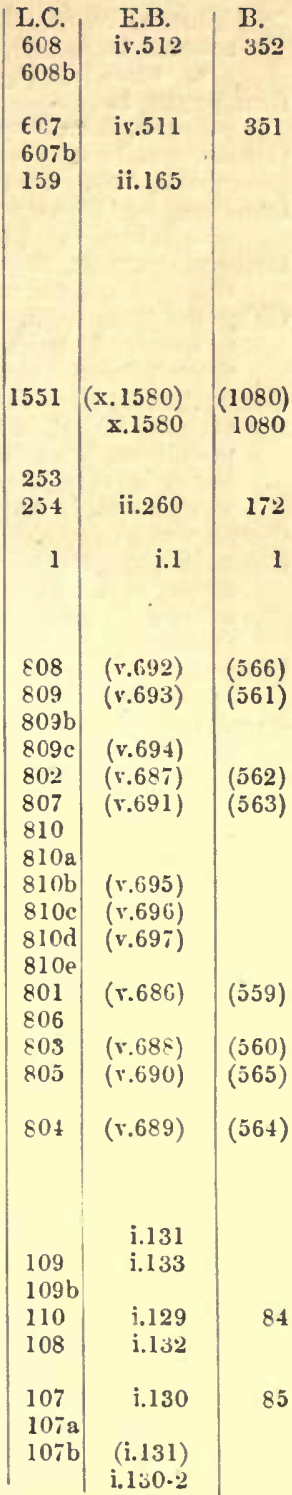

i. $130 \cdot 2$

1 Circea intermedia. 2 Cladium Marisens,-Schœnus Mariscus. 3 Tuig-rush. 4 Old Man's Beard. 5 Carduns acaulis. 6 C. arvensis. 7 Cnicus selosus.-Carduas arrensis, v, setosus. 8 C. eriophorus. 9 C. heterophyllus. 10 Dark Plume Thistle. 11 Carduus pratensi-palustris, 12 C. acauli-pratensis. 13 C. arvensi-acaulis. $14 \mathrm{C}$. lanceolatus. $15 \mathrm{C}$. palustris, $16 \mathrm{C}$. pratensis, $17 \mathrm{C}$. Iuberosus, 18 Armoracia rusticana. 19 Cochlearia alpina,-C. grenlandica. 
Colch'icum, Tourn. LI'LIA'ce.e, Lilyworts. Hex. Trig. autumna'le, L. 40. p. 8-10. Meadow Saffron. v. verna'le. (Spring-flowering form.)

Com'arum, L. palust're, L. = Potentill'a Com'arum, Nestl.

Compos'1t.

Coxif'erse, Juss. Conifers.

Coni'um, L. Umbellif'ere, Umbellifers. Pent. Dig. macula'tum, L. 101. b. 6,7. Common Hemlock.

Cond'ylocarp'us, Hoffm. offic'ina'lis, Koch. = 'Tordyl'ium offic'inna'le, L.

Co'nopod'ium, Kíoch. Umbellif'Er.e, Umbellifers. Pent. Dig. de'nuda'tum, ${ }^{1}$ Koch. 104. p. 5, 6. Earthnut. Tuberous Bunium. ${ }^{2}$ flexuo'sum = C. de'nuda'tum, Koch.

Convalla'ria, L. Li'Lja'Ces, Lilyworts. Hex. Mon. maja'lis, L. 56. p. 5, 6. Lily of the Valley. multiflo'ra, L. = Pol'ygōna'tum multiflo'rum, All. Pol'ygōna'tum, I. = Pol'ygōna'tum offic'īna'le, All.

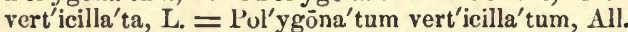
Coxtolv'ula'Ce. Juss. Bindweeds.

Convolv'ulus, L. Convolv'ula'ce.e, Bindweeds. Pent. Mon. arvens'is. L. 92. p. 6-9. Small Bindweed. se'pium, L. = Calysteg'ia se'pium, R. Br.

Sol'danell'a, L. = Calysteg'ia Sol'danell'a, R. Br.

Cony'za, L. squarro'sa, L. = In'ula Cony'za, DC.

Corall'orhi'za, Haller. OnChid'es, Orchids. Gyn. Mon. inna'ta, ${ }^{3}$ R. B1:. 15. p. 7, 8. Common Spurless Coralroot.

Cor'iand'rum, L. Umbellif'ere, Umbellifers. Pent. Dig. sati'vum, I. a. 6. Common Coriander.

CORNA'CE.E, DC. Cornels.

Corn'us, L. Conva'Ces, Cornels. Tetran. Mon. sanguin'ea, L. 66. s. 6,7. Dogwood. Prickwood. Wild Cornel." sue'cica, L. 14. p. 7, 8. Dwarf Cornel.

Cor'onill'a, L. Legu'uixo's. E, Legnminous Plants. Diad. Dec. var'ia, L. Rosy-flowered Crown Vetch.

L.C.

Coro'nopūs, Grert.

Ruell'ii, Grert. = Senebier'a Coro'nnpūs, Puir.

did'yma, Sm. = Senebier'a did'yma, Pers.

Corrig'iola, L. Ilz'ECeßrA'Ce littora'lis, L. 2, a. 7-9. Sand Strap-wort.

Coryd'alis, DC. Fuma'rin'Ce. Fumeworts. Diad. Hex. bulbo'sa, ${ }^{5}$ DC. p. 4, 5. Solid Bulbons Fumitory. ${ }^{6}$

cla'vicula'ta, ${ }^{7}$ I)C. 83. a. 6-8. White Climbing Fumitory. lu'tea, ${ }^{8}$ DC. p. 5-8. Yellow Fumitory or Corydalis. sol'ida, Hook, = C. bulbo'sa, DC.

Cor'ylus, Tolirn. Cu'pulif'er.e. Monœc. Pol. Aveila'na, I. t. 2, 3. Hazel. Colnut. Filbert.

Cor'yne'phorus, Beauv. Grämis'e.z, Grasses. Tri. Dig. canes cens, ${ }^{9}$ Beauv. 4. p. 7. Grey Aira. Grey Hair-Grass.

Coto'neast'er, Medic. Ros'ace.e, Roseworts. Icos. Pent. integer'rima, Medic. See vulgaris, Lindl.

vnlga'ris, ${ }^{10}$ Lindl. [integer'rima, Medic.] 1.s.5,6. Com. Cotoneaster. ${ }^{11}$

Cot'yle'don, L. Crassula'ce.e, Houseleeks. Dec. Pent. Umbili'cus, ${ }^{12}$ L. 53. p. 6, 7. Navelwort. Wall Pennywort.

Cramb'e, L. Crucir'er.e, Cruteifers. Tetrad. Silic. marit'ima, L. 32. p. 6-8. Sea-Kale.

Crassula'ce. DC, Honseleeks. 1 Conopodium flexuosum. - Bunium flexuosum.-Carum flexnosum. 2 Kipper-nut. - Pig-nut. 3 Ophrys
Corallorhiza. 4 Dogberry. 5 Corydalis solida. - Fumaria solida. 6 Tuberous-rooted Fumitory. 7 F. elaviculata. $8 \mathrm{~F}$. lutea. 9 Aira canescens. 10 Mespilus Cotoneaster. 11 Dwarf Quince-leared Medlur. 12 Umbilicus pendulinus.
1421

726

1318

617

640

1395

ri.923

vi. 923

vi. 924

vi. 925

1328

649

680

681

680

378

E.B.

B.

1054

ix. 1545

v. 680

iii. 1380

iv. 629

450

440

1028

68.3

684

685

978

453

iv. 635

iv. 634

457

456

831

(i.68)

i. 70

i. 69

i. 68

1276 viii. 1292

912

\begin{tabular}{l|l}
1672 & $\times 1.1729$
\end{tabular}

(1187)

535 iii.477

338

561

ir.539

358

152

i. 80

111

6


Cratæ'gus, L. Rosa'ce.e, Roseworts. Icos. Pent.

monog'yna, Jacq. = C. Ox'yacanth'a, v. monog'yna.

Ox'yacanth'a, I. 110. t. or s. 5, 6. Hawthorn. May.

v. ox'yacanth'oi'des ' (Thuill.) Glabrous White Thorn.

v. lac'inia'ta, Wallr.

v. kyrtosty'la (Fingerb.)

v. monog'yna ${ }^{2}$ (Jacq.) Common White Thorn.

ox yacanth'oï des, 'Thuill.=C. Ox'yacanth'a, v. ox'yacanth'oï'des.

tormina'lis, L. = Pyr'us tormina'lis, Ehrh.

Cre'pis, L. Compos'rтe, Composites. Syn. Alq.

bienn'is, I. 21. b. 6, 7. Large Rough Hawk's-beard.

fo'tida, ${ }^{3}$ L. 15. a. or b. 6, 7. Stinking Hawk's-beard.

hiera'cioi'des, ${ }^{4}$ W. \& K. 15. p. 7, 8. Scabious or Succory-lcavd $H$. $n i^{\prime}$ ccens'is, Balb.

paludo'sa, Moench. 60. p. 7-9. Marsh Hawh's-beard.

pulch'ra, ${ }^{6}$ L. a. 6-9. Small-flowered Hawk's-beard.

seto'sa, Hall. fil. b. 8. Bristly Hawk's-beard.

succi'sæfol'ia. Tausch = C. hiera'cioí'des, W \& K.

tarax'acifol'ia, ${ }^{7}$ Thuill. 23. b. 6, 7. Small Rough $H$. Beakcd $H$.

tecto'rum, Sm. $=$ C. vir'ens, L.

vir'ens, ${ }^{8}$ L. 106. a. 6-9. Sinooth Hawk's-bcard.

Cri'thmum, L. Umbellif'ere, Umbellifers. Pent. Dig. marit'imum, L. 25. p. 6-8. Rock, or Sea Samphire.

Croc'us, 'Tourn. Irio'ex, Irids. Tri. Mon.

argent'eus, Sal. p. 3.

aur'eus, Sibth. p. 2. Golden Crocus.

autumna'lis, Sm. $=$ C. sati'vus, $\mathbf{L}$.

biflo'rus, ${ }^{9}$ Mill. p. 2. Scotch Crocus.

min'imus, $\mathrm{H} \& \mathrm{~A} .=\mathrm{C}$. biflo'rus, Mill.

nu'diflo'rus, ${ }^{10}$ Sm. 8. p. 9, 10. Naked-flowering Crocus.

pra'cox, Haw. $=$ C. biflo'rus, Mill.

sati'vus," L. p. 9. Saffron Crocus.

spec'io'sus, Nils. = C. nu'diflo'rus, Sm.

vern'us, All. p. 4, 5. Purple Spring Crocus.

Crucif'ere, Juss, Crucifers.

Crypt'ogramm'a, R. Br. Frc'ices, Ferns. Crypt. Fil. crispa, ${ }^{12}$ R. Br. 54. p. 6, 7. Parsley Fern. Curled Rock Brakes.

Cucu'balus, Gærtn. CAR' rophrla'ex, Cloveworts. Dec. Trig. bacc'ifer, ${ }^{13}$ L. p. 8. Berry-bearing Chickweed.

baccif'erus, $\mathrm{L} .=\mathrm{C}$. bacc'ifer, $\mathrm{L}$.

Beh'en, L. = Sile'ne Cucu'balus, Wibel.

Oti'tes, L. = Sile'ne Oti'tes, Sm.

Cucurb'iti'ce.e, Juss. Cucurbits.

Cu'PCLIF'ER.e.

Cusc'uta, Tourn. Coxrolrcla'ces, Bindwceds. Pent. Dig. approxima'ta, Bab. a. 8, 9.

Epil'inum, Weihe. a. 7, 8. Flax Dodder.

Epithym'um, Murr. 43. a. 7-10. Lesser Dodder.

europa'a, L. 31. a. 7-9. Greater Dodder.

hassia'ca, Pfeift. a. 8, 9.

Trifol'ii, Bab. a. 7-9. Clover Dodder.

Cyath'ea, Sm.

frag'ilis, Sm. = Cystopt'eris frag'ilis, Bernh.

inci'sa, Sm. = Cystop teris alpi'na, Desv.

Cy'clamen, Tourn. Pri'sclaces, Primworts. Pent. Mon. europæ'um, Sm. = C. hed'eræfol'ium, Aiton.

hedercefol'ium, ${ }^{14}$ Aiton. p. 9. Ivy-leav'd i'yclamen. Sow-bread.

v. fica'riæfol'ium, Syme.

\begin{tabular}{l|} 
L.C. \\
534 \\
$534 \mathrm{a}$ \\
$534 \mathrm{~b}$ \\
$534 \mathrm{c}$
\end{tabular}

$534 \mathrm{~d}$

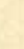

\section{8}

828

832

835

830

829

831

650

E. B.

iii. 480

iii.479-80

B.

$4 a$ (iii.479)

(iii.480)

iii.479

33

จ.819

596

v. 815

594

(v.820)

597

v.821

598

r. 817

v. 820

v. 816

593

r.818

595

iv.606

430

1374

ix. 1498

1373

ix. 1497

1376

ix. 1500

1018

1375

ix. 1499

1017

73

i. 79

1766

xii. 1844

$(12 \% 6)$

203

(ii.198)

ii. 198

609

iv. 517

1271

(viii.1288)

1011

vi.926

687

1013

vi.928

1012

vi. 927

688

1014

vi. 929

686

1 Mespilus oxyacanths. - Cratægus oxyacauthoides. 2 C. monogyna. 3 Borkhnusia fotida. I Crepis succisafolia.-Hieracium molle. 5 H. paludosum. 6 Prenanthes hieracifolia. 7 Borkbausia taraxacifolia.

Crepis tectorum. 9 Crocus uinimus.-C. pracnx. 10 C. speciosus. 11 C. antumnalis. 12 Allosorus crispus. - Pteris crispa. 33 Cucubalus bacuiferus. 14 Cyelemen earopaum 
Cyn'odon, Rich.

Dact'ylon, Pers. = Fibich'ia umbella'ta, Kinel.

Cyn'oglo'ssum, Tourn. Borĩañ'es, Borageworts. Pent. Alon. germa'nicum, Jacq. See monta'num, Lam.

monta'num, ${ }^{1}$ Lam. [germa'nicum, Jacq.] 17. b. 5-7. Greenleaved Hound's Tongue.

offic'inna'le, L. 75. b. or p. 6, 7. C. Hound's Tongue. Gipsy-flower. v. subgla'brum, Syme.

sylva'ticum, Haenke. $=$ C. monta'num, Lam.

Cyn'osu'rus, L. Grāmin'e.e, Grasses. Tri. Dig. crista'tus, L. 112. p. 7, 8. Crested Dog's-tail-grass. ech'ina'tus, L. C. a. 7. Rough Dog's-tail-grass.

Crpera'ce.e, Juss. Sedges.

Cype'rus, L. CyPera'ces, Sedges. Tri. Mon. fusc'us, L. 2. a. 8, 9. Brown Cyperus.

long'us, L. 7. p. 8, 9. Sweet C. English Galingale.

Cyp'riped'ium, L. Orchin'Es, Orchids. Gyn. Dian. Calc'eolus, L. 4. p. 5. Lady's slipper. Cypripede.

Cyst'ea, Sm.

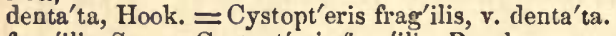
frag'ilis, Sm. $=$ Cystopt'eris frag'ilis, Bernh.

Cystopt'eris, Bernh. F'ir'ices, F'erns. Crypt. Fil. alpi'na, ${ }^{2}$ Desv. 1. p. 7. Alpine or Laciniate Bladder-fern. v. Dickiea'na, ${ }^{3}$ Milde.

angusta'ta, Sm. Narrow-fronted Bladder-fern.

denta'ta, Hook. = C. frag'ilis, v. denta'ta.

Dickiea'na, Sm. = C. alpi'na, v. Dickiea'na.

frag'ilis, ${ }^{4}$ Bernh. 79. p. 7, 8. Brittle Bladder-fern. v. denta'ta, ${ }^{5}$ Hook.

monta'na, Bernh. 4. p. 7, 8. Mountain Bladder-fcrn. re'gia, Presl. = C. alpi'na, Desv.

Cyt'isus, L. Leaumro'se, Leguminons Plants. Diad. Dec. scopa'rius, ${ }^{8}$ Link. 109. s. 5, 6. Common Broom. v. prostra'tus, C. Bailey.

Dabo'cia, D.Don. ER' ICA' $^{\prime} \mathrm{CE}$, Heathworts. Oct. Mon. pol' ifol'ia, ${ }^{7}$ Don. I. s. 8. Irish Meuziesia. St. Dabeoc's Heath. ${ }^{3}$

Dact'ylis, L. Grämin' e.z, Grasses. Tri. Dig. glom'era'ta, L. 112. p. 6, 7. Rough Cock's-foot-grass. Clustered C. strict'a, Soland. = Spart'ina strict'a, Rotb.

Dam'aso'nium, Juss. Alisma'ees, Alismads. Hex. Polyg. stella'tum, ${ }^{9}$ Pers. 13. p. 5-7. Thrumwort. Star-fruit.

Daph'ne, L. THY' 'EL E' 'CE E, Daphnads. Oct. Mon. Laur'eola, L. 51. 8. 1-4. Spurge Laurel.

Meze'reum, L. 9. s. 2-4. Mezereon. Spurge Olive.

Datu'ra, L. So'laxi'ces, Night-shades. Pent. Mon. Stramo'nium, L. a. 7-10. Thorn-apple.

Dauc'us, L. UMBELLIF' ERE, Umbellifers. Pent. Dig. Caro'ta, L. 106. b. 6-8. Wild Carrot. Bird's-nest. v. gumm'ifer $=\mathrm{D}$. gumm'ifer, Lam. gumm'ifer, ${ }^{10}$ Lam. 17. b. 7, 8. Sea-side Carrot. marit'imus, With. $=$ D. gumm'ifer, Lam.

Delphi'nium, L. Rañonc'Ula'ces, Crowfoots. Poijan. Pent. Aja'cis, Reichb. a. 6, 7. Branching Larksputr. Consol'ida, L. a. 6, 7. Field Larkspur. Wild L.

Denta'ria, L.

bulbif'era, $\mathrm{L} .=$ Cardami'ne bulbif'era, R.Br.

\begin{tabular}{|c|c|c|}
\hline L.C. & $\begin{array}{c}\text { E.B. } \\
\text { iv. } 1 \cup 90\end{array}$ & $\begin{array}{l}\text { B. } \\
1196\end{array}$ \\
\hline 983 & & 709 \\
\hline $\begin{array}{l}982 \\
982 \mathrm{~b}\end{array}$ & vii. 1118 & 708 \\
\hline 1689 & xi.1776 & $12 \cdot 24$ \\
\hline 90 & $x i .1777$ & 1225 \\
\hline 1520 & $x .1577$ & \\
\hline 1520 & x.1577 & 1078 \\
\hline 1521 & $x .1578$ & 1077 \\
\hline 1369 & ix. 1490 & 1011 \\
\hline $\begin{array}{l}1786 \\
1786 \mathrm{~b}\end{array}$ & $\begin{array}{l}\text { xij. } 1866 \\
\text { xii.1867 }\end{array}$ & \\
\hline 1785 & xii.1864 & 1301 \\
\hline $1785 b$ & xii.1865 & \\
\hline 1787 & xii.1868 & $1 \div 02$ \\
\hline 326 & (iii.329) & $2: 30$ \\
\hline 927 & (vi.885) & $(632)$ \\
\hline 1696 & xi.1778 & 1223 \\
\hline 1474 & (ix.1442) & 968 \\
\hline 1240 & viii. 1247 & 878 \\
\hline 239 & viii. 1246 & 877 \\
\hline 1019 & vi.935 & 710 \\
\hline 672 & iv. 615 & 449 \\
\hline 673 & $\begin{array}{l}1 v .616 \\
\text { iv. } 616\end{array}$ & \\
\hline 14 & i. $47 \mathrm{~A}$ & 29 \\
\hline & i. $47 \mathrm{~B}$ & \\
\hline
\end{tabular}

1 Cynogolossnm sylraticnm. 2 Cyathea incisa,-Cystopteris regia. ${ }^{3}$ C. Dickienna, 4 Cyathea fragilis. -Cystea fragilis. 5 Cystea dentata.-Cystopteris dentata. 6 Saruthamnns scoparius.-Spartinm scoparium. -Genista scoparia. 7 Erica Dabeoci.-Menziesia polifolia. 8 Connemara Heath. 9 Actinocarpus Damasonium.-Alisma Damasoninm. 10 Daucus Carota, v. gummifer.-D. maritimns. 
Deschamps'ia, Bealiv. Grāmin'ex, Grasses. Tri. Dig. alpi'na, ${ }^{1}$ K. \& S. 13, p. 6, 7. Smooth Alpine Hair-grass. caspito'sa, ${ }^{2}$ Beauv. 111. p. 6, 7. Tufted or Turfy H. Hassocks. v. brev'ifol'ia, Purn.

v. pseud'o-alpi'na, Syme.

v. long'iarista'ta (Parn.)

flexuo'sa, Trin. 105. p. 6-8. Heath Hair-grass. Waved H.

v. monta'na (Huds.)

v. roirlichens'is, J. C. Melvill.

seta'cea, ${ }^{4}$ Hanbury. 11. p. 7, 8. Bog Hair-grass.

Deyeux'ia, Clarion. Grāmin'ex, Grasses. Tri. Dig.

neglect'a, ${ }^{5}$ Kunth. 2.p.6,7. Narrow Small-reed. Smallest Close.reed.

v. Hook'eri, ${ }^{6}$ Syme.

strigo'sa, Kunth. 1.

Dianth'us, L. Can'rophrlu'es, Cloveworts. Dec. Dig.

Arme'ria, L. 45. a. or b. 7, 8. Deptford Pink.

cæs'ius, Sm. 1. p. 6, 7. Cheddar Pink. Monntain P.

Car'yophy'l'us, L. p. 7. 8. Clove P. Wild Carnation. C. Gilly-flower. deltoi'des, L. 51. p. 6, 9. Maiden Pink.

v. glauc'us, L.

pluma'rius, L. p. 6.8. Common Pink.

pro'lifer, L. 8. a. 6-10. Proliferous P. Childing P. Limewort.

Digita'lis, Tourn. Scro'pulularin'ex, Figworts. Did. Ang. purpur'ea, L. 107. a. or b. 7.9. Purple F'ox-glove or Folk's-glove.

hŭmifu'sa, Pers. = Pa'nicum gla'brum, Gand. sanguina'lis, Beaur. = Pa'nicum sanguina'le, $\mathrm{L}$.

Digraph'is, Trin.

aruud'ina'cea, Trin. = Phal'aris arund'ina'cea, $\mathrm{L}$.

Dioscor'e.e, R.Br. Tams.

Dio'tis, Desf. Compos'ite, Composites. Syn. Eq. candidiss'ima, Desf. Sce marit'ima, Cass.

[Cotton-weed. marit'ima, ${ }^{7}$ Cass. [candidiss'ima, Desf.] 9. p. 8, 9. Sea-side

Diplotax'is, DC. CRuCif'ene, Crucifers. 'Tetrad. Siliq. mura'lis, ${ }^{8}$ DC. 46. a. b. or p. 8,9. Sand Rocket. Wall Mustard. v. Babingto'nii, Syme.

ten'uifol'ia, ${ }^{8}$ DC. 41. p. 6-9. Fine-leaved Wall Mustard or R. ${ }^{10}$

Dipsa'ce.e, Juss. Teazleworts.

Dips'acus, L. Dip's'Ace. Teazleworts. Tetran. Mon.

Fullo'num, Mill. b. 8, 9. Cultivated Tecisle. Fuller's T. pilo'sus, L. 51. b. 7-9. Small T. Shepherd's Stuff. sylvest'ris, Huds. 72. b. 7-9. Wild Teasle.

Do'roni'cum, L. Compos'ite, Composites. Syn. Sup.

Pard'alianch'ěs, L. p. 5-7. Great Leopard's-bane. plantägin'eum, L. p. 6, 7. Plantain-leaved $L$.

Drab'a, L. CrdCif'ere, Cmucifers. Tetrad. Siliq.

aizoi'des, L. 1. p. 3-5. Yellow Alpine Whitlow-grass. Sea-green W. brach'yearp'a. Jord. = Eroph'ila præ'cox, IC.

hirt'a, $\mathrm{Sm} .=\mathrm{D}$. rupest'ris, R. Br.

inca'na, L. 26. b. 6, 7. Twisted-podded W. Woolly W.11 v. confu'sa, Ehrh.

infla'ta, H. C. Wats. = Eroph'ila infla'ta, Hook. f.

mura'lis, L. 9. a. or b. 4,5 . Speedwell or Broad-leaved $W .{ }^{12}$ rupest'ris, ${ }^{13}$ R. Br. 5. p. 7, 8. Rock Whitlow-grass. Hairy $W$. vern'a, L. $=$ Eroph'ila vulga'ris, DC.

Dros'era, L. 1)Ros'ERA'CE

an'glica, Huds. 54. p. 7.8. Larger long-leaved Sundew. Great ${ }^{14}$

v. ob'ora'ta (M. \& K.).

\section{4.}

L.C. E.B.

1674 (xi.173I)

$1673(x i .1730)$

$1673 \mathrm{~b}$

$1673 \mathrm{c}$

$1673 \mathrm{~d}$

$1676(x i .1732)$

$1676 \mathrm{~b}$

$1676 \mathrm{c}$

1675

1666 (xi.1725)

$1666 \mathrm{~b} \times \mathrm{xi} .(1726)$

1665

184

ii. 191

186

188

185

$185 \mathrm{~b}$

187

189

ii. 193

ii 194

ii. 192

ii. 195

ii.196

1047

vi.952

126

746

xi.1691

B.

(1185)

(1186)

(1184)

128

130

129

xi. 1697

I 162

1388

ix.1508

760

ง.7.25

531

135

(i.94)

134

(i.93)

720

iv. 67

iv. 675

iv. 676

490

720

iv. 674

$4: 9$

$7 \% 9$

r.761

550

551

103

i. 138

88

j.134-2

89

j. 136

90

$101 \mathrm{~b}$

i. 134.3

i. 135

91

i.13\%

i.134.1

9.

575

ii. 183

$39 \mathrm{I}$

Aire a stricta.-Arundo stricta. 6 A. lapponica.-Calamagrngtis lapponica.-C. stricta, v. Houkeri. 7 Santolina maritima. 8 Brassica muralis. - Sinapis muralis, - Sisymbiuin mnrale. 9 S. tenuifolium.-Brassica tenuifolia.-Sinapis tenuifolia. 10 Sand Mustard. 11 Hoary Draba. 12 Wall Draba. 13 Draba hirta. 14 English Sundew. 
Dros'era, L.-continued.

intermed'ia, ${ }^{1}$ Hayne. 49. p. 7, 8. Lesser Long-leaved Sundew. ${ }^{2}$ v. subcaulesc'ens, Melvill.

longifol'ia, L. = D. intermed'ia, Hayne.

rotund'ifol'ia, L. 107. p. 7, 8. Round-leaved S. Red-rot.

Dros'era'CEs, DC. Sundews.

Dry'as, L. Rosa'ces, Roseworts. Icos. Pol.

depress'a, Bab. a var. of $\mathrm{D}$. octopet'ala, $\mathrm{L}$.

octopet'ala, L. 16. s. 6, 7. Mountain Avens. White Dryas. v. pilo'sa, Bab.

Ech'inoch'loa, Beauv.

Crus-gall'i, Beauv. = Pa'nicum Crūs-gall'i, $\mathbf{L}$.

Ech'inoph'ora, Tourn. Umbelli'́tere, Umbellifers. Pent. Dig. spino'sa, L. p. 7. Sea Prickly-Samphire or Parsnip.

Echi'nosperm'um, Sw. Boraorn'ex, Borageworts. Pent. Mon. Lapp'ula, Lehm. a. 6. Stick-seed.

Ech'ium, Tourn. Boñ̄ar'e.e, Borageworts. Pent. Mnn. plantâgin'eum, ${ }^{3}$ L. 1. a. or b. 6-8. Purple Viper's-bugloss. viola'ceum, Koch $=\mathbf{E}$. plantãgin'eum, $\mathrm{L}$. vulga're, L. 90. b. or a. Viper's-bugloss. Viper-grass.

Elati'nes, Camb. Water-peppers.

Flati'ne, L. Elati'xes, Water-peppers, Oct. Tet.

hexand'ra, DC. 18. a. 7-9. Small Hexandrous Water-wort. Hydrop'iper, L. 3. a. 7, 8. Small Octandrons W. Water-pepper. tripet'ala, Sm. = E. hexand'ra, IC.

EL $E^{\prime}$ AGNA'CE $\boldsymbol{E}$, Juss. Oleasters.

El'eoch'aris, Br. CYr'ÉRA'ce.e, Sedges. Tri. Mon. ac'icula'ris, ${ }^{5} \mathrm{Sm}$. 64. p. 7, 8. Slender Club-mish. Necdle C. crespito'sa, Reich. = Scirp'us crespito'sus, L.

multicau'lis, ${ }^{6} \mathrm{Sm}$. 77. p. 6. Many-stemmed Club-rush.

palust'ris, ${ }^{7}$ R. Br. 107. p. 6, 7. Marsh C. Spike-rush.

parv'ula, Hook. = Scirp'us parv'ulus. R. \& S.

pauciflo'ra, Link = Scirp'us pauciflo'rus, Lightf.

u'niglu'mis, ${ }^{8}$ Link. 29. p. 6, 7. Link's Club-rush. v. Watso'ni, ${ }^{8}$ (Bab.)

Watso'ni, Bab. = E. uniglu'mis, v. Watso'ni.

El'eogi'tōn, Link.

flu'itans, Link = Scirp'us flu'itans, L.

Elis'ma, Buchenau. Alisma'cez, Alismads. Hex. Pol. nat'ans, ${ }^{10}$ Buchenau. 13. p. 7, 8. Floating Water Plantain.

Elo'dea, Rich. H' Hrocnarid'ex, Hydrocharids. Diœc. Enn. canadens' is, ${ }^{11}$ Mich. p. 5-9. Water-Thyme. New Water-wced. ${ }^{12}$

El'ymus, L. Grãmn'ex, Grasses. Tri. Dig.

L.C. E. B. ar'ēna'rius, L. 30. p. 7. Sand Lyme-grass. Upright Sca L. europæ'us, $\mathrm{L}_{\mathrm{.}}=\mathrm{Hol}^{\prime} \mathrm{d}^{\prime} \mathrm{um}$ sylva'ticum, Huds. gen'icula'tus, Curt. p. 7. Drooping $L$. Pendulous Sea $L$.

Ely'na, Schrad. cārici'na, Mert. \& Koch. = Koobre'sia cārici'na, Willd.

Eis'petra'cee. Cranberries.

Em'petrum, L. Eu'petra'ce.e, Cranberrics. Diøe. Trian. ni'grum, L. 71. s. 4-6. Black Crowberry or Crakeberry.

Endym'ion, Dum. nu'tans, Dum. = Scill'a nu'tans, Sm.

Fp'ilob'ium, L. ON'AGra'ries, Onagrads. Oct. Mon. alpi'num, L. $21 .^{13}$ p. 7. Lesser Alpine Willow.herb. alsi'nefol'ium, Vill. 23. p. 7. Chickwed-leaved $W$.
576 ii. 184

$576 \mathrm{~b}$

574

574

477

$477 \mathrm{~b}$

615

xi. 1692

iv. 628

1007

1006

257

257

258

1241

1522

1525

1523

.1588)

(x.1586)

1524

$1524 b$

(x. 1587)

i. 262

ii. 263

viii. 1245

(x.1585)

(1085)

(1088)

(1087)

176

B.

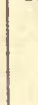

1472

(ix.1441)

\begin{tabular}{l|l|l}
1323 & $\mathrm{ix} .1446$ & 973
\end{tabular}

\begin{tabular}{l|l|l}
1760 & xi.1819 & 1200
\end{tabular}

viii.1251

1315 viii.1251

897

603 iv.507

348

601 iv.505
1 Drosera longifolia.

4 Elatine tripetala.

9 Eleocharis Watsoni.

${ }^{2}$ Spathulate-leaved Sundew._Intermediate Sundew. Scirpus acicularis. 10 Alisma nataus.

6 S. mnlticaulis.

11 Anacharis alsinastram.
3 Echinm violaceum. $8 \mathrm{~S}$. uniglumis. 
Ep'ilob'ium, L.-continued.

anagall'idiful'ium, Lam. 21.' p. 7. Greater Alpine $W$.$$
\text { I. }
$$

lanceola'tum, Seb. \& Maur. p. 7-9. Spear-leaved Willow-herb. monta'num, L. 112. p. 6, 7. Broad Smooth-leaved W. obscu'rum, ${ }^{3}$ Schreb. 82. p. 7, 8. Short-podded Square-stalked W. palust're, L. 104. p. 7, 8. Narrow-leaved Marsh W.

v. lig'ula'tum, Baker.

parviflo'rum, Schreb. 101. p. 7, 8. Small-flowered Hairy $W^{*} .^{5}$

v. ri'vula'ro (Wahl.)

r. intermed'jum (Mérat.)

ros'eum, Schreb. 38. p.7,8. Small-flowered Pale Smooth-leaved W. rosmari'ni fol'ium, Hacnke. p. 8. Rosemdry-leaved French $W$. tetrago'num, L. 33. p. 7, 8. Long-podded Square-stalked W. virga'tum, Gren. \& Godr.= E. obscu'rum, Schreb.

Ep'ime'dium, L. Berberid'ex, Berberids. 'Tetran. Mon. alpi'num, L. p. 5. Alpine Barren-wort.

Ep'ipact'is, Crantz. ORCнin's, Orchids. Gyn. Mon. a'tro-rub'ens, ${ }^{6}$ Schultz. 8. p. 7. Oval-leaved Helleborine. ensifol'ia, Sw. = Ceplı́alanthe'ra ensifol'ia, Kich. grandifo'ra, Sw. = Ceph'alanthe'ra pall'ens, Kich. Hellebori'ne, Crantz.

la'tifol'ia, Auct. $85 .^{8}$ p. 7, 8. Broad-leaved Helleborine. med'ia, Fr. $85 .^{9}$ p. 8. Narrow-leaved Helleborine. ora'lis, Bab. = E. a'tro-rub'ens, Schultz. palust'ris, ${ }^{10}$ Crantz. 62. p. 7. Marsh Helleborine. purpura'ta, "Sm. 35. p. 8. Purple-leaved Hellebmine. rubi'gino'sa, lioch $=\mathrm{E}$. a'tro-rub'ens, Schultz. ru'bra, Sw. = Ceph'alanthe'ra ru'bra, Rich. viola'cea, Bur. = E. purpura'ta, Sm.

Epipo'gum, Gmel. Orcuv'Ex, Orehids. Gyn. Mon. aphyll'um, ${ }^{12} \mathrm{Sw}$. 2. p. 8. Leafless Epipogium. Gmeli'ni, Rich. = E. aphyll'um, Sw.

EQ'UISETA'CE.E, DC. Horsetails.

Eq'uise'tum, L. FQ'useta'ce.e, Horsetails. Crypt. Fil. arvens'e, L. 108. p. 4. Corn or Field Horsetail. I)rummond'ii, Huok. = E. pratens'e, Ehrb. fluv'ia'tile, $\mathrm{Sm}$. = E. max'imum, Lam. hyema'le, L. 41. p. 7, 8. Gtr. Rough H. Dutch Rush or Shavegrass. limo'sum, Sm. 103. p. 6, T. Water $H$. Smooth Naked $H$. v. fluv'ia'tile (L.)

littora'le, Kühl. 1.

Macka'ii, Newm. $=$ E. tra'chyodōn, A. Br.

max'imum, ${ }^{13}$ Lam. 77. p. 4. Great Water Horsctail.

v. sero'tinum, A. 131 .

v. Braun'ii, Mrilde.

Moore'i, Newm. p. 7, 8. Moore's Horsetail.

palust're, L. 104. p. 6, 7. Marsh Horsetail.

r. polystach'yum, Auct.

praten's', 14 Ehrh. 19. p. 4. Blunt-topped Horsetail. ramo'sum, Bentl. $=\mathrm{E}$. tra'chyod'ōn, A. Br. sylva'ticum, L. 90. p. 4, 5. Branched Wood Horsetail. 'Telmatei'a, Ehrh. = E. max'imum, Lam.

\begin{tabular}{|c|c|c|}
\hline $\begin{array}{l}\text { L.C. } \\
602 \\
590\end{array}$ & $\begin{array}{l}\text { E.B. } \\
\text { iv.506 }\end{array}$ & 340 \\
\hline & iv. 495 & \\
\hline $590 \mathrm{~b}$ & iv.496 & \\
\hline $\begin{array}{l}592 \\
599\end{array}$ & iv.497 & 341 \\
\hline 595 & iv. 500 & \\
\hline 594 & iv.499 & $3+3$ \\
\hline 598 & iv. 503 & \\
\hline 600 & iv.504 & 346 \\
\hline $600 \mathrm{~b}$ & & \\
\hline 593 & iv.498 & $3 \pm 2$ \\
\hline $\begin{array}{l}593 \mathrm{~b} \\
593 \mathrm{c}\end{array}$ & & \\
\hline 536 & iv. 501 & 344 \\
\hline 591 & iv. 494 & \\
\hline 597 & iv. 502 & 345 \\
\hline 49 & i 52 & \\
\hline 1343 & ix.1481 & \\
\hline & ix.1479-81 & \\
\hline 1340 & ix.1480 & 979 \\
\hline 1341 & ix.1479 & \\
\hline 1344 & ix.1482 & 980 \\
\hline 1336 & ix.1486 & 987 \\
\hline 1811 & xii. I 888 & \\
\hline 1812 & xii.1889 & 1260 \\
\hline 1818 & xii.1 894 & 1265 \\
\hline $\begin{array}{l}1817 \\
1817 \mathrm{~b} \\
1816\end{array}$ & xii. 1893 & $1 \div 63$ \\
\hline 1811 & xii. 1888 & $(1259)$ \\
\hline $\begin{array}{l}1811 \mathrm{~b} \\
1811 \mathrm{c}\end{array}$ & & \\
\hline 1819 & xii.1895 & \\
\hline $\begin{array}{l}1815 \\
1815 \mathrm{~b}\end{array}$ & xii.1892 & 1264 \\
\hline 1813 & xii. 1890 & 1262 \\
\hline & rii 1891 & $\begin{array}{l}1266 \\
1261\end{array}$ \\
\hline & X11.1891 & 1259 \\
\hline
\end{tabular}

1 Including E. alpinum. Willow-herb. 8 Including Epipaclis media. 12 Epipogum Gmelini.
2 Codlins \& Cream.

3 Epilobinm virgatum. (llow-herb. Eipsctis rubiginosa - E. ovalis. $\theta$ Including E. latifilia. 10 Serapias palustris.
4 Round-stalked Marsh 7 Serupias latifolia. 11 Epipactis violacea. 
Eq⿱ $\mathfrak{q}^{\prime}$ ise'tum, $\mathrm{L}$ - continued.

tra'chyod'ön, 'A. Br.p. 7,8. Long-stemmed Horsctail. Mackay's $H$. umbro'sum, Willd. $=$ E. pratens'e, Ehrh.

variega'tum, Schleich. 24. p. 7, 8. Variegated Rough $H$.

v. ar'ēna'rium, Newm.

v. ma'jus, Syme.

v. Wilso'ni, ${ }^{2}$ Newm.

Wilso'ni = E. var'iega'tum, v. Wilso'ni.

Eranth'is, Salisb. Raxusc'ula'ce.e, Crowfoots. Polyan. Pol. hyema lis, Salisb. p. 1-3. Winter Aconite.

Eri'ca, L. ER'ícA'CEx, Heathworts. Oct. Mon.

carn'ea, L. = E. mediterra'nea, L.

cilia'ris, L. 3.s. 8, 9. Fringed-leaved Heath.

ciner'ea, L. 108. s. 7-9. Fine-leaved H. Grey H. Scotch Heather.

Dabeoc'i, Sm. = Dabœ'cia pol'ifol'ia, D. Don,

hibern'ica, Syme. $=$ E. mediterra'nea, $L$.

Mackaia'na.' Hook. I. s. 8, 9. Mackay's Heath.

Mackay'i, Hook. = E. Mackaia'na, Hook.

mediterra'nea, ${ }^{4}$ L. I. Irish Heath. Mediterrancan $H_{.}{ }^{3}$

Tet'ralix, L. 109. s. 7-9. Cross-leaved Heath.

Tetral'ici-cil'ia'ris, Syme. = E. Watso'ni, Benth.

rag'ans, L. 1. s. i, 8. Cornish Heath.

vulga'ris, L. = Callu'na Eri'ca, DC.

Watso'ni, ${ }^{6}$ Benth. 2. Hybrid.

ER'iCA'OE.s. Heathworts.

Erig'erōn, L. Compos'rt.e, Composites. Syn. Sup.

a'cre, L. 65. a. or b. 7, 8. Blue Fleabane.

alpi'num, ${ }^{7}$ L. 3. p. 7, 8. Alpine Fleabane.

canadens'e, L. a. 8, 9. Canadian Fleabane. n'niflo'rum, Sm. = E. alpi'num, L.

Eri'nus, L. Scro'puularin'e. Figuorts. Did. Ang. alpi nus, L. p. 7. Alpine Erinus.

Er'iocat'LONex, Rich. Pipeworts.

Er'iocau'lon, L. Er'iocaulon'e e, Pipcworts, Monœc. Hex. septang'ula're, With. 2. p. 8. Jointed Pipewort.

Erioph'orum, L. Crp'era'ces, Sedges. Tri. Mon.

alpi'num, L. 2. p. 5. Alpine Cotton-grass.

angust 'ifol'ium, ${ }^{8}$ Roth. 109.p. 4, 5. Narrow-leaved Cotton-grass.

v. ela'tius, Koch.

v. min'us, Koch.

eap'ita'tum, Host. p. 6. Round-headed Cotton-grass.

grac'ile, Koch. 3. p. 6, 7. Slender Cotton-grciss.

la'tifol'ium, ${ }^{9}$ Hoppe. 51. p. 5, 6. Downy-stalked Broad-leaved $C$. polystach'ium, L. $=E$. angust'ifol' ium, Roth.

polystach'ium, Sm. = E. la'tifol'ium, Hoppe.

pubesc'ens, Sm. = E. la'tifol'ium, Hoppe.

vagina'tum, L. 89. p. 4, 5. Hare's-tail $C$. Sheathing $C$.

Ero'dium, L. Gerax'ia'ce.e, Crane's-bills. Monad. Pent. cic'uta'rium, L' Hérit. 99. a. or b. 6-9. Hemlock Stork's-bill. ${ }^{10}$

v. vulga'tum, Syme.

v. chrerophyll'um (Cav.)

marit'imum, L' Hérit. 33. a. or b. 5-9. Sea Stork's-bill. moscha'tum, L' Hérit. 11. a. 6, 7. Musk Stork's-bill.

Eroph'ila, DC. Crucir'ERs, Cmicifers. 'Tetrad. Siliq. brach'ycarp'a, Jord. = E. præ'cox, DC.

infla'ta, 11 Inok. f. 1. a. or b. 7. Whitlow-grass.

præ'cox, ${ }^{12}$ DC. 29. a. or b.

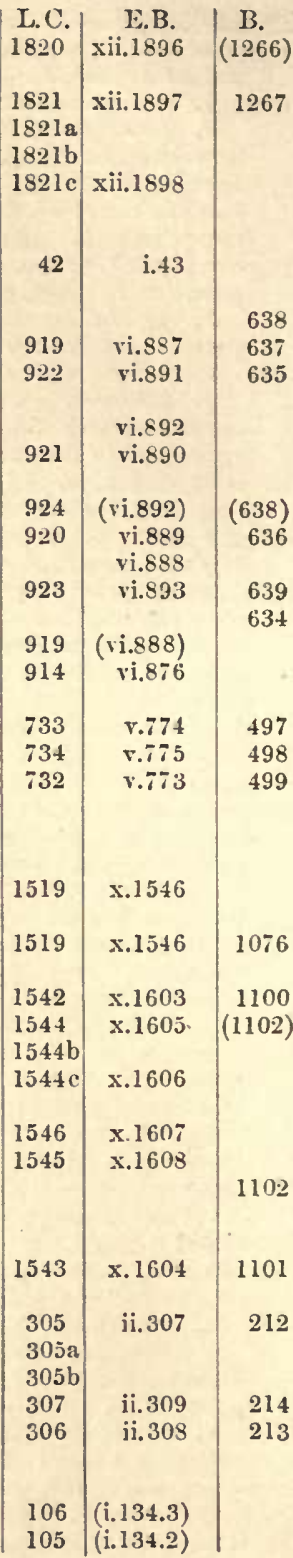

I Equisetum ramosum.-E. Mackaii, 2 E. Wilsoni, 3 Erica Mackayi. 4 E. hibernica,-F. carnea. 5 Flesh-coloured Heath. 6 Erica Tetralici-riliaris. 7 Erigeron uniflorum. 8 Ei iophorum polystacbium, L. 9 E. polystachinm 8 m.-E. pubescens. 10 Heron's Bill. 11 Draba infata. i2 D. bracbyearpa.-Erophila brachycurpa. 
Eroph'ila, DC.-continued.

veru'a, L. = E, vulga'ris, DC.

vulga'ris, ${ }^{1}$ ])C. 99. a. or b. 3-5. Whitlow-grass or Cress. ${ }^{2}$

Erv'um, L.

hirsu'tum, L. = Vic'ia hirsu'ta, Koch.

tetrasperm'um, L. = Vic'ia tetrasperm'a, Moench.

Eryng'ium, L. Úmbellif'er.e, Umbellifers. I'ent. Dig. cumpest're, L. 7. p. 7, 8. Field Eryngo. marit'imum, L. 50. p. 7, 8. Sea Eryingo. Sea Holly.

Erys'imum, L. Crucif'ere, Crucifers. Tetrad. Siliq.

Allia'ria, $\mathrm{L}_{4}=$ Sisymb'rium Allia'ria, Scop.

Barbare'a, L. = Barbare'a vulga'ris, K. Br.

cheiranth'oï'des, L. 37. a. 6-8. Treacle Hedge Mustard. ${ }^{3}$

offic'īna'le, L. = Sisymb'rium offic'ina'le, Scop.

omenta'le, ${ }^{4}$ R. Br. [per'folia'tum, Cr.] a. 6. Hare's-ear Cabbag?: per'folia'tum, Crant: see orienta'le, R. Br.

præ'cox, Sm. = Barbar'ea præ'cox, R. Br.

Er'ythræ'a, Renealm. Gevtiana'ce. Gentianworts. Pent. Mon. [cap'ita'ta, Willd.] Tounsend's Centaury.

v. spharoceph'aja, Towns. 2.

Centaur'ium, ${ }^{6}$ Pers. 98. a. or b. 6-9. Common Centaury. r. capita'ta (lioch)

la'tifol'ia, Sm. 1. a. or b. 7. Broad-leaved Tufted Centaury. liua'rafol'ia, Pers. = E. littor'alis, Fr.

litura'lis, ${ }^{7}$ Fr. 38. a. or b. 6,7. Narrow-leaved C. Duarf Tufted $C$. pulchell'a, ${ }^{8}$ Fr. 40. a. or b. 8, 9. Slender C. Dwarf Branched C. v. ten'uiflo'ra (Link)

Eufra'gia, Griseb. visco'sa, Benth. = Barts'ia viser'sa, L.

Euo'nymus, L. Celastrix'E.e, Spindle-trees. Pent. Mon. europæ'us, L. 71. t. 5, 6. Spindle-tree.

Eupator'ium, 'lourn. Cospos'Ite, Composites. Syn. Eq. cannab'inum, L. 97. p. 7-9. Hemp-Agrimony.

Euphorb'ia, L. Eupuorn'ia'cex, Spurgeworts. Monœe. Mon. amyg'daloï'des, L. 51. p. 3-5. Wood Spurge.

Charac'ias, L. s. 3, 4. Red Shmibby Spurge.

corall'ö̈'des, L. b. 5, 6. Coral Spurge.

Cypariss'ias, L. p. 6, 7. Cypress Spurge.

dulc'is, L. p. 6.

E'sula, L. p. 7. Leafy-branched Spurge.

v. Pseud'o-cypariss'ias, Jord.

exig'ua, L. 81. a. 7-10. Dwarf Spurge.

He'lioscop'ia, I. 106. a. 6-10. Sun-Spurge. Wart-wort.

hibern'a, L. 1. p. 5, 6. Irish Spurge.

La'thyris, L. b. 6, 7. Caper Spurge.

palust'ris, Bab. = E. pilo'sa, L.

Paral'ias, L. 29. p. 7-10. Sea Spurge.

Pep'lis, L. 9. a. 7-9. Purple Sprorge.

Pep'lus, T. 98. a. 7-11. Petty Spurge.

pilu'sa, ${ }^{9}$ L. 2. 2. 5, 6. Downy Spurge. Hairy $S$.

platyphyll'os, L. 28. a. 7-10. Broad-leaved Warted $S$.

portland'ica, ${ }^{10}$ L. 19. p. 5-8. Portland Spurge.

seg'eta'lis, Bentb. = E. portland'ica, L.

strict'a, L. 2. a. or b. 8, 9. Bushy Warted Spurge.

Eurnorbia'ce.E, Juss. Spurgeworts.

\begin{tabular}{c|c|c} 
L.C. & E.B. & B. \\
104 & $($ i. 134.1) & $(92)$
\end{tabular}

(ir.

612

iv. 570

399

611

iv. 569

398

119

i. 102

Euphra'sia, Tonrn. Scko'pululinix'Ex, Figworts. Did. Ang. Ud'onti'tes; L. = Barts'ia Ud'onti'tes, IIuds. 
Euphra'sia, Tourn.-continued.

offic'inna'lis, L. 111. a. 5-9. Eyebright.

v. grac'ilis (Fr.) 3. marit'ima, Hook. 2.

Ex'acum, L.

filiform'e, Sm. = Mi'crocal'a fi'liform' is, Link.

Fa'gopy'rum, Tourn. Poz'rgōxs'ce.s, Buckwheats. Oct. Trig. esculent'um, ${ }^{1}$ Moench. a. 7-9. Buckwheat. Branks.

Fa'gus, Tourn. Cu'pulif'er.s. Monœe. Pol.

Ca: $\tan ^{\prime}$ ea, $\mathrm{L} .=$ Castan'ea sati'va, Mill.

sylva'tica, L. 64. t. 4, 5. Common Beech.

Falca'tula, Brot.

orni'thopod'ioídes, Bab. = Trigonell'a purpurasc'ens, Lam.

Fe'dia, Moench.

Auric'ula, Gaud. $=$ Valer'ianeli'a Auric'ula, DC.

carina'ta, Stev. = Valer'ianell'a carinna'ta, Lois.

denta'ta, Vahl. = Valer'ianell'a denta'ta, Poll.

olito'ria, Vahl. = Valer'ianell'a ölito'ria, Moench.

Festu'ca, L. Grïmix'E.e, Grasses. Tri. Dig.

ambig'ua, Le Gall. 6. a. 6. Ambiguous Fescue-grass.

ar'êna'ria, Osb. =F. ru'bra, v. ar'êna'ria.

arund'ina'cea, ${ }^{2}$ Schreb. 84.

bromoï'des, Sm. = F. sciu'roïdes, Roth.

Calama'ria, $\mathrm{Sm} .=\mathrm{F}$. sylva'tica, Vill.

cæ'sia, $\mathrm{Sm} .=\mathrm{F}$. ovi'na v. glaue'a.

decid'ua, Sm. $=$ F. sylva'tica, v. decid'ua.

dumeto'rum, $\mathbf{L}$.

du'riusc'ula, $\mathrm{L}:=\mathrm{F}$. fall'ax, Th.

ela'tior, ${ }^{3}$ L. 90. p. 6, 7. Tall Fescuc-grass.

v. pseud'o-lólia'cea, Hack.

v. lŏlia'cea, ${ }^{4}$ Huds.

v. pratens' is, ${ }^{,}$Auct.

v. arund'ina'cea $=$ F. arund'ina'cea, Schreb.

fall'ax, ${ }^{6}$ 'Th. 99. p. 6 . Hard Fescue-grass.

gir'ante'a, Vill. = Brom'us gig'ante'us, L.

lölia'cea. ${ }^{7}$ 38, a. 7, 8. Spiked Fescue-grass.

myu'rus, ${ }^{8}$ L. 46. a. 6. Wall Fescue-grass. Mouse-tail F.

ora'ria, Dum. = F. ru'bra, v. ar'èna'ria.

ovína, L. 110. p. 6, 7. Sheep's Fescue-grass.

v. eapilla'ta, ${ }^{9}$ Hack.

v. glauc'a, ${ }^{10} \mathrm{Sm}$. or Lam.

v. ten'uifol'ia, Sibth. =v. capilla'ta, Hack.

pinna'ta, Huds. = Brach'ypod'ium pinna'tum, Beauv.

pratens'is, Huds. = F. ela'tior, v. pratens'is.

v. lŏlia'cea $=\mathrm{F}$. ela'tior, $v$. lŏlia'cea.

procumb'ens, ${ }^{11}$ Kunth. 25. b.or p. 6,7. Procumbent Meadow-grass.

pseud'o-myu'ros, Soy.-Will. $=$ F. myu'rus, $\mathrm{L}$.

rig'ida, ${ }^{12}$ Kunth. 69, a. 6. Hard Meadow-grass.

ru'bra, L. 60. Creeping Fescue-grass. Red Fescue. v. ar'ena'ria, ${ }^{13}$ Osb.

scin'rni'des, ${ }^{14}$ Roth. 98. a. 7. Barren Fescue-grass.

sylva'tica, ${ }^{15}$ Vill. 28. p. 7. Slender Wood $F$. Reed $F$.

v. decid'ua. ${ }^{16}$

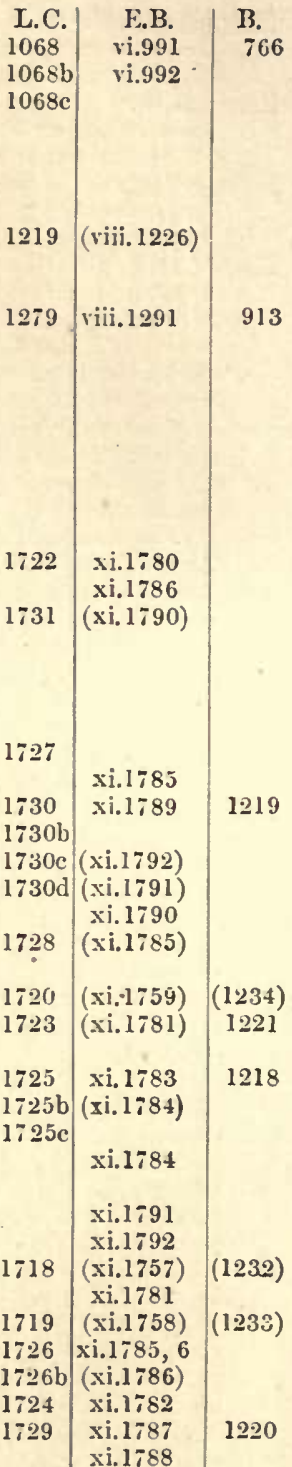

2 Polrgonum Fagoprrum. 2 Festuca elatior, r. arundinacea. 3 Schedonorus elatior. 4 Festuca prateusis, v. loliacea. 5 F. pratensis.-Schedonorus pratensis. 6 Festuca duriuscuia. 7 Catapodium Juliaceum.-Glycteria loliacea, - Pus loliacen. - Schedonorus loliaceus. - Sclerochloa loliacea. -Triticum loliacenm. 8 Vulpia myuros.-Festuca pseudo-myuros. $9 \mathrm{~F}$. vivipara,-F. ovina, v, tenuifolia. $10 \mathrm{~F}$. caesia. 11 Glyceria procumbens.-Pon procumbens. - Sclerochloa procumbens. 12 Glyceria rigida.-Pos rigida Sclerochloa rigida. 13 Festuca areania,-F. oraria. 14 F. bromoides, - Vulpia bromoides. 15 Festuca Calamaria.-Schedonorus sylvaticus. 16 Festuca decidua, 
Festu'ca, L.-continued.

triflo'ra, Sm. = Brom'us gig'ante'us, v. trîflo'rus.

u'niglu'mis,' Soland. 19. a. 6, 7. Single-glumed Fescue-grass. vivip'ara $=\mathrm{F}$. ovi'na, v, capilla'ta.

Fibich'ia, Koel. Gramix'E.e, Grasses. Tri. Dig. umbell'ata," Koel. 3. p. 7, 8. Creeping Dog's-tooth-grass.

Fica'ria, Dill. vern $\Omega$, Huds. = Ranunc'ulus Fica'ria, L.

Fila'go, 'Tourn. Compos'ır.e, Composites. Syn. Sup. ap'icula'ta, G. E. Sm. 18. a. 10. Red-tipped Cudweed. gall'ica, ${ }^{3}$ L. a. 6-9. Narrow-leaved Cudweed. germa'nica, ${ }^{4}$, 93. a. 7, 8. Common Cudweed. min'ima, ${ }^{3}$ Fr. 88, a. 6-9. Slender $C$. Field $C$. Least $C$. spathula'ta. Presl. 23. a. 10. Spathulate Cudwecd.

Fin'ices, L. Ferns.

Fœnic'ulum, Hotfm. Umbellif'erse, Umbellifers. Pent. Dig. capilla'ceum, Gilib. See oftic'înale, All. offic'inale, ${ }^{6}$ All. [capilla'ceum, Gilib.] 32, p. 7, 8. Fennel. vulga're, Gart. = F. uffic'ina'le, All.

L.C.
1721

Fraga'ria, L. Rosa'ces, Roseworts. Icos. Pol. a'tro-vir'ens, p. 5-9. A var. of $\mathrm{F}$. vesc'a, L. colli'na, Ehrh.

ela'tior, ${ }^{7}$ Ehrh. p. 4, 5. Hautbois Strawberry. moscha'ta, Duch. = F. ela'tior, Ehrl.

ster'ilis, L. = Pot'entill'a Fraga'rias'trum, Elrh. vesc'a, L. 111. p. 4, 5. Wood Strawberry.

Franke'nia, L. Fraxín'sin'ces, Frankeniads. Hex. Mon. lev'is, L. 12. p. i, 8. Smooth Sea-heath. pulv'erulent'a, L. a. 7. Powdery Sea-heath.

Fraxke'NiA'CEe, St. Hil. Frankeniads.

Frax'inus, Tuurn. Or'es'cex, Oliveworts. Dian. Mon. excels'iur, L. 109. t. 4, 5. Common Ash.

v. het'erophyll'a (IVilld.) [divers'ifol'ia, Ait.] Simple-leaved

Fritilla'ria, Tourn. II'LIA'CE., Lilyworts. Hex. Mon. [Ash. Mĕlea'gris, L. 20. p. 5. Fritillary. Chequered Daffodil. ${ }^{\triangleleft}$

Fumária, L. Fuma'ria'ces, Fumeworts. Diad. Hex.

Boræ'i, Jord. = E. pallidiflo'ra, v. Boræ'i.

cap'reola'ta, L.

cla'vicula'ta, L. = Coryd'alis cla'vicula'ta, DC.

confu'sa, Jord. 33. a. 6-9. Bastard's Rampant Fumitory. densiflo'ra, ${ }^{8}$ DC. 36. a. 5-9. Close-flowered Fumitory.

lu'tea, L. = Coryd'alis lu'tea, DC.

mīcranth'a, Lag. = F. densiflo'ra, DC.

mura'lis, Sond. 23. a. 8, 9. Rampant Fumitory.

offic'inna'lis, L. 105. a. 5-9. Common Fumitory. Earth-smoke. pallidiflo'ra, Jord. 28. a. 6-9. Pale-flowered Fumitory.

v. Boræ' $1,{ }^{10}$ Jord. 47. Boreau's Rampant $F$.

parvifl'ora, Lam. 21. a. 6-9. Lamarck's Small-flowered $F$.

sol'idla, Sm. = Coryd'alis bulbo'sa, DC.

ten'uisect'a, Syme.

Vaill'ant'ii, Loisel. 12. a. 6-9. Le Vaillant's Small-flowered F. FUMA'RIA'CEA, DC. Funcworts.

Ga'gea, Salisb. LI'LIA'Crx, Lilyworts. Hex. Mon.

fasc'icula'ris," Salisb. 41. p. 3-5. Yellow Star of Bethlehem.

lu'tea, Ker. $=$ G. fasc'icula'ris, Salisb.
1685

(xi.1690)

E.B.

xi.1759

736

739

735

738

737

1761

648

B.

1222

(1196)

v. 737

v.740

v. 736

v. 739

v.738

xii. 1838

(iv.601)

ir.601

482

iii. 439

481

iii. 438

182

ii. 190

ii. 190

18.

961 vi.902

vi. 903

1417

ix. 15 i9

i. .2

i. $71-4$

67

i. .73

(i. 75$)$

i. 75

i. 74

i. 6

i. 71

(i.72)

i.is

i.7.7.8

i. 77

i. 68

1419

(ix.1522)

ix.1522
(425)

425

310

126

667

1032

45

504

502

503

(

(1035)

1035

1 Vulpia uniglumis. 2 Cynodon Dactylon.-Panicum Dactylon. 3 Gnaphalium gallicum. 4 G. germanicum. 5 G. arvense.-G. minimum. 6 Anethum Fóniculum.-Fœniculum vulgare. - Mleum Foniculum. 7 Fragaria moschata. 8 Snake's head. 9 Fumaria micrantha. 10 F. Borai. $11 \mathrm{Gagea}$ ntea.-Ornithogalum luteum. 


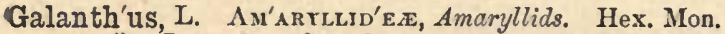
niva'lis, L. p. 1-3. Snowdrop.

Ğ̈leob'dolon, Adans. lu'teum, Huds. = La'mium Galeob'dolon, Crantz.

Găleops'is, L. LAв'IA'Tx, Labiates. Did. Gym. angust'ifol'ia, Ehrh. a. 9. Narrow-leaved Hemp-nettle. dıb'ia, ${ }^{1}$ Leers. 8. a. 7, 8. Downy Hemp-nettle. intermed'ia, Vill. a. 9. Intermediate Hemp-nettle. La'danum, L. 73, a. 7-10. Red Hemp-nettle. o'chroleuc'a, Lam. = G. dub'ia, Leers. spec'io'sa, ${ }^{2}$ Mill. 80. a. 7, 8. Large-flowered H. Bee-nettle. Tet'rahit, L. 112. a. 7-9. Common Hemp-nettle. v. bif'ida (Boenn.) versic'olor, Curt. = G. spec'io'sa, Mill. villo'sa, Huds. = G. dub'ia, Leers.

Galinso'ga, Ruiz \& Pav. Cospos'rt s, Composites. Syn. Sup. parvifto ra, Cav, a. 7, 8. Small-flowered Galinsoga.

Galium, L. RuB'iA'Ce. Stellates. Tetran. Mon. an'glicum, ${ }^{3}$ Huds. 10. a. 6, 7. Wall Bedstraw. Apari'ne, L. 112, a. 6, 7. Goosegrass. Cleavers. arista'tum, Sm. $=\mathrm{G}$. erect'um, v. arista'tum.

borea'le, L. 42. p. 6-8. Cross-leaved B. Northern Galium. ciner'eum, Sm. $=$ G. diffu'sum, Hook. commuta'tum, Jord. p. 8.

Crucia'ta, Scop. 95. p. 4-6. Crosswort. Maywort. Mugwort. diffu'sum, ${ }^{4}$ Hook. p. 8. Diffuse Bedstraw. Grey $B$. ela'tum, Thuill. = G. Mollu'go, v. ela'tum.

elonga'tum, Presl. = G. palust're, v. elonga'tum. erect'um, Huds. 24. p. 6, 7. Narrow-leav'd Great B. Upright B. v. arista'tum. ${ }^{\circ}$ Bearded Bedstraw.

Mollı'go, L. 75. p. 7, 8. Great Hedge B. Whip-tongue.

v. ela'tum ${ }^{6}$ (Thuill.)

v. insub'ricum (Gaud.)

v. Bake'ri, Syme.

monta'num, Vill. A var. of G. sylvest're, Poll.

palust're, L. 112, p. 7, 8. Marsh B. White Water Bedstraw. v. elonga'tum ${ }^{7}$ (Presl.)

v. Withering ${ }^{\prime}{ }^{8}{ }^{8}(\mathrm{Sm}$.)

parisiens'e, L. $=$ G. an'glicum, IIuds. pusill'um, sm. $=$ G. sylvest're, Poll. sacchara'tum, All. a. 6-8. Warty-fruited Bedstraw. saxa'tile, L. 111. p. 7, 8. Smooth Heath Bedstraw. sca'brum, With. = G. Mollu'go, v. ela'tum. spur'jum, L. a. 6, 7. Smooth-fruited Corn $B$. sylvest're, ${ }^{9}$ Poll. 22. p. 7, 8. Slender Bedstraw. v. nitid'ulum ('Thuill.)

tricor'ne, With. 42. a. 6-10. Rough-fruited Corn B. ${ }^{10}$ üli'gino'sum, L. 89. p. 7, 8. Rough Marsh Bedstraw. Vaillant'ii, DC. 1. a. 8-10. Hispid-fruited Corn B. ve'rum, L. 109. p. 6-9. Yellow B. Lady's B. Checse Rennet. v. o'chroleu'cum, Syme.

Withering'ii, Sm. = G. palust're, v. Withering'ii.

Gastrid'ium, Beauv. Grämin'en, Grasses. Tri. I'ig. austra'le, Beauv. See lendig'erum, Gaud. lendig'erum, ${ }^{11}$ Gaud. [austra'le, Beauv.] 24, a. 6-10. Awned Genist'a, L. Legu'Mino's $x$, Leguminous Plants. Diad. Dec. an'rlica. L. 86. s. 5, 6. Needle Furze. Petty Whin. Green-Weed. pilo'sa, L. 6. s. 5-9. Hairy Green-Weed.

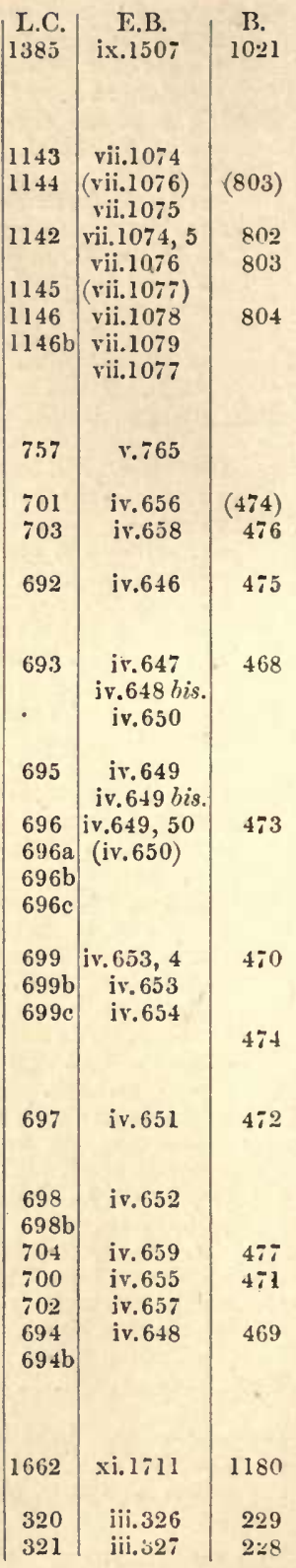

1 Galenpsis ochrolenca,-G. villosa. 2 G. versicolor. 3 Galium parisiense. 4 Galinm cinereum. $5 \mathrm{G}$. aristatum. $6 \mathrm{G}$. elatum,-G. scabrum. 7 G. elongatum. $8 \mathrm{G}$. Witheringii. 9 G. pusillum. 10 Three-flowered Goose-grass. 11 Agrostis australis. - Mitium lendigerum. 
Genist'a, L.-continued.

scopa'ria, Lam. = Cyt'isus scopa'rius, Link.

tincto'ria, L. 73. s. 7-9. Dyer's G. Woad. Woad-waxen. v. hŭmifu'sa, Dickson.

Gentia'na, Tourn. Gextis'xes, Gentianworts. Pent. Dig. acau'lis, L. p. 6, 7. Dwarf Gentian.

Amarell'a, L. 81. a. 7-9. Autumnal G. Small-flowered G.' v. prie'cox, Raf.

campest'ris, L. 85. a. 7-10. Field Gentian.

germa'nica, Willd. 9. German Gentian.

niva'lis, L. 2, a. 8, 9. Small Alpine Gentian.

Pneumunan'the, L. 30. p. 8, 9. Calathian Violet. Marsh G. vern'a, L. 4. p. 5, 6. Spring Gentian.

Gextia'nex, Juss. Gentianworts.

Geran'ta'ce.e, Juss. Cranesbills.

Geran'ium, L. Geran'ra'Ces, Cranesbills. Monad. Dec.

columbi'rum, L. 74. a. or b. 6, 7. Long-stalked Cranesbill.

rlissect'um, L. 104. a. or b. 5-8. Dove's-foot. Jagged-leaved $C$. lu'cidnm, L. 89. a. or b. 5-8. Shining Cranesbill. v. $R a^{\prime} i i^{2}$ Lindl.

moll'e, L. 112. a. or b. 5-9. Soft Cranesbill. Dove's-foot C. nodo'sum, L. p. 5-8. Knotty Cranesbill.

perenn'e, Huds. = G. pyrena'icum, L.

phe'um, L. p. 5, 6. Dusky Cranesbill.

pratens'e, L. 85. p. 6-9. Blue Meadow Cranesbill.

prostra'tum, Cav. = G. sanguin'eum, v. prostra'tum.

pusill'um, L. 75. a. 6-9. Sinall-flowered Cranesbill.

pyrena'icum, ${ }^{3}$ Burm. f. 55. p. 6-8. Pyrenean C. Monntain C.

Ra'ii, Lindl. $=$ G. ln'cidum, v. Ra'ii.

Robert'ia'num, L. 109, a. or b. 5-9. Herb Robert. Stinking $C$.

v. modest'um (Jord.)

v. purpur'eum, Auct. Angl.

rotund'ifol'ium, L. 21. a. or b. 6, 7. Round-leaved Cranesbill. sanguin'eum, L. 62. p. 7, 8. Crimson C. Blood Geranium.

v. prostra'tum ${ }^{5}$ (Cav.)

v. lancastriens'e (With.)

stria'tum, L. [versićolor, L.] p. 8. Pencilled Geranium.

sylra'ticum, L. 51. p. 6, 7. Wood Cranesbill.

versi'color, L. See stria'tum, L.

Ge'um, L. Rosi'CE.x, Roseworts. Icos. Pol.

intermed'ium, Ehrh. 46. p. 7. Intermediate Avens.

riva'le, L. 90. p. 5-7. Water Avens.

urba'num, L. 104. p. 6-8. Herb Bennet. Wood Avens.

\begin{tabular}{|l|} 
L. C. \\
322 \\
$322 \mathrm{~b}$
\end{tabular}

Githa'go, Desf. seg'etum, Desf. = Lych'nis Githa'go, Scop.

Glad'iolus, Tourn. IniD'E A, Irids. 'Tri. Mon.

communis, L. = G. illyr'icus, Koch.

illyr'icus, ${ }^{6}$ Koch. 1. p. 6, 7. Lesser Gladiolus. Sword Flag.

Glau'cium, Tourn. Papa'rera'ces, Poppyworts. Polyan. Mon. corni'cula'tum, Curt. = G. phœni'ceum, Crantz.

fla'vum, ${ }^{7}$ Crantz. 50. b. or p.6-10. Yellow Horned Poppy. Sea P. lu'teum, Scop. $=$ G. fla'vum, Crantz.

phoni'ceum, ${ }^{9}$ Crantz. a. 6, 7. Scarlet Horned Poppy.

viola'ceum, Juss. = Rœme'ria hyb'rida, DC.

Glaux', 'Tourn. Prísula'ces, Primworts. Pent. Mon. marit'ima, L. p. 6, 7. Black Saltwort. Sea Milkwort.

Glecho'ma, L.

hèdera'cea, L. = Nep'eta Glecho'ma, Benth.
976

$976 \mathrm{~b}$

978

977

975

973

974

965

291

302

301

303

$303 \mathrm{~b}$

298

293

294

296

299

297

304

$304 b$

$304 \mathrm{c}$

300

291

$29 \mathrm{lb}$

$291 \mathrm{c}$

292

295

ii.296

ii. 301

ii. 293

ii. $293, \beta$

ii. 300

ii. 298

ii.305

ii. 306

200

479

iii. 458

480

iii.459

478

iii. 457

304

303

B.

227

677

678

676

674

675

211

210

206

207

201

203

208

204

205

209$$
\text { . }
$$

202

1379 ix. 1493

1014

(1014)

i. 65

(i.66)

i. 66

(i.65)

(44)

44

60

955

vii. 1150

656
1 Felwort.

5 Geranium prostratum.

8 Squats.
2 Geraninm Raii.

3 Geraninm perenne.

6 Glarliolns commnnis. 9 Gluncinm corniculatum.
4 Perennial Dove's-foot Cranesbill. 7 Glancium luteum. - Chelidonium Gluucinm. 
Glyc'e, Lindl.

marit'ima, Lindl. = Alyss'um marit'imum, L.

Glycer'ia, R.Br. Gramin' ex, Grasses. Tri. Dig.

aqua'tica, ${ }^{1}$ Sm. 76. p. 7,8. Reed Meadow-grass or Sweet-grass. Borre'ri, ${ }^{2}$ Bab. 12. b. or p. 6-8. Borrer's Meadow-grass.

dist'ans, ${ }^{3}$ Wahl. 52. b. or p. 7, 8. Reflexed Meadow-grass.

flu'itans, ${ }^{4}$ R.Br. 110 . p. 7, 8. Floating Meadow-grass. Manna-

lŏlia'cea, H. C. Wats. = Festu'ca lŏliä́cea.

[grass.

marit'ima, Wahl. 67. p. 7. Creeping or Sea Meadow-grass.

v. hisp'ida, Parn.

v. ripa'ria, Towns.

plica'ta, Fr. 62. p. 6-8. Folded-leaved Meadow-grass.

r. pedi'cella'ta ('Towns.)

v. de'clina'ta, "Breb." Towns.

procumb'ens, Sm. = Festu'ca procumb'ens, Kunth.

rig'ida, Sm. = Festu'ca rig'ida, Kunth.

Gnaphal'ium, L. Compos'its, Composites. Syn. Sup.

arvens'e, Willd. = Fila'go min'ima, Fr.

dioic'um, L. = Antenná ria dioic'a, R.Br.

r. hyp'erbor'eum = Antenna'ria dioic'a, v. byp'erbor'ea.

gall'icum, Huds. = Fila'go gall'ica, L.

germa'nicum, Sm. = Fila'go germa'nica, $\mathrm{L}$.

In'teo-alb'um, L. C. a. 7, 8. Jersey Cudweed.

margar'ita'ceum, L. = Antenna'ria margar'ita'cea, R.Br.

min'imum, Sm. = Fila'go min'ima, Fr.

norve'gicum, Gunn. 4. p. 8. Highland Cudweed.

rect'um, Sm. = G. sylva'ticum, L.

supi'num, L. 15. p. 7, 8. Dwarf Alpine Cudweed.

sylva'ticum, ${ }^{6}$ L. 97. p. 7-9. Upright $C$. Wood $C$.

üli'gino'sum, L. 107. a. 7-9. Marsh Cudweed.

v. pǔlula're (Wahl.)

Goodye'ra, Br. Orcuin'es, Orehids. Gyn. Mon.

re'pens, ${ }^{7}$ R.Br. 16. p. 6,7. Creeping Lady's-tresses.

Grimin'Es, Juss. Grasses.

Grammi'tis, Sw.

Cet'erach, Sw. = Cet'erach offic'ina'rum, Willd.

leptophyll'a, Sw. = Gymnogramm'e leptophyll'a, Desv.

Gymnade'nia, R.Br.

alb'ida, Rich. = Habena'ria alb'ida, R.Br.

cōno'psea, R.Br. = Habena'ria cōno'psea, Benth.

Gymnogramm'e, Desv. Fil'ices, Ferns. Crypt. Fil. leptophyll'a, ${ }^{8}$ Desv. C. a. or b. 3-5. Annual Maiden Hair. ${ }^{8}$

1714

1717

xi. 1751

1712 xi. 1752

$\begin{aligned} & 1715 \\ & 1715 b\end{aligned} \mid(x i .1754)$

$1715 \mathrm{c}$

1713

xi. 1753

$1713 \mathrm{~b}$

$1713 \mathrm{c}$

.

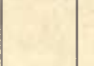

v.747

v. 748

743

v.742

v. $7 \pm 6$

505

745

v.744

746

744

v. 745

v. 743

507

742

v.741

506

$742 \mathrm{~b}$

1335

ix. 1475

1626

xi. 1686

991

1277

ix. 1461

ix. 1460

1805

xii. 1843

$(127 \%$

Hăbena'ria, R. Br. Orchid'es, Orchids. Gyn. Mon. alb'ida, ${ }^{10}$ R.Br. 45. p. 6-8. Small White Orehis.

bifol'ia, ${ }^{11}$ R.Br. 73. p. 6+8. Lesser Butterfly O. White Habenaria. chlōranth'a, Bab. = H. chlōroleu'ca, Ridley.

chlöroleu'ca, ${ }^{12}$ Ridley. 82. p. 5, 6. Greater Butterfly Orehis. cōno'psea, ${ }^{13}$ Benth. 98. p. 6-8. Fragrant $O$. or Gymnadenia. intact'a, ${ }^{14}$ Benth. I. p. 6, 7. Dense-flowered Orehis. vir'idis, ${ }^{15}$ R.Br. 91. p. 6-8. Frog Orchis. Green Habenaria.

Halora'gee, R.Br. Hippurids.

Halosc'ias, Fr. sco'ticum, Fr. = Ligus'ticum sco'ticum, L.

Hed'era, L. Ará'ia'Ces, Ivyworts. Pent. Mon. Hel'ix, L. 111. s. 10,11. Ivy.

2 Poa Borreri.-Sclerochloa Borreri.

(ix. 1461)

1004

1365

ix.1464

1003

1368

1363

ix.1463

(ix. 1463)

1364

(ix. 1460)

1366

(ix.1465)

(995)

ix. 1462

1005

577 iv. 513

1 Pua aquatica.-Hydrochloa aquatica.

iv. 633

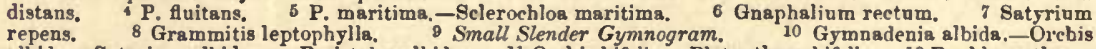
albida, - Satyrium albidum.-Peristylus albidus. 11 Orchis bifolia.-Platanthera bifolia. 12 P. chlorantha.Habenaria chlorantha. is Gymnadenia cunopsea.-Orchis conopsea. It Neotinea intacta.-Orchis intacta. 13 Satyrinm viride,-Peristylns viridis. 
Hedyp'nŏis, Tourn.

autumna'lis, $\mathrm{Sm} .=$ Leont'odōn autumna'lis, $\mathrm{L}$.

hirt'a, Sm. = Leont'odōn hirt'us, L.

hisp'ida, Sm. = Leont'odōn hisp'idus, $\mathrm{L}$.

Tarax'aci, Sm. = Leont'odōn autumna'lis, v. pratens'is.

Hēdys'arum, L.

Un'obrych'is, I. = On'obrych'is sati'va, Lam.

Hel'eoch'aris, Lestib. See El'eoch'aris.

Hel'eogi'tōn, Schult. See El'eogi'ton.

He'lianth'emum, Pers. Cisrix'Es, Rock-roses. Polyan. Mon. āpenni'num, DC. $=$ H. po'ifol'ium, Mill.

Brewe'ri, Planch. 1. a. 7-9. Brewer's Spotted Rock-rose. ca'num, Duval. = H. mar'ifol'ium, Mill.

Chamrecist'us, ${ }^{1}$ Mill. 88. a. 7-9. Rock-rose. Rockcist. gutta'tum, ${ }^{2}$ Mill. C. a. 6-8. Bractless Spotted R. or Sun-rose. le'difol'ium, Willd. a. 6, 7. Ledum-leaved Rock-rose.

mar'ifol'ium, ${ }^{3}$ Mill. 9. s. 5-7. Dwarf Hoary Rock-rose. v. vi'nea'le (Pers.)

pol'ifol'ium, ${ }^{4}$ Mill. 2. s. 5-7. White Mountain R. White Sun Cistus. surreja'num, Mill. s. 7, 8. A garden variety of $\mathrm{H}$. Chamæcist'us. vulga're, Gærtn. $=$ H. Chamæcist'us, Mill.

Hell'eborus, L. Raxuxc'ula'cex, Crmofoots. Polyan. Pol. foe'tidus, L. 13. p. 2, 3. Stinking Hellebore. Setterwort. vir'idis, L. 28. p. 3, 4. Grecn Hellebore.

Helminth'ia, Juss. ech'ivï'des, Gærtn. = Pi'cris ech'ioï'des, $\mathrm{L}$.

Hĕlosc'iadi'um, Koch.

inunda'tum, Koch. = Ap'ium inunda'tum, Reichb.

no'diflo'rum, Bab. = Ap'ium no'diflo'rum, Reichb.

r. re'pens, Koch. = A. no'diflo'rum, v. re'pens.

re'pens, Koch. = Ap'ium no'ditlo'rum, v. re'pens.

He'racle'um, L. UmbelliF' era, Umbellifers. Pent. Dig. Sphondyl'ium, L. 110. p. 6-8. Cow-Parsnip. Hog-weed.

Hermínium, R.Br. URCHId'Ex, Orchids. Gyn. Mon. Monorch'is, ${ }^{5}$ R.Br. 22. p. 6-7. Green Musk Orchis.

Hernia'ria, Tourn. Ill'́tembra'ces, Knotworts. Pent. Dig. cil'ia'ta, Bab. 1. p. 7, 8. Hairy Rupture-wort.

gla'bra, I. 4. a. or b. 7. Smooth Rupture-wort.

v. sub'cilia'ta, Bab.

hirsu'ta, L. 1. p. 7, 8 .

Hesp'eris, L. Crecif'ere, Crucifers. Tetrad. Siliq. inodo'ra, L. $=\mathrm{H}$. ma'trona'lis, $\mathrm{L}$.

ma'trona'lis, ${ }^{6}$ L. p. 5-7. Dame's Rocket. Dame's Violet.

Hiera'cium, L. Cosros'in. Composites. Syn. Eq. aggrega tum, Backh. 2. p. 7, 8. Compact Hawkweed. alpi'num, I. $=$ H. hol'oseric'eum, Backh.

amplex'icau'le, L. p. 7, 8. Anplexicaul-leaved Hawkweed.

an' glicum, ${ }^{7}$ Fr. 18, p. 7, 8. English Hawkweed.

v. ceriuth'iform'e, Backh. in litt.

v. amplex'icau'le, ${ }^{8}$ Bab.

v. acu'tifol'ium, Backh.

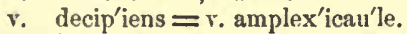

argent'eum, Fr. 12. p. 7, 8. Silvery Hawkweed.

aurant'iacum I. p. 6. 7. Orange Hawkweed.

Auric'ula, I. p. 7.

borea'le, ${ }^{9}$ Fr. 87. p. 8-10. Shrubby Broad-leaved H. Savoy $H$.

Borre'ri, Syme. $=$ H. jura'num, Fr.

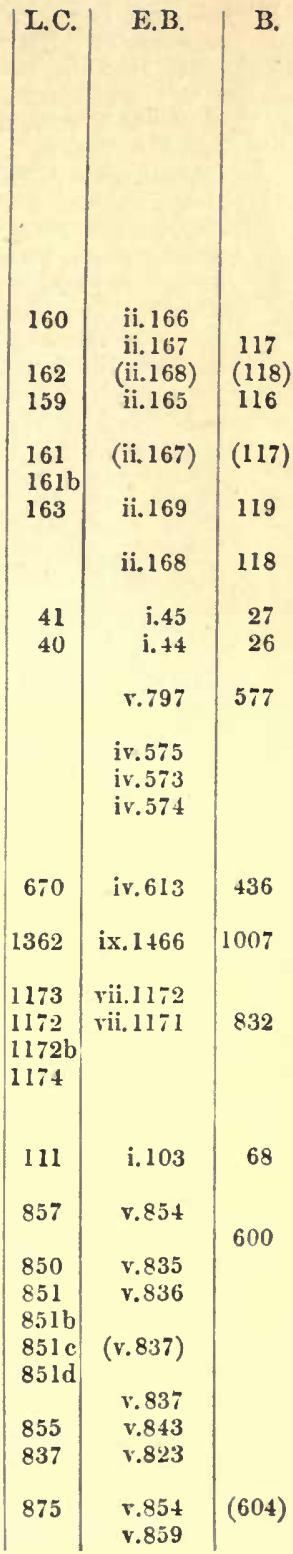

B. 
Hiera'cium, L.-continued.

cx'sium, Fr. 21. p. 6-8. Casion's $H$. Glaucous $H$.

v. Smith'ii, Baker.

v. camb'ricum, Baker.

calend'uliflo'rum, Backh. 4. p. 7, 8. Marigold Hawkwced. cerinth'oïdes, L. p. 7, 8. Honeywort Hawkweed.

chrysanth'um,' Backh. 10. p. 7, 8. Golden-flowered $H$.

v. mi'croceph'alum, Backh.

cinerasc'ens, Jord. $=$ H. pall'idum, v, crinig'erum.

corymbo'sum, Fr. = H. Eupator'ium, Griseb.

croca'tum, ${ }^{2}$ Fr. 27. p. 7-9. Saffron-colonured Hawkweed.

dent'icula'tum, Sm, $=$ H. strict'um, Auct. Angl.

Dewa'ri, Bosw. 3. p. 8, 9. Dewar's Hawkweed.

dub'ium, L. 1. p. 7 .

Eupator'ium, ${ }^{3}$ Griseb. 21. p. 7-9. Corymbose Hawkwced. exim'ium," Backh. 6. p. 7, 8. Gray-headed Hawkweed. v. tenell'um, Backh.

floccnlo'sum, ${ }^{6}$ Backh. 3. p. 10. Stellately-downy Hawkweed. Gibso'ni, ${ }^{6}$ Backh. 1. p. 7, 8. Cat's-ear Hawkweed. gloho'sum, Backh. 3. p. 7, 8. Globose-headed Hawkweed. go'thicum, Fr. 17. p. 7-9. Naked-headed Hawkweed.

v. la'tifol'ium, Backh.

grac'ilent'um, Backh. 3. p. 7, 8. Slender Hawkwced.

Halle'ri, Vill. $=$ H. exim'ium, Backh.

hol'oseric'eum, ${ }^{7}$ Backh. 8. p. 7, 8. Woolly-headed H. Alpine $H$.

hyp'ochœ'roï'des, Gibs. = H. Gibso'ni, Backh.

in'uloï'des, 'Tausch. $=$ H. croca'tum, Fr.

i'ricum, Fr. 11. p. 7, 8. Irish Hawkweed.

jura'num, ${ }^{8}$ Fr. 2. p. 7, 8 .

las'iophyll'um, Backh. = H. pall'idum, v, crinig'erum.

Lawso'ni, Sm. = H. an'glicum, Fr.

lingula'tum, Backh. 5. p. 7, 8. Lingulate-leaved $H$. macula'tum, Sm. p. 10. Spotted Hawkweed.

mel'anoceph'alum, Tausch. 3. p. 7, 8. Alpine H. Grey-headed H. moll'e, Jacq. = Cre'pis hiera'cioï'des, Wald. \& Kit.

muro'rum, L. pt. 24. p. 7-9. Broad-leaved Wall Hawkweed.

v. crassiusc'ulum, Almq.

v. basifol'ium, Almq.

nigresc'ens, Willd. 7. p. 8, 9. Black-headed Hawkweed. ${ }^{9}$

nit'idum, Backh. 2. p. 6, 7. Scaly-stalked Hawkweed.

norve'gicum, Fr. 1.

obtu'sifol'ium, Backh. 1.

pall'idum, Biv. 36. p. 7,8. Pale Hawkweed.

v. Schmidt'ii, Tausch.

v. crinig'erum, ${ }^{10} \mathrm{Fr}$. 7. Hairy-leaved $H$.

paludo'sum, L. = Cre'pis paludo'sa, Moench.

Prlosell'a, L. 110. p. 5-8. Mouse-ear Hawkweed.

v. Pel'eteria'num, Mer.

pratens'e, Tausch. p. 6, 7.

prenanth'oï'des, Vill. 15. p. 8, 9. Rongh-leaved Hawkweed.

pulmona'rium, Sm. = H. chrysanth'um, Backh.

rig'idum, Hartm. p. 7, 8. Rigid-stemmed Hawkweed.

sabau'dum, L. $=$ H. borea'le, Fr.

saxif'ragum, Fr. 2 or 3.

senesc'ens, Backh. 5. p. 7, 8. Grey Lingulate-leaved $H$.

stellig'erum, Backh. = H. flocculo'sum, Backh.

strict'um, ${ }^{13}$ auct. angl. 15. p. 7, 8. Straight-branehed $H$.

sylva'ticum, Sm. = H. vulga'tum, Fr.

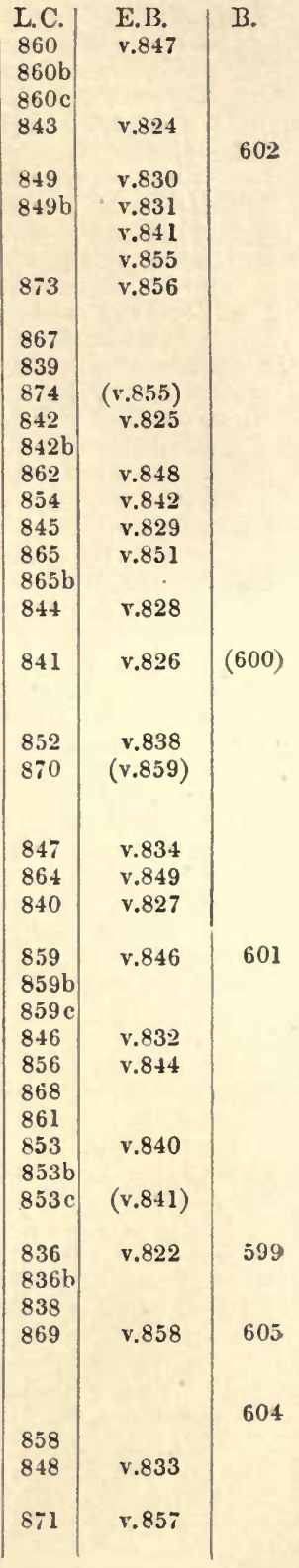

1 H. pulmonarium.

$2 \mathrm{H}$. inuloides.

3 H. corymbosum.

4 H. Halleri.

5 H. stelligerum. 6 H. hypochœroides. 7 H. alpinum. 8 Hjeracium Borreri. 9 Rough-bordered Haukweed. 10 Hierscium cinerascens, - H. lasiophyllum. $\quad$ il H. denticulatum. 
Hiera'cium, L. - continued.

tridenta'tum, Fr. 40, p. 7, 8. Three-toothed Hawkweed.'

umbella'tum, L. 71. p. 7-9. Narrow-leaved $H$.

v. f'lifol'ium, Backh.

villo'sum, L. p. 7, 8. Shaggy Hawkweed.

vulga'tum, ${ }^{2}$ Fr. 55. p. 7-9. Wood Hawkweed.

v. ciner'eum, Backh.

v. rubesc'ens, Backh.

v. macula'tum, anct.

v. nem'oro'sum, Backh.

v. ma'croceph'alum, Backh.

Hieroch'loe, Gmel. GräMn'Ex, Grasses. Tri. Dig. borea'lis, Rnem. \& Schult. 2. p. 5, 6. Northern Holy-grass.

Hippocre'pis, L. Legu'Mino's.e, Leguminous Plants. Diad. Dec. como'sa, L. 45. p. 5-8. Tufted Horse-shoe Vetch.

Hippoph'aë, L. ĖLi'A ANA'CEx, Oleasters. Diœc. Tet. Rhamnoi'des, L. 7. s. 5-7. Sea Buekthorn. Sallow-thorn.

Hippu'ris, L. Halora'Ges, Hippurids. Monan. Mon. vulga'ris, L. 86. p. 6, 7. Mare's-tail.

Hirc'ulus, Haw. ranunc'uloï'des, Haw. = Saxifrraga Hirc'ulus, L.

Holc'us, L. Grāus'e.e, Grasses. Tri. Dig. avēna'ceus, Scop. $=$ Ar'rhenathe'rum avēna'ceum, Beauv. luna'tus, L. 111. p. 6-8. Yorkshire Fog. Meadow Soft-grass. moll'is, L. 103. p. 6-8. Creeping Soft-grass.

Hol'oschœ'nus, Link. vulga'ris, Liuk = Scirp'us Hol'oschœ'nus, L.

Holost'eum, L. umbella'tum, L. a. 4, 5. Umbelliferous Mouse-ear Chickweed. ${ }^{3}$

Honke'nya, Ehrh. pep'loï'des, Ehrh. = Ar'êna'ria pep'loï'des, L.

Hor'deum, L. Grīun'ex, Grasses. Tri. Dig. marit'imum, With. 26.b. 6. Sea-side Barley. Squirrel-tail Grass. 1759 muri'num, L. 75. b.or a.6, 7. Way Bennet. Mouse Barley. Wall B. v. ar'èna'rium, Bab. pratens'e,Huds. [sec'ali'num, Schreb.] 60.a. or p.6, 7. Meadow B. sec'ali'num, Schreb. See pratens'e, Huds. sylva'ticum, Huds. 25. p. 6, 7. Wood Barley. Lyme-grass.

Hotto'nia, L. Pri'MUla'ces, Primeworts. Pent. Mon. palust'ris, L. 48. p. 5, 6. Water Violet or Featherfoil.

Hum'ulus, L. URTícs'Ces, Nettleworts. Diœc. Pent. Lup'ulus, L. 82. p. 7, 8. Common Hop.

Hutchins'ia, R.Br. CrucrF'ERs, Crucifers. Tetrad. Silic. petræ'a, ${ }^{5}$ R.Br. 12. a. 3-5. Rock Hutchinsia.

Hyacinth'us, L.

non-script'us, L. = Scill'a nu'tans, Sm.

rac'emo'sus, $\mathrm{L}$. = Mus'a'ri rac'emo'sum, Mill.

HYDROCH'ARID'E.E, Juss. Hydrocharads.

Hy'drochar'is, L. HYdrocil'ARID'E, Hydrocharads. Diœc. Enn. Mors'us-ra'næ, L. 47. p. 7, 8. Frog-bit.

Hydroch'loa, Hartm. aqua'tica, Hartm. = Glycer'ia aqua'tica, Sm.

Hy'drocot'yle, L. Uniellif'ers, Umbellifers. Pent. Dig. vulga'ris, L. 109. p. 5, 6. Marsh Pennywort. White Rot.

Hym'enophyll'um, Sm. Fı́'ıces, Ferns. Crypt. Fil. ala'tum, Sm. = Trichom'anes, radi'cans, Sw. tunbridgens'e, Sm. 26. p. 6, 7. Tunbridge Filmy Fern.
L.C.

866

E. B.

v.852

v. 853

$872 \mathrm{~b}$

863

v.839

$863 \mathrm{~b}$

$863 \mathrm{c}$

$863 \mathrm{~d}$

$863 \mathrm{e}$

$863 \mathrm{f}$

1641

xi. 1695

1159

379

iii. 380

274

1241

viii. 1245

577

iv. 516

879

394

1678

xi. 1744

1194

xi. 1743

1195

210

ii. 216

157

ii. 239

xi. 1823

1204

xi. 1822

1203

$1758 \mathrm{~b}$

1757

xi. 1821

1202

1756

xi. 1820

1201

940

vii. 1128

646

1265

viii. 1284

904

150

i. 151

100

1323

ix. 1444

1324

ix. 1444

974

610

iv. 566

395

176 
Hym'enophyll'um, Sm.-continued.

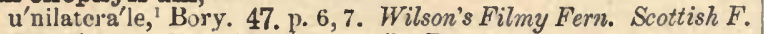
Wilso'ni, Hook. $=$ H. u'nilatera'le, Bory.

Hyosc'yamus, L. So'LAxa'ces, Nightshades. Pent. Mon. nig'er, L. 78. b. or a. 6-8. Conmon Henbane.

Hyos'eris, I.

min'ima, L. = Arnos'eris pusill'a, Gærtn.

HYP'ERICIN'EX, Tutsans.

Hy̆per'icum, L. HxP'ericin'ese, Tutsans. Polyad. Pol. Androsæ mum, ${ }^{2}$ L. 74. s. 6-8. Wild Tutsan.

an'glicum, Bert. $=$ H. ela'tum, Ait.

barba'tum, Jacq. p. 9, 10. Bearded St. John's Wort.

boe'ticum, Boiss. = H. undula'tum, Schousb.

calyci'num,L.s.7-9. Large-flowered St.John's Wort. Aaron's Beard. dub'ium, Lecrs. = H. quadran'gulum, v. dub'ium.

ela'tum, ${ }^{3}$ Ait. s. 7. Tall St. John's Wort.

elo'des, L. 60. p. 7, 8. Marsh St. John's Wort.

hircinum, L. s. 7, 8. Stinking St. John's Wort.

hirsu'tum, I. 86. p. 7, 8. Hairy St. John's Wort.

hŭmifu'sum, L. 95. p. 7, 8. Trailing St. John's Wort.

v. decumb'ens, Petermann.

lina'rixefol'ium, Vahl. 3. p. 6, 7. Flax-leaved St. John's Wort. ${ }^{4}$ monta'num, I. 44. p. 7, 8. Mountain St. John's Wort.

perfora'tum,L. 97.p.7-9. Dotted-leaved or Perforated St.John's W.

v. angust'ifol'ium, Gaud.

pulch'rum, L. 111.p.6,7. Small Upright or Slender St. John'sW. quadran'gulum, L. (ex p.) Fr. 71.

v. dub'ium ${ }^{5}$ (Leers.) Imperforate St. John's Wort.

v. macula'tum, Bab.

quadra'tum, ${ }^{6}$ Stokes. 100 . p. 7-9. Square-stemmed St.John's $W^{7}$ tetrapt'erum, Fr. = H. quadra'tum, Stokes.

undula'tum, ${ }^{8}$ Schousb. 4. p. 8,9. Waved-leaved St. John's W.

Hy̆pochœ'ris, L. Compos'its, Composites. Syn. Aiq.

gla'bra, I. 36. a. 6-8. Smooth Cat's-ear.

v. Balbi'sii (Lois.)

macula'ta, L. 7. p. 7, 8. Spotted Cat's-ear.

ra'dica'ta, I. 107. p. or b. 6-9. Long-rooted Cat's-car.

Hy̆popit'y̌s, Dill. Mox'otrop'Ex, Fir-Rapes. Dec. Mon.

Monot'ropa, Crantz. See multiflo'ra, Scop.
multiflo'ra, ${ }^{9}$ Scop. [Monot'ropa, Crantz.]

Ibe'ris, I. Crucif'ere, Crucifers. Tetrad. Silic. ama'ra, L. 14. a. 7, 8. Bitter Candytuft.

nu'dicau'lis, $\mathbf{L} .=$ Teesda'lia nu'dicau'lis, R.Br.

I'lex, I. I'LICIN'Es, Hollyworts. Tetran. Tetrag.

Aq'uifol'ium, L. 101. t. 5-8. The Holly.

I'LICIN'EA, Hollyworts.

ILL'ECEBRA'CEE, Knotworts.

IIl'ecebrum, I. Ill'Ecebra'ces, Knotworts. Pent. Mon. vert'icilla'tum, I. 4. a. 7, 8. Whorled Knotgrass.

Impat'iens, L. Geran'is'ces, Cranesbills. Pent. Mou. fulv'a, Nuttall. 7. a. 6-8. Orange Bulsam.

Noli'-mē-tang'ere, I. 24. a. 7-9. Yellow Balsam. Touch-me-not. parviflo'ra, DC. a. 7-11. Small Balsam.

Imper'ato'ria, $\mathrm{L}$.

Ostru'thium, $\mathbf{L}$. = Peuc'edan'um Ostru'thium, Koch.
L.C.

E.B xii. 1841

B.

I

1020

vi. 936

711

259

ii. 264

259

ii. 264

178

ii. 270 bis.

ii. 267

ii. 269

ii. 265

261

272

260

270

267

$267 \mathrm{~b}$

268

271

263

$263 \mathrm{~b}$

269

264

$264 \mathrm{a}$

$264 \mathrm{~b}$

265

266

876

$876 \mathrm{~b}$

878

877

ii. 276

ii. 266

ii. 274

ii. 271

ii. 272

ii. 275

ii. 268

ii. 273

184

181

(ii.269)

(180)

(ii.270)

ii. 270

(ii. 270 bis.)

ห.789

582

v. 791

584

v. 790

583

933

(vi.901)

148

i. 149

99

ii. 316

221

314

1171

1171

ii.316

vii. 117

vii. 1173

833

312

311

313

ii. 314

ii. 313

ii. 315

177

187

185

182

183

186 179 ) 等 
In'ula, L. Conpositre, Composites. Syn. Sup.

Cony'za, ${ }^{1}$ DC. 58, b. 7-9. Ploughman's Spikenard. Rigid Inula. cri'thmoï'des, ${ }^{2}$ L. 19. p. 7, 8. Golden Samphire. ${ }^{3}$

dys'enter'ica, L. = Pulica' ria dys'enter'ica, Gærtn.

Helen'ium, L. p. 7, 8. Elecampane.

Pu'lica'ria, $\mathrm{L} .=$ Pu'lica'ria vulga'ris, Gærtn.

salici'na, I. I. p. 7, 8. Willow-leaved Inula.

IRID'E.E, Juss. Irids.

I'ris, L. IRID'Es, Irids. Tri. Mon.

\section{fotidiss'ima, L. 46. p. 5-7. Fetid Iris. Roast-beef-plant.} v. citri'na, Syme.

Pseud-ac'orus, L. 112. p. 5-8. Yellow Water-Iris or Water-flag. ${ }^{4}$ v. ac'oriform' is (Bor.)

v. Bastard'i (Bol')

tubero'sa, L. p. 4. Tubcrous Iris. Snake's-head Iris.

L.C.

1370

I'satis, L. CrDcri'ers, Crucifers. Tetrad. Silic. tincto'ria, L. b. 7, 8. Dyer's Woad.

Isnard'ia, I.

palust'ris, L. = Ludwig'ia palust'ris, Elliott.

I'soetes, I. Sela'Ginelda'Cex. Crypt. Fil.

Duriæ'i, Hook. = I. Hy'strix, Dur.

echi'nospor'a, Dur. 6.p.7-9 (?). Prickly-spored Lake Quillwort. Hy'strix, ${ }^{5}$ Dur.

lacust'ris, I. 22. p. 5-7. Lake Quillwort. European $Q$.

v. Moore'i, Syme.

Isol'epis, R.Br.

fu'itans, R.Br. = Scirp'us flu'itans, L.

Hol'oschœ'nus, Roem. \& Schult. = Scirp'us Hol'oschœ'nus, L.

Sa'vii, Hook. = Scirp'us Sa'vii, Seb. \& Maur.

Ix'ia, L. seta'cea, R.Br. = Scirp'us seta'ceus, L.

Bulboco'dium, L.= Romul'ea Column'æ, Seb. \& Manr.

Jasióne, L. Campa'nula'Cex, Bellworts. Pent. Mon.

monta'na, L. 78. a.6-9. Annual Sheep's-bit. Sheep's Scabious.

Junca'ce. Juss. Rushes.

Junc'us, L. Juxca'Cex, Rushes. Hex. Mon. acu'tiflo'rus, ${ }^{6}$ Ehrh. 106. p. 6-8. Sharp-flowered Jointed Rush. ${ }^{7}$

v. multiflo'rus, Weihe.

acu'tus, L. 14. p. 7, 8. Greater Sharp Sea-side Rush.

alpi'nus, Vill.

art'icula'tus, $\mathrm{L}_{.}=J_{\text {. acu'tiflo'rus, Ehrh. }}$

atra'tus, Krock.

balt'icus, Willd. 12. p. 7. Baltic or Northern Rush.

biglu'mis, L. 3. p. 7, 8. Two-flowered Rush.

bufónius, L. 112. p. 6-8. Toad Rush.

v. fasc'icula'tus, Koch.

bullbo'sus, L.

campest'ris, Sm. = Lu'zula campest'ris, DC.

cap'ita'tus, Weigel. 1. a. 5-7. Capitate or Dense-headed Rush. 1447

castan'eus, Sm. 6. p. 7,8. Clustered Alpine R. Chestnut R. 1444

cœeno'sus, Bich. = J. Gerard'i, Lois.

commu'nis, E. Mey.

compress'us, Jacq. p. 7, 8. Round-fruited Rush.

con'glomera'tus, L. 110. p. 6, 7. Common Rush.

diffu'sus, Hoppe. 30. p. 7, 8. Diffuse or Loose-flowered Rush. effu'sus, L. 110. p. 7, 8. Soft Rush.

f'liform'is, L. 3. p. 7, 8. Thread Rush. Slender Rush.
151

1830

1831

1829

$1829 \mathrm{~b}$
748

750

747

749

1370

$1370 \mathrm{~b}$

1371

$137 \mathrm{Ib}$

13710

1372

E.B.

v. 767

v. 769

v.770

v.766

v.751

v. 768

ix. 1491

ix.1494

ix.1495

ix. 1496

i. 161

109

xii. 1827

xii.1828

xii. 1826

xii.18:6*

897

1425

vi. 863

$\mathrm{x} .1547$

x. 1567

$1443 \mathrm{~b}$

1438

x. 1558

(1059)

1067

1059

1431

1445

1425

$1425 \mathrm{~b}$

x. 1564

$\mathrm{x} .1557$

x. 1572

$\mathrm{x} .1573$

x. 1574,5

x. 1571

1065

$\mathrm{x} .1555$

1069

x. $1560,1 \quad 1055$

1428

1436

1434

$1+35$

1432

B.

513

515

511

516

1013

1012

1252

1251

611

1058

1070

1063

x. 1575

x. 1560

x. 1562

$\mathrm{x} .1561$

$\mathrm{x} .1565$ 
Jun'cus, L.-continued.

Forste'ri, Sm. = Lu'zula Forste'ri, DC.

Gerard'i, ${ }^{1}$ Lois. 77. p. 6-8. Mud Rush.

E.B.

B.

Gesne'ri, Sm. = J. ten'uis, Willd.

glau'cus, Ehrh. 89. p. 6-8. Hard Rush.

L.C.
1429

$x .1574$

grac'ilis, Sm. = J. ten'uis, Willd.

lamprocarp'us, Ehrh. 109. p. 7, 8. Shining-fruited Jointed $R$.

v. acu tiflo'rus, Buchenau. A Hybrid.

v. ni'gritell'us ${ }^{2}$ (Don.) Black-headed Jointed Rush.

$\mathrm{x} .1568$

marit'imus, Lam. 51. p. 7, 8. Lesser Sharp Sea Rush.

$x .1569$

ni'gricans, Drej.

ni'gritell'us, Don. = J. lamprocarp'us, v. ni'gritell'us.

obtu'siflo'rus, Ehrh. 36. p. 7, 8. Blunt-flowered Jointed R.

pilo'sus, L. = Lu'zula pilo'sa, Willd.

pygmæ'ns, Rich. 1.p. 5, 6. Pigmy Rush.

spica'tus, L. $=$ Lu'zula spica'ta, DC.

squarro'sus, L. 105. p. 6,7. Heath Rush. Moss R. Goose Corn. subvert'icilla'tus, Wulf. =J. supi'nus, v. subvert'icilla'tus.

supi'nus, ${ }^{3}$ Mœnch. 102. p. 6, 7. Lesser Bog Jointed Rush.4

v. Koch'ii, Bab.

v. flu'itans (Lam.)

v. ūli'gino'sus (Roth.)

v. subvert'icilla'tus ${ }^{5}$ (Wulf.)

sylva'ticus, Huds. = Lu'zula max'ima, DC.

ten'uis, ${ }^{6}$ Willd. 1. p. 6-8. Slender Spreading Rush.

trif'idus, L. 17. p. 7, 8. Three-leaved Rush. Highland $R$.

triglu'mis, L. 21. p. 7, 8. Three-flowered Rush. üliogino'sus, Sibth. = J. supi'nus, Mœnch.

Junip'erus, L. Cosir'er.e, Conifers. Diœc. Monad. commu'nis, L. 73. s. 5, 6. Common Juniper.

nána, Willd. 17. s. 5. Dwarf Alpine Juniper.

Knapp'ia, Sm.

a'grostid'ea, Sm. = Mibo'ra vern'a, Adans.

Knaut'ia, Coult.

arvens'is, Coult. $=$ Scabio'sa arrens'is, $\mathrm{L}$.

Kobre'sia, Willl. Crr'ERA'ce.E, Sedges. Monœc. Trian. ca'rici'na, ${ }^{7}$ Willd. 4. p. 8. Sedge-like Kobresia.

Koele'ria, Pers. Grämines, Grasses. 'Tri. Dig. crista'ta, ${ }^{8}$ Pers. 81. p. 6, 7. Crested Hair-grass.

v. grac'ilis (Boreau)

v. ar'ēna'ria, Lej.

Ko'niga, R.Br:

marit'ima, R.Br. = Alyss'um marit'imum, $\mathbf{L}$.

LA $\boldsymbol{B}^{\prime} \mathbf{A}^{\prime} \mathbf{T} \boldsymbol{x}$, Juss. Labiates.

Lactu'ca, L. Compos'rte, Composites. Syn. AEq. alpi'na, ${ }^{\theta}$ Benth. 2. p. 7. Alpine Sow-thistle. Blue S. muralis, ${ }^{10}$ Fresen. 67. p. 6-8. Ivy-leaved Wall Lettuce. salig'na, L. 9. b. 7, 8. Least Lettuce. Willow Lettuce. Scáriola, L. 6. b. 7, 8. Prickly Lettuce. viro'sa, L. 51. b. 7, 8. Strong-seented Lettuce. Wild L. Acrid L. u'rus, L. Grämin'ex, Grasses. Tri. Dig. ova'tus, I. C. a. 6. Ovate Hare's-tail-grass.

La'mium, L. LAB' IA'тx, Labiates. Did. Gym. alb'um, L. 100. p. 5-12. White Dead-nettle. White Arehangel. 1153 amplex'icau'le, L. 91. a. 5-8. Hen-bit Dead-nettle. Great H. 1148 
La'mium, L. - continued.

Găleob'dolon, ${ }^{1}$ Crantz. 61.p.5,6. Fellow Archangel. Weazel Snout.

inci'sum, Willd. = L. hyb'ridum, Vill.

intermed'ium, Fr. 29, a. 6-9. Intermediate D. Scotch $D$. macula'tum, L. p. 6-9. Spotted Dead-nettle.

purpur'eum, L. 110, a. 4-10. Red Dead-nettle or Archangel.

r. decip'iens, Sonder.

vulga'tum =L. macula'tum, L. \& L. alb'um, L.

Lamprotham'nus. Chars'ce.s, Charads.

alo'pecu'roi'des, ${ }^{3}$ A.Br. 1. p. 6,7. Foxtail Chara.

Lapp'a, Tourn.

gla'bra, Lam. = Arct'ium ma'jus, Schk.

to'mento'sa, All. = Arct'ium ma'jus, v. sub'tomento'sa.

Lapsa'na, L. Compos'ıx e, Composites. Syn. Aq. commu'nis, L. 110. a. 7-9. Nippleucort.

pusill'a, Willd. = Arnos'eris pusill'a, Gartn.

Lar'brea, St. Hil.

aqua'tica, St. Hil. = Stella'ria üli'gino'sa, Murr.

Lastræ'a, Presl. Fil'ices, Ferns. Crypt. Fil.

a'mula, ${ }^{4}$ Brackenbridge. 34. p. 8, 9. Hay-scented Fern.

crista'ta, ${ }^{5}$ Presl. 9. p. 8. Crested Shield-fern.

di'lata'ta, ${ }^{6}$ Presl. 76.p.8,9. BroadShicld-fern. Prickly-toothedFern.

r. tan'acétifol'ia, Moore.

v. du'meto'rum, Moore.

r. colli'na, Bab.

v. alpi'na, Moore.

v. lepido'ta, Moore.

Fil'ix-mas, ${ }^{7}$ Presl. 112. p. 6, 7. Male Fern. Blunt Shield-fern.

v. affi'nis, Bab.

r. pal'ea'cea, Moore.

v. pu'mila, Moore.

v. abbrev'ja'ta, Bab.

Fœ'nisec'ii, Wats. = L. æ'mula, Brackenbridge.

glandnlo'sa, Newm. p. 8, 9. Bennett's Shield-ferm.

monta'na, Moore $=\mathrm{L}$. Oreopt'eris, Presl.

Oreopt'eris, ${ }^{8}$ Presl. 97. p. 7. Sweet Mountain Buckler-firm. ${ }^{9}$ remo'ta, ${ }^{10}$ Moorc. p. 8, 9. Remote Shield-fern.

rig'ida," Presl. 6. p. 7, 8. Rigid Shield-fern.

spi'nulo'sa, ${ }^{12}$ Presl. 83.p.8,9. Narrow Prickly-toothed Shield-fern.

v. élera'ta, A.Br.

v. exalta'ta (Lasch ex p.)

v. decip'iens, Syme.

v. glandulo'sa (Moore ex p.)

Thèlypt'eris, ${ }^{13}$ Presl. 41.p.7,8. Marsh Fern. Fe male Buckler-fern. üli'gino'sa, ${ }^{1+}$ Newm, 6. p.9. Lloyd's Shield-fern.

Lathræ'a, L. OR'овахсHA'CEx, Broomrapes. Did. Ang. squama'ria, L. 62. p. 4, 5. Greater Toothwort.

Lath'yrus, L. LEGu'Mixo'sx, Leguminous Plants. Diad. Dec. Aph'aca, L. 27. a. 6, 7. Yellow Vetchling.

hirsu'tus, L. 2. a. 6, 7. Hairy Vetchling. Rough Podded Vetch. la'tifol'ius, L. p. 7. 8. Broad-leaved Everlasting Pea.

ma'crorrhi'zus, ${ }^{15}$ Wimm. 99. p. 6-8 Tuberous Bitter V'etch. ${ }^{16}$

v. ten'uifol'ius (Roth)
I.C.

1154

1150

1149

1152

1151

$1151 \mathrm{~b}$

1847

(xii.1909)

E.B.

vii. 1087

(vii.1083)

vii. 1083

vii. 1082

vii. 1085

rii.1084

810

808

825

v.787

608

1800

1796

1799

$1799 \mathrm{~b}$

$1799 \mathrm{c}$

$1799 \mathrm{~d}$

$1799 \mathrm{e}$

$1799 \mathrm{f}$

1793 xii.1850

(1282)

$1793 \mathrm{~b}$

$1793 \mathrm{c}$

$1793 \mathrm{~d}$

$1793 \mathrm{e}$

1792

1795

1794

1798

$1798 \mathrm{a}$

$1798 \mathrm{~b}$

$1798 \mathrm{c}$

$1798 d$

1791

1797

1091

395

397

400

405

$405 \mathrm{~b}$

xii.1856

xii. 1849

(1281)

xii.1852

xii.1851

xii.1855

xii. 1848

(1280)

xii.1854

vi. 1006

722

iii. 397

iii. 399

287

iii. 403

iii.406

288

294
I Galeobdolon lateum. Nephrodium amulum.

Nephrodiam dilatatum.

Nephrodium Oreopteris,-L

rodium remotam.

Nephrodium spinulosum.

14 Tephrodium aliginosum.
4 Lastra Fanisecii.-

2 Laminm incisum.

3 Chara alopecuroidea.

5 Aspidium cristat um.- Nephrodium uristatam.

7 AspidinmFilix-mss, - Nephrodinm Filix-mas.

11 Aspidium rigidnm.-Nephrodium rigidum.

$\theta$ Heath

6 Aspidium dilatatum.-

8 Aspidinm Orenpteris. 12 Aspidium spinulosum. 13 Aspidin m Thelypteris. - Nephrodium Thelypteris.-Polypodium Thelspteris. 15 Orobus tuberosus. 16 Ileath or Eurthnut Pea. 
Lath'yrus, L-continued.

marit'inus, ${ }^{1}$ Bigel. 9. p. 6-8. Sea-side Everlasting Pea. v. acu'tifol'ius, Bab.

nig'er, ${ }^{2}$ Wimm. 4. p. 6-8. Blaek Pea or Bitter Vetch.

Nisso'lia, I. 40. a. 5, 6. Grass-leaved Vetchling. Grass Vetch. palust'ris, L. 19.p. 6-8. Blue Marsh Vetchling or Pea. Luckling V. pratens'is, L. 109. p. 6-9. Yellow Mcadow Vetchling or Pea. sphe'ricus, Retz. a. 6.

sylvest'ris, L. 60. p. 6-8. Narrow-leaved Everlasting Pea. tubero'sus, L. p. 6-8. Tuberous Vetehling. Crimson Pea.

Lavate'ra, L. Malra'CEx, Mallow worts. Monad. Pol.

urbor'ea, L. 13. b. 7-9. Sea Tree Mallow.

olb'ia, s. 8. Tree Lavatera.

sylvest'ris, Brot. 1. a. or b. $6,7$.

Le'dum, L. ER'íca'cex, Heathworts. Dec. Mon. palust're, L. s. 7, 8. Marsh Cistus.

Leers'ia, Sol. Grämin'es, Grasses. Tri. Dig.

ory'zoi'des, Sw. 3. p. 8.10. European Cut-grass.

LeGu'mixo's.e, Juss. Leguminous Plants.

Leiog'ynē, Don. (Lindl. Syn.)

a' zoï'des = Saxi'fraga ai'zoï'des, $\mathbf{L}$.

cern'ua = Saxif'raga cern'ua, $L$.

gra'nula'ta = Saxif'raga gra'nula'ta, L.

niva'lis = Saxif'raga niva'lis, $L$.

ri'vula'ris = Saxif'raga ri'vula'ris, $\mathrm{L}$.

Lem'na, L. Lems'ces, Duckweeds. Dian. Mon.

arrhi'za, L. = Wolff'ia arrhi'za, Wimm.

gibb'a, L. 50. p. 6-9. Gibbous or Thick-ieaved Duckweed.

min'or, L. 105. p. 7. Lesser Duckweed.

polyrrhi'za, ${ }^{3}$ L. 54. p. Greater Duckweed.

trisulc'a, L. 70. p. 6, 7. Ity-leaved Duckweed.

Lema'́ce., Duskweeds.

LENT'IBULA'RiE.e, Butterworts.

Leont'odōn, L. Coupos'rte, Composites. Syn. Æq. autumna'lis, ${ }^{4}$ L. 110. p. 7-9. Autumnal Hawkbit. v. pratens is $^{5}$ (Koch)

hirt'us, ${ }^{6}$ L. 65. b. or p. 7, 8. Hairy Hawkbit. Deficient or Lesser $H$. hisp'idus, ${ }^{7}$ L. 87. p. 6-9. Common Rongh Hawkbit. palust're, Sm. = Tarax'acum offic'îna'le, v. palust're. 'Tarax'acum L. = Tarax'acum offic'ina'le, Web.

Leōnu'rus, L. LAB'1A'те, Labiates. Did. Gym. Card'iaca, L. p. 7-9. Motherwort.

\begin{tabular}{|c|c|c|}
\hline L. C. & $\begin{array}{l}\text { E.B. } \\
\text { iii. } 405\end{array}$ & $\begin{array}{l}\text { B. } \\
293\end{array}$ \\
\hline $\begin{array}{l}40+b \\
406\end{array}$ & iii. 407 & 295 \\
\hline 396 & iii. 398 & 286 \\
\hline 403 & iii. 404 & 29.2 \\
\hline 399 & iii. 400 & 289 \\
\hline $\begin{array}{l}398 \\
40.2\end{array}$ & & \\
\hline 402 & iii. 402 & 291 \\
\hline 401 & iii.401 & 290 \\
\hline 275 & ii. $2 ; 9$ & 193 \\
\hline 276 & & \\
\hline $\begin{array}{r}1635 \\
320\end{array}$ & $\begin{array}{r}\text { xi. } 1686 \\
\text { iii. } 323\end{array}$ & 1151 \\
\hline & ix. 1398 & $9+7$ \\
\hline 1467 & ix. 1396 & 945 \\
\hline 1466 & ix. 1395 & 944 \\
\hline 1468 & ix. 1397 & 946 \\
\hline 1465 & ix. 1394 & 943 \\
\hline 1465 & ix. 1394 & \\
\hline 1092 & vii.1121 & \\
\hline 881 & v.794 & 580 \\
\hline $881 \mathrm{~b}$ & ห.795 & \\
\hline 879 & v.792 & 581 \\
\hline 880 & v. 793 & 579 \\
\hline 1147 & vii. 1080 & 806 \\
\hline 142 & i. 156 & 102 \\
\hline 144 & i. 158 & 104 \\
\hline 139 & i. 153 & 105 \\
\hline 140 & i. 154 & 106 \\
\hline 141 & i. 155 & \\
\hline 43 & i. 157 & 103 \\
\hline
\end{tabular}

Lepid'ium, L. Crucif'ere, Crucifers. Tetrad. Silic. campesi're, ${ }^{8}$ R.Br. $\quad 85$. b. 5-8. Mithridate Pepperwort. ${ }^{9}$ did'ymum, L. = Senebie'ra did'yma, Pers.

Drab'a, L. p. 5, 6. Whitlow Pepperwort. Hoary Cress. het'erosty'lum, Benth. See Smith'ii, v. a'latosty'la, Towns. v. canesc'ens, Gren. \& Godr. See Smith'ii, Hook.

la'tifol'ium, L. 19. p. 7, 8. Broad-leaved Pepperwort or Cress. ${ }^{10}$ petræ' 1 , $\mathrm{L}_{1}=$ Hutchins'ia petræ'a, R.Br.

ru'dera'le, L. 36. a. 5, 6. Narrow-leaved or Rubbish P. or $C$. sati'vum, L. a. 6. Garden Cress.

Smith'ii, Hook. [het'erosty'lum, v. canesc'ens]. Hairy Mithridate Pepperwort or Mustard. ${ }^{11}$

83. p. 6-8. v. a'latosty'la, ${ }^{12}$ Tuwns. [het'erosty'lum, Benth.]

$143 \mathrm{~b}$

945

944

946

580

81

79

06

02

04

05

06

03

1 Pisnm maritimum. 2 Orobns niger. 3 Spirodela polyrrhiza, 4 Apargia antumnalis,-Hedypnois antumnalis - Oporinia antumnalis. 5 Apargia Taraxaci.-Hedypnois Taraxaci. Hedspnois hirtum.-Thrincia hirta. 7 Apargia hispida.-Hedypnois hispidum. 10 Poor Man's Pepper. II Smith's Cress. Smooth-fruited Field Peppestris, 12 Thlaspi hirtum. 
Lepigo'num, Wahl. Cak'yopnyl'́e, Cloveworts. Dec. Trig. mar.gina'tum, ${ }^{1}$ Kioch. 34.b.or p.6-8. Greater Sea Sandwort-Spurrey. mari'uum, Wahl. = L. margina'tum, Koch.

ru'brum, ${ }^{2}$ Fr. 92. a. or b. 6-9. Field Sandwort-Spurrey. rupest're, Kindb. 17. p. 6-8. Rock Sca Sandwort-Spurrey. v. glabresc'ens, Lebel.

rupic'ola, Kindb. $=$ L. rupest're, Kindb.

sali'num, ${ }^{3}$ Fr. 43. Lesser Sea Sandwort-Spurrey.

v. genui'num, auct.

v. med'ium (Fr.)

v. neglect'um (Kindb.)

Leptu'rus, R.Br. GRĀmin'Ex, Grasses. Tri. Dig.

fi'litorm' is, ${ }^{6}$ Trin. 47. p. 7. Sea-side Hard-grass. v. incurva'tus ${ }^{7}$ ('Trin.) Curved Hard-grass. incurva'tus, Trin. = L. fíliform'is, v. incurva'tus.

Leucŏj'um, L. X̂MARYldid'E.e, Amaryllids. Hex. Mon. æsti'rum, L. 7. p. 5. Summer Snowflake. vern'um, L. 2. p. 3, 4. Spring Snowflake.

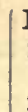

L.C.

248

251
$251 \mathrm{~b}$

1754

$1754 \mathrm{~b}$

1386

1387

Libano'tis, Crantz.

monta'na, All. = Ses'eli Libano'tis, Knch.

Ligust'icum, L. UMBELLIF'ER.E, Umbellifers. P'ent. Dig. corn'ubiens'e, L. = Phys'osperm'um commuta'tum, Spreng. sco'ticum, ${ }^{8}$ L. 23. p. 7. Sea Lovage. Scottish Lovage.

Ligust'rum, Tourn. OL'EA'CEx, Oliveworts. Dian. Mun. vulga're, L. 81. s. 6, 7. Common Privet. Prim. Print.

Li'LIA'CEA, Juss. Lilyworts.

Li'lium, L. Li'LIA'Cex, Lilyworts. Hex. Mnn.

Marta'gon, L. p. 8, 9. Purple Martagon Lily. Turk's Cap L. 1416 pompo'ninm, Bab. =L. pyre'năi'cum, Gouan.

pyre'nđi'cum, ${ }^{9}$ Gouan. p. 7. Yellow Martagon Lily. Pyrenean $L$.

Limbard'a, Cass.

cri'thmoi'des, Cass. = In'ula cri'thmoï'des, L.

tricusp'is, Cass. = In'ula cri'thmoï'des, L.

Limnanth'emum, Gmel. Gentra'see, Gentianworts. Pent. Mon. nymphr'oi'des, Link. = L. pelta'tum, Gmel.

pelta'tum, ${ }^{10} \mathrm{Gmel}$. 10.p.7,8. Round-leaved or Yellow Buckbean. ${ }^{11}$

Li'mosell'a, L. Scro'phucarin'es, Figworts. Did. Ang.

aqua'tica, L. 41. a. 7-9. Common Mudwort.

Lina'ria, Tourn. SCro'Pholarin'Ee, Figworts. Did. Ang.

Cymbala'ria, ${ }^{12}$ Mill. p. 5-9. Ivy-leaved Toadflax or Snapdragon. 1028

Elati'na, ${ }^{13}$ Mill. 55. a.7-10. Sharp-leaved Fluellin or Toadflax. 1029

min'or, Desf. $=$ L. visc'ida, Mœnch.

Pelisse'ria'na, Mill. C. a. 6, 7. Jersey T. Upright-purple T. 1032 purpur'ea, L. b. or p. 8. Purple Tondflax. 1033 re'pens, ${ }^{14}$ Mill. 21. p. 7-9. Striped T. Creeping pale-blue T. 1034 se'pium, ${ }^{15}$ (All.) Hybrid between Yellow and Striped $T$.

spur'ia, Mill. 43.a. 7-10. Round-headed Fluellin. Male F. supi'na, ${ }^{17}$ Desf. a. 7 . Decumbent or Diffuse Toadflax.

visc'ida, ${ }^{15}$ Mænch. 60, a. 5-10. Least Toadflax.

vulga'ri-re'pens $=$ L. se'pium (All.)

vulga'ris, ${ }^{19}$ Mill. 98. p. 7-10. Common Yellow T. Butter \& Eggs. v. la'tifol'ia, Bab.

v. Yelo'ria, p. 8.

L'NEe, DC. Flaxworts.
E.B.

(ii.25\%)

(ii.254)

249

$249 a$

$249 b$

$249 \mathrm{c}$

xi. 1818

(ii. 255)

ix. 1505

ix. 1506

B.

(169)

(1198)

1198

1022

427

668

681

(681)

744

736

738

735

733

732

vi.961

(vi.965)

vi. 957

vi. 958

(vi.966)

vi. 965

vi. 962

vi. 964

vi. 963

286 ii.288
737

734

(735).

731

1 Alsine marina.-Arenaria marina.-A. media.- Lepigonum marinum.-Spergularia marginata.-S. marina. - 8. medis. 2 Arenaria rabra. - Alsine rubra. - Spergnlaria rubra. 3 Red or Purple Sandwort.Sand Spurrey 4 Lepigonn 5 6 Ophiurus incurratus. - Rothoellis incnrvats. 7 Lepturus incarvatus.

9 Liliam pomponium. 10 Limnanthemam nrmphroides, 8 Haloscias scoticum. phreoides. Il Nymphrea-like Villarsia.-Fringed Bog-bean or Water-Lily. J\% Antirrbinum Cymbalaria. 13 A. Elatine. is A. repens. 16 Linsria vulgari-repens. 16 Antirrbinam spurium. 17 A. supinum. 18 A. minus.-Linaria minor. 19 Antirrhinum Linaria. 
Linnæ'a, Gronor. CAr'rifor'ra'ce.e, Caprifoils. Did. Ang. borea'lis, L. 14. p. 7. Two-flowered Linnea. Northern $L$.

Linos'yris, JC. vulga'ris, Cass. = Ast'cr Linos'yris, Bernl.

Ii'num, L. Lr'xes, Flaxworts. Yent. Yentag. angust'ifol'ium, Huns. 35. a. or p. 5-9. Narrow-leaved Pale Flax. cathart'icum, L. 111. a. 6-9. Purging Flax. Mill Mountain. ${ }^{1}$ perenn'e, L. 13. p. 6, 7. Perennial Blue Flax. u'sitatiss'imum, L. a. 6, 7. Common Flax. v. erep'itans, Schüb.

Lip'aris, Rich. Orchin'ex, Orchids. Gyn. Mon.

Luese'lii,'2 Rich. 5. p. 7. Fen Orchis. Two-leaved Bog Orchis.

\begin{tabular}{|c|} 
L.C. \\
687 \\
\\
\\
\\
289 \\
287 \\
288 \\
290 \\
$290 \mathrm{~b}$
\end{tabular}

Iiste'ra, R.Br. Orchiv'Es, Orchids. Gyn. Mon. corda'ta, ${ }^{3}$ R.Br. 54. p. 7-9. Lesser or Heart-leaved Mountain $\mathrm{Ni}^{\prime}$ dus-av'is, Hook. = Neott'ia Ni'dus-ar'is, Rich. [Tway-blade. ora'ta, R.Br. 102. p. 5-7. Common Tway-blade.

IIthosperm'um, Tourn. BorāGIN'Ex, Borageworts. Pent. Mon. arvens'e, L. 86. a. 5, 6. Corn Gromwell. Bastard Alkanet. marit'imum, Lehm. = Mertens'ia marit'ima, Gray. offic'ina'le, L. 77. p. 6, 7. Common Gromwell. Grey Mill. purpur'eo-crerul'eum, L. 8. p. 6, 7. Purple or Creeping $G$.

Littorell'a, Berg. Plantāgin'es, Ribworts. Moncec 'Tet. junc'ea, Berg. See lacust'ris, L. lacust'ris, L. [junc'ea, Berg.] 86. p. 8. Plantain Shoreweed. ${ }^{3}$

Lloyd'ia, Salisb. Lu'LIA'CEE, Lilyworts. Hex. Mon.

alpi'na, ${ }^{6}$ Salisb. 1. p. 6. Monntain Spider-wort or Lloydia. sero'tina, Reich. $=$ L. alpi'na Salisb.

Lobe'lia, L. CaMPa'nula'cex, Bellworts. Yent. Mon. Dortmann'a, L. 36. p. 7, 8. Water Lobelia. u'rens, L. 2. p. 8, 9. Acrid Lobelia.

Loiseleur'ia, Desv. ER'ícA'cex, Heathworts. Pent. Mon. procumb'ens, ${ }^{7}$ Desv. 17. s. 5, 6. Trailing Azalea.

Lol'ium, L. Grāmin'e.e, Grasses. Tri. Dig. arvens'e, With. $=\mathrm{L}$. te'mulent'um, s. arvens'e. ital'icum, Braun. $=$ L. perenn'e, v. ital'icum.

linic' ola, Souder. a. 6, 7. Annual or Flax Rye-grass.

multiflo'rum, Lam. $=\mathrm{L}$. perenn'e, v. multiflo'ra.

perenn'e, L. 112. p. 5, 6. Perennial or Bcardless Rye-grass. ${ }^{8}$

v. remo'tum (Schrank)

v. multiflo'ra ${ }^{9}$ (Lam.)

v. arista'ta, Schum.

$\times$ festūca'ceum (Link)

v. ital'icum ${ }^{10}$ (Braun) Italian Rye-grass.

témulent'um, L. 64. a. 6-8. Annual or Bearded Darnel. v. $\operatorname{arrens}^{\prime} \mathrm{e}^{11}$ (With.)

Loma'ria, Wilid. Fr'jees, Ferns. Crypt. Fil. Spi'cant, ${ }^{12}$ Desv, 111. p. 7, 8. Northern Hard Fcrn. Deer Fern.

Lonice'ra, L. CAP'RIfor'́rA'Cex, Caprifoils. Pent. Mon. Cap'rifol'ium, ${ }^{13}$ L.s.5,6. Pale Perfoliate Woodbine or Honeysuckle. Përiclym'enum, ${ }^{14}$ L. 109.s. 6-9. Common Honeysuckle. Woodbine. Xy̆lost'eum, L. s. 5, 6. Upright Fly Honeysuckle.

Lo'raNtha'CEF, Juss. Loranths.

Lo'tus, L. LEGu'Mino's.e, Lequminons Plants. Diad. Dec. augustiss'imus, ${ }^{16}$ L. 6.a.7,8. Long-podded Small Bird's-foot Trefoil. v. Seringia'nus, Bab.

corni'cula'tus, L. 112. p. 6-9. Common Bird's-foot Trefoil.

r. crassifol'ius, P'ers.

v. villo'sus, Coss. \& Germ.

1327

ix. 1488

E.B.

iv. $6+4$

B.

466

ii.291

ii.289

ii. 290

190

191

ii.292 188

1330

ix.1476

977

1331

ix.1477

985

1005

vii. 1102

984

1004

1003

vii.1101

693

vii. 1100

694

695

1170

vii.1169

830

1420

(ix.1251)

(1034)

ix.1521 1034

895

vi.861

609

896

vi.862

610

925

vi. 884

631

xi.1815

1747

1746

xi.1814

1207

$1746 \mathrm{c}$

$1746 \mathrm{~d}$

$1746 x$

$1746 \mathrm{e}$ (xi.1815)

1748 xi.1816

$1748 \mathrm{~b} \times \mathrm{xi} .1817$

1208

1767

xii. 1885

(1298)

688

iv. 641

iv. 642

464

689

iv. 643

463

690
1242

iv. 635

369

(iii.37l)

265

$369 \mathrm{~b}$

366

iii.368

264

1 Little Flax. 2 Malaxis Loeselii.-Ophrys Loeselii.-Sturmia Loeselii.

$366 \mathrm{c}$

3 Ophrys cordata. 40. orata. 5 Shore-grass. 6 Anthericum serotinum,-Lloydia serotina, 7 Azalea procumbens. 8 Red Darnel. 9 Lolium multiflorum. 10 L. italicum. 11 L. arvense. 12 Blechnum boreale.-B. Spicaut. 13 Caprifolium perfoliatum. 14 C. Periclymenum. 15 Lotus diffusus. 
Lo'tus, L.-continued.

diff'u'sus, $\mathrm{Sm},=\mathrm{L}$. angustiss'imus, $\mathrm{L}$.

hisp'idus, Desf. 6. a. 7, 8. Short-podded or Hairy Small B. T. ma'jor, Sm. = L. pilo'sus, Beeke.

pilo'sus, ${ }^{1}$ Beeke. 100.p.7,8. Greater, Marsh, or Narrow-leaved B.T. ten'uis, Waldst. \& Kit. 63. p. 7. Slender Bird's-foot Trefoil. ũli'gino'sus, Schkuhr. $=\mathrm{L}$. pilo'sus, Beeke.

Ludwig'ia, L. Oxagra'riez, Onagrads. Tetran. Mon. palust'ris, ${ }^{2}$ Elliott. 2. p. 6, 7. Marsh Isnardia.

Lu'zula, DC. JuxcA'Ce. Rushes. Hex. Mon.

arcua'ta, Wahl. 6. p. 7. Curved Alpine Wood-mush.

Borre'ri, Bromf. = L. pilo'sa, v. Burre'ri.

campest'ris, ${ }^{3}$ DC. 105. p. 4-6. Field Wood-mush.

cougest'a, Lej. $=\mathrm{L}$. multifio'ra, v, congest'a.

erect'a, Desv. See multiflo'ra, Lej.

Forste'ri, ${ }^{4}$ DC. 28. p. 4-6. Narrow-leaved Huiry Wood-mush. max'ima, ${ }^{5}$ DC. 105. p. 5, 6. Great Huiry Wood-rush. multiflo'ra, Lej. [erect'a, Desv.] 100. p. 5-8. Many-headed W.

v. congest' $a,{ }^{6}$ Koch.

v. sudética (DC.)

v. pallesc'ens, Koch.

niv'ea, DC.

pilo'sa, ${ }^{7}$ Willd. [verna'lis, DC.] 106. p. 4, 5. Broad-leaved Hairy Wood-rush.

v. Borre'ri, (Bromf.)

spica'ta, ${ }^{9}$ DC. 19. p. 7. Spiked, Hairy, or Mountain Wood-rush. sylva'tica, Bich. = L. max'ima, DC.

verna'lis, DC. See pilo'sa, Willd.

Lych'nis, L. Car'rorhyll'ex, Cloveworts. Dec. Pent.

alb'a, ${ }^{10}$ Mill. 94. p. 6-9. White Campion. Corn Lychnis.

alpi'na, L. 3. p. 6,7. Red Alpine Catchfly. Mountain Campion. dioic'a, Reichb. =L. alb'a, Mill.

diurn'a, ${ }^{11}$ Sibth. 111.p.6,7. Red or Rose Campion. Wood Lychnis.

Flos-cuc'uli, L. 111. p. 5, 6. Ragged Robin. Cuckoo-flower. Smooth $L .{ }^{12}$

Githa'go, ${ }^{13}$ Stop. 97. a. 6-8. Corn Cockle.

sylvest'ris, Hoppe $=\mathrm{L}$. diurn'a, Sibth.

vesperti'na, Sibth. $=\mathrm{L}$. alb'a, Mill.

Visca'ria, L. 10. p. 6-8. Red German Catchfy. Fiscid L. Cuckoo-flower.

L.C.

370

368

367

604

1451

1453

1448

$1+50$

1454

$1454 \mathrm{~b}$

$145+\mathrm{c}$

$1454 d$

1449

$1449 \mathrm{~b}$

1452

$\mathrm{x} .1548$

$\mathrm{x} .1553$

$\mathrm{x} .1549$

204

208

205

206

203

(ii.210)

ii. 214

(ii. 211)

ii. 212

ii. 215

1071

1075

1072 .

(139)

144

140.

142 :

141

139

207

ii. 213

143 ,

Iych'notham'nus, Chara'cex, Charads. Crypt. Char. stell'iger, ${ }^{14}$ A.Br. 3. p. 8, 9. Star-bearing Chara.

Lyc'ium, L. So'lana'ce. Nightshades. Pent. Mon. bar'barum, L. p. 6. Tea-plant.

I,rC'OPOD'1A'CE S, Sw. Club-mosses.

Lyc'opod'ium, L. Lxc'opon'in'CE $E, C l u b$-mosses, Crypt. Fil. alpi'num, L. 55. p. 6-8. Savin-leaved Club-moss. Alpine C. v. decip'iens.

anno'tinum, L. 17. p. 6-8. Interrupted Club-moss.

clava'tum, L. 88. p. 7, 8. Common Club-moss.

complana'tum, L. 6. p. 8 .

v. an'ceps.

inunda'tum, L. 54. p. 6-8. Marsh Club-moss.

sela'ginoï'des, L. = Sela'ginell'a sela'ginoï'des, Gray.

Sela'go, I. 83. p. 6-8. Fir Club-moss.

v. recurv'um, Syme.
1846 (xii.1910)

1017 vi.933

$182.2 \times i i .1830$

1826 xii.1834 1255.

xii. $1834^{*} A$

\begin{tabular}{l|l|l}
1824 & xii.1832 & 1254
\end{tabular}

1825 xii. $1833 \quad 1253$

1827

1823 xii. $1834^{*} B$

1258

1822 xii. $1830 \quad 1256$.
2 Isnardia palustris.

3 Juncus campestris. 6 Isnardia palustris. 3 Juncus campestris. 4 J. Forsteri. $5 \mathrm{~J}$. sylvaticus, - Lozni aliginosus. spicatus. $\quad 10$ Lychnis dinica,-L, respertina. - Silene pratensis, 12 Mendon Pink.-Zose of Heaven.-Wild Williams. 14 Chara stelligera.

1 Lotus major.-L. uliginosus. 
Lycops'is, L. Borāgix'Es, Borageworts. Pent. Mon. arvens is, ${ }^{1}$ L. 99. a. 6, 7. Small Bugloss.

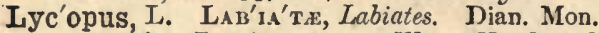
europæ'us, L. 90. p. 6-9. Water Horehound. Gipsywort.

Iy'simach'ia, L. PRi'MULA'CEe, Primworts. Pent. Mon. cil'ia'ta, L. p. 7. Ciliated or Fringed Loosestrife. nem'orum, L. 105. p. 5-7. Yellow Pimpernel. Wood L. Nummula'ria, L. 67. Money-wort. Creeping Jenny. ${ }^{2}$ puncta'ta, L. p. 6, 7. Dotted or Punctate Loosestrife. thyrsiflo'ra, L. 13. p. 6, 7. Tufted Loosestrife.

vulga'ris, L. 78. p. 7, 8. Great Yellow Loosestrife. v. angust'ifol'ia, H. C. Wats.

I ÝTILRÁries, Juss. Loosestrifer.

Ly'thrum, L. LȲThrA'k1Ee, Loosestrifes. Dod. Mon.

Hysso' pifol'ia, I. 6. a. 6-9. Hyssop-leaved Loosestrife. Grass Poly. Sălica'ria, L. 92. p. 7-9. Spiked Purple L. Upright Purple L. v. canesc'ens, Koch.

Maianth'emum, Mœnch. LI'LIA'cex, Lilyworts. Hex. Mon. bitol'ium, DC. $=$ M. Convalla'ria, Web.

Convalla'ria, ${ }^{3}$ Web. 1.p. 5, 6. May Lily. Two-lcaved Smilacina.

$$
\begin{array}{|l}
\mathrm{L} \\
9 \\
11 \\
9 \\
951 \\
95 \\
9 \\
95 \\
9 \\
9 \\
9 \\
587 \\
588 \\
587 \\
58
\end{array}
$$

L.C. F.B.

B.

990 (vii.il1)

703

1116 vii.1019

776

951 vii.1143

953 vii.1145

654

952 vii.1144

653

950 vii.1142

948 vii.1140

652

949 vii.1141

651

587

iv. 491

588

iv.492

354

587 iv.491

353

Malach'ium, Fr. aqua'ticum, Fr. = Stella'ria aqua'tica, Scop.

Malax'is, Sw. Orcind'Es, Orchids. Gyn. Mon. Loese'lii, Sw. = Lip'aris Loese'lii. Rich. paludo'sa, Sw. 43. p. 7-9. Least or Two-leaved Bog-Orchis. ${ }^{4}$

ix.1489

976

Malcol'mia, Br. Crucif'ere, Crucifers. 'Tetrad. Siliq. marit'ima, Br. a. 5, 6. Virginian Stock.

Malv'a, L. Malva'CEs, Mallow-worts. Monad. Pol. horea'lis, Wallm. [ pusill'a, With.] a. 7. Small-flowered Mallow. moscha'ta, L. 85. p. 7, 8. Musk Mallow. parvifo'ra, L. pusill'a, With. See borea'lis, Wallm. rotund'ifol'ia, L. 78. p. 6-9. Dwarf Mallow. sylvest'ris, L. 96. p. 6-9. Common Mallow. vert'icilla'ta, L. 2. 6, 7. Erect Mallow.

Malva'ces, Juss. Mallowworts.

1394 (ix.1510)

(1029)

ii. 283

27 ii. 280

196

28.2

279

ii.282

194

278

ii. 281

280 ii. 284

195

Marrub'ium, L. Labia'te, Labiates. 1)id. Gym.

273

ii. 278 vulga're, L. 65. p. 7-11. White Horehound.

Mars'ileácese, R.Br. Pepperworts.

Maru'ta, Cass. fo'tida, Cass. = Anth'emis Cot'ula, I.

Ma'tricária, L. Compos'ite, Composites. Syn. Sup. Chamomill'a, ${ }^{5}$ L. 62. a. 6-8. Wild Chamomile. in'odo'ra, ${ }^{6}$ L. 111. p. 6-10. Scentless Mayweed. Corn Feverfew. v. sali'na, Bab. v. marit'ima $^{\top}$ (I.)

Parthen'ium, L. = Chrysanth'emum Parthen'ium, Pers.

Math'iola, K.Br. Crucr'Fers, Crucifers. Tetrad. Siliq. inca'na, R.Br. 3. s. 5, 6. Hoary Shrubby Stock. Gilliflower. simua'ta, ${ }^{8}$ R.Br. 7. b. 5-8. Great Sea Stock.

Me'conop'sis, Vig. Papa'vera'cee, Poppyworts. Polyan. Mon. camb rica, ${ }^{10}$ Vig. 14. p. 6. Yellow Welsh Poppy.

Me'dica'go, L. LEgo'mino's s, Leguminous Plants. Diad. Dec. arab'ica, All. See macula'ta, Sibth. dent'icula'ta, Willd. 18. a. or b. 5-8. Reticulated Medick. v. ap'icula'ta (Willd.)

v. lappa'cea (Desr.)

1134

1832

vii. 1064

xii. 1825

769

768

$768 \mathrm{~b}$

$768 \mathrm{c}$

(v.719)

(v.717)

(v.718)

74

73

58

i. 105

i. 104

48

49

i.63

41

335

$335 b$

$335 \mathrm{c}$

524

523

3 Majanthemum bifolium.6 C. inodorum. - Pyrethrum
1 Anchusa arrensis.

2 Herb Two-pence.-Creeping Loosestrife.

Imilacina bifolia. 4 Marsh Tuay-blade. 5 Chrysanthemum Chamomilla. inoriorum.
8 Cheiranthus incands. 9 C. sinuatns. 10 Papaver cumbricum. 
Me'dica'go, L.-eontinued.

L.C.

falca'ta, L. 5. p. 6,7. Yelbow Lucerne, or Sickle-podded Medick. lŭpuli'na, L. 100. a. or b. 5-8.'Black M. Nonsuch. Shamrock. ${ }^{1}$ macula'ta, ${ }^{2}$ Sibth. [arab'ica, All.] 40, a. or b.5-8. Spotted Medick. min'ima, Desr. 10. a. or b. 5-7. Little Bur Medick or Bur-weed. mu'rica'ta, Willd. a. 6, 7. Prickly Medick.

polymor.ph'a, L. = M. macula'ta, Sibth.

sati'va, L. p. 5-7. Common Luccrne. Purple Medick.

sylvest'ris, Fr. 4. p. 6, 7. Fries' Lucerne.

Mel'ampy'rum, Tourn. Scro'phularin'es, Figworts. Did. Ang. arrens'e, L. 7. a. 7,8 . Field or Purple Cow-wheat.

crista'tum, L. 10, a. 9, 10. Crested Cow-wheat.

pratens'e, L. 105. a. 6-9. Yellow Cow-wheat.

v. la'tifol'ium (Syme)

v. er'îceto'rum, D. Oliv.

r. monta'num, Johnst.

v. vulga're.

sylva'ticum, L. 21. a. 7, 8. Small-flowered Yellow Wood $C$.

Mel'ica, L. Grämis'ex, Grasses. Tri. Jig. cerul'ea, $\mathrm{L} .=$ Molin'ia cærul'ea, Mœnch.

nu'tans, L. 40. p. 5, 6. Nodding or Mountain Melic-grass. u'niflo'ra, Retz. 91. p. 5-7. Wood Melic-grass.

Melilo'tus, Tourn. Legu'Mro's.e, Leguminous Plants. Diad. Dec. alb'a, ${ }^{3}$ Desr. 40. b. 7, 8. White Melilot.

altiss'ima, Tbuili. [offic'îna'lis, Lam.] 72. b. or p. 6-8. Common Melilot.

arvens'is, Wallr. See officina'lis, Desr.

ina'ica, All. See parvitho'ra, Lam.

leucanth'a, Koch = M. alb'a, Desr.

offic'ina'lis, Desr. [arvens'is, Wallr.] b. 6.8. Field Melilot.

offic'ina'lis, Lam. See altiss'ima, Thuill.

parviflo'ra, Lam. [ind'ica, All.] a. 7. Small-flowered Melilot. vulga'ris, Wallr. = M. alb'a, I)esr.

Meliss'a, L. LAв ${ }^{\prime} \mathbf{A}^{\prime}$ т.

Ac'inos, Bentb. = Cal'aminth'a arrens'is, Lam.

Cal'aminth'a, L. = Cal'aminth'a offic'ina'lis, Mœnch.

Cli'nopod'ium, Benth. = Cal'aminth'a Cli'nopod'ium, Benth.

Nep'eta, L. = Cal'aminth'a Nep'eta, Clairv.

offe'ina'lis, L. p. 7, 8. Common Balm.

Melitt'is, L. Labia'tx, Labiates. Did. Gym.

grandiflo'ra, Sm. "a slight variety of M. Meliss'ophyll'um."

Meliss'ophyll'um, L. 9. p. 5, 6. Reddish Bastard Balm.

Menth'a, L. IAB' IA'TE, Labiates. Did. Gym.

acu'tifol'ia, Sm. p. 7, 8. (a form of M. sati'va) Sharp-leaved Mint. agrest'is, Sole $=\mathbf{M}$. arrens' is, v. agrest'is.

aló pecu'roï'des, Hull. 9. p. 8, 9. Brond-leaved Horse Mint. aqua'tica, Benth. $=$ M. hirsu'ta, Hnds.

arvens'is, L. 100. p. 8, 9. Corn Mint.

v. Nummula'ria (Schreb.)

v. agrest' is $^{5}$ (Sole)

v. præ'cox (Sole)

v. Alliónii (Bor.)

v. par'ieta'riæfol'ia (Beck.)

citra'ta, Ehrh. = M. hirsu'ta, v. citra'ta.

crisp'a, L. p. 8, 9. Curled Mint.

genti'lis, L. p. 8, 9. Bushy Red Mint.

v. Wirtgenia'na (F. Schultz.)

v. Paulia'na (F. Schultz.)
E.B.

iii. 336

iii.337

iii. 339

iii. 340

iii.334

331

iii. 335

1075 vi. 1001

1074 vi. 1000

1076 vi.1002-4

$1076 \mathrm{~b}$ vi. 1002

$1076 \mathrm{c}$

$1076 \mathrm{~d}$

1077

vi. 1004

vi. 1003

vi. 1005

1694

1695

xi. 1748

xi.1749

339

iii. 342

338

(iii.341)

iii. 343

340

(iii. 343 )

iii.341

341

iii. 344

1125

vii. 1053

vii. 1063

1133

vii. 1062

1103

vii. 1021

1114

$1114 \mathrm{~b}$

$1114 \mathrm{c}$

$1114 \mathrm{~d}$

$1114 \mathrm{e}$ vii. 1040

$1114 \mathrm{f}$

1113

$1113 \mathrm{~b}$

vii. 1029

vii. 1028

vii. 1037

$1113 c$ vii.1037
773

795

B.

233

235

237

238

234

771

770

772

1245

1246

241

(239)

240

(240)

239

781

783

1 Yellow Clover. 2 Medicago polymorpha. officinale. 5 Meutha agrestis. 
Menth'a, L.-continued.

gra'cilis, Sm. p. 8, 9. Slender or Narrow-leaved Red Mint.

v. carl'iaca, Baker. Cardiac Mint.

hirsu'ta, ${ }^{1}$ Huds. 104. p. 7, 8. Hairy Water Mint.

v. subgla'bra (Baker)

v. citra'ta ${ }^{2}$ (Ehrh.) Berganot Mint.

longifol'ia, ${ }^{3}$ Huds. 58. p. 8, 9. Common Horse Mint.

v. nem'oro'sa (Willd.)

v. molliss'ima (Borkh.)

P’uperi'ta, L. 64. p. 7, 8. Pepper-mint.

v. offic'ina'lis (Hull) Garden Pepper-mint.

v. vulga'ris (Sole) Wild Pepper-mint.

pratens'is, Sole. p. 8, 9. Meadow or Narrow-leaved Mint.

pubesc'ens, Willd. 4. p. 8,9. Blunt-spiked Mint.

v. palust'ris (Sole)

v. hirci'na (Hull)

Pule'gium, L. 51. p. 8, 9. Penny-royal.

v. decumb'ens.

v. erect'a (Syme)

rotund'ifol'ia, Huds. 50. p. 8, 9. Round-leaved Mint.

ru'bra, Sm. p. 8, 9. Tall Red Mint.

sati'va, L. 83. p. 7-9. Marsh Whorled Mint.

v. riva'lis, Lond. Cat.

v. paludo'sa (Sole)

v. subgla'bra, Baker.

sylrest'ris, $\mathrm{L}_{\mathrm{s}}=\mathbf{=}$. longifol'ia, Huds.

vir'idis, L. p. 8, 9. Garden or Spear Mint.

v. crisp $a$, Hook.

Me'nyanth'es, 'Tourn. Gextia'se, Gentianworts. Pent. Mon. nymplia'oï des, L. = Limnanth'emum pelta'tum, Gmel.

trifol'ia'ta, L. 106. p. 5-7. Buck-bean. Bog-bean. Marsh Trefoil.

Menzies'ia, Sm.

cæerul'ea, Sm. = Phyllod'ocē taxifol'ia, Salisb.

pul'iful'ia, Juss. = Jabœ'cia pol'ifol'ia, D. Don.

Merc'uria'lis, Tourn. Euphorbia'ce.e, Spurgeworts. Dioec. Enne. ambig'ua, L. = M. ann'ua, v. ambig'ua.

ann'ua, L. 42. a. 7-10. Annual Dog's Mercury.

v. ambig'ua ${ }^{4}$ (L. fil.)

perenn'is, L. 105. p. 4, 5. Perennial Dog's Mercury.

Mertens'ia, Roth. BonĀGin'ex, Borageworts. Pent. Mon. marit'ima, ${ }^{5}$ Gray. 29. p. 5, 6. Oyster-plant. Sea-Bugloss. ${ }^{6}$

Mesp'ilus, L.

Coto'neas'ter, L. = Coto'neas'ter vulga'ris, Liudl.

germa'nica, $\mathrm{L}$. $=$ Pyr'us germa'nica, $\mathrm{L}$.

Ux'yacanth'a, Willd. = Cratæ'gus Ox'yacanth'a, v. ox'yacan-

Me'um, Tourn. Unaellir'erse, Umbellifers. Pent. Dig. [th'oï'des. Athamant'icum, Jacq. 26. p. 6, 7. Bald-Money. Spignel. Meu. Fœ'niculum, Spreng. = Fœ'niculum offic'īna'le, All.

Mibo'ra, Adans. Gramin'e.e, Grasses. Tri. Dig. min'ima, Desv. $=$ M. vern'a, Adans.

rern'a, ${ }^{7}$ Adans. 3. a. 3, 4. Early Sand-grass.

Mi'crocal'a, Link. Gentínex, Gentianworts. Tetran. Mon. fi'liform' is, ${ }^{8}$ Link. 9. a. 7-9. Slender Cicendia. Gentianella.

Mil'ium, L. GRāmun'E, Grasses. Tri. Dig. effu'sum, L. 80. p. 5, 6. Wood Millet-grass. Spreading $M$. lendig'erum, L. = Gastrid'ium lendig'erum, Gaud.

Mim'ulus, L. Scro' Phularin'ed, Figworts. Did. Ang. lu'teus, L. p. 7-9. Yellow Monkey-flower.

I.C. E.B.

1111 vii. 1034

1111 b vii. 1035

1108 vii. 1030

$1108 \mathrm{~b}$

$1108 \mathrm{c}$ (vii.1029)

1104 (vii.1022)

$1104 \mathrm{~b}$

$1104 \mathrm{c}$

1106 vii. 1024,5

$1106 \mathrm{a}$ vii. 1024

1106 b vii. 1025

1112 vii. 1036

1107 vii. 1026

$1107 \mathrm{a}$

$1107 \mathrm{~b}$ vii. 1027

1115 vii. 1041,2

vii. 1041

$1115 \mathrm{~b}$ vii. 1042

1102 vii. 1020

1110 vii. 1033

1109 vii.1031

$1109 a$

$1109 \mathrm{~b}$

$1109 \mathrm{c}$

1105

$1105 \mathrm{~b}$

vii.1032

vii.1022

vii. 1023

777

779

$9 \% 9$

vi. 920

650

vi. 886

vi. 885

633

632

1262 viii. 1269

895

1262 b viii. 1270

1261 viii. 1268

993 vii.1099

894

692

iii. $4-8$

339

661

iv. 605

429

1653 (xi.1689)

(1172)

965 (vi.9l2)

1648 xi.1728

1152

1044 vi.967

743

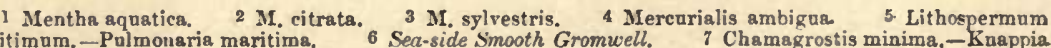
maritimum.-Pulmonaria maritima, 6 Sea-side Smooth Gromwell. 7 Chamagrostis minim
agrostidea.-Mibura minims. 8 Cicendia fliformis.-Exacum fliforme. 9 Marsh Centaury. 
Mœhring'ia, L.

trinerv'ia, Clairv. $=$ Ar $^{\prime}$ êna'ria trinerv'ia, $\mathrm{L}$.

Mœnch'ia, Fhrh.

erect'a, Sm. = Cerast'ium quāternell'um, Fenzl.

glauc'a, Pers. = Cerast'ium quāternell'um, Fenzl.

Molin'ia, Schrank. GräMin'ex, Grasses. Tri. Dig.

caerul'ea, ${ }^{1}$ Monch. 106. p. 7, 8. Purple or Blue Melic-grass.

v. depau'pera'ta ${ }^{2}$ (Lindl.)

depau'pera'ta, Lindl. =M. cærul'ea, v. depau'pera'ta.

Mone'ses, Salisb. ER' $\mathrm{E}^{\prime} \mathrm{CA}^{\prime} \mathrm{CE}$, Heathworts. Dec. Mon. grandiflo'ra, ${ }^{3}$ Gray. 9. b. 6, 7. Single-flowered Winter-green.

Monot'ropa, L.

Hýpopit'ýs, L. $=$ Hy̆p'opit'y̆s multiflo'ra, Scop.

Monotrop'e.e, Fir-Rapes.

Mont'ia, L. Portula'Ce.s, Purslanes. Tri. Trig.

fonta'na, L. 107. a. 5-8. Water Blinks. Water Chickweed.

v. min'or, Gmel. [re'pens, Pers.]

v. ri'vula'ris, Gmel. [erect'a, Pers.]

Mulge'dium, Cass.

alpi'num, Less. = Lactu'ca alpi'na, Benth.

Musca'ri, Tourn. Li'Lis'CEE, Lilyworts. Hex. Mon. rac'emo'sum, ${ }^{4}$ Mill. 3. p. 5. Starch Hyacinth. Grape $H$.

Myoso'tis, Dill. Borāin'ex, Borageworts. Pent. Mon. alpest'ris, ${ }^{5}$ Schmidt. 4. p. 7, 8. Alpine Forget-me-not. ${ }^{6}$ arvens'is, ${ }^{7}$ Willd. 111. a. or b. 6-8. Field Forget-me-not. r. umbro'sa, Bab.

Balbi'sia'na, Jord. 1.

crespito'sa, ${ }^{3}$ Schultz. 101. p ? or b. 5-8. Tufted. Water F. colli'na, Hoffm. 89. b. or p, 5-7. Dwarf F. Early Field $F$.

v. Mitte'nii, Lond. Cat.

intermed'ia, Link $=$ M. arvens' is, Willd.

lingula'ta, Lehm. $=$ M. cæspito'sa, Schultz.

palust'ris, Relh. 98. p. 5-7. Great Water $F$. Scorpion-grass. v. strĭgulo'sa, Mert. \& Koch.

re'pens, D. Don. 84. p. 6-8. Creeping Water Forget-me-not. rupic'ola, Sm. = MI. alpest'ris, Schmidt.

sylva'tica, Hoffm. 42, b. or p. 5, 6. Upright Wood Forget-me-not. versic'olor, Reichb. 103. b. or a.4-6. Yellow \& blue, or Changeable $F$. min'imus, L. 43. a. 4-6. Common or Little Mouse-tail.

Myri'ca, L. Mrrica'ces, Galeworts. Diœc. Tet.

Ga'le, L. 79. s. 5-7. Bog Myrtle. Dutch Myrtle. Swett Gale.

Mrrica'Ce.e, Galeworts.

Myr'iophyll'um, L. Halora'ges, Hippurids. Monœc. Pol. altern iflo'rum, DC. 69. p. 6-8. Alternate-flowered Water Milfoil. spica'tum, L. 65. p. 6-8. Spiked Water-Milfoil.

vert'icilla'tum, L. 49. p. 7, 8. Whorled Water-Milfoil. v. pectina'tum (DC.)

Myrrh'is, Tourn. Umbellif'ere, Umbellifers. Pent. Dig. ar'omat'ica, Spr. $=$ Chærophyll'um ar'omat'icum, L. aur'ea, Spr. = Chærophyll'um aur'eum, L.

Od'ora'ta, ${ }^{8}$ Scop. 60. p. 5, 6. Sweet Cicely.

te'mulent'a, Sm. = Chærophyll'um te'mulum, L.

Náiada'Ce, Juss. Naiads.

Na'ias, Willd. NA'IADA'CEe, Naiads. Monœc. Pent. flex'ilis, Rostk. 2. p. 8, 9. Flexible or Slender Naias.

1 Melica cæralea.

Syosotis rupicola.

2 Molinis depanperata. 3 Pyrola uniflora. odorata.
L.C.

$1692 \mathrm{~b}$

932

933

255

$255 a$

$255 b$

1408

1408

997

999

$999 \mathrm{~b}$

1002

994

1000

$1000 \mathrm{~b}$

995

995

996

vii. 1104

vii.1105

998

1001

vii.1107

vii. 1110

11

E.B.

B.

156

$x i .1747$

1244

(vi.900)

vi.901

645

ii. 259

173

จ. 809

ix. 1529

1042

vii.1106

vii. 1108

vii. 1103

vii. 1109

699

696

697

700

i.14

8

907

1270

580

579

578

$578 \mathrm{~b}$

iv. 515

iv. 514

iv. 513

392

393

641

iv. 626

439

ix. 1399

\begin{tabular}{l|l}
1516 & ix. 1432
\end{tabular}

950

4 Hyacinthus racemosus. 8 M. lingulata, 9 Scandix 
Na'ias, Willd._-continued. [grämin'ea, Del.]

v. Delil'ei, Magnus.

mari'na, I. 1. p. 7.

Narciss'us, L. Am'arylud'Ee, Amaryllids. Hex. Mon.

biflo'rus, Curtis. p. 4, 5. Two-flowered Narcissus. Pale N.1

incom'para'bilis, Mill. p. 4. Short-crowned Daffodil.

lob'ula'ris, Schult. p. 3-5. Tenby Six-lobcd Daffodil.

$m a^{\prime}$ jor, L. p. 3. Large Narcissus.

min'or, I. p. 3. Dwarf Daffodil.

poe'ticus, L. p. 5. Poet's Narcissus. Pheasant's-eye N.

Psend'o-narciss'us, L. 76. p. 3, 4. Daffodil. Lent Lily.

v. lob'ula'ris, Haw.

Nard'us, L. Grämin'ex, Grasses. Tri. Mon.

strict'a, L. 107. p. 6, 7. Mat-grass. Mat-weed. Nard.

Narthe'cium, Huds. Li'LIs'CE., Lily-worts. Hex. Mon.

ossif' ragum, Huds. 95. p. 7, 8. Lancashire Bog Asphodel.

Nasturt'ium, R.Br. Crucif'ERe, Crucifers. Tetrad. Siliq. amphib'ium, ${ }^{3}$ R.Br. 44.p. 6-9. Great, or Amphibious Yellow-cress. ${ }^{4}$ offic'ina'le, ${ }^{5}$ R.Br. 109. p. 5-10. Commmon Water-cress.

v. si'ifol'ium (Reichb.) Koch.

v. mi'crophyll'um (Buenn.) Reiclib.

palu it're, ${ }^{6}$ DC. 79 . p. 6-10. Water-cress. Annual Yellow-cress, or Rocket. ${ }^{7}$

sylvest're, ${ }^{6}$ R.Br. 59. p. 6-8. Crecping Yellow-cress. ${ }^{8}$ terrest're, R.Br. $=N$. palust're, DC.

Neotin'ea, Reichb. fil. intact'a, Reichb. fil. = Hăbena'ria intact'a, Benth.

Neott'ia, L. Orchin'E.E, Orchids. Gyn. Mon. æstiva'lis, DC. = Spiranth'es æstiva'lis, Rich. cern'ua, Willd. $=$ Spiranth'es Romanz'ovia'11a, Cham. gemmip'ara, Sw. = Spiranth'es Romanz'ovia'na, Cham. Ni'dus-av'is, ${ }^{10}$ Rich. 81. p. 6, 7. Bird's-nest Orchis. spira'lis, Sw. = Spiranth'es autumna'lis, Rich.

Nep'eta, L. LAB'IA'Ts, Labiates. Did. Gym.

Cata'ria, L. 56. p. 7-9. Cat-mint. Cat-nep. Nep.

Glecho'ma, ${ }^{11}$ Bentl. 102. p. 3-6. Ground Ivy. Ale-hoof. Gill. v. parviflo'ra, Benth.

Něphro'dium, Rich.

$æ^{\prime}$ mulum, Baker $=$ Lastræ'a $æ^{\prime}$ mula, Brackenbridge.

crista'tum, Rich. = Lastra'a crista'ta, Presl.

di'lata'tum, Desv. = Lastræe'a di'lata'ta, Presl.

Fil'ix-mas, Rich. = Lastræ'a Fil'ix-mas, Presl.

Or'eopt'eris, Desv. = Lastræe'a Or'eopt'eris, Presl.

remo'tum, Hook. fil. = Lastræ'a remo'ta, Moore.

rig'idum, Desv. = Lastræ'a rig'ida, Presl.

spi'nulo'sum, Desv. = Lastre'a spi'nulo'sa, Presl.

Thēlypt'eris, Desv. = Lastræ'a Thēlypt'eris, Presl.

üli'gino'sum, Desv. = Lastræe'a ūli'gino'sa, Newm.

Nitell'a, Agardh. Chiari'Cex, Charads. Crypt. Char. capita'ta, Agardh. 1. a.

flex'ilis, ${ }^{12}$ Agardh. 24. p. 7. Smooth Water Horsetail. ${ }^{13}$

v. crass'a, $\mathrm{A}$. Br.

v. nidif'ica, Wallm. \begin{tabular}{l|l|l} 
glom'era'ta, Clievallier = Tol'ypell'a glom'era'ta, Leonh. & & xii. 1905,6 \\
grac'ilis, ${ }^{14}$ Agardh. 2. 2.9. Slender Nitella or Water Horsetail. & 1853 & xii.1903
\end{tabular}

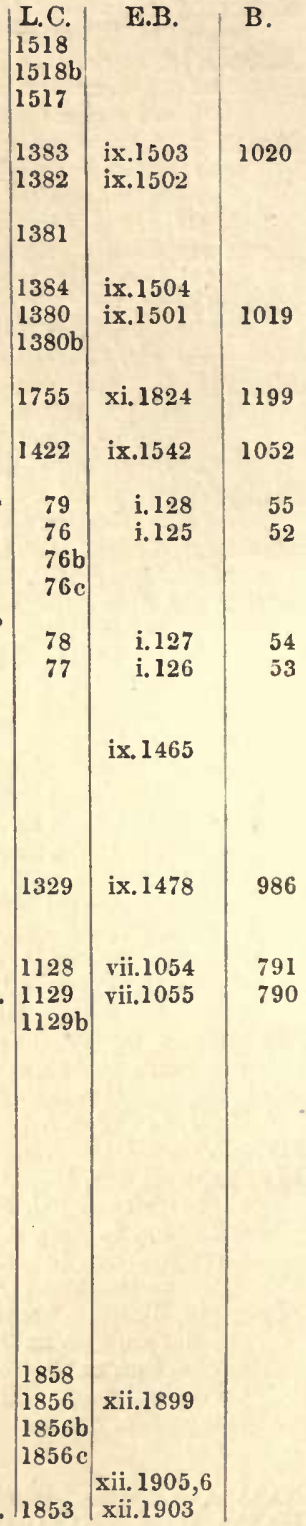

1 Primrose Peerless.

2 Daffy-down-dilly.

3 Armoracia amphibia.-Sisymbrium amphibium. 4 Great Water Rocket, or Radish. ${ }_{5}$ S. Nasturtium. 6 S. terrestre. - Nasturtium terrestre. 7 Marsh Nasturtium.-Small Jagged Water Radish. 8 Sisymbrium sylvestre. 9 Water Rocket.-Wild Nasturtium. 10 Listera Nidus-avis. -Ophrys Nidus-aris 11 Glechoma hederacea. 12 Chara flexilis. 13 Flaccid Nitella.

14 Chara gracilis. 
Nitell'a, Agardh._-continued.

intrica'ta, Agardh $=$ Tol'ypell'a intrica'ta, Leonh.

v. prolif'era = Tol'ypell'a prolif'era, Leonh.

nidif'ica, Agardh = Tol'ypell'a nidif'ica, Leonh.

mu'crona'ta, ${ }^{1}$ Kuetz. 2. a. 7. Mucronate Nitella.

opa'ca, ${ }^{2}$ Agardh. 61. a. or p. 5, 6. Twin-fruited Nitella.

v. atten'ua'ta, H. \& J. Groves.

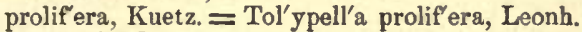

sy̆ncarp'a, Cher.

v. opa'ca $=$ N. opa'ca, Agardh.

ten'uiss'ima, ${ }^{3}$ Kuetz. 2. a. 8. Dwarf Nitella.

translu'cens, ${ }^{4}$ Agardh. 20. p. 8. Translucent Nitella. ${ }^{5}$

Nu'phar, Sm. NympheA'CEe, Water-Lilies. Polyan. Mon.

lu'teum, Sm. 89. p. 6-8. Yellow Water-Lily. Water-ean. ${ }^{\circ}$

v. intermed'ium, Auct. Angl.

v. ma'jor.

v. min'or.

min'ima, Sm. $=$ N. pu'milum, Sm.

pu'milum, ${ }^{7} \mathrm{Sm}$. 12. p. 6-8. Least Yellow Water-Lily.

Nymphæ'a, L. NympiraA'ce.s, Water-Lilies. Polyan. Mon. alb'a, L. 83. p. 6-8. White Water-Lily.

v. min'or, DC.

NYMPH.EA'CE.E, DC. Water-Lilies.

Obio'ne, Grert.

pedunc'ula'ta, Moq. = Atrip'lex pedunc'ula'ta, $L$.

port'ula'coi'des, Moq. = Atrip'lex port'ula'coï'des, L.

Od'onti'tes, Duby.

ru'bra, Pers. = Barts'ia Od'onti'tes, Huds.

Enanth'e, L. Umbellif'er. Umbellifers. Pent. Dig. croca'ta, L. 89. p. 7. Hemlock Water-Dropwort.

fistulo'sa, I. 65. p. 7-9. Common Water-Dropwort.

fluvia'tilis, Coleman. 25. b ? or p. 7-9, River Water-Dropwort.

Lachenal'ii, Gmel. 70. p. 7-9. Parsley Water-Dropwort. peuc'edan'ifol'ia," " Poll." 21.p.6. Sulphur-wort Water-Dropwort. Phelland'rium, ${ }^{8}$ Lam. 56. b. or p. 7-9. Fine-leaved $W . D$. pim'pinell'oï'des, L. 16. p. 6-8. Callous-fruited $W . D$. sil'aifol'ia, Bieb? = CE. peuc'edan'ifol'ia, " Poll."

Enothe'ra, L. ON'AGra'rie.e, Onagrads. Oct. Mon. bienn'is, L. b. 7-9. Common Evening-Primrose. od'ora'ta, Jacq. p. 7-9. Sweet-scented Evening-Primrose.

OL'EA'CEs, R.Br. Olive-worts.

ON'AGRAR'IEe, Juss. Onagrads.

L.C.

1854

1857

$1857 \mathrm{~b}$

1858

1852

1855

651

$65 t$

653

656

606 vic'iæfol'ia, Scop. See sati'va, Lam.

Ono'nis, L. Legu'mó's.e Leguminous Plants. Diad. Dec. antiquo'rum, L. $=0$, spino'sa, $\mathrm{L}$.

arvens'is, Fries. $=0$. re'pens, $\mathrm{L}$.

campest'ris, Kinch. $=0$. spino'sa, $\mathrm{L}$.

procurr'ens, Wallr. $=0$. re'pens, $\mathrm{L}$.

rec'lina'ta, L. 2. a. 6, 7. Small Spreading Rest-harrow.

re'peus, ${ }^{12}$ L. 99. p. 6-9. Procumbent $R$. Wild Liquorice. ${ }^{13}$

v. inerm' is, Lange.

v. horr'ida, Lange.

spino'sa, ${ }^{1+}$ L. 69. p. 6-8. Upright Spiny Rest-harrow.

v. mi'tis (Gmel.)
E.B.

xii. 1907

xii. 1908

xii. 1902

(xii.1900)

50

$50 \mathrm{~b}$

i. 54,5

i. 54

i.55

51

i. 56

52

$52 \mathrm{~b}$

i.53

50

i.53

655

iv. 597

422

iv. 593

iv.599

iv. 596

(iv.595)

iv. 598

iv. 594

iv. 595

605

iv. 508

iv. 509

961

iv. 494

380

iii.381

275

B.

423

421

349

iii. 331

iii. 330

329

iii.332

327 (iii.331)

232

(23I)

- Chara mucronata. 2 C. syncarpa,-Nitella synesrpa, v, opaca, 
On'opord'on, L. Compos'ıxs, Composites. Syn. Aqq. Acanth'ium, L. 60. b. 7-9. Scotch Thistle. Cotton T. ln'sita'nicum, L. C. p. 1, 2. Dwarf or Lesser Adder's-tongue. vulga'tum, L. 87. p. 5-7. Common Adder's-Tongue. v. polyphyll'um, A.Br. 3 .

Ophiu'rus, Beanv.

incurva'tus, Lindl. = Leptu'rus fi'liform'is, Trin.

Oph'rȳs, L. Orchid'ex, Orchids. Gyn. Mon.

an'thrōpoph'ora, I. $=$ Ac'eras an'thrōpoph'ora, R.Br.

apif'era, Huds. 48, p. 6, 7. Bee Orchis.

v. Troll'ii, Heg.

arachni'tes, ${ }^{2}$ Reichb. 3. p. 4-6. Late Spider Orchis.

arānif'era, Huds. 12. p. 4,5. L arly Spider Orchis. Bee Ophrys.

v. fucif'era, ${ }^{3} \mathrm{Sm}$. Drone Orchis.

Corall'orbi'za, L. $=$ Corall'orhi'za inna'ta, R.Br.

corda'ta, L. = Liste'ra corda'ta, R.Br.

fucif'era, $\mathrm{Sm}_{.}=$O. arānif' era, $\mathrm{v}$. fucif' era.

fu'ciflo'ra, Reichb. f. $=0$. arachni'tes, Reichb.

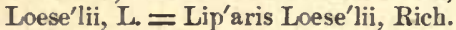

Monorch'is, $\overline{\mathrm{L}}$. $=$ Hermi'nium Monor'chis, R.Br.

muscif'era, Huds. 42. p. 5, 6. Fly Orchis, or Ophrys.

my̌o'dēs, Jacq. = O. muscif'era. Huds.

Ni'dus-av'is, Hook. $=$ Neott'ia Ni'dus-av'is, Rich.

ova'ta, L. = Liste'ra ova'ta, R. Br.

spira'lis, L. = Spiranth'es autumna'lis, Rich.

Oporínia, Don.

antumna'lis, Don. = Leont'odōn autumna'lis, $\mathrm{L}$.

(ORCHID'E,E, Orchids.

Orch'is, L. Orchid'ex, Orchids. Gyn. Mon.

alb'ida, Scop. = Hăbena'ria alb'ida, R.Br.

bifol'ia, L. = Hăbena'ria bifol'ia. R.Br.

cōno'psea, L. = Hăbena'ria cōno' psea, Bentb.

fusc'a, Jacq. $=0$. purpur'ea, Huds.

hirci'na, ${ }^{5}$ Scop. 4. p. 4-8. Lizard Orchis.

incarna'ta, L. 57. p. 6, 7. Common Marsh Orchis. v. angust'ifol'ia, Bab.

intact'a, Link = Hăbena'ria intact'a, Benth.

la'tifol'ia, L. 32. (agg. 105) p.5-7. Broad-leaved Marsh Orchis. laxiflo'ra, Lam. C. p. 5, 6. Lax-flowered Marsh Orchis.

ma'cra, Lindl. = O. Si'mia, Lam.

macula'ta, L. 108. p. 5-7. Spotted Palmate Orchis.

masc'ula, L. 100. p. 4-6. Early Purple Orchis.

milita'ris, L. 5. p. 5, 6. Military Orchis.

Mo'rio, L. 63. p. 5, 6. Green-winged Meadow Orchis.

palma'ta, Syme.

purpur'ea, ${ }^{6}$ Huds. 3. p.5. Great Dark-, or Brown-winged 0. py'ramida'lis, ${ }^{7}$ L. 63. p. 6-8. Pyramidal Orchis.

Si'mia, Lam. 3. p. 5. Monkey Orchis.

teph'rosanth'os, Vill. = O. Si'mia, Lam.

ustula'ta, L. 42. p. 5, 6. Dwarf Dark-winged Orchis.

Orig'anum, L. LAB'IA'Tx, Labiates. Did. Gym.

vulga're, L, 89. p. 7-9. Wild Marjoram.

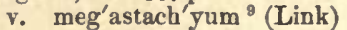

v. prisma'ticum, Gaud. = v. meg'astach'yum.

Ornithog'alum, L. LI'LIA'CE E, Lilyworts. Hex. Mon. lu'teum, L. = Ga'gea fascicula'ris, Salisb.

nu'tans, L. p. 4, 5. Drooping Star of Bethlehem. \begin{tabular}{|l|r|r} 
L.C. & E. B. & B. \\
811 & v. 680 & 567 \\
1808 & xii.1836 & \\
1807 & xii.183j & 1269 \\
$1807 \mathrm{~b}$ & & \\
& & \\
& & \\
& & \\
1358 & ix.1467 & 1008 \\
$1358 \mathrm{~b}$ & & \\
1359 & ix.1468 & \\
1360 & $\mathrm{ix.} .1469$ & 1009
\end{tabular}

\begin{tabular}{l|l|l}
1360 & ix.1469 & 1009 \\
$1360 b$ & ix. 1470 &
\end{tabular}

1361

ix. 1471

1010

1326

ix. 1447

1002

1345 ix.1448 1000

1354 ix. 1457

$1354 \mathrm{~b}$

1355

1353

ix. 1458

ix.1456

999

997

1356

1352

1349

ix. 1459

ix. 1455

ix. 1452

998

996

993

1351

ix. 1454

ix. 1457,8

1348 ix.1451

1346 ix.1449

1350 ix.1453

1347 ix. 1450

994

1117 vii. 1045

786

$1117 \mathrm{~b}$

(vii.1046)

vii. 1046

1412 
Ornithog'alum, L.-continued.

pyrena'icum, L. 8. p. 6, 7. Tall or Spiked Star of Bethlehem.

umbella'tum, L. p. 5, 6. Common Star of Bethlehem.

Orni'thopūs, L. LEGU'Mrvo's.e, Leguminous Plants. Diad. Dec. ëbract'ea'tus, ${ }^{1}$ Brot. 1. a. 6-8. Sand Bird's-foot or Joint Vetch. per'pusill'us, L. 83. a. 5-7. Least Bird's-foot.

OR'OBancha'ces, Vent. Broom-rapes.

Orobanch'e, L. Oz'obancha'cee, Broom-rapes. Did. Ang. amethyst'ea, Thuill. p. 7. Bluish Broom-rape.

ar'ēna ria, Borkh. C. ? p. 7, 8. Sand Broom-rape. cæerul'ea, Vill. [purpur'ea, Jacq.] 8.p. 6-10. Purple Broom-rape. car'yophylla'cea, Sm. 2. p. 6. Clove-scented Broom-rape.

ela'tior, Sutton. 25. p.6-8. Tall Broom-rape.

Hed'eræ, Duby. 20. p. 6-8. Ivy Broom-rape. ma'jor, ${ }^{2}$ L. 58. p. 6-8. Greater Broom-rape. min'or, Sm. 29. p. 6-10. Lesser Broom-rape. v. flaresc'ens, Reuter. C.

Pi'cridis, F. Schultz. 5, a. 6, 7. Picris Broom-rape. purpur'ea, Jacq. See cærul'ea, Vill.

ramo'sa, L, a. 8, 9. Branched Broom-rape.

ra'pum, Thuill. $=0$. ma'jor, $\mathbf{L}$.

ru'bra, Stn. 12. p. 6-8. Red Broom-rape.

Or'obus, L.

nig'er, L. = Lath'yrus nig'er, Wimm.

sylva'ticus, L. = Vic'ia Or'obus, DC.

tubero'sus, L. = Lath'yrus ma'crorrhi'zus, Wimm.

Osmund'a, L. FiL'ices, Ferns. Crypt. Fil.

Luna'ria, L. = Botrych'ium Luna'ria, Swartz.

rega'lis, L. 85. p. 6-8. Flowering Fern. Royal Fern.

Ox'alis, L. Geran'in'cex, Cranesbills. Dec. Pent.

Ace'tosell'a, L. 109. p. 4-8. Wond Sorrel. Shamrock. corni'cula'ta, L. a. 6-9. Procumbent Yellow Wood-Sorrel. strict'a, L. a. 6-9. Upright Yellow Wood-sorrel.

Oxycocc'us, Tourn.

Oxplust'ris, Pers. = Scholle'ra Oxycocc'us, Roth.

Oxyr'ia, Hill. Pol'ygona'ce., Buckwheats. Hex. Dig. digyn'a, ${ }^{3}$ Hill. 25. p. 7, 8. Kidney-leaved Mountain-Sorrel. re'niform'is, Hook. = O. digyn'a, Hill.

Oxytrop'is, DC. Leg U'Mixo's.e, Leguminous Plants. Diad. Dec. campest'ris, ${ }^{4}$ DC. 1. p. 6, 7. Pale-yellow Oxtropis. ${ }^{5}$ Halle'ri, Bunge $=0$. u'ralens'is, DC.

u'ralens' is, ${ }^{8}$ DC. 9. 9. 6, 7. Blue, or Purple-flowered Oxytropis. ${ }^{7}$

Pæónia, L. Randnc'ola'cex, Crowfoots. Polyan. Pent. coralli'na, ${ }^{9}$ Retz. p. 5, 6. Entire-leaved Male or Coral Paony. offic' 'ina'lis, L. $=$ P. coralli'na, Retz.

Pa'nicum, L. Gramin'es, Grasses. Tri. Dig.

Crūs'gall'i, ${ }^{9}$ L. a. 7. Loose Panic-grass. Cockspur Panicum.

Dact'ylon, L. = Fibich'is umbella'ta, Koel.

gla'brum, ${ }^{10}$ Gaud. 5. a. 7, 8. Glabrous Finger-grass.

glauc'um, L. = Seta'ria glanc'a, Beauv.

sanguinu'le, ${ }^{11}$ L. a. 7, 8. Cock's-foot $F$. Đairy F. Red $F$. verc'icilla'tum, $\mathrm{L} .=$ Seta'ria vert'icilla'ta, Beaur.

vir'ide, L. = Seta'ria vir'idis, Beauv.

Papa'ver, L. Papa'vera'cex, Poppyworts. Polyan. Mon. Argemo'ne, L. 84.a. 5-7. Long Prickly-headed or Pale Poppy. camb'ricum, L. = Me'conops' is camb'rica, Vig.
L.C.

1414

1413

E.B.

ix. 1525

ix. 1524

377

376

1080

1090

1080

1081

1085

1086

1088

1083

1089

$1089 \mathrm{~b}$

1087

1082

1084

vi.1007
vi.1010
vi.1111

vi.1007
vi.1010
vi.1111

vi.1007
vi.1010
vi.1111

iii.379

iii. 378

vi. 1006

vi. 1017

vi. 1008

vi. 1009

vi. 1012

vi.1013

vi. 1015

(vi.1010)

vi.1016

vi. 1014

721

717

1806

xii. 1838

1271

308

ii. 310

215

309

ii.311

216

310

ii. 312

1220

(viii.1225)

(825)

viii.12.25

825

375

iii.374

iii.373

374

(iii.373)

270

271

47

i 50

1628

(xi.1692)

1158

1627

(xi.1691)

1154

1156

1626

1153

1155

1157

56

i. 61

B.

036

72 73 20

18

715

19

7 
Papa'ver, L. $\rightarrow$ continued.

dub'ium, L. 100, a. 5-7. Long Smooth-headed Poppy. v. Lecoq' $\mathrm{ii}^{1}$ (Lamot.) 22.

hortens'e, Hussenot. A sub-sp. of $\mathrm{P}$. somnif'erum, $\mathrm{L}$.

hyb'ridum, L. 39. a. 5-7. Round Prickly-headed Poppy.

Lamott'ei, Boreau. $A s u b-s p$. of $\mathrm{P}$. dub'ium, L.

Lecoq'ii, Lam. = P. dub'ium, v. Lecoq'ii.

nu'dicau'le, L. p. 7. Naked-stalked Yellow Poppy.

offic'ina'le, Gmel. A sub.sp. of $\mathrm{H}$. somnif'erum, $\mathrm{L}$.

Rhœ'as, L. 102. a. 6-8. Field or Red Poppy. Corn Rose.

v. strigo'sum (Boenn.)

somnif'erum, L. a. 7, 8. Garden Poppy. White P. Opium P. ${ }^{2}$

v. hisp'idum, H. C. Wats.

v. gla'brum, H. C. Wats.

Papa'veráces, Juss. Poppyworts.

Par'ieta'ria, Tourn. URTİCA'CEx, Nettleworts. Tetran. Mon. diffu'sa, Koch. = P. offic'ina'lis, L.

erect'a, Bab. $=\mathrm{P}$. offic'ina'lis, v. fall'ax.

offic'ina'lis, ${ }^{3}$ L. 91. p. 6-10. Pellitory-of-the-Wall.

v. fall'ax, ${ }^{4}$ Gr. et Godr.

Par'is, L. L'LIA'ces, Lily-worts. Oct. Tet.

quadrifol'ia, L. 72, p. 5, 6. Herb Paris. True Love. One-berry.

Parnass'ia, 'Tourn. SAxırrag'ex, Saxifrages. Pent. Tet. palust'ris, L. 81. p. 8, 9. Grass of Parnassus. ${ }^{5}$

Pastina'ca, L.

sati'va, L. = Peuc'edan'um sati'vum, Benth.

Pedi'cula'ris, Tourn. Scro'phularin'e.e, Figworts. Did. Ang. palust'ris, L. 107. b. or p. 5-9. Upright Marsh Loustwort. ${ }^{6}$ sylva'tica, L. 112. p. or b. 4-7. Procumbent Field Lousewort.

Pep'lis, I. LүтнRA'rise, Loosetrifes. Hex. Mon. Port'nla, L. 95. a. 7, 8. Water Purslane.

Peristy'lus, Blume.

alb'idus, Lindl. = Hăbena'ria aJb'ida, R.Br.

vir'idis, Lindl. = Hăbena'ria vir'idis, R.Br.

Pĕtasi'tes, Tourn. Compos'iтæ, Composites. Syn. Sup. alb'us, Grertn. p. 3. White Coltsfoot. fra'grans, ${ }^{\top}$ Presl. p. 2. Sweet-seented C. or Winter Heliotrope. offic'ina'lis, Mœnch. Sce vulgaris, Desf.

vulga'ris, ${ }^{8}$ Desf. [offic'ina'lis, Mœnch.] 102. p. 4, 5. Butter-bur.

Pet'roseli'num, Hoffm.

sati'vum, Hoffm. = Car'um Pet'roseli'num, Benth. et Hook. f. seg'etum, Koch = Car'um seg'etum, Benth. et Hook. f.

Peuc'edan'um, L. Umbeli'ifere, Umbellifers. Pent. Dig. offic'ina'le, L. 2. p. 7-9. Sea Hog's Fennel or Sulphur-wort. Ostru'thium, ${ }^{9}$ Koch. p. 7, 8. Broad-leaved $H$. Master-wort. ${ }^{10}$ palust're, ${ }^{11}$ Mœnch. 12. p. 9, 10. Marsh $H$. Milk Parsley. sati'vum, ${ }^{12}$ Benth. 57 . b. or a. 7,8 . Wild Parsnip.

Sil'ăus, L. = Sil'ăus pratens'is, Bess.

Phal'aris, L. Grāmus'E, Grasses. Tri. Dig. ar'èna'ria, Huds. $=$ Phle'um ar'ēna'rium, L.

arund'inu'cea, ${ }^{13}$ L. 109. p. 6, 7. Ribbon-grass. ${ }^{14}$

cana'riens'is, L. a. 7. Cultivated Canary-grass.

päradox'a, L. a. 7.

phleoi'des, L. = Phle'um phal'aroï'des, Koel.

Phēgopt'eris, Fée. Fin'rces, Ferms. Crypt. Fil.

calca'ren, Fée. See Robert'ia'na, A.Br.
Dry̆n t'eris, ${ }^{15}$ Fée. 69.p.7, 8. Oak Fern. Slender 3-branched Poly-

\begin{tabular}{|r|} 
L.C. \\
55 \\
55 \\
\\
57 \\
\\
\\
\\
54 \\
54 \\
53 \\
53 \\
53
\end{tabular}

53

i.57

viii. 1278

1269 (viii.1278)

$1269 \mathrm{~b}$

1424

ix.1509

1024

555

iv. 565

iv. 612

388

435

1072

1073

vi.996

vi.997

589

iv. 493

768

769

355

778

776

v.782

v.781

777

v. 783,4

iv. 576

iv. 577

666

iv. 609

668 iv.611

667 iv. 610

669 (iv.612)

432

434

433

(435)

1638

1636

(xi. 1697)

(1162)

1637

xi. 1698

1161

(1275)

1 Papaver Lecoqii. 2 Sleep-bearing Poppy. 3 Parietaria diffusa.

4 P. erecta.

5 Marsh

Parnassia. $\quad 6$ Red Rattle. 7 Tussilago fragrans, $\quad 8$ T. Petasites.-T, hybrida. 9 Imperatoria Ostruthium. 10 Herb Gerard. 11 Selinum palustre. 12 Pastinaca sativa, 13 Digraphis arandinacea, 14 Reed Canary-grass. 15 Polypodium Dryopteris. 
Phe̋gopt'eris, Fée.-continued. polypod'ioìdes, ${ }^{1}$ Fée. 73. p. 6-8. Beech Fern. Pale Mountain P. ${ }^{2} 1804$ Robert'ia'na, ${ }^{3}$ A.Br. [calca'rea, Fée.] 24. p. 7, 8. Limestone P."

Phelland'rium, $\mathrm{L}$. aqua'ticum, L. = Enanth'e Phelland'rium, Lam.

Phle'um, L. Gräun' Ex, Grasses. Tri, Dig.

alpi'num, L. 4. p. 7. Alpine Cat's-tail, or Timothy-grass. $a^{\prime} \overline{e n a}^{\prime}$ rium, ${ }^{5}$ L. 45. a. 5, 6. Sea-side Cat's-tail-grass. asp'erum, Jacq. a. 7. Rough Branched Cat's-tail-grass.

Bohme'ri, Schrad. = P. phal'aroi'des, Koel.

Miche'lii, All. p. 7, 8. Michelian Cat's-tail-grass.

pratens'e, L. 104.p. 6-8. Conmon Timothy-grass. Cat's-tail-grass.

v. nodo'sum (L) .

v. stol'onif'erum, Bab.

phal'aroi'des, ${ }^{8}$ Koel. 6. p. 7. Purple-stalked Timothy-grass.

Phragmi'tes, Trin. Gramin'ex, Grasses. Tri. Dig. commu'nis, ${ }^{7}$ Trin. 104. p. 7,8. Ditch Reed.

v. ni'gricans, Gren, et Godr.

v. re'pens, Mey.

Phyllod'ocē, Salisb. ER'îca'ces, Heathworts. Oct. Mon. carul'ea, Bab. = P. taxifol'ia, Salisb.

taxifol'ia, ${ }^{8}$ Salisb. 1. s. 6. Yew-leaved Menziesia. Scottish M.

Phys'osperm'um, Cuss. Umbellif'er.e, Umbellifers. Pent. Dig. commuta'tum, ${ }^{9}$ Spreng. 3. p. 7, 8. Cornish Bladder-sced. ${ }^{10}$ cornŭbiens'e, DC. $=$ P. commuta'tum, Spreng.

Phyteu'ma, L. Campa'ndla'cee, Bellworts. Pent. Mon. orb'icula're, L. 9. p. 7,8. Round-headed Rampion. spica'tum, L. 1. p. 5, 6. Spiked Rampion.

Pi'cris, L. Compos'1т ech'ioï'des, ${ }^{11}$ L. 65. a. or b. 6-10. Bristly Ox-tongue. Helminth. hiera'cioï'des, L. 60. b. 7-9. Hawk-weed. Fellow Succory. ${ }^{12}$ v. arva'lis (Jord.)

Pilulária, L. MAR'silea'ces, Pepperworts. Crypt. Fil. glŏbulit'era, L. 56. p. 6-8. Creeping Pillwort, or Pepper-grass.

Pimpinell'a, L. UMBeLlif'er., Umbellifers. Pent. Dig. dioi'ca, Sm. = Tri'nia vulga'ris, DC.

$\operatorname{mag}^{\prime}$ na, L. $=$ P. ma'jor, Huds.

major, ${ }^{13}$ Huds. 50. p. 7, 8. Greater Burnet Saxifrage. v. dissect'a, Wallr.

Saxif'raga, L. 99. p. 7. Common Bumet Saxifrage.

v. ni'gra (Willd.)

v. dissect'a, Spreng.

Pinguic'ula, Tourn. Lent'ibdra'ries, Butterworts. Dian. Mon. alpi'na, L. 3. p. 5, 6. Alpine Butterwort.

grandifio'ra, Lam. I. p. 5, 6. Lurge-flowered, or Irish $B$.

lu'sita'nica, L. 28. p. 6-10. Pale Butterwort.

vulga'ris, L. 91. p. 5-7. Common Butterwort.

Pi'nus, Tourn. Cons'ere, Conifers. Monce. Monad. Pinast'er, Ait. t. 4. Chester Pine.

Pi'sum, $\mathbf{L}$. sylvest'ris, L. 12, t. 5, 6. Scotch Fir. Scotch Pine.

marit'imum, L. = Lath'yrus marit'imus, Bigel.

Plantägin'en, Juss. Ribworts.

Planta'go, L. Plantāgin'ese, Ribworts. Tetran. Mon. ar'éna'ria, Waldst. et Kit.

argent'ea, Chaix.
L.C.

E.B.

xii. 1847

xii.1846

1649

1652

xi. 1705

1164

xi.1709 1167

xi.1708 1165

1650

xi. 1706

1163

$1650 \mathrm{~b}$
$1650 \mathrm{c}$

1651

1687

$1687 \mathrm{~b}$

$1687 \mathrm{c}$

926

(vi.886)

616

(iv.630)

iv. 630

(451)

451

899

900

vi. 864

612

vi. 865

613

827

(v.797)

v.796

(577)

826

$826 \mathrm{~b}$

1832

xii.1825

1268

iv. 586

415

639 (iv.586)

iv. 585

414

$638 \mathrm{~b}$

$638 \mathrm{c}$

1099

vii. 1123

1098

vii. 1122

1100 vii.1124

1097 vii.1121

1322

1321

viii.1381

viii. 1380

933

1 Polypodinm Phegopteris

2 Smith's Fern.

3 Polypodinm calesreum,-P. Robertianum.

- Rigid 3-branched Polypody. 5 Achnodon arensrins, - Phalaris arensria. 6 Phalaris phleoides, - Phleum Bahmeri. 7 Arundo Phragmites. 8 Menziesia carulea,-Phyllodoce carules. 9 Lignsticum cornubiense,-Physospermum cornabiense. 10 Cornish Lovage. 11 Ilelminthia echioides. 12 Ox-tongue. 13 Pimpinella magna. 
Planta'go, L. - continued.

Coro'nopius, L. 96. b. 6-8. Buck's-horn Plantain. ${ }^{1}$

lanceola'ta, L. 112. b. or p. 5-10. Rib-grass. Ribwort Plantain.

v. vulgáris.

v. Timba'li, ${ }^{2}$ Reichb. f.

ma'jor, L. 112. p. 5-9. Greater Plantain. Way-bread.

v. intermed'ia (Gilib.)

Inarit'ima, L. 78. p. 6-9. Sea-side Plantain.

v. min'or, Hook. \& Arn.

v. hirsu'ta.

v. la'tifol'ia.

med'ia, L. 81. p. 6-10. Goary Plantain. Lamb's-tongue.

'Timba'li, Jord. = P. lanceola'ta, v. Timba'li.

Plat'anthe'ra, Rich.

bifol'ia, Reichb. = Hăbena'ria bifol'ia, R.Br.

chlōranth'a Cust. = Hăbena'ria chlo'roleuc'a, Ridley.

Plumbiatin'te, Juss. Leadworts.

Po'a, L. Grāmin'ex, Grasses. Tri. Dig.

alpi'na, L. 15. p. 6-8. Alpine Meadow-grass.

ann'ua, L. 110. a. or b. 4-9. Annual Meadow-grass.

aqua'tica, L. = Glycer'ia aqua'tica, Sm.

Balfour'ii, Bab. $100^{3}$ p. 7. Balfour's Meadow-grass.

Borre'ri, Hook. \& Arn. = Glycer'ia Borre'ri, Bab.

bulbo'sa, L. 7. p. 4, 5. Bulbones Meadow-grass.

cæ'sia, Sm. p. 7. Casions Meadow-grass.

compress'a, L. 69. p. 6,7. Flat-stemmed $M$.

v. sub'compress'a (Parn.)

v. polyno'da ${ }^{4}$ (Parn.)

decumb'ens, With. = Siegling'ia decumb'ens, Bernh.

dist'ans, Sm. = Glycer'ia dist'ans, Wahl.

flexuo'sa, Sm. = P. lax'a, Hænke.

flu'itans, Scop. = Glycer'ia flu'itans, R.Br.

glauc'a, Sm. $10 .{ }^{5}$ p. 6, 7. Glaucous Meadow-grass.

lax'a, Hænke. 3. p. 7, 8. Wavy Meadow-grass.

lǒlia'cea, Huds. = Festu'ca lŏlia'cea.

marit'ima, Huds. = Glycer'ia marit'ima, Wahl.

min'or, Bab. = P. lax'a, Hænke.

monta'ua, Parn. Included in P. Balfour'ii, Bab.

nem'ora'lis, L. 82. p. 6, 7. Wood Meadow-grass.

v. vulga'ris, Gaud.

v. giaucanth'a, Reichb.

v. Parnell'ii, ${ }^{7}$ Hook. \& Arn.

v. di'vārica'ta, Syme.

Parnell'ii, Bab. = P. nem'ora'lis, v. Parnell'ii.

polyno'da, Parn. $=$ P. compress'a, v. polyno'da.

pratens'is, L. 108. p. 6, 7. Sinooth Meadow-grass.

v. sub'cærul'ea $^{8}(\mathrm{Sm}$.)

v. angust'ifol'ia (L.)

v. strigo'sa (Gaud.)

procumb'ens, Curt. = Festu'ca procumb'ens, Kunth.

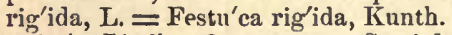

strict'a, Lindb. 3. p. 9, 10. Straight-stemmed Meadow-grass. sub'cærul'ea, Sm. = P. pratens'is, v. sub'cærul'ea.

sude'tica, Hænke. p. Hooded-leaved Meadow.grass.

trivia'lis, L. 108. p. 6, 7. Rough Meadow-grass.

v. Kœle'ri (DC.)

v. parviflo'ra, Parn.

Polemo'nia'Ces, Juss. Phloxworts.

$\left|\begin{array}{l}\text { L.C. } \\ 1168 \\ 1166 \\ 1166 \mathrm{~b} \\ 1163 \\ 1163 \mathrm{~b} \\ 1167 \\ 1167 \mathrm{~b}\end{array}\right|$

1164

934

1702

1700

1706

1701

$170 \mathrm{~s}$

$1708 b$

$1708 \mathrm{c}$

.

1705

1704

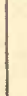

1707

$1707 a$

$1707 \mathrm{~b}$

$1707 \mathrm{c}$

$1707 d$

1710

$1710 \mathrm{~b}$

$1710 \mathrm{c}$

$1710 \mathrm{~d}$

1703

1709

1711

$171 \mathrm{lb}$

$1711 \mathrm{c}$

981 vi.922
.1167

vii. 1166

vii. 1163

$B$.

829

827

825

828

826

1241

1235

1228

xi. 1767

xi. 1761

xi. 1765

xi. 1770

1242

1236

1231

1229

xi. 1766

$x i .1764$

1240

1234

1230

xi. 1768

1239

xi.1769

xi.1771

1237

xi.1772

1232

1233

xi. 1763

xi. 1773

1238

1 Star-of-the-East. 2 Plantago Timbali. 3 Including Poa glauea. 4 P. polynoda.

P. Balfonrii. 6 P. flexuosa.-P. minor. 7 P. Parnellii. 8 P. subcerulea. 
Pŏlemo'nium, Tourn. Pŏ́емo' Nia'Cex, Phloxworts. Pent. Mon. cærul'eum, L. 5. p. 6, 7. Blue Jacob's Ladder. Greek Valorian.

Polycarp'on, L. CAR'YOPHYLL'Es, Cloveworts. Tri. Trig. tet'raphyll'um, L. 4. a. 6, 7. Four-leaved Allseed or Polycarp.

Polyg'ala, L. Polyand'ese, Milkworts. Diad. Oct.

ama'ra, L. 2. p. 6, 7. Small Bitter Milkwort.

v. üli'gino'sa, ${ }^{1} \mathrm{Fr}$.

v. austria'ca, ${ }^{2}$ Crantz.

austria'ca, Crantz. $=P_{\text {. ama'ra, }} \mathbf{v}$. austria'ca.

calca'rea, F. Schultz. 15. p. 6,7. Chalk Milkwort.

depress'a, Wend. $=$ P. serpylla'cea, Weihe.

oxypt'era, ${ }^{3}$ Reichb. 24. p. 6-8.

serpylla'cea, Weihe. 74. p. 6-8. Lesser Common Milkwort. v. cil'ia'ta, Lebel.

ùli'gino'sa, Reichb. $=$ P. ama'ra v. ũli'gino'sa.

vulga'ris, L. 59. p. 6-8. common Milkwort.

v. grandifo'ra, Bab.

v. oxypt'era $=$ P. oxypt'era, Reichb.

Polygal'en, Juss. Milkworts.

Pon'YGona'ce.e, Juss. Buckwheats.

Pol'ygon'ătum, Tourn. LI'LIA'CER, Lilyworts. Hex. Mon. multiflo'rum, ${ }^{5}$ All. 32. p. 5, 6. Common Soloman's Seal. offic'ina'le, ${ }^{6}$ All. 14. p. 5, 6. Angular-stemmed S.S. vert'icilla'tum, ${ }^{7}$ All. 4. p. 6, 7. Whorled, or Narrow-leaved S.S.

Polyg'onum, L. Poz'YGONA'CE. Buckwheats. Oct. Trig. amphib'ium, L. 105. p. 7, 8. Amphibions Bistort. ${ }^{8}$

$\checkmark$. terrest're, Leers.

v. na'tans.

avic'ula're, L. 110. a. 5-10. Common Knot-grass.

v. agresti'num ( $\mathrm{J}$ ord.)

v. vulga'tum, Syme.

v. ar'ênast'rum (Bor.)

v. mi'crosperm'um (Jord.)

v. ru'rivag'um (Jord.)

v. littora'le (Link)

Bistort'a, L. 70. p. 6-9. Common Bistort. Snake-weed. ${ }^{9}$

Convolv'ulus, L. 107.a.7-9. Climbing Buckwheat. Black Bindweed. v. pseud'o-dumeto'rum, H. C. Wats.

du'meto'rum, L. 11. a. 7, 8. Copse Buckwheat.

Fa'gopy'rum, L. = Fa'gopy'rum es'culent'um, Mœench.

Hrdrop'iper, L. 104. a. 8, 9. Water Pepper. Biting Persicaria. lap'athifol'ium, L. 97. a. 7-9. Glandular Pale-flowered $P$. v. nodo'sum $=$ P. macula'tum, Dyer \& Trim. lax'um, Bab. = macula'tum, Dyer \& 'Trim.

macula'tum, ${ }^{10}$ Dyer and Trim. 26. a. 7-9. Slender-headed $P$. marit'imum, L. 4. a. b. or s. $7-9$. Sea-side Knot-grass. min'us, Huds. 48. a. 8, 9. Small, Slender, or Creeping $P$. mi'te, Schrank. 19. a. 8, 9. Lax-flowered Persicaria. nodo'sum, Pers. $=$ P. P'ersica'ria, v. ela'tum.

Persica'ria, L. 112, a. 7-10. Spotted Persicaria. v. ela'tum, Gr. et Godr.

Ra'ii, Bab. = P. Robert'i, Loisel.

Robert'i, ${ }^{11}$ Loisel. 36. a. or b. 8, 9. Ray's Knot-grass. vivip'arum, L. 28. p. 6-8. Viviparous Alpine Bistort.

Polypod'ium, L. Fit'ices, Ferns. Crypt. Fil.

alpest're, Hoppe = Athyr'ium alpest're, Milde.

calca'reum, Sm. = Phègopt'eris Robert'ia'na, A.Br.

Dr'yopt'eris, L. = Phégopt'eris Dry̌opt'eris, Fée.

\begin{tabular}{|c|c|c|}
\hline $\begin{array}{l}\text { L.C. } \\
981\end{array}$ & $\begin{array}{c}\text { E.B. } \\
\text { vi. } 922\end{array}$ & $\begin{array}{l}\text { B. } \\
682\end{array}$ \\
\hline 252 & ii. 258 & 171 \\
\hline 181 & & \\
\hline $\begin{array}{l}181 \mathrm{a} \\
181 \mathrm{~b}\end{array}$ & (ii. 189) & \\
\hline 180 & $\begin{array}{l}\text { ii. } 189 \\
\text { ii. } 188 \\
\text { ii. } 187\end{array}$ & \\
\hline 178 & (ii. 186) & \\
\hline $\begin{array}{l}179 \\
179 b\end{array}$ & (ii.187) & \\
\hline 177 & ii. 185 & 125 \\
\hline & ii. 186 & \\
\hline 177 & ii. 185 & \\
\hline 1205 & viii. 1210 & \\
\hline 1392 & ix.1513 & 1026 \\
\hline 1393 & ix.1512 & 1027 \\
\hline 1391 & ix.1511 & 1025 \\
\hline 1216 & viii. 1241,2 & 872 \\
\hline $1216 \mathrm{~b}$ & $\begin{array}{l}\text { viii. } 1241 \\
\text { viii.1242 }\end{array}$ & \\
\hline 1207 & viii.1229-31 & 866 \\
\hline $1207 a$ & & \\
\hline $\begin{array}{l}1207 \mathrm{~b} \\
1207 \mathrm{c}\end{array}$ & viii. 1229 & \\
\hline $\begin{array}{l}1207 \mathrm{c} \\
1207 \mathrm{~d}\end{array}$ & viii. 1230 & \\
\hline $\begin{array}{l}1207 \mathrm{~d} \\
1207 \mathrm{e}\end{array}$ & viii. 1231 & \\
\hline $1207 \mathrm{f}$ & & \\
\hline 1217 & viii.1243 & 871 \\
\hline 1205 & viii.1227 & 868 \\
\hline $1205 \mathrm{~b}$ & & \\
\hline 1206 & $\begin{array}{l}\text { viii. } 1228 \\
\text { viii. } 1226\end{array}$ & $\begin{array}{l}869 \\
\cdot\end{array}$ \\
\hline 1210 & viii. 1234 & 875 \\
\hline 1214 & $\begin{array}{l}\text { viii.1239 } \\
\text { viii.1240 }\end{array}$ & 874 \\
\hline 1215 & (viii.12 40 ) & \\
\hline 1209 & viii. I 233 & 867 \\
\hline 1211 & viii. 1235 & 876 \\
\hline 1212 & riii.1236 & \\
\hline 1213 & viii. 1237 & 873 \\
\hline $1213 b$ & viii. 1238 & \\
\hline 1208 & $\begin{array}{l}\text { viii.1232 } \\
\text { (viii.1 232) }\end{array}$ & \\
\hline 1218 & viii.124t & 870 \\
\hline & & 1274 \\
\hline & & 1275 \\
\hline
\end{tabular}

4 P. depressa. 5 Con-

1 Polygala nliginosa. vallaria multiflors.

2 P. sustriacs.

$3 \mathrm{P}$. vulgaris, $\mathrm{v}$, oxyptera. 7 C. verticillata.

81

69

875

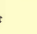

10 Polygonum laxum.-P. lapathifolium, v, nodosum. 11 P. Raii. 
Polypod'ium, L.-continued.

flex'ile, Moore = Athyr'ium flex'ile, Syme.

hyp'erbor'eum, Sw. = Woods'ia byp'erbor'ea, R.Br.

Lonchi'tis, L. = Polystich'um Lonchi'tis, Roth.

Oreopt'eris, Ehr. = Lastræ'a Óreopt'eris, Presl.

Phēgopt'eris, L. = Phēgopt'eris polypod'ioi'des, Fée.

Robert'ia'num, Hoffm. = Phēgopt'eris Robert'ia'na, R.Br.

Thëlypt'eris, L. = Lastræ'a 'l'hēlypt'eris, Pres].

vulga're, L. 112. p. 6-9. Common Polypody.

v. serra'tum, Willd.

v. camb'ricum, Willd.

Polypo'gon, Desf. Grämin'es, Grasses. Tri. Dig.

littora'lis, ${ }^{1} \mathrm{Sm}$. 6. a. 7. Perennial Beard-grass.

mons'peliens' is, ${ }^{2}$ Desf. 6. a. 6, 7. Annual Beard-grass.

Polystich'um, Roth. Fin'ices, Ferns. Crypt. Fil.

acu'lea'tum, Roth = P. loba'tum, Presl.

angula're, ${ }^{3}$ Presl. 63. p. 7, 8. Angular-leaved Shield-fern.

v. hastula'tum, Kunze.

v. ala'tum, Moore.

v. grac'ile, Wollaston.

loba'tum, ${ }^{4}$ Presl. 100. p. 7, 8. Hard Holly Fern.

v. genui'num, ${ }^{6}$ Syme. Close-leaved prickly Shield-fern.

v. acu'lea'tum, Syme.

\begin{tabular}{l|c|c} 
L.C. & E.B. & B. \\
& & \\
& & 1273 \\
1801 & xii.1842 & 1272 \\
$1801 \mathrm{~b}$ & & \\
$1801 \mathrm{c}$ & & \\
1659 & xi.1714 & 1175 \\
1658 & xi.1713 & 1174 \\
1790 & xii.1861 & \\
$1790 \mathrm{~b}$ & & \\
$1790 \mathrm{c}$ & & \\
$1790 \mathrm{~d}$ & & \\
1789 & xii.1860 & $(1279)$ \\
$1789 \mathrm{a}$ &
\end{tabular}

Po'pulus, Tourn. SAL'ICIN'Ee, Willowworts. Diœc. Uct.

alb'a, L. 60. t. 3, 4. White Poplar. Abele Tree.

canesc'ens, Sm. 43. t. 3, 4. Grey or Hoary Poplar.

nigra, L. t. 3. Black Poplar.

trem'ula, L. 100. t. 4. Aspen. Trembling Poplar.

v. villo'sa (Lange)

v. gla'bra, Syme.

Portula'cere, Juss. Purslanes.

Pot'amoge'tōn, L. NA'1AdA'CEe, Naiads. Tetran. Tetrag. acu'tifol'ius, Link. 12. p. 6-8. Sharp-leaved Pondweed.

v. ma'jor, Fieber.

compress'us, $\mathrm{Sm}_{.}=$P. Fries'ii, Rupr.

cor'ia'ceus, Nolte.

crisp'us, L. 92. p. 7, 8. Curled Pondweed.

v. serra'tus (Huds.)

decip'iens, ${ }^{7}$ Nolte. 12. p. 7.

dens'us, L. 57. p. 7-9. Opposite-, or Close-leaved Pondweed.

filliform'is, Nolte. 12. p. 7-9. Slender-leaved Pondweed.

fla'bella'tus, Bab. 37. b. 6, 7. Fan-like Pondweed.

Hu'itans, Roth. 1.

flu'itans, Sm. $=$ P. rufesc'ens, Schrad.

Fries'ii, ${ }^{8}$ Rupr. 34. p. 6, 7. Flat-stemmed Pondweed.

grāmin'eus, Sm. = P. obtu'sifol'ius, Mert. \& Koch.

Griffith'ii, Ar. Benn. 1. p. 7.

$1789 \mathrm{a}$

$1789 \mathrm{~b}$

1788

xii.1859

(1278)

1311

viii. 1299

1312 viii. 1300

1314 viii. 1302

1313 viii. 1301

13 I3a

$1313 \mathrm{~b}$

253

ii. 259

1499

$1499 \mathrm{~b}$

ix. 1416

961

1496

ix. 1413

958

(ix.1409)

1497 ix.1414

1507

ix. 1424

1506

ix. 1421

1481

1501

(ix.1418)

het'erophyll'us, Schreb. 66. p. 6-9, Various-leaved Pondweed.

1493

v. max'imus, Ar. Benn.

Kirk'ii, Syme = P. sparg'anifol'ius, Læstad.

lanceola'tus, Sm. 2. p. 7-9. Lanceolate Pondweed.

Lonchi'tes, 'Tuck. I. p. 6-9. Willow-leaved Pondweed.

1487

ix. 1406

954

longifol'ius, Bab. $=$ P. lu'cens, v. longifol'ius.

lu'cens, ${ }^{9}$ L. 73. p. 6-9. Great Pondweed.

v. acu'mina'tus (Schum.)

v. longifol'ius, ${ }^{10} \mathrm{Bab}$. (non Gay) I.

v. decip'iens $=$ P. decip'iens, Nolte.

486 ix. 1405

1485 ix. 1404

ix. 1410

1489

ix. 1408

955

1 Agrostis littoralis, 2 A. panicea. 3 Aspidium angulare. 1 A. aculeatum.-Polystichum aculeatum. 5 Aspidium lobatum. 6 A. Lonchitis.-Polypodium Lonchitis.

8 P. compressus.-P. mucronatus. 9 P. Proteus, 10 P. longifolius. 
Pot'amoge'tōn, $\mathrm{L}_{\mathrm{L}}-$ continued.

mu'crona'tus, Schrad. = P. Fries'ii, Rupr.

na'tans, L. 79. p. 6-9. Floating Pondweed. ${ }^{1}$

\section{1}

nit'ens, Web. 15. p.9. Shining Pondweed.

v. curvifol'ius, Hartm.

oblong'us, Viv. = P. polygo'nifol'ius, Pourr.

obtu'sifol'ius, ${ }^{2}$ Mert. \& Koch. 53. p. 7, 8. Grassy Pondweed.

v. fluvia'lis, Lange et Mort.

pectina'tus, L. 74. p. 6-8. Fennel-leaved Pondweed.

v. scopa'rius, Wallr.

per'folia'tus, L. 89. p. 6-9. Perfoliate Pondweed.

v. Jackso'nii, F. A. Lees.

plantāgin'eus, Du Croz. 30. p. 6-9. Plantain-leaved $P$.

polygo'nifol'ius, ${ }^{3}$ Pourr. 99. p. 6-9. Oblong-leaved $P$.

r. pseud'o-flu'itans, Syme.

v. linea'ris, Syme.

prielong'us, Wulf. 33. p. 5-7. Long-stalked Pondweed.

Pro'teus, Schlecht. $=$ P. lu'cens, L.

pusill'us, L. 95. p. 7, 8. Small or Slender Pondweed.

r. tenuiss'imus, Koch.

v. rig'ida, Ar. Benn.

rufesc'ens, ${ }^{4}$ Schrad. 62. p. 7-9. Reddish or Long-leaved Floating $P$.

$v$. spăthula tus (Koch et $\mathrm{Ziz}$ )

sal'icifol'ius, Wolfg. 1. p. 7.

sparg'anifol ius, ${ }^{5}$ Lrestad. I. p. 7. Ribbon-leaved Pondweed.

Sturrock'ii, Ar. Benn. 1.

trǐchoïdes, Cham. 4. p. 7, 8. Hair-leaved Pondweed.

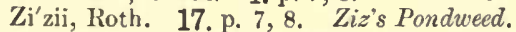

zoste'ræefol'ius, Schum. 19. p. 6-8. Grass-uracked-lectved $P$.

Pot'entill'a, L. RosA'ces, Roseworts. Icos. Pol.

alb'a, L. p. 6-9. White-rock Cinquefoil.

alpest'ris, Hall. f. = P. macula'ta, Pourr.

Anseri'na, L. 112. p. 6-8. Silverweed. Gooseweed. ${ }^{B}$

argent'ea, L. 57. p. 6, 7. Hoary Cinquefoil.

aur'ea, Sm. non L. $=$ P. macula'ta Pourr.

Com'arum, ${ }^{7}$ Nestl. [palust'ris, Scop.] 99. p.6,7. Marsh Cinquefoil.

Fraga'ria, DC. = P. Fraga'riast'rum, Ehrh.

Fraga'riast'rum, ${ }^{8}$ Ehrh. 101. p. 3-5. Barren Strawberry. ${ }^{\circ}$

frutico'sa, L. 4. s. 6, 7. Shrubby Cinquefoil.

hirt' $a$, L. p. 6.

intermed'ia, Nesl. = P. opa'ca, Sm.

macula'ta, ${ }^{10}$ Pourr. [rub'ens, Vill.] 14. p. 6, 7. Yellow Alpine $C$. nem'ora'lis, Nestl. = P. procumbens, Sibth.

norve'gicu, L. p. 7.

opa'ca," Sm. p. 6. Saw-leaved Hairy Cinquefoil.

palust'ris, Scop. See Com'arum, Nestl.

procumb'ens, ${ }^{12}$ Sibth. 69. p. 6, 7. Creeping Tormentil.

rept'ans, L. 89. p. 6-8. Creeping Cinquefoil.

rub'ens, Vill. See macula'ta, Pourr.

rupest'ris, L. 1. p. 5, 6. Strawberry-flowered or Rock $C$.

sal'isburgens'is, Hænke $=$ P. macula'ta, Pourr.

Sibbald'i, ${ }^{13}$ Hall. f. 18. p. 7. Proctmbent Sibbaldia.

sylvest'ris, Neck. See 'Tormentill'a, Neck.

Tormentill'a, ${ }^{14}$ Neck. [sylvest'ris, Neck.] 112.p.6-9. Tormentil. ${ }^{15}$ trid'enta'ta, Sm. p. 5, 6. Three-toothed or Trifid Cinquefoil. vern'a, L. 22. p. 4-6. Spring Cinquefoil.

$1505 \mathrm{~b}$
L.C.

1479

$1479 b$

1488 ix. 1407

E.B.

ix. 1418

ix.1399

B.

953

$1488 \mathrm{~b}$

1500 ix. 1417

$1500 \mathrm{~b}$

1505

1495

$1495 \mathrm{~b}$

1482

1480

$1480 \mathrm{~b}$

$1480 \mathrm{c}$

1494

1502

$1502 \mathrm{~b}$

$1502 \mathrm{c}$

1483

$1483 \mathrm{~b}$

1492

1484

1503

1504

1491

1498

ix. 1422

ix. 1423

ix. 1412

ix. 1401

ix. 1400

ix. 1411

956

ix. 1419

962

ix. 1402

ix. 1403

ix. 1420

ix. 1415

iii. 429

491

iii. 433

493

iii. 435

495

iii.437

317

314

319

485

iii. 427

311

494

iii. 436

316

484

487

(iii.429)

483

489

iii. 431

490 iii. 432

312

492

iii. 434

318

496

iii. 426

iii. 430

488 (iii.430)

486

iii. 428
1 Sharp-fruited Broad-Laved Fondweed. 4 P. fluitans, \$m. 5 P. Kirkii. 6 Wild Tansy. sterilis. Strauberry-leaved Cinquefoil.

2 Potamogeton gramineus. 
Pote'rium, L. Rosa'ces, Roseworts. Monœc. Pol. mu'rica'tum, Spach [polyg'amum, Waldst. \& Kit.] p. 7. Muricated Salad Burnet. ${ }^{2}$

offic'ina'le, ${ }^{2}$ Hook, f. 62. p. 6-8. Great Burnet. Sanguisorb. polyg'amum, Waldst. \& Kit. See mu'rica'tum, Spach.

Sanguisorb'a, L. 70. p. 6-8. Salad Burnet. Garden B.

Prēnanth'es, Gærtn. Compos'its, Composites. Syn. Mon. hiera'cifol'ia, Willd. = Cre'pis pulchr'a, $\mathrm{L}$.

mura'lis, L. = Lactu'ca mura'lis, Fresen. purpur'ea, L. p. 8.

Pri'mula, L. Prímula'ces, Primworts. Pent. Mon. ela'tior, Jacq. 5. p. 4, 5. Jacquin's Oxlip.

farino'sa, L. 11. p. 6, 7. Bird's-eye Primrose. Mealy $P$. hyb'ridæ :-
a. ve'ris $\times$ vulga'ris, ${ }^{3}$ Common Oxslip.
b. ve'ris $\times$ ela'tior.
c. vulga'ris $\times$ ela'tior.

officina'li-vulga'ris $=P$. ve'ris $\times$ vulga'ris.

offic'inna'lis, $L$. $=$ P. ve'ris, $L$.

sco'tica, Hook. 4. p. 6-9. Scottish Bird's-eye Primrose.

v. acau'lis, Iond. Cat.

ve'ris, ${ }^{4}$ L. 89. p. 4, 5. Cowslip. Paigle.

vulga'ris, Huds. 111. p. 4, 5. Common Primrose.

v. acau'lis (Hill)

v. canlesc'ens (Koch)

Prímula'ces, Vent. Primworts.

Pris'matocarp'us, L'Hérit.

hyb'ridus, L'Hérit. = Specula'ria hyb'rida, DC.

Prunell'a, L. LAn'1a't

vulga'ris, ${ }^{6}$ L. 112. p. 7-9. Self-heal. Slough-heal.

Pru'nus, L. Rosa'CEd, Roseworts. Icos. Mon.

Av'ium, ${ }^{6}$ L. 90. t. 5. Wild Cherry. Gean-tree.

Cer'asus, ${ }^{7}$ L. 33. s. 5. Dwarf Cherry. Morello Cherry.

commu'nis, ${ }^{8}$ Huds. 103. s. 4, 5. Blackthorn. Sloe.

v. frut'icans (Weihe)

domest'ica, L. t. 5. Wild Plum.

insitit'ia, L. 59. s. 4. Bullace-tree.

Pad'us, ${ }^{9}$ L. 58. t. 5. Bird Cherry.

spino'sa, L. $=$ P. commu'nis, Huds.

Psamm'a, Roem. \& Schult.

ar'éna ria, Roem. \& Schult. = Ammoph'ila arund'ina'cea, Host.

balt'ica, Roem. \& Schult. = Ammoph'ila balt'ica, Link.

Pseud'othyr'ium, Newm.

alpest're, Newm. = Athyr'ium alpest're, Milde.

flex'ile, Newm. = Athyr'ium flex'ile, Syme.

Pter'is, L. FiL'ices, Ferns. Crypt. Fil. aquili'na, L. 112. p. 7, 8. Brake Fern. Bracken. Brakes. crisp'a, L. = Crypt'ogramm'a crisp'a, R.Br.

Pu'lica'ria, Gærtn. Coм Pos'rt s, Composites. Syn. Sup. dys'enter'ica, ${ }^{10}$ Gærtn. 76. p. 7-9. Greater Fleabane. vulga'ris, ${ }^{11}$ Gærtn. 25. a. 8, 9. Lesser Fleabane.

Pul'mona'ria, 'Tourn. BorĀGIN'Es, Borageworts. Pent. Mon. angust'ifol'ia, L. 4. p. 4-6. Narrow-leaved Lungwort. marit'ima, L. $=$ Mertens'ia marit'ima, Gray. offic'ina'lis, L. p. 4-6. Lungwort. Jerusalem Cowslip.

Pyreth'rum, L. in'odo'rum, Sm. = Ma'trica'ria in'odo'ra, L.

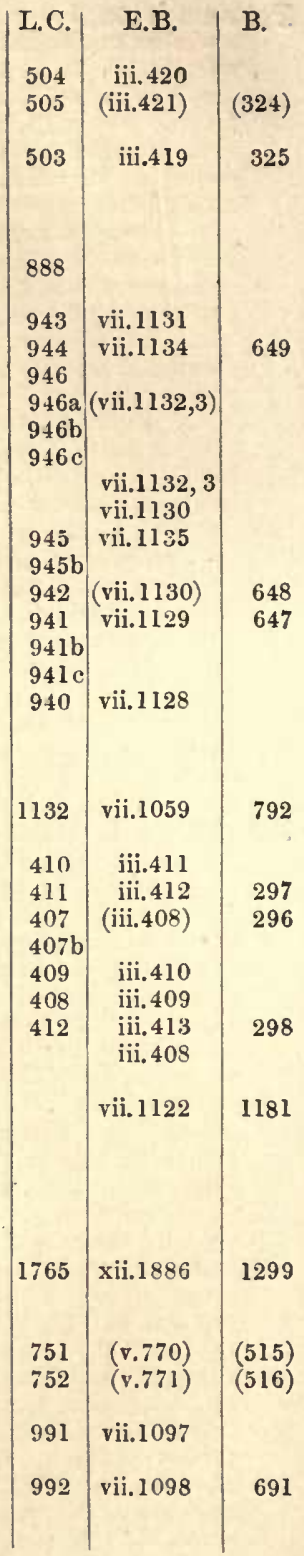

I Rough-seeded or Lesser Burnet.

4 P. officinalis. 5 Brnnella vulgaris.

9 Cerasus Padns. 10 Inula dysenterica.
2 Sanguisorba officinalis. 6 Cerasus avium. 7 C. austera.

3 Primula officinali-vulgaris. II I. pulicaria. 
Pyreth'rum, L.-continued.

marit'imum, Sm. = Ma'trica'ria in'odo'ra, v. marit'ima.

Parthen'ium, Sm. = Chrysanth'emum Parthen'ium, Pers.

Pyr'ola, 'Tourn. ER'îca'cex, Heathworts. Dec. Mon. med'ia, Sw. 37. p. 7, 8. Intermediate Wintergreen. min'or, ${ }^{1}$ L. 65. p. 6-8. Lesser Wintergreen. ros'ea, $\mathrm{Sm} .=\mathrm{P}$. inin'or, L.

rotund'ifol'ia, L. 21. p.7-9. Round-leaved Wintergreen. Larger W.

v. marit'ima, ${ }^{2}$ Kenyon.

v. ar'êna'ria, Koch = v. marit'ima, Kenyon.

secund'a, L. 18. p. 7. Serrated Wintergreen.

a'niflo'ra, $\mathrm{L}$. = Mone'ses grandiflo'ra, Gray.

Pyr'us, L. Rosa'ce.e, Roseworts. Icos. Mon. acerb'a, DC. $=$ P. ma'lus, v. acerb'a.

$A^{\prime}$ chras, Boreau $=$ P. commu'nis, v. A'chras.

Ar'ia, Sm. 46. t. 5, 6. Common White-beam.

v. rupic'ola, ${ }^{3}$ Syme. Rock White-beam.

Aucupa'ria, ${ }^{4}$ Gærtn. 106. t. 5, 6. Mountain-Ash. Rowan-tree. commn'nis, L. 49, t. 4, 5. Wild Pear-tree.

v. Py̆rast'er, ${ }^{5}(\mathrm{~L}$. $)$

v. $A^{\prime} \operatorname{chras}^{6}$ (Gærtn.)

$\checkmark$. corda'ta (Desv.) Small-fruited Pear.

corda'ta, Desv. = P. commu'nis, v. corda'ta.

domest'ica, Sm. t. 5. True or Common Service-tree.

fenn'ica, Bab. = P. hyb'rida, L.

germa'nica, ${ }^{8}$ L. t. 5, 6. Wild Medlar.

hyb'rida, ${ }^{9}$ L. 7. s. 7. Bastard Mountain-Ash.

intermed'ia. Ehrb. $=$ P. scand'ica, Syme.

la'tifol'ia, Syme. 9. t. 5.

Ma'lus, L. 84. t. 5. Wild Apple-tree. Crab-tree.

v. acerb'a, ${ }^{10}$ (DC.) t. 6. Common Crab-apple.

v. mi'tis, ${ }^{1 i}$ Wallr. t. 7. Wild Apple.

mi'tis, Syme = P. Ma'lus, v. mi'tis.

pinuātif'ida, Ehr. = P. hyb'rida, L.

Py̆rast'er, Bor. $=$ P. commu'nis, v. Py̆rast'er.

rupic'ola, Syme $=P$. Ar'ia, v. rupic'ola.

scand'ica, ${ }^{12}$ Syme. 3, t. 5. Lobed-leaved White-beam.

se'mipinna'ta. Roth's Bastard Mountain-Ash.

tormina'lis, ${ }^{13}$ Ehrh. 47, t. 4, 5. Wild Service-tree.

Querc'us, Tourn. Cu'Puliz'ere. Monoc. Pol.

pedunc'ula'ta, Willd. $=Q$. ro'bur, v. pedunc'ula'ta.

Ro'bur, L. 105. t. 4, 5. The Oak.

v. pedunc'ula'ta ${ }^{14}$ (Ehrh.) 67. Common Oak.

v. intermed'ia (D. Don)

v. sessiliflo'ra ${ }^{15}$ (Salisb.) 65. Sessile-fruited Oak. sessiliffo'ra, Salisb. $=$ Q. Ro'bur, v. sessiliflo'ra.

Rad'iola, Dill. Lr'vex, Flaxworts. Tetran. Tetrag.

linoi'des, ${ }^{16}$ Roth. 82. a. 7, 8. Thyme-leaved Flax-seed. All-seed. Mill'egra'na, $\mathrm{Sm} .=\mathrm{R}$. linoï'des, Roth.

RanUNo'CLA'CE.E, Juss. Crowfoots.

Ranunc'ulus, L. Randeciula'ce.s, Croufoots. Polyan. Polyg. a'cris, L. 111. p. 4-9. Upright Meadow Crowfoot. King-cup.

v. tomophyll'us (Jord.)

v. rulga'tus (Jord.)

v. Boræa'nus (Jord.)

L.C. E.B.

B.

.

929

vi. 897

642

930

vi. 898

643

928 vi.895

641

$928 \mathrm{~b}$ (vi.896)

vi.896

931

vi.899

vi. 900

644

640

iii. 489

iii. 488

525 iii.482

525 b (iii. 483)

530 iii.486

531 iii.488

531a (iii.488)

531 b (iii. 488)

$531 \mathrm{c}$

529

iii. 487

iii. 485

533

(iii.478)

528 (iii.485)

526

532 iii. 489,90

532a (iii.489)

$532 \mathrm{~b}$ (iii. 490)

iii.490

iii. 488

iii. 483

527

iii.484

524

iii.481

335

viii. 1288

1277 viii. $1288,9 \quad 914$

1277 a (viii. 1288)

$1277 \mathrm{~b}$

1277 c (viii. 1289)

viii. 1289

286 (ii.288)

(192)

ii. 288

192

1

i. 1

29

i. 33

17

1 Pyrola rosea. 2 Pyrus rotundifolia, v. arenaria. 3 P. rupicola. Pyraster. 6 P. Achras. 7 P. cordata. 8 Mespilug germanica. 9 Pyrus fennica. - P. piunatifida.

4 Sorbus Ancuparia. 5 Pyras 15 Q. sessiflora. 16 Radiola Millegrana. 
Ranunc'ulus, L.-continued.

alpest'ris, L. p. 5. Alpine White Crowfoot.

aqua'tilis, $\mathbf{L}$.

arvens'is, L. 66, a. 5.7. Corn Crowfoot.

auric'omus, L. 85. p. 4, 5. Wood Crowfoot. Goldilocks. ${ }^{1}$

Baudo'tii, Godr. 37. a. or p. 5-8. Baudot's Water-Crowfoot.

v. vulga'ris.

v. confu'sus $^{2}$ (Godr.)

v. mari'nus ${ }^{3}$ (Arr. \& Fr.)

bulbo'sus, L. 97. p. 5-7. Bulbous-rooted Crowfoot. Buttercup. chærophyll'us, L. C. p. 5. Chervil-leaved Buttercup.

circina'tus, Sibth. 53. p. 6-8. Rigid-leaved Water-Crowfoot. cœno'sus, Guss. $=\mathrm{R}$. hedera'ceus, $\mathrm{L}$.

confu'sus, Godr. $=$ R. Baudo'tii, $v$. confu'sus.

divers'ifol'ius, Gilib. = R. het'erophyll'us, Web.

Drouet'ii, Godr. 49. p. 5, 6. Drouet's Water-Crowfoot.

v. Godro'nii (Gren.)

v. confervoï'des (Fr.)

Fica'ria, ${ }^{4}$ L. 105. p. 3-5. Lesser Celandine. Pilewort Crowfoot. ${ }^{5}$ v. incumb'ens, F. Schultz.

[flabella'tus, Desf.]

v. europæ'us, Nyman. C.

Flamm'ula, L. 112. p. 6-8. 'Lesser Spearwort.

v. pseud'o-rept'ans, Syme.

flo'ribund'us, Bab. $=$ R. pelta'tus, v. flo'ribund'us.

flu'itans, Lam. 51. p. 6-8. River or Floating Water-Crowfoot. v. Ba'chii (Wirtg.)

grämin'eus, L. p. 5, 6. Grass-leaved Crowfoot.

hědera'ceus, ${ }^{6}$ L. 101. p. 5-8. Ivy-leaved Water-Crowfoot. ${ }^{7}$ v. omi'ophyll'us ('Ten.)

het'erophyll'us, ${ }^{8}$ Web. ex p. 5, 6 .

hirsu'tus, Curt. $=$ R. sardo'us, Crantz.

intermed'ius, Hiern. $=$ R. triparti'tus, DC.

Lenormand'i, F. Schultz. 48. p. 6-8. Lenormand's Water-CrowLing'ua, L. 78. p. 7-9. Greater Speamort.

mari'nus, Fr. = R. Baudo'tii, v. mari'nus.

[wort.

oph'ioglo'ssifol'ius, Vill. 1. a.6-8. Adder's-tongue-leaved Spearpant'othrix, DC. p. 5-8. Small Water-Crowfoot.

parviflo'rus, L. 57. a. 5-8. Small-flowered Crowfoot.

pelta'tus, Schrank. 69. p.5-9. Common Water-Crowfoot.

v. vulga'ris.

v. trunca'tus, Hiern.

v. flo'ribund'us ${ }^{9}$ (Bab.)

v. pe'nicilla'tus, ${ }^{10}$ Hiern.

pe'nicilla'tus, Dum. $=\mathrm{R}$. pelta'tus, v. pe'nicilla'tus.

Phil'ono'tis, Ehrh. = R. sardo' us, Crantz.

pseud'o-flu'itans, Bab.

v. submers'us, Hiern (form)

re'pens, L. 111. p. 5-8. Creeping Crowfoot.

rept'ans, L. 3. p. 8 . Creeping Spearwort.

sardo'ns, ${ }^{11}$ Crantz. 74. a. or b. 6-10. Palc Hairy Crowfoot.

v. parv'ulus (L.)

scèlera'tus, L. 95. a. or b. 5-9. Celery-leaved Water Crowfoot. trichophyll'us, Chaix. 45. p. 5, 6. Hair-leaved $W . C .^{12}$ triparti'tus, ${ }^{13}$ DC. 10. a. or p. 5-7. Three-leaved W. $C$. v. intermed'ins (Knaf.)

triphyll'os, Wallr. C. p. 5-7. 
Raph'anus, L. Crucif'Ere, Cmucifers. Tetrad. Siliq. marit'imus, Sm. 22. b. 7, 8. Sea Radish.

Raphanist'rum, L. 105. a. 5-9. Wild or Field Radish.'

3

al. $b^{\prime} a,{ }^{2}$ L. b. or p. 7, 8. Upright or White Mignonette.

frut'iculo'sa, L. = R. alb'a, L.

lu'tea, L. 53. b. or p. Wild, Yellow, or Cut-leaved M.4

Lu'teola, L. 94. b. 6-8. Weld. Dyer's Rocket. Yellow-weed. ${ }^{5}$ suffrut'iculo'sa, L. $=$ R. alb'a, L.

REs'EDA'CEx, DC. Weldworts.

RHAM'NeE, Juss, Rhamnads.

Rham'nus, L. RHAN'xes, Rhamnads. Pent. Mon. cathart'icus, L. 55. s. 5-7. Purging Buckthorn.

Frang'ula, L. 58. s. 5, 6. Berry-bearing Alder. ${ }^{6}$

Rhinanth'us, L. Scro'Phularin'ex, Figworts. Did. Ang. angust'ifol'ius, Gmel. $=$ R. ma'jor, v. stenopt'era.

Crist'a-gall'i, ${ }^{7}$ L. 112. a. 5-7. Common Yellow-Rattle. ma'jor, Ehrh. 20. a. 7, 8. Larger Yellow.Rattle.

v. platypt'era, Fr.

v. stenopt'era, ${ }^{8} \mathrm{Fr}$.

v. apt'era, Fr.

min'or, Ehrh. = R. Crist'a-gall'i, L.

Rhod'iola, L.

ros'ea, $\mathrm{L} .=$ Sed'um Rhod'iola, DC.

Rhȳncosp'ora, Vahl. Crp'ers'cexe, Sedges. Tri. Mon.

alb'a, Vahl. 75. p. 6, 7. White Beak-Sedge.

fusc'a, ${ }^{10}$ Roem. \& Schult. 7. p. 7, 8. Brown-headed BeakSedge.

Ribes, L. Saxifrag'en, Saxifrages. Pent. Mon.

alpi'num, L. 32. s. 4, 5. Tasteless Mountain Currant.

Grossula'ria, L. s. 4, 5. Wild Gooseberry.

v. U'va-crisp'a ${ }^{11}$ (L.) Smooth Gooseberry.

ni'grum, L. s. 4, 5. Black Currant.

petræ'um, $\mathrm{Sm} .=\mathrm{R}$. ru'brum, v. petræ'um.

ru'brum, L. s. 4, 5. Red Currant. Wild Currant.

r. sati'vum ${ }^{12}$ (Reichb.) Cultivated Red Currant.

v. petræ'um, ${ }^{13} \mathrm{Sm}$. Rock Currant.

v. spica'tum ${ }^{14}$ (Robs.) Acid Mountain Currant.

sati'vum, Reichb. = R. ru'brum, v. sati'vum.

spica'tum, Robs. = R. ru'brum, v. spica'tum.

sylvest're, Syme.

v. Smithia'num = R. ru'brum, v. petræ'um.

v. spica'tum $=$ R. ru'brum, v. spica'tum.

U'va-crisp'a, L. $=$ R. Grossula'ria, v. U'va-crisp'a.

Robertso'nia, Haworth.

Ge'um = Saxif'raga Ge'um, L.

hirsu'ta $=$ Saxif'raga birsu'ta, L.

stella'ris = Saxif'raga stella'ris, $\mathbf{L}$.

umbro'sa = Saxif'raga umbro'sa, L.

Rœme'ria, DC. Papa'vera'ces, Poppy-worts. Polyan. Mon.

lyyb'rida, ${ }^{15}$ DC. a. 5, 6. Violet Horn-Poppy. Erect-podded Remeria.

Romul'ea, Maratti. InID'es, Irids. Tri. Mon.

Colum'næ, ${ }^{18}$ Seb. \& Maur. 1. p. 3-5. Columna's Trichonema. Ixia. ${ }^{17}$
L.C.

155

154

156

E. B.

i. 82

i. 81

B.

112

115

157

158

156

316

316

317

(ii.163)

ii. 162

114

113

ii. 163

ii. 162

ii. 318

ii. 318

223

2.24

1078 (vi.998)

767

1079

vi.999

$1079 \mathrm{a}$

$1079 \mathrm{~b}$

$1079 \mathrm{c}$

vi. 998

1548

X.1582

1092

1547

x.1581

1081

557

iv. 519

371

556

iv.518

369

$556 \mathrm{~b}$

iv. 523

372

558 iv. $520-2$

370

558a (iv.520)

$558 \mathrm{~b}$ (iv.521)

$558 \mathrm{c}($ iv. 522$)$

iv. 520

iv. 521

iv. 522

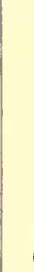

61

i. 64

43

1377 (ix.1492)

1 Jointed or White Charlock. 2 Reseda fruticulosa,-R. suffruticulosa. 3 Shrubby Base Rocket. 4 Base Dyer's Rocket.-Rocket Yellow-weed. 5 Wild Woad. 6 Alder or Breaking Buckthorn. 7 Rhinanthus minor. $8 \mathrm{R}$, augustifolius. 9 Schøens albus. io S. fuscus. 11 Ribes Ura-crispa. 12 R. sativum. 13 R. petraum. - lR. sylrestre, v. Smithiaum. 14 R. spicatum.-R. sylvestre, v. spicatum. 15 Chelidonium hybridum. - Glaucium violareum.

T. Bulbocodium. 17 Channelled Trichorema. 
Ros'a, Tourn. Rosa'cese, Roseworts. Icos. Pol.

[agrest'is, ${ }^{1}$ Savi.] 9. Small-leaved Swcetbriar.

v. Billiet'ii (Puget)

v. cryptu'pod'a, ${ }^{2}$ Baker.

v. in'odo'ra ${ }^{3}$ (Fr.)

arvens'is, Huds, 70, s. 6,7. White-flowered Trailing Dog-Rose. Field $R$.

จ. Briggs'ii (Gaud.)

v. bibract'ea'ta ${ }^{4}$ (Bast.)

Bake'ri. Deségl. = R. cani'na, v. Bake'ri.

bibract'ea'ta, Bast. $=$ R. arvens'is, v. bibract'ea'ta.

Borre'ri, Woods $=$ R. cani'na, v. Borre'ri.

bractesc'ens, Woods $=R$. cani'na, v. cor'iifol'ia.

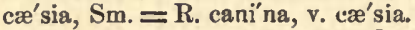

cani'na, L. 110. s. 6-8. Dog-Rose.

v. lutet'ia'na (Leman)

v. surculo'sa (Woods)

v. sphæ'rica (Gren.)

v. sentico'sa (Ach.)

v. duma'lis ${ }^{5}$ (Bechst.)

v. biserra'ta (Mérat.)

v. urb'ica ${ }^{6}$ (Leman)

v. frondo'sa (Steven.)

v. arva'tica, Baker.

v. dumeto' rum ${ }^{7}$ (Thuill.)

v. obtu'sifol'ja (Desv.)

v. pruino'sa, Baker.

v. inca'na (Woods)

v. to'mentell'a (Leman)

จ. and'egavens'is (Bast.)

v. vert'icill'acanth'a (Mérat.) .

v. colli'na (Jacq.)

v. Koscinc'ia'na (Besser.)

v. $\mathfrak{c æ}^{\prime} \operatorname{sia}^{8}(\mathrm{Sm}$.)

v. concinn'a, Baker.

v. decip'iens (Dum.)

v. glauc'a (Vill.)

v. subcrista'ta, Baker.

v. Hailsto'ni, Baker.

v. implex'a (Gren.)

v. cor'ifol'ia $^{9}$ (Fr.)

v. Watso'ni, Baker.

v. celera'ta, Baker.

v. Groves'ii, Baker.

v. Borre'ri ${ }^{10}$ (Woods)

v. Bake'ri ${ }^{11}$ (Déségl.)

v. margina'ta (Wallr.)

cinnamo'mea, L. s. 5-7. Cinnamon Rose.

colli'na, Sm. = R. stylo'sa, v. systy'la.

cor'iifol'ia, Fr. = R. cani'na, v. cor'iifol'ia.

crypt'opod'a, Baker $=$ R. agrest'is, v. crypt'opod'a.

Dickso'ni, Lindl. (or Dickso'nia'na) s. 6. Dickson's Rose.

Do'nia'na, Woods $=R$. involu'ta, v. Do'nia'na.

du'meto'rum, Sm. = R. cani'na, v. Borre'ri.

du'meto'rum, Thuill. = R. cani'na, v. du'meto'rum.

Forste'ri, Sm. $=$ R. cani'na, v. urb'ica.

gall'ica, L. s. 6. French Rose. 
Ros'a, Tourn.-continued.

hibern'jea, Sm. 8. s. 6, 7. Irish Burnet Rose.

v. gla'bra, Baker.

v. cordifol'ia, Baker.

in'odo'ra, Fr. $=R$. agrest'is, v. in'odo'ra.

involu'ta, Sm. 49. s. 6, 7. Prickly Unexpanded Rose.

v. Smith'ii, Baker.

v. Sabi'ni (Woods)

v. Dónia'na (Woods)

v. grac'ilis (Woors)

v. gracilese'ens, Baker.

v. Robertso'ni, Baker.

v. Nicholso'ni, Crépin.

v. læviga'ta, Baker.

v. Moore'i, Baker.

v. oc'cidenta'lis, Baker.

v. Wilso'ni ${ }^{3}$ (Borr.)

Jundzill'ia'na, Besser. s. Jundzil's Rose.

lu'cida, Ehrh. s. 7. Shining-leaved Rose.

mīeranth'a (Sm.) 54. s. 6, 7. Small-flowered Sweetbriar.

v. Briggs'ii, Baker.

v. hy'strix (Leman)

moll'is, ${ }^{4}$ Sm. 57. s. 6, 7. Soft-leaved Round-fruited Rose. ${ }^{5}$

v. cærul'ea, Woods.

v. pseud'o-rubi'gino'sa (Lej.)

molliss'ima, Fr. $=$ R. moll'is, Sm.

pimp'inell'ifol'ia, L. = R. spinosiss'ima, L.

pomif'era, Herrm.

rubell'a, Sm. s. 5: Red-fruited Burnet or Dwarf Rose.

rubi'gino'sa, L. 57. s. 6, 7. True Swcetbriar. Eglantine.

v. permix'ta (Déségl.)

v. sylvic'ola (Déségl. et Rip.)

Sabi'ni, Woods = R. involu'ta, v. Sabi'ni.

sarmenta'cea, Wouds $=$ R. cani'na, v. duma'lis.

sca'briusc'ula, $\mathrm{Sm} .=\bar{R}$. to'mento'sa, v. sca'briusc'ula.

semp'ervir'ens, L. s. 6. Evergreen Rose.

se'pium, Thuill. = R. agrest'is, Savi.

spinosiss'ima, ${ }^{6}$ L. 90. 5. 5, 6. Common Burnet Rose. ${ }^{7}$

v. Ripart'ii, Déségl.

stylo'sa, Desv. 37.

v. systy $1 a^{8}$ (Bast.)

v. leucoch'roa (Desv.)

v. opa'ca, Baker.

v. virgin'ca (Rip.)

จ. gall'jcoï'des, Baker.

v. Monso'nix, Lindl.

sylvest'ris, Lindl. $=\mathrm{R}$. to'mento'sa, $\mathrm{v}$. sylvest'ris.

systy'la, Woods = R. stylo'sa, r. systy'la.

to'mento'sa, Sm. 106. s. 6, 7. Downy-leaved Dog-Rose.

v. sub'globo'sa ( $\mathrm{Nm}$.)

v. farino'sa (Rau)

v. sca'briusc'ula ${ }^{\circ}(\mathrm{Sm}$.)

v. foétida (Bast.)

v. sylvest'ris ${ }^{10}$ (Lindl.)

v. Woodsia'na, Groves.

v. ob'ova'ta, Baker.

urb'ica, Leman $=$ R. cani'na, v. urb'ica.

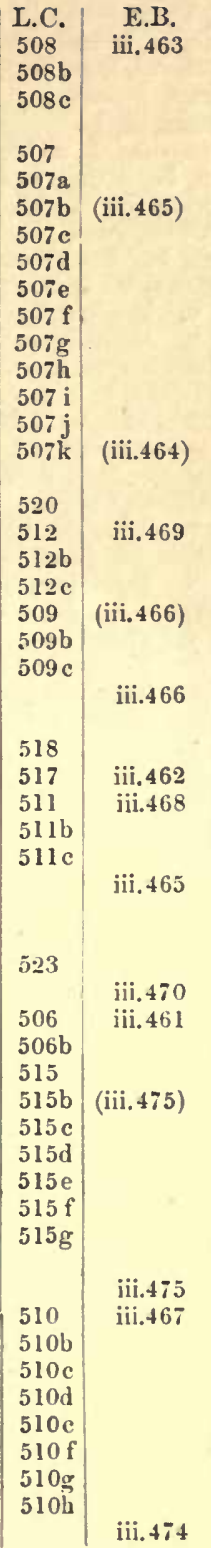

B.

327

329

1 Rosa Sabini. 2 R. Doniana. 3 R. Wilsoni. Apple Kose,-Douny Rase. 6 R. pimpinellifolia. 0 R. scabringcula. 10 R. sylvestris.
4 R. mollissima. - R, villosn. 5 Fillous Rose, 7 Scotch Rose. $\quad 8$ R. collina,-R. systyla. 
Ros'a, Tourn.-continued.

villo'sa, $\mathrm{L}$. $=\mathrm{R}$. moll' is, Sm.

Wilso'ni, Borr. = R. involu'ta, v. Wilso'ni.

RosA'CE. Roseworts.

Rottboell'ia, L.

incurva'ta, L. = Leptu'rus fi'liform'is, Trin.

Rub'ia, L. RuB'ia'cer, Stellates. Tetran. Mon. peregri'na, L. 23. p. 6-8. Wild Madder.

RUB'IA'CEE, Juss. Stellates.

Rub'us, L. Rosa'CEA, Roseworts. Icos. Pol.

affi'nis, W. \& N. 34. s. 7-9. Intermediate Bramble.

v. lenti'gino'sus (Lees)

v. cordifol'ius (W. \& N.)

althæ'ifol'ius, Host. $=$ R. deltö'deus, Müll.

aret'icus, L. p. 6. Arctic Bramble.

argent'eus, IV. \& N. s. 7, 8. Silvery Bramble.

Babingto'nii, Salt. 12. s. $7,8$.

Balfouria'nus, Blox. 18. s. 7, 8. Balfour's Bramble.

Bellard'i, Weihe. 9. s. 7, 8. Glandular-stemmed Bramble. v. denta'tus, Blox.

Bloxa'mii, Lees. 9. s. 7, 8. Bloxam's Bramble.

v. thyrs'iger, Bab.

cæ'sius, L. 68. s. 6, 7. Dewberry. Blue Bramble.

v. umbro'sus.

v. ten'uis (Bell-Salt.)

v. ligeri'nus (Genev.)

v. intermed'jus, Bab.

v. pseud'o-Idæ'us (Lej.)

v. hisp'idus, Bab.

carpi'nifol'ius, W. \& N. 25. s. 7, 8. Hornbeam-leaved $B$. certiflo'rus, P. J. Müll. 2.

Chamæmo'rus, L. 35. p. 6. Cloud-berry. Roebuck-berry. ${ }^{2}$

Colemann'i, Blox. 5. s. 7, 8. Coleman's Bramble.

cor'ylifol'ius, Sm. 38. s. 6-8. Hazel-leaved Bramble.

v. sublust'ris (Lees.)

v. conjung'ens, Bab.

v. fasc'icula'tus (P. J. Müll.)

de'bilis, Boul. ? 1.

deltoï'deus, ${ }^{3}$ P. J. Müll. 15. s. 6-8. Mallow-lecved Bramble. dis'color, W. \& N. $=$ R. rustica'nus, Merc.

divers'ifol'ius, Lindl. 25. s, 7, 8. Various-leaved Bramble. v. asperr'imus (Rip.)

dumeto'rum, Weihe. s. 7,8 .

cch'inatus, Lindl. 22. s. 7, 8.

emersisty'lus, P. J. Müll. 3. s. 7, 8.

erubesc'ens, Wirtg. 1.

fiss'us, Lindl. 24. s. 6-8. Lesser Sub-crect Bramble.

flexuo'sus, M. \& L. 10.

folio'sus, Weihe. 3. s. 7, 8. Leafy-flowered Bramble.

frutico'sus, L. s. 7-9. Common Bramble. Blackberry.

fusc'o-a'ter, Weihe (?) 4. s. 7, 8. Brownish-black Bramble.

Günthe'ri, ${ }^{4}$ Weike. 10. s. 7,8. Günther's Bramble.

he'mistémön, P. J. Müll. 6. s. `, 8.

hirtifol'ius, Muill. 2. s. 7, 8 .

hirt'us, W. \& N. 12. t. 8.

v. rotund'iful'ius (Blox.)

hǔmifu'sus, Weihe. 12, s. 7, 8. Trailing Bramule. 
Rub'us, I. - continued.

Hy'strix, Weihe. = R. rosa'ceus, v. Hy'strix.

Idæ'us, L. 105. s. 6. Common Raspberry.

v. Lees'ii ${ }^{1}$ (Bab.) Lees's Raspberry.

v. rotund'ifol'ius, Bab.

imbrica'tus, Hort. 4. s. 6, 7. Imbricated-leaved Bramble.

incurva'tus, Bab. 10. s. 7. Incurved-leaved Bramble.

Toehle'ri, Weihe. 34. s. 7, 8. Köhler's Bramble.

v. infest'us, Bab.

v. egreg'ius (Focke)

v. Schlicku'mi (Wirtg.)

v. pall'idus, ${ }^{2}$ Bab.

v. cava'tifol'ius (P. J. Müll.)

la'tifol'ius, Bab. 3. s. 7, 8. Broad-leaved Bramble.

Lees'ii, Bab. = R. Idx'us, v. Lees'ii.

Lejeun'ei, Weihe. 15. s. 7, 8. Lejeune's Bramble. v. festi'vus (M. \& W.)

leuc'ostach'y̌s, Sm; 38. s. 7, 8. Long-clustered or Downy-spiked B. v. conspic'uus'(P. J. Müll.)

Lind'leia'nus, Lees. 39. s. 7, 8. Lindley's Bramble.

long'ithyrs'iger, Lees. 9.

Maass'ii, Focke. 34.

mācrophyll'us, Wr. \& N. 28. s. 7, 8. Large-leaved Bramble.

v. Schlec'tenda'lii (W. \& N, )

v. am'plifica'tus (Lees.)

v. glabra'tus, Bab.

monta'nus, Wirtg. 5 .

mu'crona'tus, ${ }^{3}$ Blox. 18. s. 7, 8. Cuspidate-leaved Bramble. mucro'nula'tus, Boreau = R. mu'crona'tus, Blox.

muta'bilis, Genev. 1. s. 7,8.

nem'oro'sus, Hayne. s. 6, 7. Larger Dewberry.

nit'idus, W. \& N. 3.

v. ha'mulo'sus, P. J. Müll.

pall'idus, Weihe $=\mathrm{R}$. Koehle'ri, v. pall'idus.

pampino'sus, Bab. $=\mathrm{R}$. villicau'lis, Ḱoehl.

penduli'nus, P. J. Miill. 1.

pubesc'ens, W. \& N. 16.

pygmæ'us, Weihe. $=R$. prærupto'rum, Boul.

py'ramida'lis, Kalt. 5. s. 7, 8. Pyramidal-flowered Bramble.

Ra'dula, Weihe. 28. s. 7, 8. File-stemmed Bramble.

v. Leighto'nii (Lees)

ramo'sus, Blox. 3.

Reute'ri, Mere. 4.

rhamnifol'ius, W. \& N. 32. s. 7, 8. Buckthorn-leaved Bramble.

rosa'cens, W. \& N. 21. s. 7, 8. Rose-flowered Bramble.

v. Hy'strix, Weihe. Hedgehog Bramble.

rub'eolus, Weibe $=R$. salte'ri, Bab.

rud'is, Weihe. 1. s. 7, 8.

rustica'nus, ${ }^{8}$ Merc. 43 . s. 7, 8. Common Bramble.

Salte'ri, ${ }^{7}$ Bab. 14. s. 7, 8. Salter's Bramble.

v. calva'tus, Blox.

salt'uum, Focke $=$ R. Giinthe'ri, Weihe.

saxa'tilis, L. 61. p. 7, 8. Stone Bramble. Roebuck-berry.

saxic'olus, P. J. Mitll. 12.

v. sphe'noï'deus (Boul.)

scab'er, W. \& N. 3. s. 7, 8. Rough Bramble.
L.C.

416

$416 \mathrm{~b}$

$416 \mathrm{c}$

427 .

426

456

$456 b$

$456 \mathrm{c}$

$456 \mathrm{~d}$

$456 \mathrm{e}$

$456 \mathrm{f}$

429

461

$46 \mathrm{lb}$

433

$433 b$

424

462

440

441

$44^{\prime} \mathrm{lb}$

$441 \mathrm{c}$

44ld

435

442

460

421

$421 b$

467

$+19$

448

431

E.B.

B.

iii. 442

(iii.443)

iii. 453

iii. 443

iii. 448

iii. 450

(iii.451)

iii. 451

iii. 445

434

455

$455 \mathrm{~b}$

428

469

425

447

$447 b$

453

432

437

437b

475

464

$464 \mathrm{~b}$

450 
Rub'us, L.-contimued.

scabro'sus, ${ }^{1}$ P. J. Muill. 16. s. 7, 8. Tubercular Bramble. v. trigo'nodont'us (Boul.)

Sprenge'lii, Weihe. 16. s. 6, 7. Sprengel's Bramble. v. Borre'ri (Bell-Salt.)

suberect'us, $A$ nders. 30. s. 6, 7. Sub-erect B. Red-fruited $B$. sulca'tus, Vest. 1.

thyrsiflo'rus, W. \& N. 4.

v. phil'yrophyll'us (L. \& M.)

thyrsoi'deus, Wimm. 1. s. 7, 8. Thyrsus-flowered Bramble. tuberc'ula'tus, Bab. $=$ R. scabro'sus, Müll.

umbro'sus, Arrb.

villican'lis, ${ }^{2}$ Koehl. 28. s. 7, 8. Pilose-stemmed Bramble.

v. adsci'tus, Genev.

จ. gra'tus, Focke.

vulga'ris, Lindl. A form of R. villicaul'is, Koehl.

Wahlberg'ii, Arrh. A form of R. cor'ylifol'ius, Sm.

Rúmex,' I. Por'rgona'ce.e, Buckwheats. Hex. Trig.

Aceto'sa, L. 112. p. 5, 6. Common Sorrel.

Ace'tosell'a, L. 112. p. 5-7. Sheep's Sorrel. acu'tus, ${ }^{3}$ L. 55. p. 6-9. Meadow Dock.

alpi'nus, L. p. 7. Monk's Rhubarb. Alpine Dock.

aqua'ticus, ${ }^{4}$ L. 37. p. 7, 8. Grainless Curled Water Dock.

con'glomera'tus, ${ }^{3}$ Murr. 92. p. 6-8. Sharp Dock.

v. rupest'ris ${ }^{6}$ (Le Gall) 6. Rock Dock.

conspers'us, Hartm. 9. p. 6-8. Hartman's Dock.

crisp'us, L. 109. p. 6-8. Curled Dock.

v. trigra'nula'tus, Syme.

v. sub'corda'tus, Warren.

v. elonga'tus ${ }^{7}$ (Guss.)

digyn'us, L. = Oxyr'ia digyn'a, Hill.

domest'icus, Hartm. $=$ R. aqua'ticus, $L$.

elonga'tus, Guss. $=$ R. crisp'us, v. elonga'tus.

glom'era'tus, Spr. = R. con'glomera'tus, Murr. hyb'ridi, " teste Trimen."

a. pulch'er $\times$ con'glomera'tus.

b. pulch'er $\times$ obtu'sifol'ius.

c. pulch'er $\times$ rupest' ris.

d. pulch'er $\times$ crisp'us.

e. crisp'us $\times$ nem'oro'sus.

f. con'glomera'tus $\times$ marit'imus.

g. con'glomera'tus $\times$ sylvest' $r$ is.

Hy'drolap'athum, Huds. 68. p. 7, 8. Great Water Dock.

v. Ja'tifol'ia, ${ }^{8}$ Borr. 6.

limo'sus, Thnill. See palust'ris, Sm.

marit'imus, L. 38. p. or b. 7, 8. Golden Dock.

max'imns, Schreb. = R. Hy'drolap'athum, v. la'tifol'ia.

nem'oro'sus, Schrad. $=R$. sanguin'eus, v. vir'idis.

Nem'olap'athum, Wallr. = sanguin'eus, v. vir'idis.

obtu'sifolius, L. 109. p. 7-9. Broad-leaved Dock.

v. sylvest'ris (Wallr.) 5 .

1 Rubus tuberculatus. 2 R. pampinosus. 3 Rumex pratensis. 4 R. domesticus. 5 R. glomeratus. R. rupestris. 7 R. elongatus. 8 R. maximus. 9 R. nemorosus, $-R$. Nemolapathum. 
Rupp'ia, L. NáIAdA'CEs, Naiads. Tetran. Tetrag. marit'ima, L.

rostell'ata, Koch. 28. p. 7, 8. Lesser Ruppia.

v. na'na, Bosw. v. na'na, Bosw.
spira'lis, Hartm. 15. p. 7, 8. Greater Ruppia. Sea Tassel-
grass.

Rusc'us, L. Lr'LIA'CEE, Lilyworts. Diœc. Trian. acu'lea'tus, L. 29. s. 2-4. Butcher's Broom.

L.C.

Sagi'na, L. CAR'YopmrL'E. E, Cloveworts. Tetran. Tetrag. apet'ala, L. 56. a. 5-8. Common Small-flowered Pearlwort. v. prostra'ta, Bab.

cil'ia'ta, Fr. 61. a. 5, 6. Fries' Small-flowered, or Ciliated P. cerast'oídes, $\mathbf{S m}$. = Cerast'ium tetrand'rum, Curt.

dens'a, Jord. = S. marit'ima, $\mathbf{v}$. dens'a.

erect'a, L. = Cerast'ium quāternell'um, Fenzl.

Linnæ' $1,{ }^{2}$ Presl. 7. p. 6-8. Alpine Pearlwort.

marit'ima, Don. 62. a. 5-9. Sea Pearlwort.

v. de'bilis (Jord.)

v. dens' $\mathrm{a}^{3}$ (Jord.)

v. alpi'na, Syme.

niva'lis, ${ }^{4}$ Fr. 2. p. 6-8. Lindblon's Pearlwort.

nodo'sa, ${ }^{5}$ E. Mey. 95. p. 7, 8. Knotted Spurrey. Sand Chickwced. procumb'ens, L. 112. p. 5-9. Procumbent Pearlwort.

v. spino'sa, S. Gibs.

saxa'tilis, Wimm. =S. Linnæ'i, Presl.

su'bula'ta, ${ }^{6}$ Presl. 59. p. 6-8. Awl-shaped Pearlwort.

Sag'itta'ria, L. Al'rsma'ces, Alismads. Monœe. Pol. sagitt'ifol'ia, L. 57. p. 7-9. Common Arrow-head.

SAL'ICIN'E. Willowworts.

Salicorn'ia, Tourn. Che'ropon'ríce. Chenopods. Monan. Mon. ann'ua, Sm. = S. herba'cea, $\mathrm{L}$.

frutico'sa, Sm. = S. radi'cans, Sm.

herba'cea, ${ }^{7}$ L. 64. a. 8,9. Common Marsh Samphire. ${ }^{8}$

v. aceta'ria, Moq-Tand.

r. procumb'ens ${ }^{\mathrm{g}}(\mathrm{Sm}$.)

v. ramosiss'ima, Woods.

v. pusill'a, Woods.

procumb'ens, $\mathrm{Sm} .=\mathrm{S}$. herba'cea, v. procumb'ens.

radi'cans, ${ }^{10} \mathrm{Sm}$. 12. s. 9, 10 . Creeping Marsh Samphire. ${ }^{11}$

Sal'ix, Tourn. Sal'icrN'Ee, Willowworts. Diœc. Dian.

acu'mina'ta, Sm. t. or s. 4. Long-leaved Sallow.

acu'tifol'ia, Willd. $=$ S. daphnoi'des, Vill.

alb'a, L. 86. t. 5. Common White Willow.

v. crerul'ea ${ }^{12}(\mathrm{Sm}$.$) Blue Willow.$

v. vitelli'na ${ }^{13}$ (L.) Golden Willow or Osier.

anıig'ua, Ehrb. 23. s. 4. Ambiguous Sallow or Willow.

r. genui'na, auct.

v. ma'jor, Syme.

v. spăthula'ta (Willd.)

v. undula'ta, Syme.

amygd'ali'na, L. = S. triand'ra, v. amygd'ali'na.

Anderso'nia'na, Sm. = S. ni'gricans, v. Anderso'nia'na. angust'ifol'ia, Wulf. =S. ro'smari'nifol'ia, v. angust'ifol'ia. aqua'tica, $\mathrm{Sm} .=\mathrm{S}$. ciner'ea, v. aqua'tica.
1509

$1509 \mathrm{~b}$

1508

1389

\begin{tabular}{l|l} 
E. B. & B. \\
ix. 1427,8 & 952 \\
ix. 1428 &
\end{tabular}

ix. 1427

ix.1516

1031

240

$240 \mathrm{~b}$

ii. 246

241

ii. 247

243

(ii.249)

239

$239 \mathrm{~b}$

$239 \mathrm{c}$

$239 \alpha$

244

246

242

$242 b$

245

ii. $250 \mathrm{bis}$

ii. 251

ii. 248

147

145

ii. 249

ii. 250

1473 ix. 1436

965

1280

1200

viii. 1181,2

836

viii. 1181

$1200 \mathrm{~b}$ viii. 118.2

$1200 \mathrm{c}$

$1200 \mathrm{~d}$

1201 viii. 1183

1294 viii. 1326

viii. 1366

1282 viii. 1309

1282 b viii. 1310

$1282 \mathrm{c}$ viii. 1311

1301 viii. 1355

$1301 \mathrm{a}$

$1301 \mathrm{~b}$

$1301 \mathrm{c}$

$1301 \mathrm{~d}$

918
1 Tassel Pondweed. nivslis. $\quad 3$ S. nodoss. procumbens. 13 $\mathrm{S}$, vitellins.
2 Sagina saxatilis.-Spergula saginoides. 6 S. subulata. 10 \$. fruticosa. 7 Balicornia annus. 8 Jointed Glass
3 Sagina densa.

4 Spergula 9 Salicornis 12 Salix crernles. 
Sal'ix,-contimued.

Arbusc'ula, L. 7. s. 6,7. Plum-leaved Willow. Small Tree W.

v. carina'ta' (Sm.) Folded-leaved Willow.

v. fœ'tida (Schl.)

v. ve'nulo'sa ${ }^{2}$ (Sm.) Veiny-leaved Willow.

v. racci'niifol'ia ${ }^{3}$ (Walker) Bilberry-leaved Willow.

v. pru'nifol'ia, ${ }^{4} \mathrm{Sm}$.

ar'ēna'ria, $\mathrm{L}_{\text {. }}=\mathrm{S}$. lappo'num, v. ar'ēna'ria.

$\operatorname{argent}$ ea, $\mathrm{L} .=\mathrm{S}$. re'pens, $\mathbf{v}$. argent'ea.

ascend'ens, $\mathrm{Sm} .=\mathrm{S}$. re'pens, v. ascend'ens.

auri'ta, L. 99. s. 4, 5. Wrinkled-leaved or Round-eared Sallow.

v. min'or, Sonder.

bicol'or, Sm. = S. lauri'na, Sm.

Borre'ria'na, Sm. = S. phyl'iciful'ia, v. Borre'ria'na.

Cap'rea, L. 89. t. or s. 4, 5. Great Round-leaved S. Goat Willow.

v. sphăcela'ta ${ }^{5}$ (Sm.) Withored-pointed Sallow.

carina'ta, $\mathrm{Sm} .=\mathrm{S}$. Arbusc'ula, v. carina'ta.

cærul'ca, Sm. $=$ S. alb'a, v. cærul'ea.

ciner'ea, L. 102. t. or s. 4. Common or Gray Sallow.

v. aqua'tica ${ }^{6}(\mathrm{Sm}$.) Water Sallow.

v. ol'ếfol'ia ${ }^{7}(\mathrm{Sm}$.) Olive-leaved Sallow.

coto'nifol'ia, Sm. = S. ni'gricans, v. coto'nifol'ia.

Crowea'na, Sm. = S. phyl'icifol'ia, v. Crowea'na.

cuspida'ta, Schultz. t. 6, 7. Pointed.leaved W. Cuspidate $W$. damasce'na, Forbes $=\mathrm{S}$. ni'gricans, $\mathbf{v}$. damasce'na.

daphnoi'des, ${ }^{8}$ Vill. t. 4. Violet Willow.

Davall'ia'na, Sm. = S. phyl'icifol'ia, v. Davall'ia'na.

decip'iens, Hoffm. $=\mathrm{S}$. frag'ilis, $\mathbf{v}$. decip'iens.

Dickso'nia'na, Sm. = S. pbyl'icifol'ia, v. Dickso'nia'na.

Do'nia'na, Sm. s. 5. Donian W. Rusty-branched Willow.

ferrugin'ea, G. Anders. s. 4. Ferruginous Osier.

fœétida, $\mathrm{Sm} .=\mathrm{S}$. re'pens, vv. ascend'ens \& parvifol'ia.

Forbya'na, Sm. $=$ S. ru'bra, v. Forbya'na.

Forste'ria'na, Sm. = S. ni'gricans, v. Forste'ria'na.

frag'ilis, I. 84. t. 4, 5. Crack Willow. Withy.

v. decip'iens, ${ }^{9}$ Hoffm. White Welsh or Varnished Willow.

v. Russellia'na ${ }^{10}(\mathrm{Sm}$.)

fusc'a, L. = S. re'pens, v. fusc'a.

glauc'a, Sm. = S. lappo'num, v. pseud'o-glauc'a.

Graba'mi, Borr. 1. s. 6, 7. Graham's Willow.

v. Bake'ri, Lond. Cat. 1.

v. Moore'i, Lond. Cat. I.

hasta'ta, ${ }^{11}$ L. s. 5. Apple-leaved Willow.

$\mathrm{Hel}^{\prime} \mathrm{ix}, \mathrm{Sm}$. = S. ru'bra, v. Hel'ix.

herba'cea, L. 25. s. 6. Dwarf or Least Willow.

hirt'a, Sm. = S. ni'gricans, v. hirt'a.

Hoffmannia'na, Sm. = S. triand'ra, r. Hoffmanuia'na.

bol'oseric'ea, Willd. s. 4, 5. Soft Shaggy-flowered Willow.

incuba'cea, $\mathrm{L}$. $=\mathrm{S}$. re'pens, $\vee$. incuba'cea.

Lambert'ia'na, Sm. = S. purpur'ea, v. Lambert'ia'na.

lana'ta, L. 3. s. 5, 6. Woolly Broad-leaved Willow.

lanceola'ta, Sm. $=$ S. undula'ta, Ebrb.

lappo'num, L. 13. s. 6, 7. Downy Mountain Willow.

v. $\operatorname{ar}^{\prime}$ ena'ria $^{12}$ (L. ex p.)

v. Stuartia'na ${ }^{13}(\mathrm{Sm}$.$) Small-leaved Shaggy W$.

v. pseud'o-glauc'a, ${ }^{14}$ Syme. Glaucons Mountain $W$.

lauri'na, ${ }^{15} \mathrm{Sm}$. 11 . t. or s. 4,5 . Intermediate Sallow. ${ }^{16}$

L.C. E.B.

B.

1305 viii. $1371-4$

1305 a viii. 1371

$1305 \mathrm{~b}$

$1305 \mathrm{c}$ viii. 1373

1305 d viii. 1374

viii. 1372

1296

viii. 1330

922

$1296 \mathrm{~b}$

1297 riii. 1331

921

$129 \%$ b viii. 1332

1295 viii. 1327

$1295 \mathrm{~b}$ viii. 1328

1295 c viii. 1329

1280

viii. 1304,5

1288 (viii.1366)

1286 Tiii.136.5

1292 viii. 1325

1281 viii. 1306

916

1281 b viii. 1307

$1281 \mathrm{c}$

$1281 c$

1308. viii. 1377

1308 a

$130 \mathrm{sb}$

1309 viii. 1378

929

1303

viii. 1367

926

1304 viii. $1368-70$

925

1 Salix carinata. 2 8. renulosa. $3 \mathrm{~S}$. livida, Sm. $-\mathrm{S}$. vaccinilfolia. 4 S. prunifolia. 5 s. sphacelata. 6 8. aquatica. $7 \mathrm{~S}$. oleifolia. $8 \mathrm{~S}$. acutifolia. 9 S. decipiens. io S. Russellians. 11 S. malifulia. 12 S. arenaria. $13 \mathrm{~S}$. limasa.-S. Stuartiana. $14 \mathrm{~S}$. glanca. $15 \mathrm{~g}$. bicolnr, Sm. 16 Laurel-leaved Willow.-Shining Dark-green $W$. 
Sal'ix, Tourn.-continued.

laxiflo'ra, Borr. = S. phyl'icifol'ia, v, laxifo'ra.

limo'sa, Wahl. = S. lappo'num, v. Stuartia'na.

li'vida, Sm. = S. arbusc'ula, v. vacci'niifol'ia.

li'vida, Wabl. = S. phyllicifol'ia, v. Weige'lia'na. málifol'ia, Sm. = S. hasta'ta, L.

molliss'ima, Sm. =S. Smithia'na, Willd.

Myrsini'tes, L. 7. s. 6, 7. Green Whortleberry-leaved Willow.

v. serra'ta, Syme.

v. procumb'ens ' (Forbes) Smooth-leaved Alpine $W$.

v. arb'utifol'ia, Syme.

ni'gricans, "Sm." 19. s. 5, 6. Dark Broad-leaved Sallow.

v. genui'na, auct. Quince-leaved $S$. Sumach-leaved W.

v. coto'nifol'ia ${ }^{2}(\mathrm{Sm}$.

v. Forste'ria'na ${ }^{3}$ (Sm.) Glaucous Mountain Sallow.

v. rupest'ris ${ }^{+}$(Sm.) Silky Rock Willow.

v. Anderso'nia'na ${ }^{5}$ (Sm.) Green Mountain Sallow.

v. damasce'na ${ }^{6}$ (Forbes) Damson-leaved Willow.

v. petra'a (G. Anders.) Dark Rock Willow.

v. hirt'a ${ }^{7}$ (Sm.) Hairy-branched Willow.

v. Ho'ribund'a ${ }^{3}$ (Forbes)

nit'ens, And. = S. phyl'icifol'ia, v. nit'ens.

ol'ěfol'ia, Sm. = S. ciner'ea, v. ol'ěfol'ia.

parvifol'ia, Sm. = S. re'pens, v. parvifol'ia.

pentand'ra, L. 54. s. or t. 5, 6. Sweet Bay-leaved Willow.

pet'iola'ris, Sm. t. 4. Dark Long-leaved Willow.

phillyr'exfol'ia, Borr. = S. phyl'icifol'ia, v, phillyr'exfol'ia.

phyl'icifol'ia, "Linn." 24. s. 4,5. Tea-leaved Sallow or Willow.

v. radi'cans $^{9}(\mathrm{Sm}$.)

v. Davall'ia'na ${ }^{10}(\mathrm{Sm}$.$) Darallian Willow.$

v. Weige'lia'na ${ }^{11}$ (Willd.) Wulfenian Willow.

v. nit'ens ${ }^{12}$ (G. Anders.) Shining.leaved Willow.

v. Crowea'na ${ }^{13}$ (Sm.) Broad-leaved Monadelphons Willous.

v. Dickso'nia'na ${ }^{14}$ (Sm.) Broad-leaved Mountain Willow.

v. ten'uior $^{15}$ (Borr.)

v. laxiflo' ${ }^{16}{ }^{16}$ (G. Anders.) Loose-flowered Willou.

v. proping'ua ${ }^{17}$ (Borr.)

v. tetrap'la ${ }^{16}$ (Walker) Four-ranked Willow.

v. Borre'ria'na ${ }^{19}$ (Sm.) Dark Upright Willow.

v. phillyr'eæfol'ia ${ }^{20}$ (Borr.)

v. ten'uifol'ia ${ }^{21}$ (Borr.) Thin-leaved Willow.

procumb'ens, Forbes $=\mathrm{S}$. Myrsini'tes, v. procumbens.

proping'ua, Borr. = S. phyl'icifol'ia, v. propinq'ua.

prostra'ta, Sm. $=\mathrm{S}$. re pens, v. prostra ta.

pru'nifol'ia, $\mathrm{Sm} .=\mathrm{S}$. arbusc'ula, $\mathbf{}$. pru'nifol'ia.

purpur'ea, L. 70. s. 4, 5. Bitter Purple Willow or Osior.

v. Woolga'ria'na ${ }^{22}$ (Borr.)

v. ra'mulo'sa (Borr.)

v. Lambert'ia'na ${ }^{23}$ (Sm.) Boyton Willow.

radi'cans, $\mathrm{Sm} .=\mathrm{S}$. phyl'icifol'ia, v. radi'cans.

re'pens, L. 92. s. 4, 5. Dwarf or Creeping Willow.

v. genni'na, L.

v. fusc'a, ${ }^{24}$ (L.) Brownish Dwarf Silky Willow.

v. prostra'ta ${ }^{25}$ (Sm.) Early Prostrate Willow.

$\therefore$ ascend'ens ${ }^{23}$ (Sm.) Fishy Willow.

\begin{tabular}{|l|c|c} 
L.C. & E.B. & B \\
1306 & viii. 1375,6 & 9
\end{tabular}

1306 a viii. 1375

$1306 b$ viii. 1376

$1306 \mathrm{c}$

1300 riij.1347 54

1300 a viii. 1347

$1300 \mathrm{~b}$ viii. 1348

$1300^{\circ} \mathrm{c}$ viii. 1349

$1300 \mathrm{~d}$ viii. 1350

1300 e viii. 1351

1300 f viii. 1352

$1300 \mathrm{~g}$ viii.1353

1300 h viii. 1354

$1300 \mathrm{i}$ riii. 1351 bis

1250

riii. 1303

1299 rii. $1331-4$

1299 b viii. 1335

1299 c viii. 1336

1299 d viii. 1337

1299 e viii.1335

1.299 f viii.1339

$1299 \mathrm{~g}$ viii. 1340

1299 h viii. 1341

1299 i viii. 1342

1299 j viii. 1343

$1299 \mathrm{k}$ viii.134t

12991 viii.1345

$1299 \mathrm{~m}$ viii. 1346

1285 viii.1316้

1285 b viii. 1317

$1285 \mathrm{c}$

$1285 \mathrm{~d}$ viii. 1318

1302 viii.1356-63

1302a viii. 1356

1302 b viii. 1357

1302 c viii. 1358

1302d viii. 1359
915

923

919

B.

927

924

1 Salix procumbens.

2 \$. cutonifolia.

3 \$. Forsterians.

4. rupestris.

5 \$. Andersoniana. $6 \mathrm{~S}$. damascens, $7 \mathrm{~S}$. hirta. $8 \mathrm{~S}$. tenuifolia, Sm. $9 \mathrm{~S}$. radicans,

$15 \mathrm{8}$. tennior. $16 \mathrm{~g}$. laxiflora. $17 \mathrm{8}$. propingua.

:0 8. pbillyreatolia.

21 S. tenulfolia, Borr.

22 S. Woolgariana.

Croweana.

14 \$. Dicksoniana.

25 S. prostrala.

$26 \mathrm{9}$. ascendens, - S. foetida. $23 \mathrm{~S}$. Lambertiana.

19. Dicksoniana. 
Sal'ix, Tourn.-continued.

v. parvifol'ia ${ }^{1}(\mathrm{Sm}$.

v. incuba'cea, ${ }^{2}$ Syme. Trailing Silk Willow.

v. $\operatorname{argent}^{\prime} a^{3}$ (Sm.) Silky Sand Willow.

re'ticula'ta, L. 5. s. 7, 8. Wrinkle or Reticulate-leaved Willow. ro'smari'nifol'ia, "L." Koch. s. 4. Rosemary-leaved Willow.

v. angust'ifol'ia." Little Tree Willow.

ru'bra, Huds. 36. t. or s. 4, 5. Green-leaved Osier.

v. Forbya'na ${ }^{5}(\mathrm{Sm}$.$) 2. Fine Basket Osier.$

v. $\operatorname{Hel}^{\prime}{ }^{6}{ }^{6}$ (L.) Rose Willow.

rugo'sa, Leefe. s. $4,5$.

rupest'ris, $\mathrm{Sm} .=\mathrm{S}$. ni'gricans, v. rupest'ris.

Russcllia'na, Sm. $=$ S. frag'ilis, v. Russellia'na.

Sadle'ri, Syme. 1. s. 8. Sadler's Willow.

Smithia'na, Will. s. 4, 5. Silky-leaved Osier.

v. pseud'o-stipula'ris, Lond. Cat.

sphăcela'ta $(\mathrm{Sm})=.\mathrm{S}$. cap'rea, v. sphăcela ta.

stipula'ris, Sm. s. 4. Auricled Osier.

Stuartia'na, Sm. = S. lappo'num, v. Stuartia'na.

ten'uifol'ia, Borr. = S. phyl'icifol'ia, v. ten'uifol'ia.

ten'uifol'ia, Sm. E. B. = S. ni'gricans, v. flo'ribund'a.

ten'uior (Borr.) =S. phyl'icifol'ia, v. ten'uior.

tetrap'la, Sm. = S. phyl'icifol'ia, v. tetrap'la.

triand'ra, L. 63.t.ors. 4-6. Almond-leaved Willow. Smooth W.

v. amygd'ali'na ${ }^{9}$ (L.)

v. Hoffmannia'na ${ }^{10}(\mathrm{Sm}$.

v. Trevira'ni (spr.)

v. contort' $a$ (Crowe)

undula'ta, ${ }^{11}$ Ehrh. 12. t. 4. Sharp-stipuled Triandrous Willow. vacci'niifol'ia, $\mathbf{S m} .=\$$. Arbusc'ula, v. vacci'niifol'ia.

ve'nulo'sa, $\mathrm{Sm} .=\mathrm{S}$. Arbusc'ula, v. re'nulo'sa.

vi'mina'lis, L. 80. s. or t. 4-6. Common Osier.

$v$. intrica'ta, Leefe.

vir'idis, Fr. t. 6. Bedford Willow.

vitelli'na, $\mathrm{L}_{0}=\mathrm{S}$. alb'a, v. vitelli'na.

Weige'lia'na, Borr. = S. phyl'icifol'ia, v. Weige'lia'na.

Woolga'ria'na, Borr. = S. purpur'ea, v. Woolga'ria'na.

Wulfe'nia'na, Sm. = S. phyl'icifol'ia, v. Weige'lia'na.

Sals'ola, I. CHE' Nopon'ia'Cex, Chenopods. P'ent. Dig.

frutico'sa, L. = Suæd'a frutico'sa, Forsk.

Ka'li, L. 58, a. 7, 8. Prickly Saltwort.

Salv'ia, L. LAB'1A'т Le, Labiates. Dian. Mon.

cland'esti'na, L. ? = S. Verbena'ca, v. clandesti'na.

pratens'is, L. 3. p. 6-8. Meadow Clary or Sage.

Verbena'ca, L. 64. p. 6-9. Wild English Clary or Sage.

v. clandesti'na ${ }^{12}$ (L.) Small-flowered Clary or Sage.

Sambu'cus, L. Cap'rifol'ia'Cen, Caprifoils. Pent. 'T'rig.

I:b'ulus, L. 74. p. 7, 8. Danewort. Dwarf Elder.

ni'gra, L. 106. s. 6. Common Elder.

v. lac'inia'ta, L.

Sam'olus, Tourn. Príntráces, Primworts. Pent. Mon.

Val'erand'i, L. 80.p.6-9. Brookweed. Samole. Water Pimpernel.

1284

viii. 1313

1284 a viii. 1315

1284 b viii. 1314

$1284 \mathrm{c}$

$1284 \mathrm{~d}$

1283

1289

$1289 \mathrm{~b}$

viii.1322

viii. $130 s$

1204

viii. 1180

vii. 1057

1127

1126

vii. 1058

vii.1056

(vii.1057)

684

iv. 638

683

iv. 637

$683 \mathrm{~b}$

960

vii. 1151

iii. 421

614 iv.568
B.

928

920

839

774

775

460

459

660

324

396
1 Salix parvifolia. $-S$. fotida. $6 \mathrm{~S}$, Helix. amygdaliaa.
7 S. mollissima.

$10 \mathrm{~S}$. Hoffmanniana.
2 S. incubacea. $3 \mathrm{~S}$. argentea. 8 Blunt-stipuled or Long-leaved Triandrous Willow.

5 S. Forbyana. $11 \mathrm{~S}$. lanceolata. 12 Salvia clandestina. 
Santoli'na, L.

\section{Satyr'ium, L.}

Sartald'Ce.e, Br. Sandalworts.

marit'ima, $\mathrm{Sm} .=$ Dio'tis marit'ima, Cass.

SAPINDA'CEE, Juss. Soapworts.

Sa'pona'ria, L. Car'yofurld'es, Cloveworts. Dec. Dig. oftic'ina'lis, L.p. 7. Common Soapwort. Fuller's Herb. Bruisewort. v. puber'ula, Syme.

Vacca'ria, L. a. 7. Cow-herb. Soapwort.

Sarotham'nus, Wimm.

scopa'rius, Koch = Cyt'isus scopárius, Link.

alb'idum, L. = Hăbena'ria alb'ida, R.Br.

hirci'num, L. = Orch'is hirci'na, Scop.

re'pens, $\mathrm{L}$. $=$ Goodye'ra re'pens, R.Br.

vir'ide, L. = Hăbena'ria vir'idis, R.Br.

Saussu'rea, DC. Compos'1te, Composites. Syn, Eq. alpi'na, ${ }^{1}$ DC. 21. p. 8. Alpine Sawwort.

Saxif raga, L. SAxifrá'es, Saxifrages. Dec. Dig. affi'nis, Don. = S. sponhe'mica, Gmel.

aizoï'des, ${ }^{2}$ L. 32. p. 6, 7. Yellow Monntain Saxifrage.

Andrews'ii, Harrey. p. 7. Andrew's London Pride.

cespito'sa, L. 3. p. 5. Tufted Alpine Saxifrage. Palmate S. cern'ua, ${ }^{3}$ L. 1. 1. 7. Drooping Alpine or Bulbous Saxifrage.

$\mid \begin{array}{r}\mathrm{L} . \\ 1243 \\ 31 \\ \\ 19 \\ 19 \\ 19\end{array}$ decip'iens, Ehrh. 1. p. 5. Palmate-leaved Mossy Saxifrage. de'nuda'ta, Don. p.

elongell'a, Sm. $=$ S. hy'pnoi'des, v. plat'ypet'ala, E.B.

ger'anoï'des, L. p. 4. Geranium Saxifrage.

Ge'um, L. I. p. 6. Kidney-leaved London Pride. ${ }^{5}$

v. crena'ta, Syme.

v. denta'ta, Lond. Cat.

v. e'leguns, Mackay.

v. serra'ta, Syme.

gra'nula'ta, ${ }^{6}$ L. 75. p. 4, 5. White Meadow Saxifrage.

Hirc'ulus, ${ }^{7}$ L. 8. p. 8. Yellow Marsh Saxifrage.

hirsu'ta, ${ }^{8}$ L. I. p. 5. Hairy London Pride.

hirt'a, Don. $=$ S. Sternberg'ii, Willd.

v. incurv'ifol'ia. ${ }^{9}$

v. affi'nis = S. sponhe'mica, Gmel.

hy'pnoï'des, L. 46. p. 5-7. Mossy Saxifrage. Ladies' Cushion.

v. plat'ypet'ala, ${ }^{10}$ Syme. Broad-flowered Saxifrage.

v. gemmif'era, Syme.

incurv'ifol'ia, Don = S. hirt'a, v. incurv'ifol'ia.

læ'tevir'ens, Don. p. 6.

leptophyll'a, Pers. p. 5.

muscoï'des, Wulf. p. 5. Mossy Alpine Saxifrage.

niva'lis, ${ }^{11}$ L. 14. p. 7, 8. Alpine Clustered Saxifrage.

oppos'itifol'ia, L. 28. p. 4, 5. Purple Montain Saxifrage.

ped'atif'ida, Sm. p. 5. Geranium-leaved Saxifrage. ${ }^{12}$

plat'ypet'ala, Sm. = S. hy'pnoí'des, v. plat'ypet'ala.

pygmæ'a, Haw. p. 5.

ri'vula'ris, ${ }^{13}$ L. 4. p. 7, 8. Alpine Brook Saxifrage.

sponhe'mica, ${ }^{14}$ Gmel. 10. p. 7 .

stella'ris, ${ }^{15}$ L. 38. p. 6, 7. Starry Saxifrage.

Sternberg'ii, ${ }^{16}$ Willd. I. p. 7. Irish Mossy Saxifrage.

tridact'yli'tes, L. 81. a. or b. 4-6. Rue-lcaved Saxifrage.

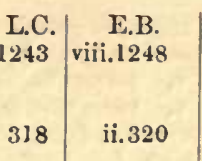

B.

191

ii. 197

131

iii.329

813

v. 703

554

543

iv. 551

iv. 549

iv. 556

548

546

iv. 554

550 iv. 557

539

iv. 543-5

iv. 543

$539 \mathrm{~b}$

$539 \mathrm{c}$

iv. 545

iv. 544

547

542

540

iv. 555

iv. 550

iv. 546

iv. 559

iv. 558

iv. 560

552 iv. 561,2

iv. 561

iv. 562

537

iv. $5+1$

iv. 540

545

iv. 553

(iv.560)

iv. 542

538

549

(iv.559)

544 iv.552
376

378

375

382

373

380

374

377

379

385

1 Serratala alpina. 2 Leiogyne aizoides. 3 L. cernua. 1 Rohertsonia Genm. 5 Kidney-shaped Saxifrage. 6 Lpíogyne granulata. 7 Hircalus rannenloides. 8 Robertsonia hirta. 9 Saxifraga incurvifolia. $10 \mathrm{~S}$. elingella.-S. platypetala. 11 Leingyne niralis. 12 Heb-foot-leaved Saxifrage. 13 Leiogyne rivularis. 14 Saxifraga aftinis, - S. hirta, v, affinis. I6 Robertsonia stellaris. 16 Saxifraga hirta. 
Saxifraga, L.-continued.

umbro'sa,' L. I. p. 6, 7. London Pride. None-so.Pretty. ${ }^{2}$

v. puncta'ta, Don.

v. serra'tifol'ia, Don.

Saxifrag'ex, Juss. Saxifrages.

Scabio'sa, L. Dipsa'ce. , Teazleworts. Tetran. Mon. arvens' is, ${ }^{3}$ L. 95. p. 7-9. Field Scabions or Knautia. Columba'ria, L. 71. p. 7-9. Small Scabious. marit'ima, L. C. sucei'sa, L. 112. p. 7-10. Devil's-bit Scabious.

Scand'ix, L. Umbellí'Er.e, Umbellifers. Pent. Dig. Ce'refol'ium, L. = Anthrisc'us Ce'refol'ium, Hoffm. Anthrise'us, $L .=$ Anthrisc'us vulga'ris, Bernh. od'ora'ta, L. $=$ Myrrh'is Od'ora'ta, Scop.

Pect'en-Ven'eris, L. 88. a. or b. 6.9. Venus' Comb.

Sche'donorus, Beaur.

ela'tior, Roem. \& Schult. = Festu'ca ela'tior, L.

lǒlia'ceus, Roem. \& Schult. = Festu'ca lǒlia'cea. pratens'is, Roem. \& Schult. = Festu'ca ela'tior, v. pratens'is. sylva'ticus, Roem. \& Schult. = Festu'ca sylva'tica, Vill.

Scheuchze'ria, L. NA'IAdA'Cex, Naiads. Hex. Trig. palust'ris, L. 6. p. 7. Marsh Scheuchzeria.

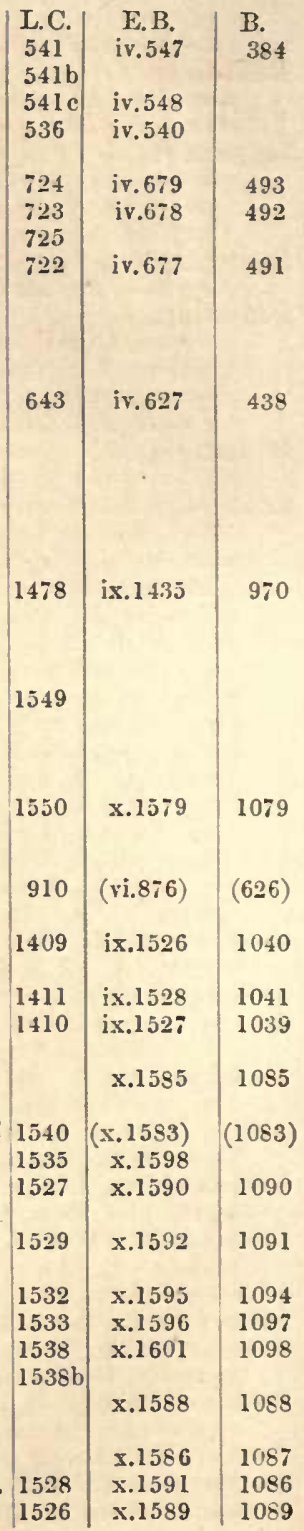

Scho'nus, L. CrP'Era'ce.e, Sedges. Tri. Mon. alb'us, L. = Rhȳnchosp'ora alb'a, Vahl. compress'us, L. = Scirp'us Ca'ricis, Retz. ferrugin'eus, L. 1 . fusc'us, L. = Rhỹnchosp'ora fusc'a, Roem. \& Schult. Marise'us, $\mathrm{I}_{\text {. }}=$ Clad'ium germa'nicum, Schrad. monoi'cus, Sm. = Kobre'sia ca'rici'na, Willd.

ni'gricans, L. 70. p. 6, 7. Black Schœenus or Bog-rush. ru'fus, Huds. = Scirp'us ru'fus, Schrad.

Scholle'ra, Roth. VAccíxis'ce. Cranberries. Oct. Mon. Oecycoec' us, ${ }^{4}$ Roth. 66. 8, 6-8. Marsh Whortleberry or Cranberry.

Scill'a, L. LI'LIA'CEs, Lilyworts. Hex. Mon. autumna'lis, L. 9. p. 7-9. Autumnal Squill. bifol'ia, L. p. 3, 4. Two-leaved Squill. nu'tans, ${ }^{5}$ Sm. 109. p. 4-6. Wood Hyacinth or Bluebell. vern'a, Huds. 26. p. 4, 5. Vernal Squill.

Scirp'us, L. Cyp'era'ce.e, Sedges. Tri. Mon. ac'icula'ris, L. = El'eoch'aris ac'icula'ris, Sm. ca'rici'nus, Schrad. = S. Ca'ricis, Retz. Ca'ricis, ${ }^{6}$ Retz. 51. p. 6, 7. Compressed Bog-rush. Broad-leaved carina'tus, Sm. 7. p. 7, 8. Trigonous-stemmed Bull-rush. crespito'sus, ${ }^{8}$ L. 101.p. 6, 7. Scaly-stemmed or Tufted Club-rush. cern'uus, Vahl. See Sa'vii, Seb. \& Maur.

flu'itans, ${ }^{9}$ L. 76. p. 6, 7. Flocting Club-rush or Water-rush. glauc'us, Sm. = S. Tabern'rmonta'ni, Gmel.

Hol'oschœ'nus, ${ }^{10}$ L. 1. p. 9. Round-headed or Clustered $C$. lacust'ris, L. 95. p. 7, 8. Bull-rush. Great or Lake C. marit'imus, L. 77. p. 6-8. Sea or Salt-marsh Club-rush. v. compact'us, Kioch.

multicau'lis, Sm. = El'eoch'aris multicau'lis, Sm.

na'nus, Spr. See parv'ulus, Roem. \& Schult.

palust'ris, $\mathrm{L}_{\mathbf{0}}=\mathrm{El}^{\prime}$ eoch'aris palust'ris, R.Br.

parv'ulus, ${ }^{11}$ Roem. \& Schult. [na'nus, Spr.]3.p. 7. Least Club-rush. pauciflo'rus, ${ }^{12}$ Lightf. 84. p. 7, 8. Chocolate-headed $C .^{13}$

1526 1 Robertsonia umbrosa. 2 St. Patrick's Cabbage. 3 Knatia arrensis. 4 Vaccinin
Agraphis nutans.-Endymion nutans.-Hyacinthus non-scriptus.

1 Vaccininm Oxycoceus.6 Blysmus Oxycoceus palnstris. 5 Agraphis nutans. - Endymion nutans.- H yacinthus non-scriptus. compressus, - Scirpus caricinns, - Schouns compressus.
caspitosus. i1 Eleocharis parrula. 12 E. pancifiora. 13 Few-flowered Club-rush. $10 \mathrm{I}$. Holoschœnus.-Holoschœus rulgaris. 
Scirp'us, L. - continued.

pung ${ }^{\prime}$ ens, ${ }^{1}$ Vahl. C. p. 6, 7. Leafy-stemmed or Sharp Club-rush.

ripa'rius, Spr. $=$ S. Sa'vii, Seb. \& Maur.

Rotb'ii, Hoppe. = S. pung'ens, Vahl. ru'fus, ${ }^{2}$ Schrad. 42. p. 7. Narrow-leaved Blysmus. Brown BogSa'vii, ${ }^{3}$ Seb. \& Maur. [cern'uus, Vahl.] 23. p. 7. Savi's Club-rush.

v. pygmae'us (Kunth)

seta'ceus, ${ }^{4}$ L. 100. p. 7,8. Bristle-stalked Club-rush. sylva'ticus, L. 70. p. 7. Wood Club-rush.

v. diss'itiflo'rus, Sonder.

Tabern'æmonta'ni,' Gmel. 47. p. 6-8. Glaucous Bull-rush.

trique'ter, L. 5. p. 10. Three-edged B. Triangular Club-rush. 1536

u'niglu'mis, Link = El'eoch'aris u'niglu'mis, Link.

Sclerranth'us, L. ILL'EcerRA'Ces, Knotworts. Dec. Dig. ann'uus, L. 95. 8. or b. 6-9. Commons Annual Knawel.

v. bienn'is (iReut.)

perenn'is, L. 5. p. 6-8. Perennial Knawel.

Scle'rochl'oa, Pal, de Beaur.

Burre'ri, Bab. = Glycer'ia Borre'ri, Bab.

dist'ans, Bab. = Glycer'ja dist'ans, Wahl.

lǒlia'cea, Woods = Festu'ca lǒlia'cea.

marit'ima, LindI. = Glycer'ia marit'ima, Wahl.

multiculm'is, Syme.

procumb'ens, P. de B. = Festu'ca procumb'ens, Kunth.

rig'ida, Link = Festu'ca rig'ida, Kunth.

Scŏlopend'rium, Sm. Fil'ices, Ferns. Crypt. Fil.

Cet'erach, Symons = Cet'erach offic'ina'rum, Willd.

vulga're, Symons. 96. p. 7, 8. Hart's-tongue Fern.

Scro'phula'ria, Tourn. Scro' PHularis'e.e, Figworts. Did. Ang. ala'ta, Gilib. = S. umbro'sa, Dum.

aqua'tica, ${ }^{8}$ L. 70. p. 7, 8. Common Water Betony.

v. Balbi'sii, Hornem.

v. ciner'ea, Dum.

Balbi'sii, Horn. = S. aqua'tica, L.

Ebrbart'i, Stev. = S. umbro'sa, Dum.

nodo'sa, L. 106. p. 7-10. Knotted-rooted Figwort. Steven's $F$. Scŏrodo'nia, L. 4. p. 7, 8. Balm-leaved Figurort.

umbro'sa, ${ }^{7}$ Dum. 18. p. 8, 9. Ehrhart's Water Betony.

verna'lis, L. p. 4-6. Y'llow Figwort.

Scro'phularin'ex, Juss. Figworts.

Scu'tella'ria, L. LAd'1s't

gale'ricula'ta, L. 97. p. 7-9. Common Seull-cap.

min'or $\times$ galéricula'ta, Nich.

min'or, Huds. 66. p. 7-10. Lesser Scull-cap.

Sed'um, L. Crassula'ce., Honseleeks. Dec. Pent. [pepper. a'ere, L. 104. p. 6, 7. Biting Stone-crop, or Golden Moss. Wallalbesc'ens, Haw. =S. reflex'um, v. aJbesc'ens.

alb'um, L. p. 7, 8. White Stone-crop.

v. ter'etifol'ium ${ }^{8}$ (Haw.)

v. micranth'um ${ }^{9}$ (Bast.)

an'glicum, Huds. 55. p. 6-8. White English, or Mountain S. dasyphyll'um, L. p. 6, 7. Thiek-leaved White Stone-crop.

e'legans, Lej. = S. rupest're, L.

Faba'ria, Koch $=\mathrm{S}$. Teleph'ium, v. Faba'ria.

Forste'ria'num, Sm. 11. p. 6, 7. Forster's Stone-crop. ${ }^{10}$

v. glaucesc'ens, Lond. Cat.

v. viresc'ens, Lond. Cat.

glatc'um, Sm. = S. reflex'um, v. albesc'ens.

1 Scirpos Rothii. 2 Blysmus rufus.-Sehonus rufus, 4 Isulepis setacea. 5 Scirpas glaucus. 6 Scrophularia Babisii teretifolium. 9 . micranthum. 10 Welsh Rock Stone-crop. [rush.

L.C.

1537

E.B.

$\mathrm{x} .1600$

1541

1530

$1530 \mathrm{~b}$

1531

1539

$1539 \mathrm{~b}$

1534

B.

1095

1093

(x.1584)

(1084)

$\mathrm{x} .1593$

\begin{tabular}{l|l}
$\mathrm{x} .1594$ & 1092
\end{tabular}

$\begin{array}{ll}\mathrm{x} .1602 & 1099\end{array}$

X.1597

x.1599

1096

x.1587

1176 vii.1174

$1176 \mathrm{~b}$ vii.1175

834

$117 \%$

vii. 1176

835

xi. 1756

xi. 1755

xi.1759

xi. 1754

xi. 1755,6

xi. 1757

xi. 1758

lis2 xii.1884

1296

1039

vi. $9+7$

740

$1039 a$

$1039 b$

1041

vi. 948

vi. 949

vi. 950

739

1042

$\begin{array}{rr}1040 & \text { (vi.945) } \\ 1043 & \text { vi.95l }\end{array}$

1021 vi.937

1130 vii. 1060

vii.1060 793

1130

1131 vii.1061

794

iv. 532

iv. 535

iv. 529

365

565

363

$565 a$ (iv. 529.1)

565 b (iv.529.2)

567 iv. 531

361

566 iv. 530

362

iv. 536

iv. 527

$5 \% 2$

$572 \mathrm{a}$

iv. 537

$572 \mathrm{~b}$

3 Isolepis Snvii.-Bcirpus riparius. 7 S. alata, -S. Ehrharti. 8 Sedum 
Sed'um, L.-continued.

micranth'um, Bast. = S. alb'um, v. micranth'um.

purpurasc'ens, Koch $=\mathrm{S}$. Teleph'ium, v. purpurasc'ens.

reflex'um, L. p. 7, 8 . Crooked Yellow Stone-crop.

v. albesc'ens ${ }^{1}$ (Haw.) Glaucous Yellow Stone-crop.

Rhod'iola, ${ }^{2}$ DC. [ros'eum, Scop.] 37. p. 5-8. Rose-root Stone-crop. ros'eum, Scop. See Rhod'iola, DC.

rupest're, ${ }^{3}$ L. 11. p. 6, 7. St. Vincent's Rock Stone-crop.

v. ma'jus, Syme.

v. $\min ^{\prime} u s$, Syme.

sexang'ula're, L. p. 7. Insipid or Tasteless Yellow S.

Teleph'ium, L. 75. p. 7, 8. Live-Long or Everlasting Orpine.

v. purpurasc'ens, ${ }^{4}$ (Koch) Broad-leaved Orpine.

v. Faba'ria ${ }^{5}$ (Koch) 13. Narrow-leaved Orpine.

ter'etifol'ium, Haw. = S. alb'um, v. ter'etifol'ium.

villo'sum, L. 29. b. 6, 7. Hairy Stone-crop.

562 iv. 525

563 iv. 526,7 sela'ginoi'des, ${ }^{6}$ Gray. 55. p. 7, 8. Lesser Alpine Clubmoss. spi'nulo'sa, A.Br. = S. sela ginoïdes, Gray.

Sela'Ginella'Cex.

Seli'num, L. Umbellif'ers, Umbellifers. Pent. Dig. Carvifol'ia, I. 2. p. 7, 8. Carraway-leaved Marsh Parsley. palus'tre, $L_{.}=$Peuc'edan'um palust're, Moench.

Sempervi'vum, L. Crassula'ce e, House-leeks. Dod. Dod. tecto'rum, L. p. 6, 7. House-leek.

Senebie'ra, DC. Crocif'ers, Crucifers. Tetrad. Silic. Coro'nopüs, ${ }^{7}$ Poir. 81. a. 6-9. Swine's-cress. Wart-cress.

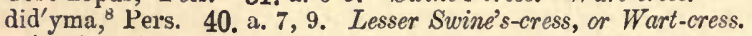

Senec'io, L. Compos'itx, Composites. Syn. Sup. aqua'ticus, Huds. 110 . b. or p. 7, 8. Marsh Ragwort. v. pinnātif'idus, Gren. \& Godr.

campest'ris, ${ }^{\theta}$ DC. 21 . b. or p. 5, 6. Field Fleawort. eru'cifol'ius, ${ }^{10}$ L. 67. p. 7, 8. Hoary Ragwort. inte'grifol'ia, With. = S. campest'ris, DC.

Jac'obæ'a, L. 112. p. 6-9. Common Ragwort. v. flosculo'sus, (Jord.)

li'vidus, Sm. = S. sylva'ticus, v. aur'icula'tus. paludo'sus, L. 3. p. 6, 7. Great F'en R. Bird's-tongue $R$. palust'ris, ${ }^{11}$ DC. 7. b. 6, 7. Marsh Fleawort. sarace'nicus, L. p. 7, 8. Broad-leaved Ragwort. spath'ulæfol'ius, DC. 2. p. or b. 6, 7 . squa'lidus, L. a. or b. 6.10. Inelegant Ragwort. sylva'ticus, L. 104. a. 7-9. Mountain Groundsel. v. aur'icula'tus, ${ }^{12}$ W. Meyer.

ten'uifol'ius, Jacq. = S. eru'cifol'ius, L.

visco'sus, L. 28. a. 7, 8. Stinking Gronundsel. vulga'ris, L. 112. a. 1-12. Common Groundsel. v. radia'tus, Koch.

\section{Sera'pias, L.}

ensifol'ia, L. = Ceph'alanthe'ra ensifol'ia, Rich.

grandiflo'ra, Lightf. = Ceph'alanthe'ra pall'ens, Rich.

la'tifol'ia, L. = Fipipact'is la'tifol'ia, Auct.

palust'ris, Lightf. = Épipact'is palust'ris, Crantz.

ru'bra, L. = Ceph'alanthe'ra ru'bra, Rich.

Serrafalc'us, Purl.

arvens' is, Godr. = Brom'us arvens' is, $\mathbf{L}$.

commuta'tus, Bab. = Brom'us commuta'tus, Schrad.
L.C. E.B.

iv. 529,2

iv. 526

570 iv. 534

$570 \mathrm{~b}$ (iv.535)

571

(iv.536)

$571 \mathrm{a}$

$571 \mathrm{~b}$

569

563a (iv.526)

$563 \mathrm{~b}$ (iv.527)

iv. 529,1

1564 iv. 528

364

1828 xii.1829

$(1258)$

B.

359

367

366

360

1828

663

573

iv. 538

368

138 i. 160

107

i. 159

108

787

$787 \mathrm{~b}$

791

v.756

543

v.760

549

v.754

545

786

v.755

544

$786 \mathrm{~b}$

789

790

v.758

546

v.759

548

v. 757

547

792

784

v.753

542

v. 750

541

v. 751

783

v. 752

540

781

v. $7 \pm 9$

539
1 Sednm albescens.-S. glancum.

2 Rhodiola rosea. 9 Cineraria campestris,-Senecio integrifolia.

3 Sedum elegans. -Lepidium didymum. 11 Cineruria palustris.

12 Senecio lividus.

4 S. pnrparascens. 8 C. didyma. $10 \mathrm{~S}$. tenuifolius. 
Serrafalc'us, Parl.-continued.

moll' is, Parl. = Brom'us moll'is, L.

pat'ulus, Parl. = Brom'us pat'ulus, Mert. \& Koch.

rac'emo'sus, Parl. = Brom'us rac'emo'sus, "L."

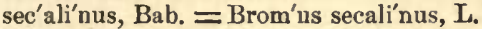

squarro'sus, Parl. = Brom'us squarro'sus, $\mathbf{L}$.

Serra'tula, I. Compos'ıтs, Composites. Syn. Æq. alpi'na, L. = Saussu'rea alpi'na, DC.

tinctória, L. 64. p. 8. Common Saw-wort.

v. montic'ola (Boreau)

Ses'eli, L. Umbellif'́le, Umbcllifers. Pent. Dig. Libano'tis, ${ }^{1}$ Koch. 3. b. or p. ? 7, 8. Montain Meadow Saxifrage. ${ }^{2}$

Sesle'ria, Scop. Grämin'e.e, Grasses. Tri. Dig. carul'ea, Ard. 10. p. 4-6. Blue Moor-grass.

v. flavesc'ens, Moore.

Seta'ria, Beauv. GrÄmí'ex, Grasses. Tri. Dig. glauc' $a,{ }^{3}$ Beauv. a. 9. Glancous Bristle-grass. vert'icilla'ta, ${ }^{4}$ Beauv. a. 7, 8. Rongh $B$. Rough Panick-grass. vir'idis, ${ }^{5}$ Beauv. 31. a. 7, 8. Green B. Green Panick-grass.

\begin{tabular}{l|c|} 
L.C. & E.B. \\
& \\
& \\
& \\
& \\
814 & v. 704 \\
814 b & v.704 bis \\
\end{tabular}

B.

647

iv. 602

1688 xi. 1710

426

$1688 b$

1630

1631

1629

xi. 1694

(1156)

xi. 1693

(1157)

709 iv. 663

480

320

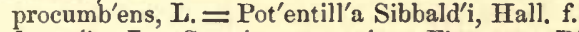

Sibthorp'ia, I. Scro'pudlarix'Ex, Figworts. Did. Ang. europæ'a, L. 7. p. 7-10. Cornish Moneywort. Creeping Sibthorpia.

1046

vi. 969

745

Siegling'ia, Bernh. Grämin'E£, Grasses. Tri. Dig. decumb ens, ${ }^{7}$ Bernh. 103. p. 7. Decumbent Heath Grass.

Sil'ăus, Bess. Umbellif'ers, Umbellifers. Pent. Dig. flavesc'ens, Bernh. See pratens'is, Bess. pratens'is, ${ }^{8}$ Bess. [flavesc'ens, Bernh.]

[Sulphur-wort. ${ }^{9}$

Sile'ne, L. CAR'yophrL'Ez, Cloveworts. Dec. Trig. acau'lis, L. 21. p. 6-8. Moss Campion. Citshion Pink. alpest'ris, Jacq. Alpine Catchfly.

an'glica, I. = S. gall'ica, v. an'glica.

Arme'ria, L. a. 7, 8. Common Garden or Lobel's Catchfly.

co'nica, L. 10. a. 5-7. Striated Campion or Corn Catchfly.

co'noí'dea, I, a. 7. (Lindl. Syn.)

Cucu'balus, ${ }^{10}$ Wibel. 102. p. 6-8. Bladder Campion. White Bottle. v. puber'ula, Syme.

diurn'a, Gren. \& Godr. = Lych'nis diurn'a, Sibth.

gall'ica, L. 57. a. 6-10.

v. an'glica ${ }^{1 i}$ (L.) English Catchfly or Campion.

v. ros'ea, Melvill.

v. an'glico $\times$ quinquevul'nera, Melvill.

infla'ta, Sm. = S. Cucu'balus, Wibel.

ital'ica, ${ }^{12}$ Pers. 4. p. 6, 7. Italian Catchfly. Dover Catchfly. marit'ima, With. 75. p. 6-8. Sea Bladder Campion or Catchfly. noctiflo'ra, I. 43. a. 7, 8. Night-flowering Catchfly.

nu'tans, L. 15. p. 5-7. Nottingham Catchfly.

Oti'tes, ${ }^{13}$ Sm. 3. p. 6-8. Spanish Catchfly.

pat'ens, Peete $=$ S. ital'ica, Pers.

pratens'is, Gren. \& Godr. = Lych'nis alb'a, Mill.

quinquerul'nera, I. C. a. 6, 7. Spotted or Variegated $C$.

Sil'er, Scop. Umbellif'er.e, Umbellifers. Pent. Dig. tril'obum, Scop. p. 5, 6.

Sil'ybum, Vaill. Coupos'ır e, Composites. Syn. Aq. Maria'num, ${ }^{14}$ Gærtn. b. 7-9. Milk Thistle. $1686(x i .1745)$

660

iv. 604

428

198

ii. 205

132

ii. 202

ii. 204

194

ii.201

137

192

$192 b$

(ii.199)

ii.211

196 ii. 202,3

$196 \mathrm{a}$ (ii.202)

$196 \mathrm{~b}$

$196 \mathrm{c}$

201

193

202

200

199

ii. 199

133

ii. 208

ii. 200

ii. 209

ii. 207

138

135

ii.206

134

ii. 210

197

ii.203

659

812

v. 681

(555)
1 Athamanta Libanotis.- Lihanotis montans.

4 P. rerticillatum.

decumbens. 5 P. viride.

- Spur

2 Mountain Stone Parsley. Bilene inflata.
8 Cuidium Silaus.-Peucedanum Silaus. $11 \mathrm{~S}$. anglica. $12 \mathrm{~B}$. patens.
Little Field Madder. 9 Pepper Saxifrage. 13 Cucubalus Otites.

3 Panicum glancum. Pos decumbens. - Triodia 10 Cucubsins Behen.4 Carduas Marianus. 
Sime'this, Kunth. Ll'LIA'Cex, Lilyworts. Hex. Mon. bicol'or, Kunth. 1. p. 6. Variegated Simethis.

Sina'pis, L.

alb'a, L. = Brass'ica alb'a, Boiss.

arvens'is, $\mathrm{L}$. = Brass'ica Sina'pis, Visiani.

Cheiranth'us, lioch = Brass'ica Cheiranth'us, Vill.

inca'na, L. = Brass'ica adpress'a, Boiss.

mura'lis, R. Br. - Diplotax' is mura'lis, DC.

ni'gra, L. = Blass'ica ni'gra, Koch.

ten'uifol'ia, Sm. = Diplotax'is ten'uifol'ia, DC.

Si'son, L. Umbellif'eræ, Umbellifers. Pent. Dig.

Amo'mum, L. 53. b. 8, 9. Hedge-Stonewort. Honewort. ${ }^{1}$

inunda'tum, L. $=A p^{\prime}$ 'ium inunda'tum, Reichb.

seg'etum, L. = Car'ım seg'etum, Benth.

vert'icilla'tum, L. = Car'um vert'icilla'tum, Koch.

Sisymb'rium, L. Crocif'ere, Crucifers. Tetrad. Siliq.

Allia'ria, ${ }^{2}$ Scop. 94.a.5,6. Garlic Hedge Mustard. Jaek-bythe-Hedge. ${ }^{3}$

amphib'ium, L. = Nasturt'ium amphib'ium, R.Br.

I'rio, L. 1. a. 7, 8. London Rocket. Broad Hedge Mustard. monens'e, L. = Brass'ica monens'is, Huds.

mura'le, $\mathbf{L}$. = Diplotax' is mura'lis, DC.

Nasturt'ium, L. = Nasturt'ium offic'ina'le, R.Br.

offic'ina'le, ${ }^{4}$ Scop. 107. a. 6, 7. Common Hedge Mustard. panno'nicum, Jacq.

pol'yeerat'ium, L. a. 7, 8. Prostrate Hedge Mustard.

Soph'ia, L. 64. a. 6-8. Fine-leaved H. M. Flix-weed.

sylvest're, I. = Nasturt'ium sylvest're, R.Br.

ten'uifol'ium, L. = Diplotax'is ten'nifol'ia, DC.

terrest're, $\mathrm{Sm}_{.}=$Nasturt'ium palust're, DC.

'Thălia'num, ${ }^{8}$ J. Gay. 93. a. 4, 5. Thale-cress. Wall-cress. ${ }^{6}$

Sisyrinch'ium, L. InID'ese, Irids. Tri. Mon.

$a^{\prime}$ ceps, Bab. $=\mathrm{S}$. angust'ifol'ium, Mill.

angust'ifol'ium, ${ }^{7}$ Mill. I. p. 7 . Blue-eyed Grass. ${ }^{8}$

Bermu'dia'na, L. $=$ S. angust'ifol'ium, Mill.

Si'um, L. UMbelilif'ere, Umbellifers. Pent. Dig.

angust'ifol'ium, $L .=S$, erect'um, Huds.

erect'um, ${ }^{9}$ Huds. 76. p. 7, 8. Narrow-leaved Water-Parsnip. inunda'tum, Wigg. = Ap'ium inunda'tum, Reichb.

la'tifol'ium, L. 40. p. 7, 8. Great or Broad-leaved W. P. no'diflo'rum, $\mathrm{L} .=A p^{\prime}$ ium no'difo'rum, Reichb.

re'pens, L. = Ap'ium no'dito'rum, v. re'pens.

vert'icilla'tum, Lam. = Car'um vert'icilla'tum, Koch.

Smi'laci'na, Desf.

bifol'ia, Desf. = Maianth'emum Conralla'ria, Web.

Smyrn'ium, L. Unmellif'Ere, Umbellifers. Pent. Dig.

Olusa'trum, L. 62. b. 4-6. Common Alexanders.

So'taANA'CEA, Juss. Nightshades.

Sola'num, I. So'LANA'Ce. Niqhtshades. Pent. Mon. Dulcama'ra, L. 96. p. 6-8. Woody Night-shade. Bitter-Sweet. v. mari'num, Bab.

v. o'chroleuc'um, auct.

ni'grum, L. 61. a. 7-10. Common Night-shade. Black N.10 v. minia'tum (Bernh.) v. lu'teo-viresc'ens (Gmel.)

Solida'go, L. Compos'rte, Composites. Syn. Sup. camb'rica, Iluds. = S. Virgaur'ea, v. camb'rica.

I Hedge Bastard Stone Parsley. Sauce Alone. 4 Erysimum officinale. Bermudiana.-S. anceps. Nightshade.
2 Alliaria officinalis.-Erysimnm Alliaria.

5 Arabis Thaliana. 6 Turkey Pod.

8 Blue Sisyrinchium. 9 Sium angustifolium.
3 Hedge Garlic.-

7 Sisyrinchium jo Garden 
Solida'go, L.-continued.

Virgaur'ea, L 108. p. 7-9. Common Golden-rod.

v. angust'ifol'ia, Gaud.

v. camb'rica ${ }^{1}$ (Huds.)

Sonch'us, L. Compos'it.玉, Composites. Syn. Aq.

alpi'nus, L. = Lactu'ca alpi'na, Benth.

arvens'is, L. 107. p. 8, 9. Corn Sow-thistle.

v. gla'bra, Lond. Cat.

v. angust'ifol'ia, Mey.

asp'er, Hoffm. 96, a. 6-8. Rongh or Sharp. fringed Annual Sowcrerul'eus, Sm. = Lactu'ca alpi'na, Benth.

olera'ceus, L. 109. ̊. 6-9. Common Smooth Sow-thistle.

thistle. palust'ris, L. 7. p. 7-9. Tall Marsh Sow-thistle.

Sorb'us, L.

Aucupa'ria, L. = Pyr'us Aucupa'ria, Gærtn.

Spargan'ium, Tourn. 'TŕpHA'CEÆ, Bulmushes. Monœc. Tri.

atfi'ne, ${ }^{2}$ Schnizi. 19. p. 7. Floating Bur-rced.

min'imum, ${ }^{3}$ Fr. 46. p. 7, 8. Small Bur-reed.

na'tans, Bab. = S. aft'ne, Schnizl.

na'tans, $\mathrm{L} .=\mathrm{S}$. min'imum, Fr.

neglect'um, Beeby. 9."

ramo'sum, Huds. $18 .^{4}$ p. 6, 7. Branched Bur-reed

sim'plex, Huds. 92. p. 6, 7 . Unbranched Upright Bur-reed.

L.C.

Townsend'i, H. \& J. Grores. 1. Intermediate Cord-grass.

Spart'ium, L.

scopa'rium, L. $=$ Cyt'isus scopa'rius, Link.

Spěcula'ria, L. Campa'sura'ces, Bellworts. Pent. Mon.

hyb'rida, ${ }^{6}$ DC. 47. a. 6-9. Small-flowered Venus'-Looking-glass. ${ }^{7}$

Sperg'ula, L. CAR' Yopnýl'es, Cloveworts. Dec. Pent.

arvens'is, L. 111. a. 6-8. Corn Spurrey, or Yarr.

v. vulga'ris ${ }^{\triangleleft}$ (Boenn.)

v. sati'va (Boenn.)

niva'lis, Lindb. = Sagi'na niva'lis, Fr.

nodo'sa, L. = Sagi'na nodo'sa, Mey.

pentand'ra, $\mathrm{Sm} .=\mathrm{S}$. arvens'is, $\mathbf{v}$. vulga'ris.

sagi'noi'des, L. = Sagi'na Linnæ'i, Presl.

su'bula'ta, Sw. = Sagi'na su'bula'ta, Wimm.

Spergula'ria, Pers.

mari'na, Camb. = Lepigo'num margina'tum, Kocls.

margina'ta, Syme = Lepigo'num margina'tum, línch.

med'ia, Garcke = Lepigo'num margina'tum, Koch.

neglect'a, Syme = Lepigo'num sali'num, Fr.

ru'bra, Fenzl. = Lepigo'num ru'brum, Fr.

rupest'ris, Lebel. = Lepigo'num rupest're, Kindb.

sali'na, Presl. = Lepigo'num sali'num, Fr.

Spiræ'a, L. Rosa'cers, Roseworts. Icos. Pent.

Fi'lipend'ula, L. 61. p. 6, 7. Conmon Dropwort.

sal'icifol'ia, L. 112, s. 7, 8. Willow-leaved Spircea or Dropwort.

Ulma'ria, L. 112.p.6-8. Meadow Sweet. Queen of the Meadows.

v. de'nuda'ta, Presl.

Spiranth'es, Rich. Orcin'Ex, Orchids. Gyn. Mon.

iestiva'lis, ${ }^{9}$ Rich. 2. p. 7, 8. Summer Lady's-tresses.

autumna'lis, ${ }^{10}$ lich. 57. p. 8, 9. Autumnal or Fragrant $L$.

ccrn'ua, Rich. = S. Romanz'oria'na, Cham.
727

$727 \mathrm{~b}$

$727 \mathrm{c}$

891

$891 \mathrm{~b}$

$891 \mathrm{c}$

890

889

892

1460

1461

1458

1457

1459

1633

1632

1634

E.B.

v. 778

B.

500

v.779

r. 813

591

588

v. 811,2

พ. 810

590

v. 814

589

ix. 1389

ix. 1390

940

ix. 1387

- 938

ix. 1388

939

xi. 1688

xi.1687

1197

909

(vi.874)

(622)

247

$2+7 \mathrm{a}$

ii. 252,3

ii. 253

ii. 252

170

ii.257

ii. 255

ii. 254

ii. 256

415

iii. 416

301

413

iii. 414

299

iii. 415

¿00

$414 \mathrm{~b}$

1333

ix. 1473

989

1332

1 Solidago cambrica. 2 Sparganium natans, Bub. 3 S. natans, L. 4 Agg. neglectum \& ramosum 104. 5 Dactylis stricta. 6 Campanula hybrida. - Prismatocarpus hybridus. 7 Corn Campanula or Bellylower. 8 Spergula peutandra. 9 Seottia astivalis. $10 \mathrm{~N}$. spiralis.-Ophrys spiralis. 
Spiranth'es, Rich.-continued. gemmip'ara, Lindl. = S. Romanz'ovia'na, Cham. Romanz'ovia'na, ${ }^{1}$ Cham. I. p. 8,9. Three-ranked Lady's-tresses. ${ }^{2}$

Spi'rode'la, Schleid. polyrrhi'za, Schleid. = Lemn'a polyrrhi'za, $\mathrm{L}$.

Stach'ys, L. LAB'14'Ts, Labiates. Did. Gym. ambig'ua, ${ }^{3}$ Sm. 38. p. 9, 10. Ambiguous Woundwort. ${ }^{4}$ ann'ua, L. a. 9, 10. Pale Annual Woundwort. arvens'is, L. 95. a. 4-11. Corn Woundwort. Beto'nica, ${ }^{6}$ Benth. 81. p. 6-8. Wood Betony. germa'nica, L. 3. p. or b. 8. Downy Woundwort. palust'ris, L. 109. p. 7-9. Marsh Woundwort. sylva'tica, L. 108. p. 9, 10. Hedge Woundwort. sylva'tici-palust'ris, Wirtg. $=$ S. ambig'ua, Sm.

Stăphyl'ea, L. Sтт̆PHÝ'Ex, Bladder-Nuts. Pent. Trig. pinna'ta, L. s. 6. Common Bladder-Nut.

Stat'ice, L. Plumbagis'ex, Leadworts. Pent. Pent. arme'ria, L. = Arme'ria marit'ima, Willd.

auric'ulafol'ia, ${ }^{6}$ Vahl. 22. p. 7, 8. Lesser or Rock Sea-Lavender. ${ }^{7}$

v. oc' cidenta'lis $^{8}$ (Lloyd.)

v. intermed'ia, Syme.

v. Dodart'ii ${ }^{9}$ (Gir.)

bahu'siens'is, Fr. = S. rariflo'ra, Drej.

Be'hen, Drej. = S. Limo'nium, v. genui'na.

v. py'ramida'lis $=\mathrm{S}$. Limo'nium, v. py'ramida'lis.

bellidifol'ia, Gouan $=\mathrm{S}$. re'ticula'ta, $\mathrm{Sm}$.

binervo'sa, G. F. Sm. = S. auric'ulæfol'ia, Vahl.

v. oc'cidenta'lis $=\mathrm{S}$. auric'ulæfol'ia, v. oc'cidenta'lis.

v. Dodart'ii $=$ S. auric'ulæfol'ia, v. Dodart'ii.

casp'ia, Willd. = S. re'ticula'ta, Sm.

Dodart'ii, Gir. = S. auric'ulæfol'ia, v. Dodart'ii. [ [avender. ${ }^{10}$ Limo'nium, L. 35. p. 7.11. Great or Spreading Spiked Seav. genui'na, ${ }^{\prime \prime}$ Syme.

v. py'ramida'lis, ${ }^{12}$ Syme.

marit'ima, Sm. = Árme'ria marit'ima, Willd.

oc'cidenta'lis, Lloyd $=$ S. auric'ulæfol'ia, v. oc'cidenta'lis. plantāgin'ea, All. = Arme'ria plantāgin'ea, Willd.

rariflo'ra, ${ }^{23}$ Drej. 22. p. 7, 8. Remote-flowered Sea-Lavender. re'ticula'ta, ${ }^{14}$ Sm. 6. p. 7, 8. Matted Sea-Lavender.

spăthula'ta, Desf. $=$ S. auric'ulæful'ia, Valıl.

Stella'ria, L. CAR'Yophyl'Es, Cloveworts. Dec. Trig. aqua'tica, ${ }^{15}$ Scop. 55. p. 7, 8. Water Chickweed. cerast'oï'des, L. = Cerast'ium trigyn'um, Vill.

glauc'a, With. =S. palust'ris, Ehrh.

grāmin'ea, L. 107. p. 5-8. Lesser Stitchwort or Starwort.

Holost'ca, I. 106. p. 4-6. Greater Stitchwort. Starwort. med'ia, Cyr. 112. a. 4-10. Common Chickweed or Stitchwort.

v. Boræa'na (Jord.)

v. neglect'a (Weilie)

nem'orum, L. 44. p. 5-8. Wood Chickweed or Stitchwort. palnst'ris, ${ }^{16}$ Ehrh. 48. p. 5-7. Glaucons Marsh Stitchwort. scapig'era, Willd. p. 6. Many-stalked Starwort. üli'gino'sa, Murr. 107. a. 5-7. Bog or Fountain Stitchwort. umbro'sa, Opiz. 18. a.

L.C.

1334

ix. 1474

(ix.1474)

1138

1141

1140

1135

1136

1137

1139

(vii.1070)

vii.1073

vii.1072

vii. 1067

vii. 1068

vii. 1069

vii. 1071

vii. 1070

ii.32.?

936

(vii.1159, 60)

936a (vii.1159)

$936 \mathrm{~b}$

$936 \mathrm{c}$

vii. 1158
vii. 1156

vii.1157

vii.1159,60

vii.1159

vii.1160

vii.1161

934 vii.1156-8

934a (vii.1156)

934b (vii.1157)

820

935

(vii.1158)

937 (vii.1161)

822

162

221

ii. 227

167

ii. 231

227

225

223

$223 \mathrm{~b}$

$223 \mathrm{c}$

222

226

228

224

B.

801

797

798

800

799

821

ii. 232

ii. 230

ii. 229

166

168

164

ii. 228

(ii. 231)

163

(167)

ii. 233

165

1 Neottia cernua.-N. gemmipara.-Spirantbes cernua.-S. gemmipara.

2 Drooping or Proliferous Lady's-tresses.
Betonica officinalis.
S 6 Statice binervosa_-S. spatbulata. Hybrid betueen Hedge \& DInrsh Woundunts. Lavender. 8 Statice occidentalis. $\mathrm{S}$. binervosa, $\mathbf{v}$. oceidentalis. 9 S. Dodartii. - S. binervosa, $\checkmark$. Dodartii. 10 Lavender Thrift. 11 Statice Behen. $12 \mathrm{~S}$. Behen, v. pyramidalis. $13 \mathrm{~S}$. bahnsiensis. $14 \mathrm{~S}$. bellidifolin.-8. caspia. 15 Cerastium aquaticum.-Malacbium aquaticum.

17 Larbrea aquatica. 
Sti'pa, L. “GRIuin'ex, Grasses. Tri. Dig. atio'tes, L. Hr'drocharid'es, Hydrocharads. Polyan. Pent. aloi'des, L. 15. p. 6-8. Water Aloe. Water Saldier.

Sturm'ia, Reicbb.

Loese'lii, Reichb. = Lip'aris Loese'lii, Rich.

Suæ'da, Forsk. Che'nopod'1u'Cex, Chenopods. Pent. Dig. firutico'sa, ${ }^{1}$ Forsk. 7. s. 7-10. Shrubby Sea-blite, Saltwort, or Goosefoot.
marit'ima, ${ }^{2}$ Dum. 63, a. 7-10. Annual Sea-blite. Sea Goosefoot. ${ }^{3}$

E.B.

B. v. procumb'ens, Syme.

Su'bula'ria, L. Crucrf'er., Crucifers. Tetrad. Silic. aqua'tica, L. 21. p. 6-8. Water Awl-wort.

Swert'ia, L. Gextina'ce.e, Gentianworts. Pent. Dig. perenn'is, L. p. 8. Marsh Felwort.

Sym'phytum, Tourn. Borĩarn'es, Borageworts. Pent. Mon. offic'ina'le, L. 85. p. 5, 6. Common Comfrey. v. pat'ens (Sibth.) tubero'sum, L. 25. p. 6, 7. Tuberous Comfrey.

'AAM'ARISCIN'E.e, Desv, Tamarisks.

Tam'arix, L. 'Tasi'ariscin'Ex, Tamarisks. Pent. Trig. an'glica, Webb $=$ T. gall'ica, L. gall'ica, ${ }^{+}$L. s. 7-9. English or French Tamarisk.

Tam'us, L. Dioscor'ex, Yams. Diœc. Hex. conmu'nis, L. 69. p. 5, 6. Black Bryony.

Tan'ace'tum, I. Compos'rte, Composites. Syn. Sup. vulga're, ${ }^{5}$ L. 102. p. 8, 9. Common Tansy.

Tarax'acum, Juss. Coxpos'1t. Dens-leo'nis, Desf. = T. offic'ina'le, Web.

offic'ina'le, ${ }^{6}$ Web. 110. p. 3-10. Common Dandelion.

v. Dens-leo'nis, Desf.

v. ery'throsperm'um (Andrz.)

v. palust're ${ }^{7}$ (DC.) Marsh Dandelion.

v. u'dum (Jord.)

palust're, $\mathrm{DC} .=$ T. offic'ina'le, v. palust're.

Tax'us, Tourn. Conif'er.e, Conifers. Diœc. Monad. bacea'ta, L. 48. t. 3, 4. Common Yew.

v. fasti'gia'ta ${ }^{8}$ (Lindl.) Irish Yew. Florence Court Yew. fasti'gia'ta, Lindl. = Tax'us bacca'ta, v. fasti'gia'ta.

Teesda'lia, R.Br. Crucir'er.e, Crucifers. Tetrad. Silic. Ibe'ris, DC. = T. nu'dicau'lis, R.Br. nu'dicau'lis, ${ }^{9}$ R.Br. 70. a. 4-6. Naked-stalked Teesdalia.

Teuc'rium, L. LAB'in't.e, Labiates. Did. Gym. Bot'1'ys, L. 1. a.8. Cut-leaved Germander. Chame'drys, L. s. 7-9. Wall Germander. scord'ioï'des, Schreb. p. 7, 8.

Scord'ium, L. 10. p. 7, 8. Water Germander.

Scórodónia, L. 108. p. 7-9. Wood Sage or Germander.$$
986
$$

256

ii. 261

ii.261

256 (ii.261)

174

1388

ix. 1508

1023

770 (

532

592

882

v.802

$882 a$

$882 \mathrm{~b}$

v. 803

$882 \mathrm{c}$
$882 \mathrm{~d}$

v. 804

1320 viii. 1384

935

$1320 \mathrm{~b}$

.

149

i. 150

98

1156

vii.1091

814

1158

vii. 1094

815

1157

vii. 1092

813

1159

vii.1093

812

Thalict'rum, L. Ranunc'ula'ces, Crowfoots. Polyan. Pol. alpi'num, L. 26. p. 7, 8. Alpine Meadow Rue. ${ }^{10}$ fla'vum, L. 65. p. 7, 8. Common or Yellow Meadov Rue.

v. sphærocarp'um, Lej.

v. ripa'rium (Jord.)

v. ni'gricans, Jacq.

flexuo'sum, Bernh. p. 6, 7. Zigzag Meadow Rue.

Koch'ii, Fr. = T. saxa'tile, v. Koch'ii.

1 Chenopodium frutirosum.- Salsola fruticosa. 2 Chenopodium maritimnm. 3 Herbaceous Suada. 7 Leontodon palustre.-Taraxacum palustre. 8 Taxus fastigiata. 9 Iberis nudicalis.-Teesdalia Iberis

10 Poor Man's Rhubarb. 
Thalict'rum, L.-continued.

ma'jus, Sin. p. 7, 8. Great Meadow Rue.

min'us, L. 65. p. 7, 8. Lesser Meadow Rue.

v. marit'imum, Syme.

v. monta'num, Wallr.

v. flexuo'sum (Reichb.)

saxa'tile, DC. p. 6, 7. Stone Meadow Rue.

v. Koch'ii ${ }^{1}$ (Fr.) Koch's Meadow Rue.

Thēsi'um, L. Sartala'cee, Sandalworts. Pent. Mon. hŭmifu'sum, IC. = T. lin'ophyll'um, L.

hum'ile, Vahl. p. 7, 8. Erect Bastard Toad-flax.

lin'ophyll'um, ${ }^{2}$ L. 22.p.5-7. Lint-leaved Bastard T. Flax-leaved T.

Thlasp'i, L. Crucif'era, Crucifers. Tetrad. Silic.

alpest're, L. 14.p.6-8. Alpine Shepherd's Purse. Corn Penny Cress.

r. sylvest'1 ' ${ }^{3}$ (Jord.) Short-styled Alpine Penny Cress.

v. occita'num ${ }^{4}$ (Jord.) Long-styled Alpine Penny Cress.

v. vir'ens ${ }^{5}$ (Jord.) Green Alpine Penny Cress.

arvens'e, L. 79. p. 5-7. Field P. Mithridate Mustard.

Burs'a-pasto'ris, L. = Capsell'a Burs'a-pasto'ris, Moench.

campest'ris. L. = Lepid'ium campest're, R.Br.

hirt'um, Sm. = Lepid'ium Smith'ii, v, a'latosty'la.

occita'num, Jord. ='T. alpest're, v. occita'num.

per'folia'tum, L. 3.a. 4, 5. Perfoliate Penny Cress or Shepherd's. Purse.

sylvest're, Jord. $=$ T. alpest're, v. sylvest're.

virens, Jord. $=$ T. alpest're, v. vir'ens.

Thrinc'ia, Roth.

hirt'a, Roth. = Leont'odōn hirt'us, L.

THYM'ELAA'CEE, Juss. Daphnads.

Thym'us, L. LAR'1A'rs, Labiates. 1)id. Gym.

$A_{c}^{\prime}$ inos, L. = Cal'aminth'a arrens'is, Lam.

Cal'aminth'a, Sm. = Cal'aminth'a offic'inna'lis, Moench.

Chamæ'drys, Fr. 40. p. 7, 8. Large Wild Thyme.

Nep'eta, Sm. = Cal'aminth'a Nep'eta, Clairv.

Serpyll'um, Fr. 111. p. 6-8. Creeping Wild Thyme.

Til'ia, L. Tília'cese, Lindenblooms. Polyan. Mon.

corda'ta, ${ }^{6}$ Mill. 18. t. 7, 8. Small-leaved Lime-tree.

europæ'a, L. = T. vulga'ris, Hayne.

grandifol'ia, Ehrh. = T. platyphyll'os, Scop.

intermed'ia, $\mathrm{DC}$. $=\mathrm{T}$. vulga'ris, Hayne.

parvifol'ia, Ehrh. $=\mathrm{T}$. corda'ta, Mill.

platyphyll'os, ${ }^{7}$ Scop. 3. t. 6, 7. Large-leaved Downy Lime-tree. ru'bra, DC. (Lindl.) t. 8.

vulga'ris, ${ }^{8}$ Hayne. t. 7. Common Lime. Linden-Tree.

TrLIA'CEe, Juss. Lindenblooms.

Tillæ'a, L. Crassula'ce.s, Houseleeks. Tetran. Tetrag. musco'sa, L. 8. a. 6, 7. Mossy Tillaa. Mossy Red-Shanks.

Tofield'ia, Huds. 'Lr'LiA'CEs, Lilyworts. Hex. 'Trig. palust'ris, Huds. 15. p. 7, 8. Montain Scottish Asphodel.

Tol'ypell'a, Leonh. CharA'Cese, Charads. Crypt. Char. glom'era'ta, ${ }^{8}$ Leonh. 20. a. or p. 4. Clustered Nitella. intrica'ta, ${ }^{10}$ Leonh. 9, a. 4, 5. Many-fruited Nitella. nidif'ica, ${ }^{11}$ Leonh. I. prolif'era, ${ }^{12}$ Leonh. 4. a. Many-fruited Nitella.

Tordyl'ium, L. Umbellif'er.e, Umbellifers. Pent. Dig. max'imum, L. 4. a. 6, 7. Great Hart-wort. offic'ina'le, ${ }^{13}$ L. 2. 6, 7. Small Hart-wort.

\begin{tabular}{|c|c|c|}
\hline L.C. & E.B. & B. \\
\hline 3 & i. 3,4 & 3 \\
\hline $3 \mathrm{a}$ & i. 3 & \\
\hline $3 b$ & i. 4 & \\
\hline $3 c$ & & \\
\hline $\begin{array}{l}4 \\
4 \mathrm{~b}\end{array}$ & i. 7 & \\
\hline $4 \mathrm{~b}$ & $(1.6)$ & \\
\hline & viii. 1248 & \\
\hline 1243 & (viii. 1248) & 880 \\
\hline 147 & i. $146-8$ & 97 \\
\hline $147 a$ & (i.146) & \\
\hline $147 \mathrm{~b}$ & (i.147) & \\
\hline $147 c$ & (i.l 48) & \\
\hline 145 & i. 144 & 95 \\
\hline & i. 147 & \\
\hline 146 & $\mathrm{i}, 145$ & 96 \\
\hline & $\begin{array}{l}\text { i.146 } \\
\text { i. } 148\end{array}$ & \\
\hline & $\mathrm{v}^{1.148}$ & \\
\hline 1239 & viii. 1246 & \\
\hline 1119 & vii. 1044 & \\
\hline 1118 & vii. $10+3$ & 785 \\
\hline 285 & (ii.287) & \\
\hline & ii.285 & 199 \\
\hline & ii. 286 & \\
\hline & ii. 287 & \\
\hline 283 & (ii.285) & \\
\hline 284 & (ii.286) & (199) \\
\hline 283 & ii. 285 & \\
\hline 560 & iv. 524 & 357 \\
\hline 1423 & ix. 1543 & 1053 \\
\hline 1849 & (xii.1905) & \\
\hline 1851 & (xii.1907) & \\
\hline 1848 & & \\
\hline 1850 & (xii.1908) & \\
\hline 671 & iv. 614 & 437 \\
\hline
\end{tabular}

1 Thalictrum Kochii. 2 Thesium humifusum. 3 Thlaspi sylvestre. 4 T. accitanum. 5 T. virens. 6 Tilia parvifolia. 7 T. grandifolia. 8 T. europa.,-T. intermedia. 9 Chara glomerata,-C. nidifiea.C. Smithii,-Nitella glomerata. 10 Chara intricata,-C. polysperma,-Nitella intricata. $11 \mathrm{~N}$. nidifica. 12 Chara Borreri.-C. prolifera, -Nitella intricata, v, prolifera.-N, prolifer a. 13 Condylocarpas officinalis. 
Tor'ilis, Hoffm.

Anthrisc'us, Gmel. = Cauc'alis Anthrisc'us, Huds.

infest'a, Spr. = Cauc'alis arvens'is, Huds.

nodo'sa, Gærtn. = Cauc'alis nodo'sa, Scop.

Tormentill'a, Tourn.

'ffic'iña'lis, Sm. = Pot'entill'a Tormentill'a, Neck.

rept'ans, L. = Pot'entill'a procumb'ens, Sibtb.

Trăgopo'gōn, L. Coмpos'rт e, Composites. Syn. Aq.

min'or, Fr. $=$ 'T. pratens'is, v. min'us.

porrifol'ius, I. b. 5, 6. Salsify. Purple Goat's-beard.

pratens'is, L. 83. b. 6, 7. Yellow Goat's-beard.

r. min'us $^{1}$ (Mill.)

v. grandiflo'rus, Syme.

Tricho'dium, Mich.

cani'num, Schrad. = Agro'stis cani'na, L.

seta'ceum, Roem. \& Schult. = Agro'stis seta'cea, Curt.

Trichom'anes, L. Fin'ices, Ferns. Crypt. Fil.

brevise'tum, R.Br. =T. radi'eans, Sw.

radi'cans, ${ }^{2}$ Sw. 4. p. 9, 10. Rooting Bristle-fern.

v. Andrews'it, Newm.

Trichone'ma, Ker.

Bulboco'dium, Sm. = Romul'ea Colum'næ, Seb. \& Maur.

Colum'næ, Reichb. = Romul'ea Colum'næ, Seb. \& Maur.

Trienta'lis, L. PrímULA'Ces, Primworts. Hept. Mon.

europæ'a, L. 34. p. 6, 7. European Chickweed Winter-green.

Trifol'ium, L. Legu'Mivo'se, Leguminous Plants. Diad. Dec. agra'rium, L. a. 6.

arvens'e, L. 92. a. 7-9. Hare's-foot Trefoil.

Bocco'ni, Savi. 1. a. 7. Boccone's Trefoil.

dub'ium, ${ }^{3}$ Sibth. 100. a. 6-8. Lesser Yellow Trefoil.

fi'liform'e, L. 61. a. 6, 7. Slender or Least Yellow Trefoil.

fragif'erum, L. 70. p. 7, 8. Strawberry-leaved Trefoil.

glom'era'tum, L. 19. a. 6. Smooth Round-headed Trefoil.

hyb'ridum, L. p. 6-8. Alsike Clover.

v. élegans (Savi.)

incarna'tum, L. 2. 6, 7. Crimson Clover.

marit'imum, Huds. [squamo'sum, L.] 23, a. 6-8. Teasel-headed Trefoil.

med'ium, L. 99. p. 6-9. Zigzag T. Meadow Clover. Cow-grass. $\min ^{\prime}$ us, Sm. $=$ T. dub'ium, Sibth.

Moline'rii, Balb. 1. a. 6, 7. Balbi's Trefoil.

o'chroleuc'on, Huds. 11. p. 6.8. Sulphur-coloured Trefoil.

offic'īna'le, Sm. = Melilo'tus altiss'ima, Thuill.

orni'thopod'ioi'des, Sm. = Trig'onell'a purpurasc'ens, Lam.

pratens'e, L. 112. p. or b. 5-9. Red Clover. Purple Trefoil. Marl-grass."

v. sativum, Syme.

v. sylvest're, Syme.

v. parviflo'rum, Bab.

procumb'ens, L. 101. a. 6-8. Hop Trefoil.

re'pens, I. 112. p. 5-10. White Clover. Dutch C. Trefoil. v. Townsend'ii, Bab.

res'upina'tum, L. a. 7. Reversed-flowered Trefoil.

sca'brum, L. 47. a. 5-7. Rough, Rigid, or Hard Knotted T.

L.C. E.B.

B.

894

v. 801

576

893

v.798

$893 \mathrm{~b} \quad \mathrm{v.799}$

.575

$893 \mathrm{c}$

v. 800

1763

$1763 \mathrm{~b}$

95

954

361

350

351

363

364

359

354

357

$357 \mathrm{~b}$

347

346

344

348

345

343

$3+3 \mathrm{a}$

$343 \mathrm{~b}$

$343 \mathrm{c}$

362

358

$358 b$

360

353

349

352

$352 b$ xii. 1839

1304

ix. 1492

vii.1139

1016

655

iii.354

iii. 355

(iii.366)

iii.367

iii. 363

iii. 358

iii.361

iii. 352

iii. 350

iii. 348

iii.366

iii. 353

iii. 349

244

251

(262)

263

258

254

260

243

249

248

262

246

iii. $3+7$

247

iii. 365

261

iii. $362 \quad 259$

iii. 364

iii. 357

256

252

iii. 351

245

iii. 356

250

stria'tum, L. 70, a. 6, 7. Soft Knotted Trefoil.

v. erect'um, Leight. 
Trifol'ium, L-Continued.

strict'um, L. 2. a. 6, 7. Upright Round-headed Trefoil. subterra'neum, L. 39. a. 5, 6. Subterranean Trefoil. suffoca'tum, L. 14, a. 6, 7. Dense-flowered, Suffocated, or Sand T.

Triglo'chin, L. Na'IADA'CE.E, Naiads. Hex. Trig. marit'imum, L. 76. p. 5-9. Sea-side Arrow-grass. palust're, L. 106. p. 6-8. Marsh Arrow-grass.

Trig'onell'a, L. LEGU'Mino's s, Leguminous Plants. Diad. Dec. orni'thopod'ioi'des, DC. =T. purpurasc'ens, Lam. purpurasc'ens, ${ }^{1}$ Lam. 29.a.or b.6-8. Bird's-foot Fenugreek or Clover.

Tri'nia, Hoffin. UMbellif'ere, Umbellifers. Pent. Dig. glaber'rima, Hoffm. = T. vulga'ris, DC. vulga'ris, ${ }^{2}$ DC. 3. b. 5, 6. Glabrons Stonewort or Honewort. ${ }^{3}$

L.C.

356

342

355

1477

1476 od'ia, R.Br. decumb'ens, P. de B. = Siegling'ia decumb'ens, Bernh.

Trise'tum, Kunth. GRĀmix'en, Grasses. Tri. Dig. flavesc'ens, ${ }^{4}$ Beauv. [pratens'e, Pers.] 88. p. 7, 8. Yellow Oat-grass. pratens'e, Pers. See flavesc'ens, Beauv. pubesc'ens, Roem. \& Schult. = Ave'ua pubesc'ens, Huds.

Tri'ticum, L. acu'tum, DC. = A'gropy'ron acu'tum, Gren. \& Godr. ? cani'num, Huds. $=A^{\prime}$ gropy'ron cani'num, Beauv. crista'tum, Schreb. = A'gropy'ron crista'tum, Schreb. junc'eum, L. = A'gropy'ron junc'eum, Beauv. lax'um, Fr. = A'gropy'ron acu'tum, Gren. \& Godr. ? lǒlia'ceum, Sm. = Festu'ca lŏlia'cea. pung'ens, Koch. $=A^{\prime}$ gropy'ron pung'ens, Roem. \& Schult. re'pens, $\mathbf{L}$. $=\mathbf{A}^{\prime}$ gropy'ron re'pens, Beauv.

Troll'ius, L. Ranunc'ula'ces, Crowfoots. Polyan. Pol. europæ'us, L. 61. p. 6-8. Mountain Globe-flower.

Tu'lipa, Tourn. Lília'ces, Lilyworts. Hex. Mon. sylvest'ris, L. p. 4, 5. Wild Tulip.

Turri'tis, Dill. gla'bra, L. = Ar'abis per'folia'ta, Lam. hirsu'ta, Sm. = Ar'abis sagitta'ta, DC.

Tussila'go, L. Compos'its, Composites. Syn. Sup. alpi'na, L. p. 4. Alpine Coltsfoot. Farf'ara, L. 112. p. 3, 4. Common Coltsfoot. fra'grans, Vill. = Pětasi'tes fra'grans, Presl. byb'rida, $\mathrm{L}_{\text {. }}=$ Pětasi'tes vulga'ris, Desf. Pětasi'tes, L. = Pětasi'tes vulga'ris, Desf.

Ty'pha, Tourn. T'Trpha'ces, Bulrushes. Monce. Tri. angust'ifol'ia, L. 57. p. 7. Narrow-leaved Cat's-tail or Reedmace. ${ }^{5}$ la'tifol'ia, L. 79. p. 7, 8. Great C. or R. Bulrush Cat's-tail. v. med'ia, Syme.

min'or, Sm. p. 7. Dwarf Reedmace or Bulmush.

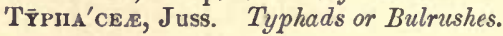

U'lex, L. Legu'nino's.e, Leguminous Plants. Diad. Dec. europæ'us, L. 110, s. 2-6. Furze. Whin. Gorse. v. strict'us ${ }^{6}$ (Mackay) Irish Furze.

Gall'ii, Planch. 53. s. 8-11. Planchon's Frurze. na'nus, Forster. 24. s. 8, 9. Dwarf Furze. strict'us, Mack. $=U$ U. europæ'us, v. strict'us.

Ulm'us, L. Urtícs'ce.z, Nettle-worts. Pent. Dig. campest'ris, Sm. t. 3-5. Common Elm. v. su'bero'sa, ${ }^{7}$ Elirh. Cork-barked Elm. v. gla'bra, Mill. Smooth Elm. Cornish Elm.

E.B.

iii. 360

iii. 346

iii. 359

B.

253

257

255

ix. 1434

972

971

iii. $345 \quad 242$

330

(iii. 345)

(242)

iv. 579

405

xi. 1745

1247

(xi.1736)

xi. 1812

xi. 1809

$x i .1813$

xi.1811

xi. 1810

ix. 1385

iii. 324

325

iii. 325

1 Falcatula ornithopodioides.-Trifolinm ornithopodioides.-Trigonella ornithopodioides. glaberrima. - Pimpinella dioica. 3 Dwarf Burnet Saxifrage. I Avena flavescens. 5 Lesser Bulrush. * Ulex strictus. 7 Ulmus suberosa. 8 U. carpinifolia.-U. glabra.-U. stricta.-U. suberosa, v. glabra. 
Olm'us, L.-continued.

carpi'nifol'ia, Lindl. $=$ U. campest'ris, v. gla'bra.

gla'bra, Sm. = U. campest'ris, v. gla'bra.

ma'jor, Sm. $=$ U. monta'na, v. ma'jur.

[Hazel. ${ }^{2}$

monta'na, Stokes. 93. t. 3, 4. Broad-leaved Elm. Wych Elm, or

v. ma'jor ${ }^{2}$ (Sm.) Dutch Elm.

v. nit'ida, Syme.

strict'a, Lindl. = U. campest'ris v, gla'bra.

su'bero'sa, Ehr. = U. campest'ris, v. su'bero'sa.

v. gla'bra $=\bar{U}$. campest'ris, v. gla'bra.

UMBELLIF'ERA, JusS. Umbellifers.

Umbili'cus, DC. Crassula'ce.e, Houseleeks. Dec. Pent. erect'us, DC. p. 7. penduli'nus, DC. = Cot'yle'don Umbili'cus, $\mathbf{L}$.

Urti'ca, 'Tourn. URTícs'ces, Nettleworts. Monœc. Tet. dioic'a, L. 112. p. 6-9. Common Nettle. Great Nettle. v. angust'ifol'ia, $\mathbf{A}$. Blytt.

Dodart'ii, L. $=$ U. pilulif'era, v. Dodart'ii.

pilulif"era, L. a. 6-8. Roman Nettle.

v. Dodart'ii ${ }^{3}$ (L.) Dodart's Nettle.

u'rens, L. 107. a. 6-9. Small Nettle.

UrTícs'cex, Juss. Nettleworts.

J'tricula'ria, L. Lent'ibula'rie e, Butterworts. Dian. Mon. Bre'mii, Heer. p. Heer's Bladderwort.

intermed'ia, Hayne. 19. p. 8. Intermediate Bladderwort. ${ }^{4}$

min'or, L. 68. p. 6-8. Lesser Bladderwort.

neglect'a, Lehm. 11. p. 6-8. Lehman's Bladderwort.

vulga'ris, L. 82. p. 6-8. Greater B. Hooded Water Milfoil.

VACcinin'cex, DC. Cranberries.

Vaccínium, L. VAccinia'ces, Cranberries. Oct. Mon. mäcrucarp'um, Ait.

Myrtill'us, L. 100.s. 4-6. Bilberry. Blaeberry. Black WhortleOxycocc'os, L. = Scholle'ra Oxycocc'us, Roth.

ũli'gino'sum, L. 17. s. 5, 6. Great Bilberry. Bog Whortleberry. Vi'tis-Idæ'a, L. 62, s. 6, 7. Red Whortleberry. Cowberry.

Valer'ia'na, L. VALER'1A'NEx, Valerianworts. Tri. Mon. denta'ta, Ehrh. = Valer'ianell'a denta'ta, Poll.

dioic'a, L. 69. p. 5, 6. Small Marsh Valerian.

Locust'a, L. = Valer'ianell'a olito'ria, Moench.

offic'inn'lis, L. 111. p. 6-8. Great Wild Valerian. All-heal.

v. Mika'nii, Syme. 12.

v. sambu'ciful'ia, ${ }^{5}$ Syme.

pyre'na'ica, L. p. 6, 7. Heart-leaved Valerian.

ru'bra, L. = Centranth'us rub'er, DC.

sambu'cifol'ia, Mik. = V. offic'ina'lis, v. sambu'cifol'ia.

VALER'IA'NEA, Juss. Valerianworts.

Valer'ianell'a, Tourn. VALER'IA'NEe, Valerianworts. Tri. Mon. Auric'ula, ${ }^{6}$ DC. 29. a. 6-8. Sharp-fruited Lamb's-Lettuce or Corn-salad. denta'ta, ${ }^{3}$ Poll. 79. я. 6-8. Smooth Narrow-fruited $L$. or $C$. v. mixt'a, ${ }^{9}$ Dufr

er'iocarp'a, Desv. 5. a. 6, 7. Hairy-fruited Lamb's-Lettuce. mixt'a, Iufr. $=$ V. denta'ta, v. mixt'a. olito'ria, ${ }^{10}$ Moench. 95. b. 4-6. Common Lamb's-Lettuce, or Corn-

Vell'a, L. Crucir'teis, Crucifers. Tetrad. Siliq. ann'ua, ${ }^{11}$ L. a. 6. Annual Cress Rocket.

1 Mountain or Scotch Elm. 2 Ulmas major. 3 Urtica Dodurtii. 5 Valeriana sambucifolia. 6 Fedia Anricala. 5aleriana sambucifolia, ${ }^{6}$ Fedia Anricala. 7 F. carinata. 8 F. dentata
Valerianella mixta. 10 Fedia olitoria.-Valeriana locusta, II Carrichtera Vella.

- Middle Hooded Water Milfoil. 
Verbasc'um, L. Scro'pholarix'ex, Figworts. Pent. Mon.

Blatta'ria, L. b. or a. 6-10. Moth Mullein.

\begin{tabular}{|c|c|c|} 
L.C. & E.B. & B. \\
1026 & vi.942 & 724 \\
& & \\
1027 & vi. $943-6$ & \\
1027 a & vi.943 & \\
1027 b & vi.944 & \\
1027 c & vi.945 & \\
1027 d & vi.946 & \\
1023 & vi.939 & 727 \\
1024 & vi.940 & 726 \\
1022 & vi.938 & 728 \\
1021 & vi.937 & 723 \\
1025 & vi.941 & 725
\end{tabular}

flocco'sum, Waldst. \& Kit. = V. pulv'erulent'um, Vill.

hyb'ridi :
a. Thaps'o-Lychni'tis. b. 8.
b. Thaps'o-ni'grum. b. 8 .
c. ni'gro-pulv'erulent'um. p. 8.
d. ni'gro-Lychni'tis. p. 8.

Lychni'tis, L. 12. b. 7, 8. White Mullein.

ni'grum, L. 42. p. or b. 6-10. Dark or Black Mullein.

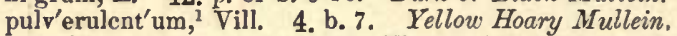

thaps'iform'e, Schrad. b. 7, 8. Thapsus-like Millein.

Thaps'us, L. 90. b. 6-8. Great Mullein. High Taper.

virga'tum, With. b. or a. 8. Large-flowered Primrose-leaved $M$.

Verbe'na, Tourn. Verbenu'ces, Verbenes. Did. Gym.

offic'ina Tis, L. 67. p. 7-9. Vervain.

Verbena'cexe, Juss. Verbenes.

1101

vi.1018

1101 vi. 1018

Ve'roni'ca, Tourn. Scro'phularin'es, Figworts. Dian. Mon.

agrest'is, L. 107. a. 4-9. Green Procumbent Field Speedwell. ${ }^{2}$ alpi'na, L. 8. p. 7, 8. Erect Alpine Speedwell.

1050

Anagall'is, ${ }^{3}$ Jacq. 97. p. 7, 8. Long-leaved Water Speedwell. Anagall'is-aqua'tica, L. $=$ V. Anagall'is, Jacq.

arvens'is, L. 109. a. 5-10. Wall Chickweed Speedwell.

v. exim'ia, 'Towns.

Beccabung'a, L. 110.p. 5-9. Short-leaved Water S. Brooklime.

Buxbaum'ii, Ten. $=\mathrm{V}$. pers'ica, Poir.

Chamæ'drys, L. 111. p. 5, 6. Germander Speedwell.

frut'icans, Jacq. See saxa'tilis, Scop.

frut'iculo'sa, L. p. 7. Flesh-coloured Speedwell. Shrubby S.

hed'eræfol'ia, I. 96. a. 3-8. Ivy-leaved Chickweed Speedwell.

hirsn'ta, Hopk. $=$ V. offic'inna'lis, v. hirsu'ta.

hŭmifu'sa, Dicks. $=$ V. serpyll'ifol'ia, v. hŭmifu'sa.

hyb'rida, ${ }^{4}$ L. 9. p. 7, 8. Spiked or Welsh Speedwell.

monta'na; L. 86. p. 6-9. Mountain Speedwell.

offic'īna'lis, L. 111. p. 5-7. Common Speedwell.

v. hirsu'ta ${ }^{5}$ (Hopkirk) Hairy Speedwell.

peregri'na, L. a. 5-10. Purslane S. Annual Smooth S.

pers'ica, ${ }^{6}$ Poir. 80. a. 4-9. Buxbaum's Speedwell.

poli'ta, Fr. 87. a. 4-9. Grey Procumbent Chickweed $S$.

v. grandifo'ra, Bab.

re'pens, DC. p. 4,5. Creeping Speedwell.

saxa'tilis, Scop. [frut'icans, Jacq.] 7. s. 7-9. Blue Rock Speedwell. scu'tella'ta, L. 100. p. 7, 8. Narrow-leaved Marsh Speedwell.

v. parmula'ria, Tur. et Poit.

serpyll'ifol'ia, L. 111. p. 5, 6. Perennial Smooth S. Thymeleaved $S_{0}^{7}$

v. hŭmifu'sa ${ }^{8}$ (Dicks.) 12. Prostrate Smooth Speedwell. spica'ta, L. 3. p. 7, 8. Spiked or Blunt-fingered S.

v. hyb'rida $=\mathrm{V}$. hyb'rida, $\mathrm{L}$.

triphyll'os, L. 6. a.5, 6. Upright or Trifid Chickweed Speedwell. vern'a, L. 4. a. 5, 6. Vernal Chickweed Speedwell.

1058

1066

1054

$1054 \mathrm{~b}$

1067

1063

vi.972

vi. 980

vi.989

749

752

vi.976

760

vi.990

753

vi. 973

759

vi. 986

756

1048 vi.970

757

vi. 979

1061

1064

1062

$1062 \mathrm{~b}$

1055

1051 (vi.97i)

1049 vi.971

$1049 \mathrm{~b}$

1057

1059

(vi.983)

vi. 987

vi. 984

755

vi.985

vi. 977

1065

vi. 981

748

vi. 988

754

1056

vi. 978

$1056 \mathrm{~b}$ (vi.979)

1060 vi.982

vi. 983

vi.974

vi. 975

750

747

1052

1053

urn' um, L. CAP'RIFOL'IA'CE E, Caprifoils. Pent. Trig.

Lanta'na, L. 43. s. 5, 6. Wayfaring Tree. Mealy Guelder-Rose."

Op'ulus, L. 75. s. 6, 7. Common Guelder-Rose. Water Elder.

686

iv. 640

761

685

iv. 639

461

392 iii.393, 4

$392 \mathrm{a}$ iii. 393

$392 b$ iii.394

angust'ifol'ia, L. 87. a. 5-7. Narrow-leaved crimson Vetch. ${ }^{10}$

v. segeta'lis (Thuill.)

v. Bobart'ii ${ }^{11}$ (Forster)

1 Verbascum floccosum.

4 . spicata, v. bybrida.

S Meal Tree.
2 Germander Chichweed. $\nabla$. hirsuta.
Wild Vetch. 
Vic'ia, L. -continued.

bithy'nica, L. 17. p. 5, 6. Rough-podded purple $V$. Bithynian $V$.

v. la'tifol'ia, Syme.

v. angust'ifol'ia, Syme.

Bobart'ii, Forst. $=$ V. angust'ifol'ia, v. Bobart'ii.

Cravc'a, I. 112. p. 6-8. Thefted Vetch.

grac'ilis, Lois. 20. a. 6-9. Many-seeded Slender Tare.

hirsu'ta, ${ }^{1}$ Koch. 104. a. 5-8. Hairy Tare.

byb'rida, L. p. 6, 7. Hairy-flowered Yellow Vetch.

læviga'ta, Sm.p. 7, 8. Smooth-podded Sea Vetch.

lath'yroï'des, L. 52. a. 5, 6. Spring Vetch.

lu'tea, L. 16. p. 6-8. Rough-podded Yellow Vetch.

Ur'obus, ${ }^{2}$ DC. 30. p. 6-9. Wood Bitter Vetch.

sati'va, L a. 4-6. Common Cultivated Vetch. Tare.

se'pium, L. 105. p. 5-7. Common Bush Vetch.

sylva'tica, L, 72. p. 6, 7. Wood Vetch.

tet'rasperm'a, ${ }^{3}$ Mœncb. 72. a. 5-8. Four-seeded Slender Tare.

Villars'ia, Vent.

nymiphæo'oï'des, Vent. = Limnanth'emum pelta'tum, Gmel. .

Vinc'a, L. A P'ocrsa'ces, Dogbanes. Pent. Mon. major, L. s. 4, 5. Greater Periurinkle.

min'or, L. 71. s. 4, 5. Lesser Periwinkle.

Víola, L. Viola'rie.z, Violetworts. Pent. Mon.

arēna'ria, DC. 2. b. 5, 6. Sand Dog Violet.

arvens' is, Murr. 110.4 a. 7.9. Small-flowered Field or Corn Pansy.

cani'na, ${ }^{5}$ L. 77. p. 4-8. Dillenius's Dog Violet.

Curti'sii, Forster. 17. p. 7-9. Yellow Sea-side Pansy.

fa'vicorn'is, $\mathrm{Sm} .=\mathrm{V}$. cani'na, $\mathrm{L}$.

hirt'a, L. 71. p. 4-6. Hairy Violet.

v. calca'rea, Bab.

v. sciaphil'a (Koch)?

L.C
39
39

.

lact'ea. Sm. 15. p. 6. Smith's Dog Violet. Cream-coloured $V$. v. intermed'ia, H. C. Wats.

lu'tea, IIuds. 62. p. 5-9. Yellow Monntain Violet or Pansy.

v. amc'na, Syme.

od'ora'ta, I. 79. p. 3-5. Sweet Violet.

v. alb'a. A uet.

v. permixt'a ${ }^{6}$ (Jord.)

จ. sepin'cola (Jord.)

palust'ris, L. 103. p. 4-7. Marsh Violet.

permixt'a, Jorl. $=\mathbf{V}$. od'ora'ta, v. permixt'a.

pers'icifol'ia, ${ }^{7}$ Roth. 4. p. 5, 6. Haller's Dog Violet.

pu'mila, Vill. $=$ V. cani'na L.

Reichenba'chia'na, Bor. 40. p. 4-6. Reichenbach's Dog Violet.

Ri'vinia'na, Reich. $=\mathbf{V}$. sylva'tica, Fr.

stagni'na, Kit. $=$ V. pers'icifol'ia, Roth.

sylva'tica, ${ }^{3}$ Fr. 112. p. 3-7. Wood or Hedge Violet. Gerard's Dog Violet.

tricol'or, L. $110 .{ }^{9}$ a. 5-9. Large-flowered Field Pansy. ${ }^{10}$

VIOLA'RIEA, DC. Violet-worts.

Visc'um, L. Lo'raxtin'Ce.e, Loranths. Dice. Tet. alb'um, L. 40. s. 3-5. Common Mistletoe.

Vulp'ia, Gmel.

bromoï'des, Dum. = Festu'ca sciu'roïdes, Roth.

myu'rus, Gmel. = Festu'ca myu'rus, L.

u'niglu'mis, Dum. = Festu'ca u'niglu'mis, Soland.
3948

$394 \mathrm{~b}$

384

383

381

390

389

393

388

385

391

387

386

382

963

964

169

174

170

175

166

$166 \mathrm{~b}$

$166 \mathrm{c}$

E.B.

iii. 396

B.

285

iii. 385

iii. 384

iii. 382

iii. 391

iii. 390

iii.395

iii. 389

iii. 386

iii. 392

iii. 388

iii. 387

iii. 383

278

276

284

282

280

283

281

279

277

vi.905

669

vi.906

670

ii. 174 bis

ii. 179

(ii.175)

ii. 180

ii. 175

ii. 172

122

171

$171 b$

176

$176 \mathrm{~b}$

165

$165 \mathrm{~b}$

$165 \mathrm{c}$

$16 \mathrm{Jd}$

164

ii. 170

120

$-172$

(ii.177)

168

ii. 174

ii. 173

ii. 177

167

173

(ii.173)

ii. 178

124

164

ii. 170

1242 iv. 635 bis

1 Errom hirsutum. 2 Orobus sylvaticos.

3 Ervim tetraspermn

4 Including Vinla tricolor. V. flavicornis, - V. pumila. V. permixta. 10 Pansy Violet. - Three-coloured Heart's-ease.

9 Including V. arvensis. 
Wahlenberg'ia, Schrad. Campa'nula'ces, Bellworts. Pent. Mon.

hĕdera'cea, ${ }^{1}$ Reichb. 45. p. 7,8. Ivy-leaved Bell-flower.

Wolff'ia, Horkel. Lemna'cen, Lemnads. Dian. Mon. arrhi'za, ${ }^{2}$ Wimm. 4. p. Rootless Duckweed.

Woods'ia, R.Br. Fin'ices, Ferns. Crypt. Fil. alpi'na, Gray $=$ W. hyp'erbor'ea, R.Br. hyp'erbor'ea, ${ }^{3}$ R.Br. 4. p. 7, 8. Round or Broad-leaved Alpine ilvens' is, R.Br. 8. p. 7-9. Oblong-leaved Woodsia.

Xanth'ium, Tourn. Compos'rтs, Composites. Monœc. Pent. spino'sum, L. Spiny Clot-bur. struma'rium, L. a. 8, 9. Broad Bur-Marigold. Bur-weed.

Zann'ichell'ia, L. NA'rads'ces, Naiads. Monoc. Mon. brăchyste'mōn, J. Gay. 14." ma'croste'mōn, Gay. Long-stemmed Horned Pondweed. palust'ris, L. 70. a. 5-8. Common Horned Pondweed. pedi'cella'ta, Fr. $=$ Z. pedunc'ula'ta, Reichb. pedunc'ula'ta, ${ }^{5}$ Reichb. 14. ${ }^{6}$ a. or p. Stalked-fruited Horned $P$. polycarp'a, Nolte. 1. a. 5-8. Short-styled Horned Pondweed. v. tenuiss'ima, Fr.

Zoste'ra, L. NA'IAdA'CEx, Naiuds. Monce. Mon. mari'na, L. 52. p. 7-9. Broad-leaved Grassurack. v. angust'ifol ia, Fr. na'na, Roth. 16. p. 7-9. Dwarf Grasswrack.

1 Campanula hederacea.

2 Lemna arrhiza. 4 Including Zannichellia pedunealata. $5 \mathrm{Z}$. pedicellata.

\begin{tabular}{|l|c|c} 
L.C. & E.B. & B. \\
898 & (vi.875) & $(621)$ \\
1469 & $($ ix.1398) & $(947)$ \\
& & \\
1784 & xii.1863 & \\
1783 & xii.1862 & 1303 \\
& & \\
754 & & \\
753 & v.860 & 517 \\
& & \\
1511 & & \\
1510 & ix.1425 & 951 \\
1512 & ix.1426 & \\
(ix.1426) & \\
1513 & & \\
$1513 b$ & & \\
1514 & ix.1429 & 948 \\
$1514 b$ & ix.1430 & \\
1515 & ix.1431 & 949
\end{tabular}

3 Woodsia alpina -Polypodium hyperboreum. 6 Including Z. brachystemon. 


\section{ENGLISH AND LATIN NAMES.}

Aaron's Beard. Hypericum calycinum. Abele-tree. Populus alba.

Absinth. Artemisia Absinthium.

Aconite. Aconitum Napellus.

Adder's-tongue. Ophioglossum.

Agrimony. Agrimonia.

Alder. Almus glutinosa.

Alehouf. Nepeta Glechoma.

Alexanders. Smyrnium Olusatrum.

Alkanet. Anchusa.

All-good. Chenopodium Bonus-Henricus.

All-heal. Valeriana officinalis.

All-seed. Chenopodium polyspermum.

All-seed. Polycarpon tetraphyllum.

All-seed. Radiola linoides.

Alpine Club-moss. Selaginella selaginoides.

Amarantl. Amaranthus Blitum.

American Cress. Barbarea pracox.

Anemone. Anemone.

Angelica. Archangelica officinalis.

Angelica. Angelica sylvestris.

Apple. Pyrus Malus.

Archangel. Lamium.

Arrıw-grass. Triglochin.

A rrow-head. Sagittaria sagittifolia.

Arum. Arum maculatum.

Asarabaccu. Asarum europaum.

Ash. Fraxinus excelsior.

Aspen. Populus tremula.

Asphodel. Tofieldia palustris.

Aster. Aster Tripolium.

Avens. Gellm.

Awl-wort. Subularia aquatica.

Azalea. Loiseleuria procumbens.

BaLd-MoNer. Meum Athamanticum.

Balm. Melissa officinalis.

Balsam. Impatiens.

Baneberry. Actea spicata.

Barberry. Berberis vulgaris.

Barley. Hordeum.

Barren Strawberry. Potentilla Fragariastrum.

Barrenwort. Epimedium alpinum.

Base Rocket. Reseda lutea.

13asil. Calamintha Clinopodium.

Basil Thyme. Calamintha arvensis.

Bastard Alkanet. Lithospermum arvense.

Bastard Balm. Melittis Melissophyllum.

Bastard Pimpernel. Centunculus minimus.

Bastard Toad-flax. Thesium.

Beak-sedge. Rhynchospora.

Beaked Parsley. Antkriscus vulgaris.
Bear-berry. Arctostaphylos.

Beard-grass, Polypogon.

Bedstraw. Galium.

Bee-nettle. Galeopsis speciosa.

Bee Orchis. Ophrys apifera.

Beech. Fagus sylvatica.

Beech Fern. Phegopteris polypodioides.

Beet. Bcta maritima.

Bell-flower. Campanula.

Bell-flower, Ivy-leaved. Wahlenbergia hederacea.

Belleisle-Cress. Barbarea vulgaris.

Bent-grass. Agrostis.

Bent-grass. Apera.

Berry-bearing Alder. Rhamnus Frangula.

Berry-bearing Chickweed. Cucubalus baccifer.

Betony. Stachys Betonica.

Bilberry. Vaccinium.

Bindweed. Convolvulus.

Birch. Betula.

Bird Cherry. Prunus Padus.

Bird's-foot. Ornithopus.

Bird's-foot Clover. Trigonella purpurascens.

Bird's-foot Trefoil. Lotus.

Bird's-nest. Daucus Carota.

Bird's-nest. Hypopitys multiflora.

Bird's-nest Orchis. Neottia nidus-avis.

Birthwort. Aristolochia Clematitis.

Bishop's-weed. Egopodium Podagraria.

Bishop's-weed. Ammi majus.

Bistort. Polygonum.

Bitter-Cress. Cardamine.

Bitter-sweet. Solanum Dulcamara.

Black Bent. Alopecurus agrestis.

Black Bindweed. Polygonum Convolvulus.

Black Bryony. Tamus communis.

Blackberry. Rubus, fruticosus.

Black Saltwort. Glaux maritima.

Black-thorn. Prunus communis.

Bladder-campion. Silene.

Bladder-Fern. Cystopteris.

Bladder-nut. Staphylea pinnata.

Bladder-seed. Physospermum commutatum.

Bladder-wort. Utricularia.

Blaeberry. Vaccinium Myrtillus.

Blinks. Montia fontana.

Blite. Amaranthus Blitum.

Blue-bell. Campanula rotundifolia.

Blue-bell. Scilla nutans.

Blue-bottle. Centaurea Cyanus.

Blue-eyed Grass. Sisyrinchium angusti. folium. 
Blue Sow-thistle. Lactuca alpina.

Bog Asphodel. Narthecium ossifragum.

Bog-bean. Menyanthes trifoliata.

Bog-bean, Fringed. Limnanthemum peltatum.

Bog Orchis. Liparis Loeselii.

Bog Orchis. Malaxis paludosa.

Bog Pimpernel. Anagallis tenella.

Bog-rush. Cladium germanicum.

Bog-rush. Schoenus nigricans.

Bog-rush. Scirpus.

Borage. Borago officinalis.

Box-tree. Buxus sempervirens.

Bracken. Pteris aquilina.

Brake Fern. Pteris aquilina.

Bramble. Rubus.

Brandy-bottle. Nuphar lateum.

Branks. Fagopyrum esculentum.

Briar. Rosa.

Bristle Fern. Trichomanes radicans.

Bristle-grass. Setaria.

Brooklime. Veronica Beccabunga.

Brook-weed. Samolus V'alerandi.

Brome-grass. Bromus.

Broom. Cytisus scoparius.

Broom-rape. Orobanche.

Bruise-wort. Saponaria officinalis.

Bryolly. Bryonia dioica.

Buckbean. Limnanthemum peltatum.

Buckbean. Menyanthes trifoliata.

Buckthorn. Rhamnus.

Buckwbeat. Fagopyrim.

Buckwheat. Polygonum.

Buckler Fern. Lastræa.

Bugle. Ajuga.

Bugloss. Lycopsis arvensis.

Bulrush. Scirpus.

Buirush. Typha minor.

Bullace-tree. Prunus insititia.

Bur-Marigold. Bidens.

Bur-Marigold. Xanthium strumarium.

Bur-Parsley. Caucalis.

Bur-reed. Sparganium.

Bur-weed. Medicago minima.

Bur-weed. Xanthium stmumarium.

Burdock. Arctivtm.

Burnet. Poterium.

Burnet-Saxifrage. Pimpinella.

Butcher's Broom. Ruscus aculeatus.

Butter and Eggs. Linaria vulgaris.

Butter-bur. Petasites vulgaris.

Butter-wort. Pinguicula.

Buttercup. Ranunculus bulbosus.

Butterfly Orchis. Habenaria.

Cabrage. Brassica oleracea.

Calamint. Calamintha.

Calathian Violet. Gentiana Pneumonanthe.

Campion. Iychnis.

Campion. Silene.

Canary-grass, Phalaris.
Candytuft. Iberis amara.

Caraway. Carum.

Carline Thistle. Carlina vulgaris.

Carnation. Dianthus Caryophyllus.

Carrot. Daucus Carota.

Catchfly. Lychnis.

Catchfly. Silene.

Cat-Mint. Nepeta Cataria.

Cat's-ear. Hypocheris.

Cat's-foot. Antennaria dioica.

Cat's-tail. Typha.

Cat's-tail grass. Phleum.

Celandine, Greater. 'Chelidonium majus.

Celandine, Lesser. Ranunculus Ficaria.

Celery. Apium graveolens.

Centaury, Yellow. Blackstonia perfoliata.

Centaury. Erythrea.

Chaffweed. Centunculus minimus.

Chamomile. Anthemis.

Chamomile. Matricaria Chamomilla.

Charlock. Brassica Sinapis.

Cbarlock. Raphanus Raphanistrum.

Cheese-Rennet. Galium verum.

Chequered Daffodil. Fritillaria Meleagris.

Cherry. Prunus.

Chervil. Anthriscus.

Chervil. Chorophyllum.

Chestnut. Castanea sativa.

Chickweed. Cerastinm.

Chickweed. Stellaria.

Chickweed Wintergreen. Trientalis enroper.

Chicory. Cichorium Intybus.

Chives. Allium.

Cicely. Myrrhis Odorata.

Cinquefoil. Potentilla.

Clary. Salvia.

Cleavers. Galium Aparine.

Clematis. Clematis Vitalba.

Close-reed. Dcyeuxia neglesta.

Clot-bur. Arctium majus.

Clot-bur, Spiny. Xanthium spinosum.

Cloudberry. Rubnes Chamamorus.

Clove Gilliflower. Dianthus Caryophyllus.

Clover. Tritolium.

Clover, Yellow. Medicago lupulina.

Club-moss. Lycopodium.

Club-rush. Eleocharis.

Club-rush. Scirpus.

Cob-nut. Corylus Avellana.

Cock's-foot grass. Dactylis glomerata.

Cock's-head. Onobrychis.

Codlins-and-Cream. Fpilobium hirsutum.

Cole-seed. Brassica Napus.

Colt's-foot. Petasites.

Colt's-foot. Tussilago.

Columbine. Aquilegia vulgaris.

Colza. Brassica Rutabaga.

Comfrey. Symphytum.

Connemara Heath. Dabocia polifolia.

Coral-root. Corallorhiza innata. 
Coral-wort, Cardamine bulbifera.

Cord-grass. Spartina.

Coriander. Coriandrum sativum.

Corn Cockle. Lychnis Githago.

Corn Bell-flower. Specularia hybrida.

Corn-flower. Centaurea Cyamus.

Corn Marigold. Chrysanthemun segetum.

Corn Parsley. Carim segetum.

Corn Rose. Papaver Rhaens.

Corn-salad, Valerianella.

Corn Spurrey. Spergula arvensis.

Cornel. Cornus.

Cornish Lovage. Physospermum commutatum.

Cornish Money-wort. Sibthorpia eltropaa.

Cotton-grass. Eriophorum.

Cotton 'Thistle. Onopordon Acrnthium.

Cotton-weed. Diotis maritima.

Couch-grass. Agropyron.

Cowbane. Cicuta virasa.

Cowberry. Vaccinium Vitis-Idaa.

Cow-cress. Lepidium campestre.

Cow-grass. Trifolium medium.

Cow-herb. Saponaria Vaccaria.

Cow-Parsley. Anthriscus sylvestris.

Cow-Parsnip. Heraclenm Sphondylium.

Cowslip. Primula veris.

Cow-wheat. Melumpyrum.

Crab-tree. Pyrus Malus.

Crakeberry. Empctrum nigrum.

Cranberiy. Schollera Oxycoccus.

Crane's-bill. Goranium.

Creeping Jenny. Lysimachia Nummularia.

Cress. Lepidium.

Cress Rocket. Vella annua.

Crested Hair-grass. Koeleria cristata.

Crocus. Crocus.

Crosswort. Galium Cruciata.

Crowberry. Empetrum nigrum.

Crowfoot. Ramineulus.

Crown Vetch. Coronilla varia.

Cuckoo-flower. Cardamine pratensis.

Cuckoo-pint. Arum.

Cudweed. Filago.

Cudweed. Gnaphalium.

Currant. Ribes.

Cushion-Pink. Silenc acaulis.

Cut-grass. Leersia oryzoides.

Cyclamen. Cyclamen hederafolium.

Cyphel. Arenaria sedoides.

Cypripede. Cypripedium Calceolus.

DAFFodIL. Narcissus.

Daffa-down-dilly. Narcissus Pseudo-Narcissus.

Daisy. Bellis perennis.

Dame's Violet. Hesperis matronalis.

Dandelion. Taraxircum officinale.

Danewort. Sambucus Fibulus.

Darnel. Lolium.

Dead-nettle. Lamium.
Deadly Night-shade. Atropa Belladonna.

Deer Fern. Lomaria Spicant.

Dewberry. Rubus casius.

Ditch Reed. Phragmites communis.

Dittander. Lepidium latifolium.

Dock. Rumex.

Dodder. Cuscuta.

Dogberry. Cornus sanguinea.

Dog-grass. Agropyron repens.

Dog-rose. Rosa canina.

Dog's-tail grass. Cynosurus.

Dug's-tooth grass. Fibichia umbellata.

Dog-violet. Viola.

Dogwood. Cornus sanguinea.

Dove's-foot. Geranium dissectum.

Drone Orchis. Ophrys aranifera, $v$. fucifera.

Dropwort. Spiraa.

Duckweed. Lemna.

Duckweed. Wolffia arrhiza.

Dutch Clover. Trifolium repens.

Dutch Rush. Equisetum hyemale.

Dwale. Atropa Belladonna.

Dyer's Greenweed. Genista tinctoria.

Dyer's Rocket. Reseda Lnteola.

Dyer's Woad. Isatis tinctoria.

Earth-xut. Conopodium denudatum.

Earth-nut. Carum Bulbocastanum.

Earth-smoke. Fumaria. officinalis.

Eglantine. Rosa rubiginosa.

Elder. Sambucus.

Elecampane. Inula Helenium.

Elm. Ulmus.

Enchanter's Nightshade. Circea.

Endive. Cichornum Intybus.

Eryngo. Erynainm.

Evening Primrose. Enothera.

Everlasting. Antennaria.

Everlasting Pea. Lathyrus.

Eye-bright. Euphrasia officinalis.

False Broye-grass. Drachypodium.

False Oat. Arrhenatherum avenaceum.

Fat Hen. Chenopodium albusn.

Featherfoil. Hottonia palustris.

Feather-grass. Stipa pennata.

Felwort. Gentiana Amarella.

Fennel. Foniculum officinale.

Fen Orchis. Liparis Loeselii.

Fen Sedge. Cludium germanicum.

Fenugreek. Trigonella purpurascens.

Fescue-grass. Fistuca.

Feverfew. Chrysanthemum Parthenium.

Feverfew. Matricaria inodora.

Field Cabbage. Brassica Rutabaga.

Field Marlder. Sherardia arvensis.

Fiddle Dock. Rumex pulcher.

Figwort. Scrophnlaria.

Filbert. Corylus Avellana.

Filmy Fern. Hymenophyllum. 
Finger-grass. Panicum.

Fiorin-grass. Agrostis palustris, v. sto. lonifera.

Flag. Iris.

Flax. Linum.

Flax-seed. Radiola linoides.

Flea-bane. Erigeron.

Flea-bane. Inula.

Flea-bane. Pulicaria.

Flea-wort. Senecio.

Fleur-de-luce. Iris Psend-acorus.

Flix-weed. Sisymbrium Sophia.

Flote-grass. Glyceria fuitans.

Flowering Ferv. Osmunda regalis.

Flowering Rush. Butomus umbellatus.

Fluellin. Linaria.

Fly Orchis. Ophrys muscifera.

Fool's Parsley. Ethusa Cynapinm.

Fool's Water-cress. Apium nodiflorum.

Forget-me-not. Myosotis.

Fox-glove. Digitalis purpurea.

Fox-tail grass. Alopecurus.

Fragrant Orchis. Habenaria conopsea.

Fritillary. Fritillaria Meleagris.

Frog-bit. Hydrocharis Morsus-rane.

Frng Orchis. Habenaria viridis.

Fuller's herb. Saponaria officinalis.

Fumitory. Corydalis.

Fumitory. Fumaria.

Furze. Ulex.

Gale. Myrica Gale.

Galingale. Cyperus longus.

Garlic. Allium.

Gean-tree. Prumus _4vium.

Gentian. Gentiana.

Gentianella. Microcala filiformis.

Geranium. Geranium.

Germander. Teucrium.

Gilliflower. Cheiranthus Cheiri.

Gilliflower. Mathiola incana.

Gipsy-flower. Cynoglossum officinale.

Gipsy-wort. Lycopus europaus.

Glasswort. Salicornia.

Globe-flower. Trollius europaus.

Goat-weed. Agopodium Podagraria.

Goat's-beard. Tragopogon.

Gold-of-Pleasure. Camelina.

Golden-locks. Astcr Linosyris.

Golden Moss. Sedum acre.

Golden-rod. Solidago Virgaurea.

Golden Samphire. Inula crithmoides.

Golden Saxifrage. Chrysosplenium.

Goldilocks. Ranunculus auricomus.

Good King Henry. Chenopodium Bonus-

Henricus.

Gooseberry. Ribes Grossularia.

Goose-corn. Juncus squarrosus.

Goose-foot. Chenopodium.

Gone-foot. Sucda.

Goose-grass. Galium Aparine.
Goose-tongue. Achillea Ptarmica.

Goose-weed. Potentilla Anserina.

Gorse. Ulex europaus.

Goutweed. Egopodium podagraria.

Grape Hyacinth. Muscari racemosum.

Grass of Parnassus. Parnassia palustris.

Grass Poly. Lythrum Hyssopifolia.

Grass Wrack. Zostera.

Greek Valerian. Polemonium coruleum.

Green-Weed. Genista.

Grey Mill. Lithospermum officinale.

Gromwell. Lithospermum.

Gromwell, Sea. Mertensia maritima.

Ground-Ivy. Nepeta Glechoma.

Ground-Pine. Ajuga Chamapitys.

Groundsel. Senecio.

Guelder-Rose. Viburnum.

Guimaure. Althea officinalis.

HaIr-grass. Aira.

Hair-grass. Deschampsia.

Hair-grass, Grey. Corynephorus canescens.

Hard Fern. Lomaria Spicant.

Hard-grass. Lepturus filiformis.

Hare-bell. Campanula rotundifolia.

Hare's-ear. Bupleurum.

Hare's-tail grass. Lagurus ovatus.

Hart's-tongue. Scolopendrium vulgare.

Hartwort. Tordylium.

Hassocks. Deschampsia cœspitosa.

Haver. Avena fatua.

Hawkbit. Leontodon.

Hawkweed. Hieracium.

Hawk's-beard. Crepis.

Hawthorn. Crategus Oxyacantha.

Hay-scented Fern. Lastraa amula.

Hazel. Corylus Avellana.

Heart's-ease. Viola tricolor.

Heath. Erica.

Heath-grass. Sicglingia decumbens.

Heath-pea. Lathyrus macrorrhizus.

Heather. Calluna Erica.

Hedge-Garlic. Sisymbrium Alliaria.

Hedge-Mustard. Erysimum.

Hedge-Mustard. Sisymbrium.

Hedge-Parsley. Caucalis.

Hellebore. Helleborus.

Helleborine. Cephalanthera.

Helleborine. Epipactis.

Helminth. Picris echioides.

Hemluck. Coninm maculatum.

Hemp. Cannabis sativa.

Hemp-Agrimony. Eupatorium cannabinum.

Hemp-nettle. Galeopsis.

Henbane. Hyoscyamus niger.

Henbit. Lamium amplexicaule.

Hen's-foot. Caucalis.

Herb Bennet. Geum urbanum.

Herb Christopher. Actea spicata.

Herb Gerard. Agopodium Podagraria.

Herb Gerard. Peucedanum Ostruthium. 
Herb Paris. Paris quadrifolia.

Herb Robert. Geranium Robertianum.

Herb St. Barbara. Barbarea vulgaris.

Herb Twopence. Lysimachia Nimmularia.

Heron's Bill. Erodium cicutariurn.

High-taper. Verbascum Thapsus.

Hoary Cress. Lepidium Draba.

Hog's Fennel. Peucedanum.

Hogweed. Heracleum Sphondylium.

Holly. Ilex Aquifolium.

Holly Fern. Polystichum Lonchitis.

Holy-grass. Hierochloe borealis.

Honewort. Sison Anomum.

Honewort. Trinia vulgaris.

Honeystalks, Trifolium pratense.

Honeysuckle. Lonicera.

Hooded Milfoil. Utricularia vulgaris.

Hop. Humulus Lnopulus.

Horehound. Ballota.

Horehound. Marrubinm vulgare.

Horehound, Water. Lycopus europaus.

Hornbeam. Carpinus Betulus.

Horned Poppy. Glaucium.

Horned Poppy, Violet. Rameria hybrida.

Horned Pundweed. Zannichellia.

Hornwort. Ceratophyllum.

Horse Radish. Cochlearia Armoracia.

Horseshoe Vetch. Hippocrepis comosa.

Horsetail. Equisetum.

Hound's-tongue. Cynoglassum.

House-leek. Sempervivum tectorum.

Hyacinth. Scilla nutans.

IRIs. Iris.

Isle-of-Man Cabbage. Brassica monensis. Ivy. Hedera Helix.

Ixia. Romulea Columna.

JACK-BY-THE-HedGe. Sisymbrium Alliaria. Jacob's Ladder. Polemonium caruleum. Jagged Chickweed. Holosteum umbellatum. Jerusalem Cowslip. Pulmonaria officinalis. Joint Vetch. Ornithopus ebracteatus.

Juniper. Juniperus.

KALE. Brassica oleracea.

Kedlock. Brassica Sinapis.

Kidney Vetch. Anthyllis Vulneraria.

Kingcup. Ranunculus acris.

Kipper-nut. Conopodium denudatum.

linapweed. Centaurca.

Knawel. Scleranthus.

Knot-grass. Illecebrum verticillatum.

Kinot-grass. Polygonum.

Ladies' Fixeer. Anthyllis Vulneraria.

Lady Fern. Athyrium.

Lady's Cushion. Saxifraga hypnoides.

Lady's Bed-straw. Galium.

Iady's Mantle. Alchemilla.

Lady's Slipper. Cypripedium Calceolus.
Lady's Smock. Cardamine.

Lady's Tresses. Goodyera repens.

Lady's Tresses. Spiranthes.

Lamb's Toe. Anthyllis vulneraria.

Lamb's Lettuce. Valerianella.

Lamb's Succory. Amoseris pusilla.

Lamb's Tongue. . Plantago media.

Larkspur. Delphinium.

Lavender Thrift. Statice Limonium.

Leek. Allium.

Lent Lily. Narcissus Pseudo-Narcissus.

Leopard's-bane. Doronicum.

Lettuce. Lactuca.

Limestone Fern. Phegopteris Robertiana.

Lily. Lilium.

Lily of the Valley. Convallaria majalis.

Lime Tree. Tilia.

Linden Tree. Tilia vulgaris.

Ling. Calluna Erica.

Linnæa. Linncea borealis.

Liquorice. Ononis repens.

Liquorice Vetch. Astragahus glycyphyllos.

Live-long. Sedum Telephium.

Lizard Orchis. Orchis hircinum.

London Pride. Saxifraga.

London Rocket. Sisymbrizm Irio.

Loose-strife. Lysimachia.

Loose-strife. Lythrum.

Lords and Ladies. Arum maculatum.

Lousewort. Pedicularis.

Lovage. Ligusticum scoticum.

Lucerne. Medicago.

Lungwort. Pulmonaria.

Lyme-grass. E'ymus.

Lyme-grass. Hordenm sylvaticum.

Madder. Rubia peregrina.

Madderwort. Asperugo procumbens.

Madwort. Alyssum.

Maiden-hair. Adiantum Capillus-Veneris.

Maiden-hair. Briza media.

Maiden-bair. Gymnogramme leptophylla.

Male-fern. Lastrca Filix-mas.

Mallow. Malva.

Man Orchis. Aceras anthropophora.

Man Tway-blade. Aceras anthropophora.

Manna Grass. Glyceria fluitans.

Maple. Acer.

Mare s-tail. Hippuris vulgaris.

Marjoram. Origanum vulqare.

Marl Grass. Trifolium pratense.

Marram. Ammophila.

Marsh Centaury. Microcala filiformis.

Marsh Cistus. Ledum palustre.

Marsh Felwort. Swertia perennis.

Marsh Fern. Lastrcea Thelypteris.

Marsh Mallow. Althea.

Marsh Marigold. Caltha palustris.

Marsh Parsley. Selinum Carvifolia.

Marsh Samphire. Salicornia.

Marsh Trefuil. Menyanthes trifoliata. 
Marsh Tway-blade. Malaxis paludosa.

Marsh-wort. Apium nodiflorum.

Martagon Lily. Lilium.

Master-wort. Peucedanum Ostruthium.

Mat-grass. Nardus stricta.

May. Crategus Oxyacantha.

May-flower. Cardainine pratensis.

May Lily. Maianthemum Convallaria.

Mayweed. Anthemis Cotula.

Mayweed. Matricaria inodora.

Maywort. Galium Cruciata.

Meadow-grass. Glyceria.

Meadow-grass. Poa.

Meadow-bout. Caltha palustris.

Meadow Pink. Lychnis Flos-cuculi.

Meadow Rue. Thalictrum.

Meadow-sweet. Spircea Ulmaria.

Meal-tree. Viburnum Lantana.

Medick. Medicago.

Medlar. Cotoneaster.

Medlar. Pyrus germanica.

Melic-grass. Melica.

Melic-grass, Blue. Molinia carulea.

Melilot. Melilotus.

Menziesia. Dabocia polifolia.

Menziesia. Phyllodoce taxifolia.

Mercury. Mercurialis.

Meu. Meum Athamanticum.

Mezereon. Daphne Mezereum.

Michaelmas Daisy. Aster Tripolium.

Mignonette. Reseda.

Milfoil. Achillea Millefolium.

Milk Parsley. Peucedanum palustre.

Milk-thistle. Silybum Marianium.

Milk-vetch. Astragalus.

Milk-vetch. Oxytropus.

Milk-wort. Polygala.

Mill Mountain. Linum catharticum.

Millet-grass. Milium effusum.

Mint. Mentha.

Mistletoe. Viscum album.

Mithridate Mustard. Thlaspi arvense.

Moneywort. Lysimachia Nummuluria.

Monkey-flower. Mimulus luteus.

Monkey Orchis. Orchis Simia.

Monk's-hood. Aconitum Napellus.

Mouk's Rhubarb. Rumex alpinus.

Moon Daisy. Chrysanthemum Leucan. themun.

Moonwort. Botrychium Lunaria.

Moor-grass. Sesleria cerndea.

Moschatel. Adoxa Moschatellina.

Motberwort. Leonurus Cardiaca.

Mountain Asb. Pyrus Aucuparia.

Mountain Avens. Dryas octopetala.

Mountain Sorrel. Oxyria digyna.

Mountain Stone-parsley. Seseli Libanotis.

Mouse-ear Chickweed. Cerastium.

Mouse-ear Chickweed. Holosteum umbellatum.

Mouse-tail. Myosurus minimus.
Mudwort. Limosella aquatica.

Mugwort. Artemisia.

Mugwort. Galium Cruciata.

Mullein. Verbascum.

Musk Orchis. Herminium Monorchis.

Mustard. Brassica.

Myrtle. Myrica Gale.

Myrtle-flag. Acorus Calamus.

NAILwort. Erophila vulgaris.

Navel-wort. Cotylcdon Ümbilicus.

Navew. Brassica Rutabaga.

Needle Whin. Genista anglica.

Nettle. Urtica.

Nightshade. Solanum.

Nipplewort. Lapsana communis.

Nipplewort, Dwarf. Arnoseris pusilla.

Nit-grass. Gastridium.

None-so-pretty. Saxifraga umbrosa.

Nonsuch. Medicago iupulina.

OAk. Quercus.

Oak Fern. Phegopteris Dryopteris.

Oat-grass. Avena.

Old-man's Beard. Clematis Vitalba.

One-berry. Paris quadrifolia.

Orache. Atriplex.

Orchis. Habenaria.

Orchis. Ophrys.

Orchis. Orchis.

Orpine. Sedum.

Osier. Salix.

Ox-eye. Chrysanthemum.

Oxlip. Primula elatior.

Ox-tongue. Picris.

Oyster-plant. Mertensia maritima.

Paonr. Paenia corallina.

Paigle. Primula veris.

Painted Cup. Bartsia.

Panic-grass. Panioum Crus-galli.

Panic-grass. Setaria.

Pansy. Viola.

Parsley. Carum.

Parsley-fern. Cryptogramma crispa.

Parsley-Piert. Alchcmilla arvensis.

Parsnip. Peucedanum sativum.

Pasque-flower. Anemone Pulsatilla.

Pea. Lathyrus.

Pear. Pyrus communis.

Pearl-wort. Sagina.

Pellitory. Parietaria officinalis.

Penny-cress. Thlaspi.

Penny-royal. Mentha Pulegium.

Pennywort. Hydrocotyle vulgaris.

Peppermint. Mentha Pipcrita.

Pepper-grass. Pilularia globulifera.

Pepper Saxifrage. Silaus pratensis.

Pepperwort. Lepidium.

Periwinkle. Vinca.

Persicaria. Polygonum. 
Petty Whin. Genista anglica.

Pheasant's eye. Adonis autumnalis.

Pignut. Conopodium denudatum.

Pile-wort. Ranunculus Ficaria.

Pill-wort. Pilularia globulifera.

Pimpernel. Anagallis.

Pimpernel, Yellow. Lysimachia nemorum.

Pine. Pinus.

Pink. Dianthus.

Pipewort. Eriocaulon septangulare.

Plane, Scotcb. Acer pseudo-platanus.

Plantain. Plantago.

Ploughman's Spikenard. Inula Conyza.

Plum. Prunus domestica.

Polypody. Polypodium.

Pond-weed. Potamogeton.

- Poor-man's Parmacetic. Capsella Bursapastoris.

Poor-man's Pepper. Lepidium latifolium.

Poor-man's Rhubarb. Thalictrum alpinum.

Poor-man's Weather-glass. Anagallis arvensis.

Poplar. Populus.

Poppy. Papaver.

Prickly-Samphire. Echinophora spinosa.

Prickwood. Cornus sanguinea.

Primrose. Primula.

Primrose Peerless. Narcissus biflorus.

I'rivet. Ligustrum vulgare.

Purslane, Sea. Arcnaria peploides.

Purslane. Peplis Portula.

QUAKING-Ǵass, Briza.

Queen-of-the-meadows. Spirca Ulmaria.

Quill-wort. Isoetes.

RADISH. Raphanus.

Ragged Robin. Lychnis Flos-cuculi.

Ragwort. Senecio.

Rampion. Phyteuma.

Ramsons, Allizm.

Rape. Brassica Napus.

Raspberry. Rubus Idous.

Red Eyc-bright. Bartsia Odontites.

Red Rattle. Pedicularis.

Red-rot. Drosera rotundifolia.

Red-shanks. Tillea muscosa.

Reed. Phragmites communis.

Reed-mace. Typha.

Rest-harrow. Ononis.

Ribbon-grass. Phalaris armindinacea.

Ribgrass. Plantago lanceolata.

Roast-beef-plant. Iris fotidissima.

Rock-brakes. Cryptogramma crispa.

Rockcist. Helianthemum Chamøeistus.

Rock-cress. Arabis.

Rock-rose. Helianthemum.

Rocket. Diplotaxis.

Roebuck-berry. Rubus Chamomorus.

Roebuck-berry. Rubus saxatilis.

Rose. Rosa.
Rose-bay. Epilobium angustifolium.

Rose Campion. Lychnis diurna.

Rosemary. Andromeda polifolia.

Rose of Heaven. Lychnis Floscuculi.

Rose-root. Sedum Rhodiola.

Rowan-tree. Pyrus Aucuparia.

Royal Fern. Osnunda regalis.

Runch. Brassica Sinapis.

Rupture-wort. Herniaria.

Rush. Juncus.

Rusty-back. Ceterach officinarum.

Rye-grass. Lolium.

Safrron. Colchicum autumnale.

Sage. Salvia.

Saintfoin. Onobrychis sativa.

Salad Burnet. Poterium.

Sallow. Salix.

Sallow-thorn. Hippophaë rhamnoides.

Salsify. Tragopogon porrifolius.

Saltwort, Black. Glaux maritima.

Saltwort, Prickly. Salsola Kali.

Saltwort, Sbrubby. Sucda fruticosa.

Samphire. Crithmum maritimum.

Sand Chickweed. Sagina nodosa.

Sand-grass. Mibora verna.

Sand Rocket. Diplotaxis muralis.

Sandwort. Alsine.

Sandwort. Arenaria.

Sandwort-Spurrey. Lepigonum.

Sanicle. Sanicula europea.

Sauce-alone. Sisymbrium Alliaria.

Saw-wort. Serratula tinctoria.

Saw-wort, Alpine. Saussurea alpina.

Saxifrage. Saxifraga.

Saxifrage. Sescli Libanotis.

Scabious. Scabiosa.

Scale Fern, Ceterach officinarum.

Scorpion-grass. Myosatis.

Seotch Fir. Pinus sylvestris.

Scotch 'Thistle. Onopordon Acanthium.

Scull-cap. Scutellaria.

Scurvy-grass. Cochlearia.

Sea Bent. Ammophila arnndinacea.

Sea Blite. Suceda.

Sea Buckthorn. Hippophä̈ rhamnoides.

Sea Bugloss. Mertensia maritima.

Sea Chickweed. Arenaria peploides.

Sea Gilliflower. Armeria maritima.

Sea-grass. Zostera marina.

Sea Heath. Frankenia.

Sea Holly. Erynginm maritimum.

Sea Kiale. Crambe maritima.

Sea Lavender. Statice.

Sea Matweed. Ammophila arundinacea.

Sea Milkwort. Glaux maritima.

Sea Parsnip. Echinophora spinosa.

Sea Pimpernel. Arenaria peploides.

Sea Pink. Armeria maritima.

Sea Poppy. Glaucium flavum.

Sea Purslame. Arenaria peploides. 
Sea Purslame. Atriplex portulacoides.

Sea Reed. Ammophila arundinacea.

Sea Rocket. Cakile maritima.

Sea Starwort. Aster Tripolium.

Sea Stock. Mathiola sinuata.

Sea Sulphurwort. Peucedanum officinale.

Sea Tassel-grass. Ruppia spiralis.

Sedge. Carex.

Selt-heal. Prunella vulgaris.

Service-tree. Pyrus domestica.

Service-tree. Pymus torminalis.

Setter-wort. Helleborus foetidus.

Shamrock. Medicago lupulina.

Shamrock. Oxalis acetosella.

Shave-grass. Equisetum hyemale.

Sheep's-bit. Jasione montana.

Sheep's Scabious. Jasione montana.

Sheep's Sorrel. Rumex acetosella.

Shepherd's-needle. Scandix Pecten-Veneris.

Shepherd's-purse. Capsclla Bursa-Pastoris.

Shepherd's Staff. Dipsacus pilosus.

Shepherd's Weather-glass. Anagallis arvensis.

Shield Fern. Lastrcea.

Shield Fern. Polystichum.

Shore-grass. Littorella.

Silver-weed. Potentilla Anserina.

Skull-cap. Scutellaria.

Sloe. Prunus communis.

Slough-heal. Prunella vulgaris.

Small-reed. Calamagrostis.

Small-reed. Deyeruxia.

Smallage. Apium graveolens.

Smith's Fern. Phegopteris polypodioides.

Snake-weed. Polygonum Bistorta.

Snake's head. Fritillaria Meleagris.

Snapdragon. Antirrhinum.

Snapdragon. Linaria Cymbalaria.

Sneeze-wort Yarrow. Achillea Ptarmica.

Snowdrop. Galanthus nivalis.

Snowfiake. Leucojum.

Soap-wort. Saponaria.

Soft-grass. Holcus.

Solomon's Seal. Polygonatum.

Sorrel. Rumex.

Sorrel, Mountain. Oxyria digyna.

Sorrel, Wood. Oxalis Acetosella.

Southernwood. Artemisia campestris.

Sow-bread. Cyclamen hedercefolium.

Sow-thistle. Sonchus.

Spearwort. Ranunculus

Spearmint. Mentha viridis.

Speedwell. Veronica.

Spider Orchis. Ophrys aranifera.

Spiderwort. Lloydia alpina.

Spignel. Meum Athamanticum.

Spike-rush. Eleocharis palustris.

Spinach. Chenopodium Bonus-Henricus.

Spindle-tree. Euonymus europaus.

Spleenwort. Asplenium.

Spring-grass. Anthoxanthum odoratum.
Spurge. Euphorbia.

Spurge Laurel. Daphne Laureola.

Spurge Olive. Daphne Vezereum.

Spurrey. Sagina nodosa.

Spurwort. Sherardia arvensis.

Squats. Glancium flavum.

Squill. Scilla.

Squinancy-wort. Asperula cynanchica.

Squirrel-tail grass. Hordeum maritimum.

St.Barnaby's Thistle. Centaureasolstitialis.

St. Dabeoc's Heath. Dabeocia polifolia.

St. James' weed. Capsella Bursa-Pastoris.

St. John's wort. Hypericum.

St. Patrick's Cabbage. Saxifraga umbrosa.

St. Peter's wort. Hypericum quadratum.

Star of Bethlehem, Yellow. Gagea fascicularis.

Star of Bethlehem. Ornithogalum.

Star-of-the-Farth. Plantago Coronopus.

Star-fruit. Damasonium stellatum.

Star-thistle. Centaurea.

Star-wort. Stellaria.

Starch Hyacintl.. Muscari racemosum.

Stickseed. Echinospermum Lappula.

Stitchwort. Stellaria.

Stock. Mathiola.

Stone Bramble. Rubus saxatilis.

Stone-crop. Sedum.

Stone Parsley. Sison Amomum.

Stone-wort. Chara.

Stone-wort. Sison Amomum.

Stone-wort. Trinia vulgaris.

Stork's-bill. Erodium.

Strapwort. Corrigiola littoralis.

Strawberry. H'ragaria.

Strawberry, Barren. Potentilla Fragariastrum.

Strawberry-tree. Arbutus Unedo.

Succory. Cichorium Intybus.

Succory, Yellow. Picris hieracioides.

Sulphur-wort. Silaus pratensis.

Sundew. Drosera.

Sun Cistus. Helianthemum polifolium.

Sun Spurge. Euphorbia Hetioscopia.

Sun-rose. Helianthemum.

Swallow-wort. Chelidonium majus.

Swedish Turnip. Brassica Rutabaga.

Sweetbriar. Rosa mubiginosa.

Sweet-flag. Acorus Calamus.

Sweet-grass. Glyceria.

Sweet Sedge. Acorus Calamus.

Swine's Cress. Senebiera.

Swine's Succory. Arnoseris pusilla.

Sword-flag. Gladiolus illyricus.

Sycamore. Acer Pseudo-platanus.

TAMarisk. Tamarix gallica.

Tansy. Tanacetum vulgare.

Tare. Vicia.

Tassel Pondweed. Ruppia spiralis.

Tea plant. Lycium barbarum. 
Teasel. Dipsacus.

Thale-cress. Sisymbrium Thatianum.

Thistle. Cardurus.

Thistle. Cnicus.

Thorn-Apple. Datura Stramonium.

Thorougl1-wax. Bupleurum rotundifolium.

Thrift. Armeria.

Thrum-wort. Damasonium stellatum.

Thyme. Thymus.

'Timothy-grass. Phleum.

Toad-flax. Linaria.

Toad Rush. Juncus bufonius.

Tongue-under-tongue. Astragalus Hypoglottis.

Tooth-wort. Lathrea squamaria.

' Tooth-cress. Cardamine bulbifera.

Tormentil. Potentilla.

Touch-me-not. Impatiens Noli-me-tangere.

Tower Mustard. Arabis perfoliata.

'Tower Turkey-pod. Arabis Turrita.

Traveller's Joy. Clematis Vitalba.

Treacle Mustard. Erysimum cheiranthoides.

Tree Mallow. Lavatera.

Trefoil. Trifolium.

Trembling-grass. Briza.

True Love. Paris quadrifolia.

Tulip. Tulipa sylvestris.

Turkey Pod. Sisymbrium Thalianum.

Turk's-Cap Lily. Lilium Martagon.

Turnip. Brassica Rapa.

Tutsan. Hypericum Androscmum.

Tway-blade. Listera.

Twig-rush. Cladium gormanicum.

Valenias. Centranthus.

Valerian. Valeriona.

Venus' Comb. Scandix Pecten-Veneris.

Venus' Looking-glass. Specularia hybrida.

Vernal-grass. Anthoxanthum.

Vervain. Terbena officinalis.

Vetch. Lathyrus.

Vetch. Vicia.

Vetchling. Lathyrus.

Violet. Viola.

Viper-grass. Echium vulgare.

Viper's-bugloss. Echium.

Virginian Stock. Malcolmia maritima.

Wake RoBix. Arum maculatum.

Wall-cress. Arabis.

Wallflower Cabbage. Brassica.

Wallflower. Cheiranthus Cheiri.

Wallflower Mustard. Erysimum cheiranthoides.

Wall Mustard. Diplotaxis.

Wall Pennywort. Cotyledon Umbilicus.

Wall Pepper. Sedum acre.

Wall Rue. Asplenium Rutu-muraria.

Wart-cress. Senebicra.

Wart-wort. Enphorbia Helioscopin.

Water Aloe. Stratiotes aloides.
Water Avens. Geum rivale.

Water Betony. Scrophularia.

Water Caltrops. Caltha palustris.

Water-can. Nuphar luteun.

Water Cbickweed. Montia fontana.

Water-cress. Nasturtium.

Water Dropwurt. Enanthe.

Wuter Fennel. Ranunculus trichophyllus.

Water Hair-grass. Catabrosa aquatica.

Water Hemlock. Cicuta virosa.

Water Horse-tail. Chara.

Water Horse-tail. Nitella.

Water Lily, White. Nymphoea alba.

Water Lily, Yellow. Nuphar.

Water Lily, Fringed. Limnanthemum peltatum.

Water Milfoil. Myriophyllum.

Water Milfoil. Utricularia.

Water Parsnip. Apizom.

Water Parsnip. Sium.

Water Pepper. Elatine Hydropiper.

Water Pepper. Polygonum Hydropiper.

Water Pimpernel. Samolus I alerandi.

Water Plantain. Elisma natans.

Water Radish. Nasturtium.

Water Rocket. Nasturtium.

Water Soldier. Stratiotes aloides.

Water Starwort. Callitrichc.

Water Thyme. Elodea canadensis.

Water Violet. Hottonia palustris.

Water-weed. Elod a canadensis.

Water-wort. Elatine.

Way Bennet. Horderm murinum.

Way-bread. Plantago major.

Wayfaring tree. Tiburnum Lantana.

Weasel-snout. Lamium Galeobdolon.

Welcl. Reseda Luteola.

Welsh Poppy. Mecanopsis cambrica.

Wheat-grass. Agropyron.

Whin. Ulex curopeus.

Whip-tongue. Galium Mollugo.

White-beam. Pyrus.

White Bottle. Silenc Cucubalus.

White Thorn. Cratagus.

Whitlow-grass. Draba.

Whitlow-grass. Erophila.

Whorl-grass, Catabrosa aquatica.

Whortle-berry. Vaccinium.

Wild Williams. Lychnis Flos-cuculi.

Willow. Salix.

Willow-lıerb. Epilobium.

Wind-flower. Anemone nemorosa.

Wind-grass. Apera interrupta.

Winter Aconite. Eranthis hyemalis.

Winter-cress, Barbarea.

Winter-green, Moneses grandiflora.

Winter-green. Pyrola.

Winter-green, Clickweed. Trientalis europace.

Winter Helintrope. Petasites fragrans.

Withy. Salix fragilis. 
Woad. Genista tinctoria.

Woad. Reseda Luteola.

Woak-waxen. Genista tinctoria.

Wolf's bane. Aconitum Napellus.

Woodbine. Lonicera.

Woodruff. Asperula.

Wood-rush. Luzula.

Wood Sage. Teucrium Scorodonia.

Wood Sorrel. Oxalis.

Worm-seed. Erysimum.

Wormwood, Artemisia.

Woundwort. Anthyllis Vulneraria.

Woundwort. Stachys.
Wrack-grass. Zostera.

Wych Elm. Ulmus montana.

Wych Hazel. Ulmus montana.

YARR. Spergula arvensis.

Yarrow. Achillea.

Yellow-cress. Nasturtium.

Yellow Rattle. Rhinanthus Crista-galli.

Yellow Rocket. Barbarea.

Yellow-weed. Reseda Luteola.

Yellow-wort. Blackstonia perfoliata.

Yew. Taxus baccata.

Yorkshire Fog. Holcus lanatus. 


\section{BOOKS FOR BOTANISTS.}

LONDON CATALOGUE OF BRITISH PLANTS. Part I., containing the British Phrnogamia, Filices, Equisetacex, Lycopodiacex, Selaginellacex, Marsileacex, and Characeæ. Eighth Edition. Demy 8vo. 6d.; on stouter paper, in limp cloth, Is. Generic Index only, on card, $2 d$.

SOWERBY'S ENGLISH BOTANY: Containing a Description and Life-size coloured Drawing of every British Plant. Edited and brought up to the Present Standard of Scientific Knowledge by T. Boswell (formerly SyME), LL.D., F.L.S., \&c. For the Contents, \&c., see next page.

BOTANICAL LABELS, for labelling Herbaria, adapted to the names in the London Catalogue and the Manuals of Babington and Hooker, with extra labels for new varieties. By JoHn E. RoBson. Demy 8vo. 5 s.

THE BOTANIST'S POCKET BOOK. By W. R. HAYWARD. Containing, arranged in a tabulated form, the chief characteristics of British Plants. Fcap. 8vo, flexible binding for the pocket, revised, with new Appendix. Sixth Edition, 4s. 6d.

"The diagnoses seem framed with considerable care and judgment, the characteristics having been well selected and contrasted."-Journal of Botany.

BRITISH MOSSES: their Homes, Aspects, Structure and Uses. Containing a Coloured Figure of each species, etched from Nature. By F. E. TRIPP. Illustrated with 39 beantifully Coloured Plates. New and Revised Edition. Two volumes, $£ 2$ I $2 s .6 d$. Offered at $£$ I I 5 s.

RAMBLES IN SEARCH OF WILD FLOWERS, and How to Distinguish them. By Margaret Plues, Author of "Rambles in Search of Flowerless Plants," "British Grasses," \&c. With 96 coloured figures and numerous cuts. Third Edition, large post $8 \mathrm{vo}, 7 s .6 d$.

"This little book has already, we are pleased to note, attained its third edition. We sincerely wish it that continuance of public favour which it so well deserves."Saturday Review.

THE FLORA OF DERBYSHIRE. By the Rev. W. H. PAINTER, Corresponding Member of the Birmingham Natural History and Microscopical Society. Giving a brief Description of the Physical Features of Derbyshire, and a List of all the Flowering Plants and Ferns found therein, with their principal Habitats. Demy 8vo. with Map, price $7 s .6 d$.

By J. G. BAKER, F.R.S., F.L.S., First Assistant in the Herbarium of the Royal Gardens, Kew.

HANDBOOK OF THE AMARYLLIDEÆ, including the Alstrameries and Agaveæ. Demy 8vo. 5 s.

"It is impossible to speak highly enough of a work of this kind, entailing labours of half a lifetime; the least of those who are interested in the subject can do is to buy and study it."-Gardeners' Chronicle.

HANDBOOK OF THE FERN ALLIES. A Synopsis of the Genera and Species of the Natural Orders Equisetaceæ, Lycopodiacex, Selaginellaceæ, Rhizocarpeæ. Demy 8vo. $5^{s}$.

"Mr. Baker's intimate acquaintance with the class of plants of which he treats is sufficient guarantee of the excellence of the work. It fills a gap which has long been felt."-Academy.

\section{A FLORA OF THE ENGLISH LAKE DISTRICT. Demy 8vo. $7 s .6 d$. \\ "A model of what a local flora should be."-Guardian. Just Published.}

A HANDBOOK OF THE BROMELIACEAE. Demy 8vo. $5 s$.

LoNdox : GEORGE BELL \& SONS, York Street, Covent Garden 


\section{Recently Completed, with 1937 Full-page Coloured Plates. SOWERBY'S ENGLISH BOTANY.}

Containing a Description and Life-sized Coloured Drawing of every British Plant.

Edited and brought up to the Present Standard of Scientific Knowledge

By the late J. T. BOSWELL, LL.D., F.L.S., \&c.

THE FIgURES BY J. E. SOWERBY, J. W. SALTER, A.L.S., AND N. E. BROWN.

Third Edition, in 12 vols., super-royal 8vo, £24 3s. in cloth, £26 I1s. in half-morocco, and $\$ 30$ 9s. whole morocco. Also \$9 parts, 5s. each, except the Index Part, 7s. $6 d$.

* * The Work has for nearly a century been the chief authority on English Botany, and in its present form may fairly be taken as the most complete representative of the present state of knowledge of British Plants.

\section{CONTENTS.}

VoL. I. Ranunculacer, Berberidaceæ, Nymphæaceæ, Papaveraceæ, and Cruciferæe. Cloth, £I I 8s.; half-morocco, $£_{2}^{2 s}$. ; morocco, £2 8s. $6 d$.

VoL. II. Resedaceæ, Cistaceæ, Violaceæ, Droseraceæ, Polygalaceæ, Frankeniaceæ, Caryophyllaceæ, Portulacaceæ, Tamariscaceæ, Elatinaceæ, Hypericaceæ, Malvaceæ, Tiliaceæ, Linaceæ, Geraniaceæ, Ilicineæ, Celastraceæ, Rhamnaceæ, Sapindaceæ. Cloth, £I I8s. ; half-morocco, $£_{2}^{2} 2 s$. ; morocco, $£_{2} 8 s^{\prime}{ }^{\prime} 6 d$.

VoL. III. Leguminiferæ and Rosacr. Cloth, £2 3s.; half-morocco, £2 75.; morocco, $£^{2} 13^{s} .6 d$.

Vol. IV. Lythraceæ, Onagraceæ, Cucurbitaceæ, Grossulariaceæ, Crassulaceæ, Saxifragacer, Umbelliferæ, Araliaceæ, Cornaceæ, Loranthaceæ, Caprifoliacer, Rubiacex, Valerianaceæ, and Dipsaceæ. Cloth, $£_{2} 8 s$. ; half-morocco, $£^{2}$ I2s.; morocco, $£ 2$ I 8 s. $6 d$.

VoL. V. Compositæ. Cloth, $£_{2} 3$ s. ; half-morocco, $£_{2} 7 s . ;$ morocco, $£_{2}{ }_{13} 3.6 d$.

Vol. VI. Campanulaceæ, Ericaceæ, Jasminaceæ, Apocynaceæ, Gentianaceæ. Polemoniaceæ, Convolvulaceæ, Solanaceæ, Scrophulariaceæ, Orobanchaceæ, and Verbenacex. Cloth, $£$ I I $8 s$. ; half-morocco, $£_{2} 2 s$.; morocco, $£_{2} 8 s .6 d$.

Vol. VII. Labiatæ, Boraginaceæ, Lentibulariaceæ, Primulaceæ, Plunaginacex, Plantaginacex, Paronychiacex, and Amarantacex. Cloth, $\mathscr{E}_{\mathrm{I}} \mathbf{1 8 s}$.; half-morocco, $£_{2} 2 s$.; morocco, £2 8s. $6 d$.

Vol. VIII. Chenopodiacx, Polygonacer, Eleagnacex. Thymela_ex, Santalacere, Aristolochiaceæ, Empetraceæ, Euphorbiaceæ, Callitrichaceæ, Ceratophyllaceæ, Urticaceæ, Amentiferæ, and Coniferæ. Cloth, $£_{2} 13^{s}$.; half-morocco, $£_{2} 17$ s. ; morocco, £3 3s. 6d.

Vol. IX. Typhaceæ, Araceæ, Lemnaceæ, Naiadaceæ, Alismaceæ, Hydrocharidaceæ, Orchidaceæ, Iridaceæ, Amaryllidaceæ, Dioscureaceæ, and Liliaceæ. Cloth, £I I8s. ; half-morocco, $£ 22 s$.; morocco, $£ 2$ 8s. $6 d$.

Vol. X. Juncaceæ and Cyperacex. Cloth, $£_{1} 18 s$. ; half-morocco, $£_{2} 2 s . ;$ morocco, $£ 28$ s. 6 d.

VoL. XI. Graminaceæ. Cloth, £I 13s.; half-morocco, ŁI I7s.; morocco, £2 3s. $6 d$.

VoL. XII. Marsiliacex, Isoetacex, Selaginellacex, Lycopodiacex, Ophioglossaceæ, Filices, Equisetacex, and Characex; and an Index to the whole Work. Cloth, $£$ I 15 s.; half-morocco, $£_{1} 19 s . ;$ morocco, $£_{2} 5$ s. $6 d$. 


UC SOUTHERN REGIONAL LIBRARY FACIUTYY

A $000047152 \quad 4$ 
